\title{
The Constructs of the Living Labs Innovation Platform
}

\author{
by
}

Christ Habib

A thesis submitted to the Faculty of Graduate and Postdoctoral Affairs in partial fulfillment of the requirements for the degree of

$$
\text { Master of Applied Science }
$$

in

Technology Innovation Management

Carleton University

Ottawa, Ontario

(C) 2015, Christ Habib 


\begin{abstract}
Despite the growing popularity of using living labs as an innovation platform, little is known about their characteristics. This research uses a case study approach with content analysis to identify a list of key attributes that help define the concept of living labs. Drawing upon the literatures on user innovation, co-creation, and living labs, theoretical constructs were used to guide the investigation of living lab applications that are publically available through the European Network of Living Labs (ENoLL) website.
\end{abstract}

The results from the research produced nine constructs that give insight into living labs. These constructs reflect both qualified pre-existing notions and disqualified notions of living labs, such as the objective, the degree to which users are involved, and the legal structure for operations. They bring about significant new knowledge regarding the nature of living labs, including their modes of communication, their purposes, handling of intellectual property, and generation of funds. 


\section{Acknowledgements}

I would like to thank all TIM professors, especially my thesis supervisor, Dr. Mika Westerlund: your constant support, encouragements and motivations are very inspiring and I am very grateful to have had the opportunity to learn from and work with you.

I would like to thank my fellow colleagues who have shared their knowledge and experience, personifying the collaborative culture of living labs and edifying me as an individual.

To my family, especially my loving parents: you encouraged me to persevere, inspired me to pursue my dreams and supported me throughout my educational journey. I am forever grateful and appreciative of everything you have ever done for me. I love you.

Lastly, to my friends: the moments we shared, the adventures we pursued and the memories we made will make these past years forever unforgettable. I look forward to the future we have together and the new memories we will forge. 


\section{Table of Contents}

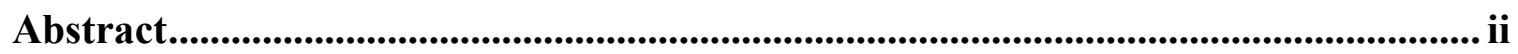

Acknowledgements ......................................................................................................ii

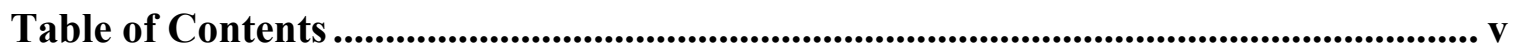

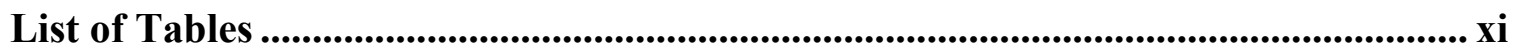

List of Figures............................................................................................................................... xii

List of Appendices...................................................................................................................... xiv

1 Chapter: Introduction .......................................................................................................... 1

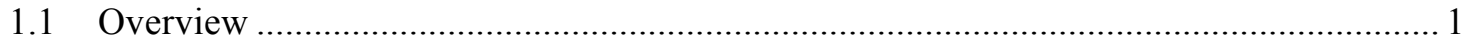

1.2 Objectives and Deliverables …………………………………………………….... 11

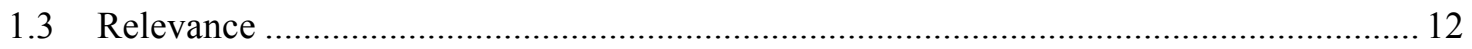

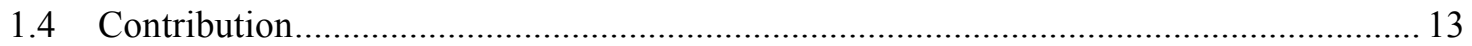

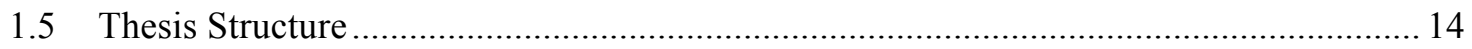

2 Chapter: Literature Review........................................................................................... 16

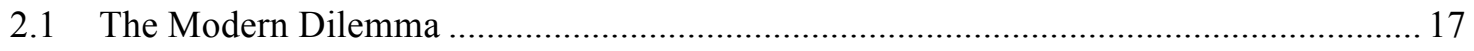

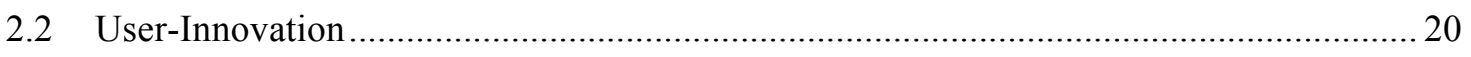

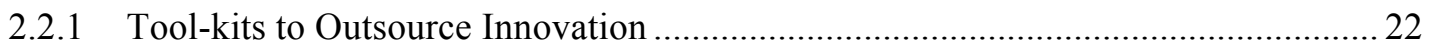

2.2.2 User Communities: Source of Innovation via Tool Kits........................................ 24

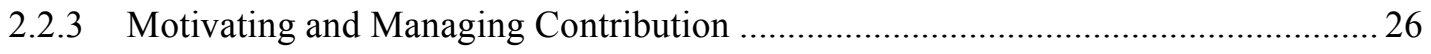

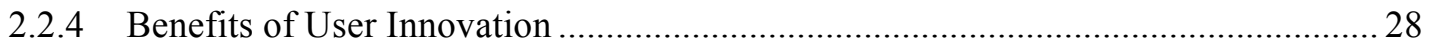

2.2.5 Business Appropriation of Innovation User Communities ...................................... 29

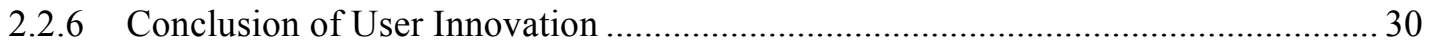

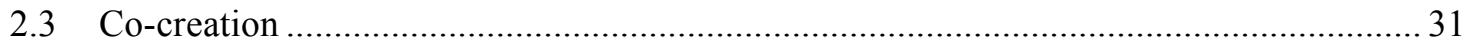

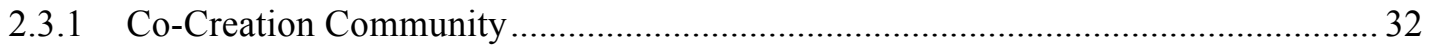




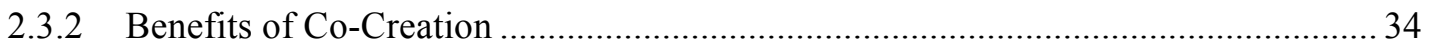

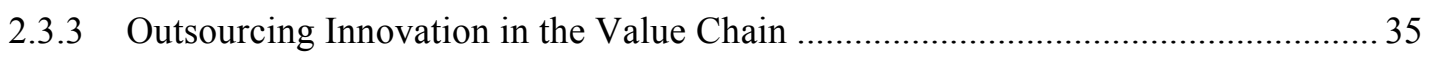

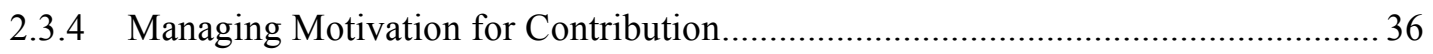

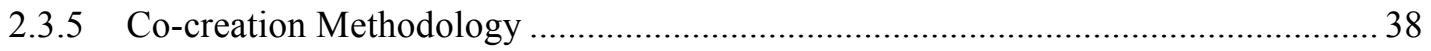

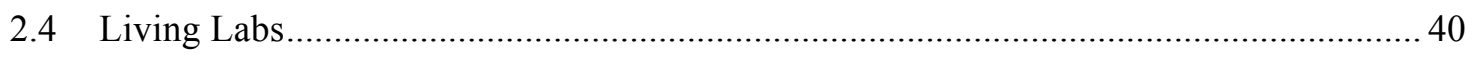

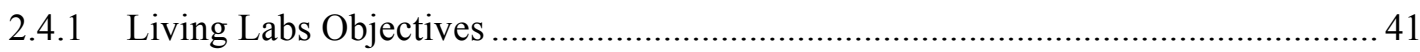

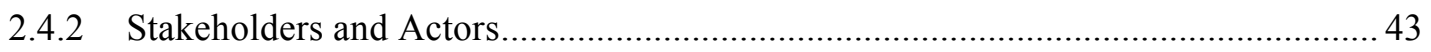

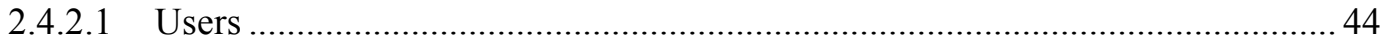

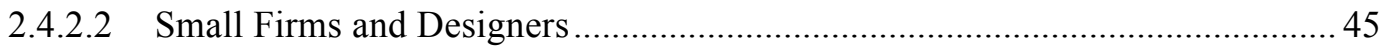

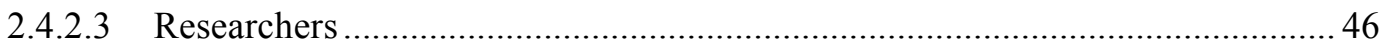

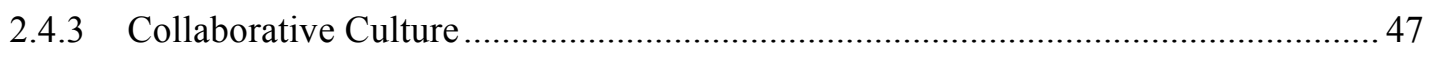

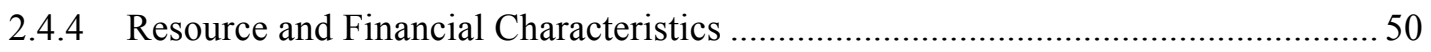

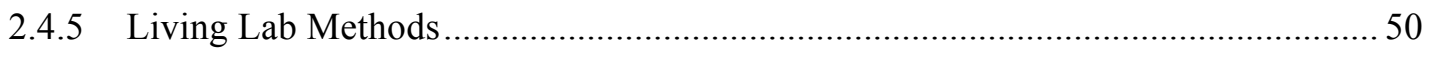

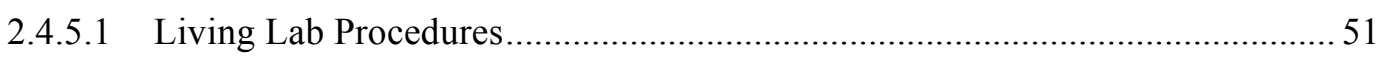

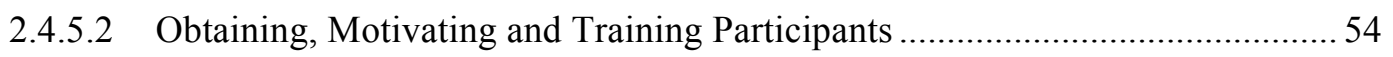

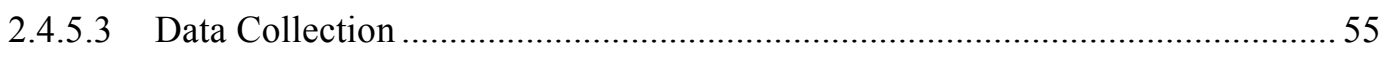

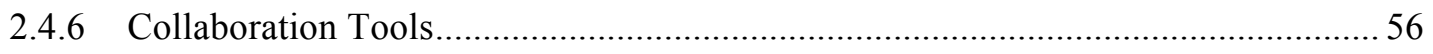

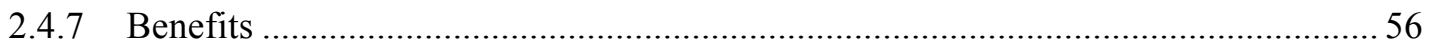

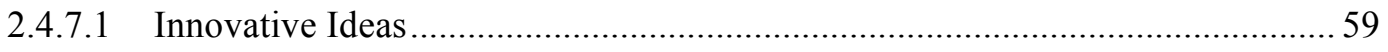

2.4.7.2 Networking Opportunities and Framework ….............................................5 59

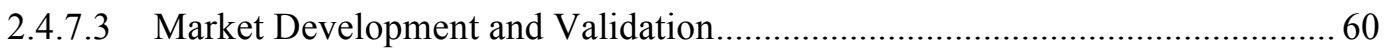

2.4.7.4 Financial Mitigation and Access to Resources .............................................. 61

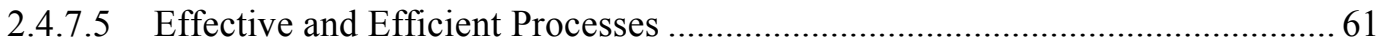

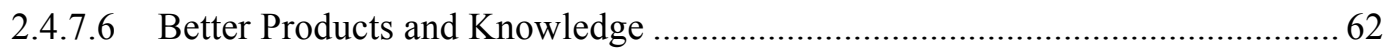

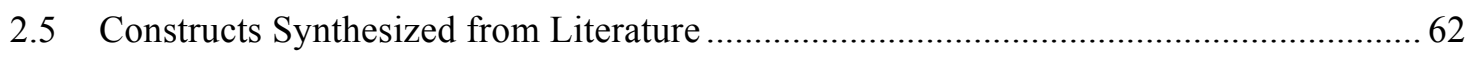

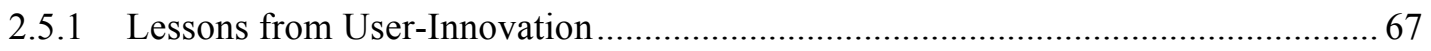




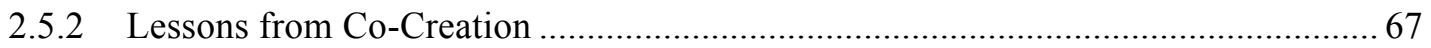

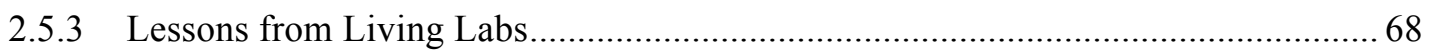

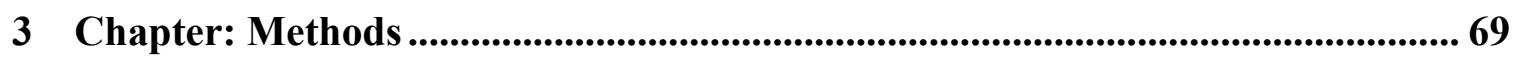

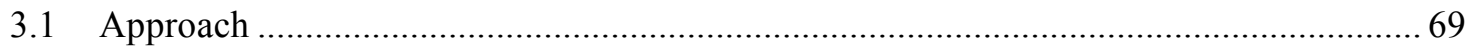

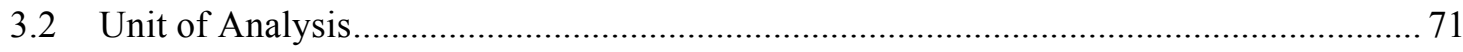

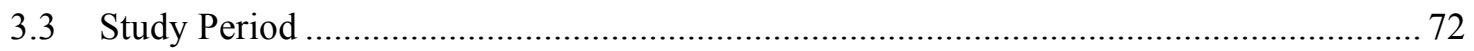

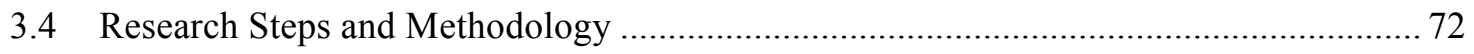

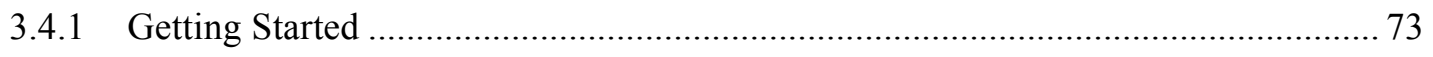

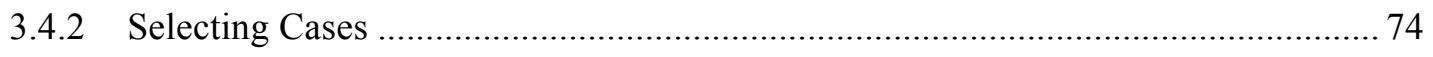

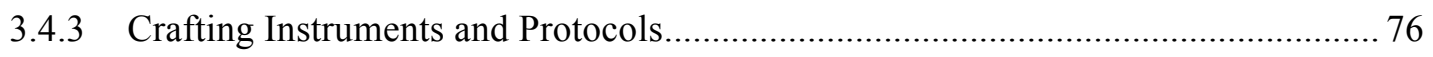

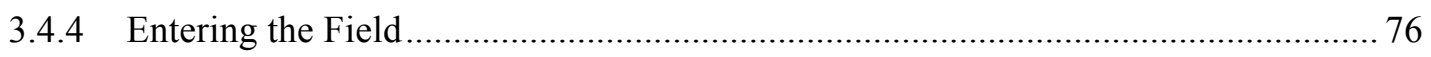

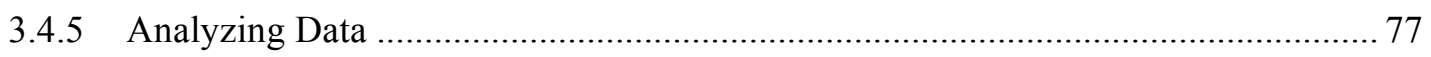

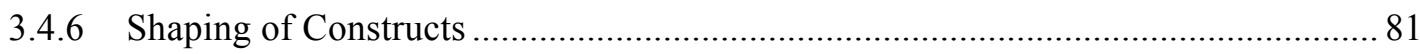

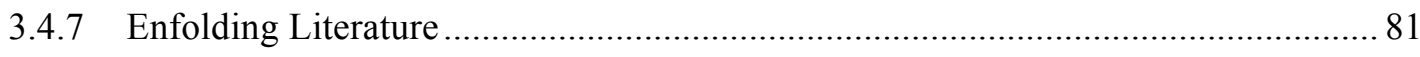

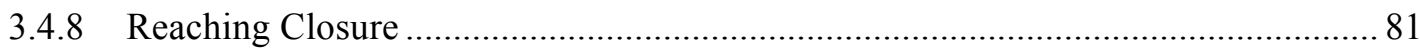

4 Chapter: Methods ................................................................................................ 83

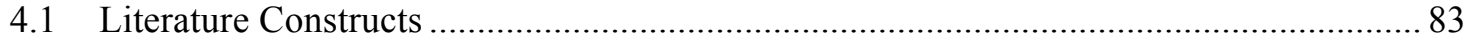

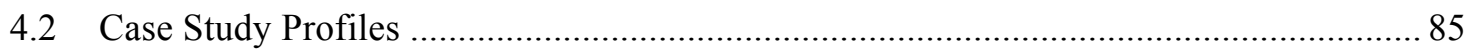

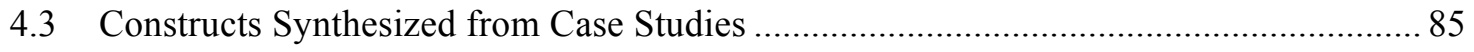

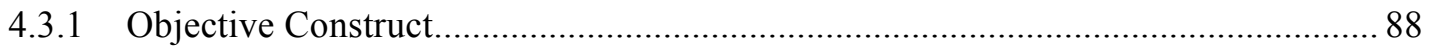

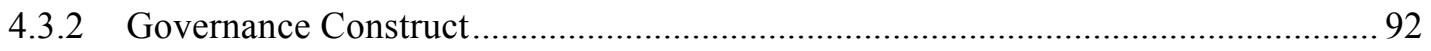

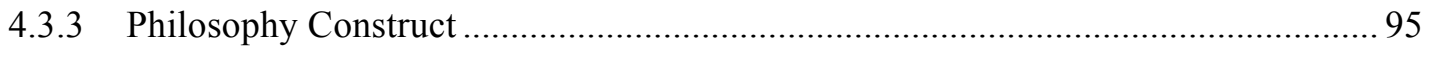

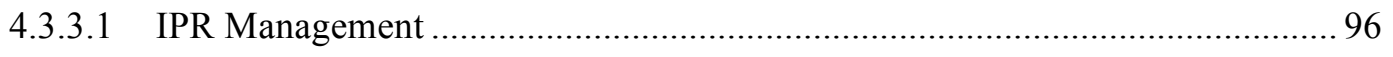

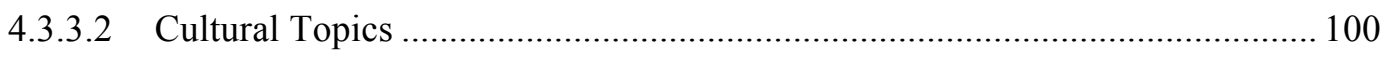

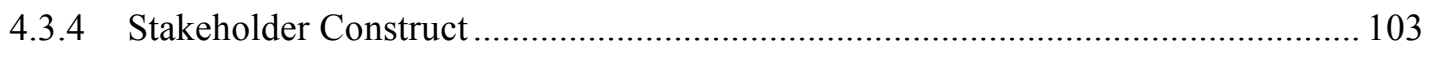

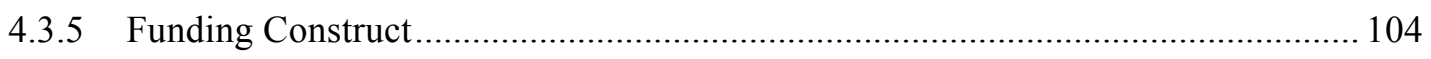




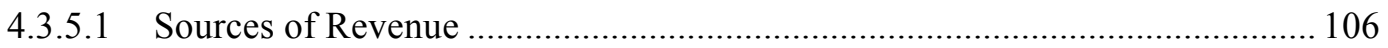

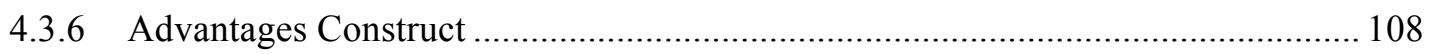

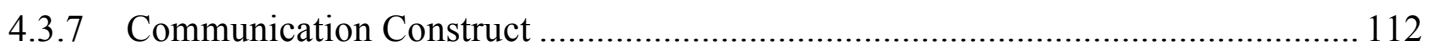

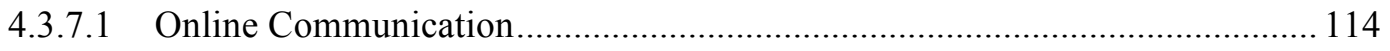

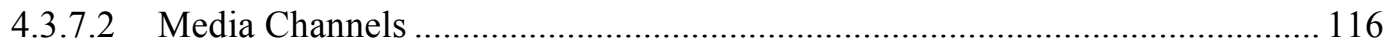

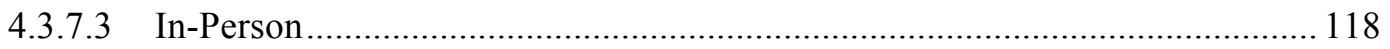

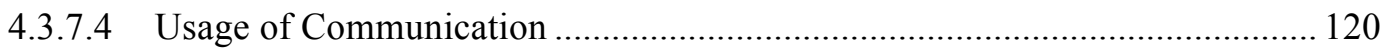

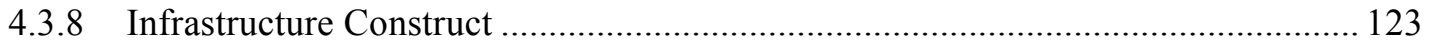

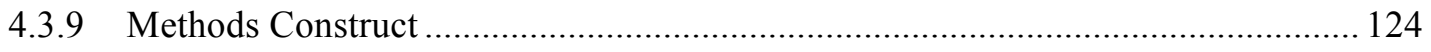

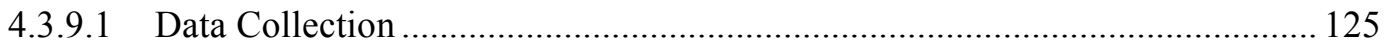

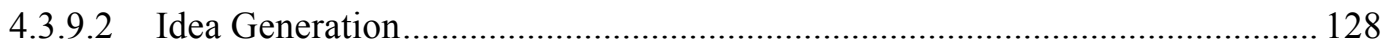

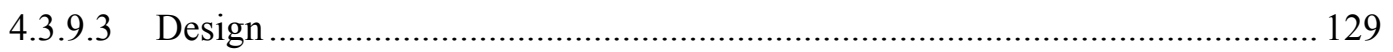

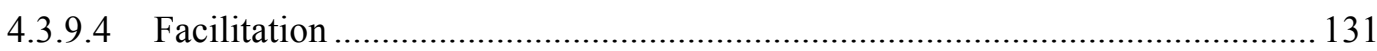

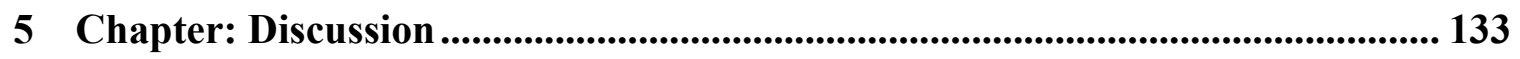

5.1 Results from Case Study Approach and Content Analysis ....................................... 133

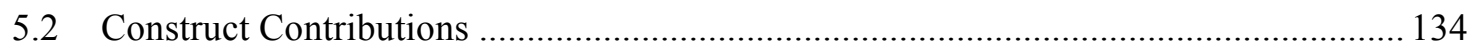

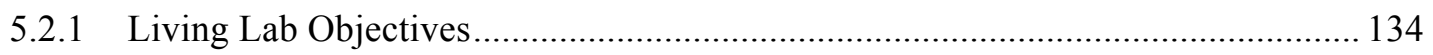

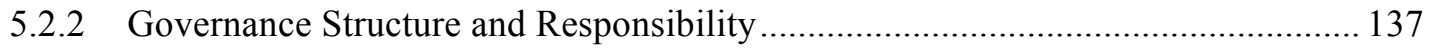

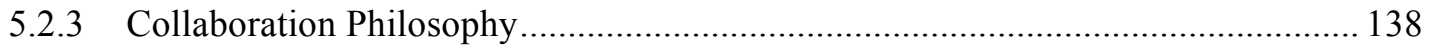

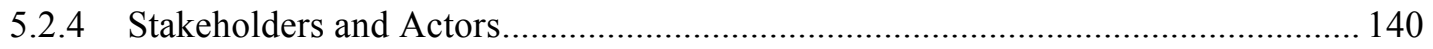

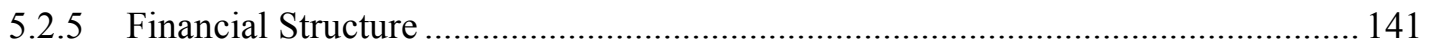

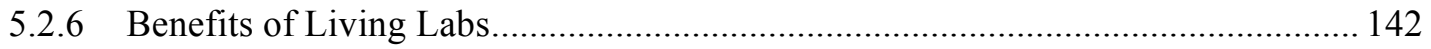

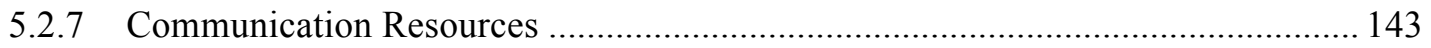

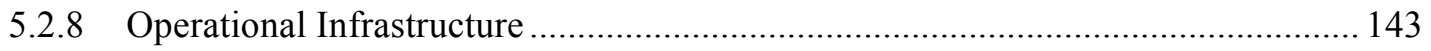

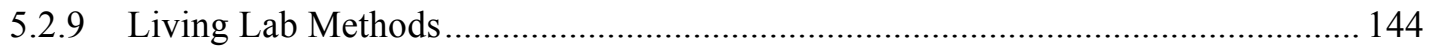




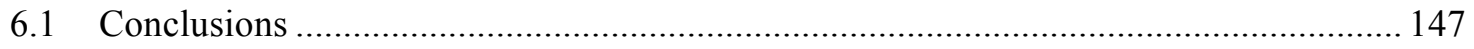

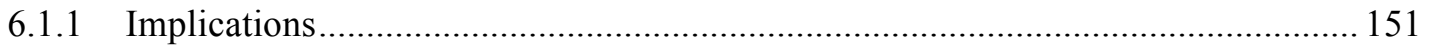

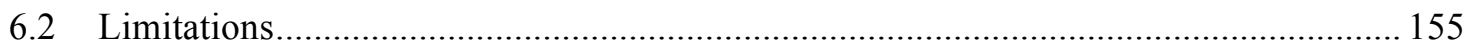

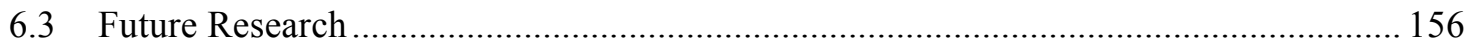

Appendices .......................................................................................................................... 157

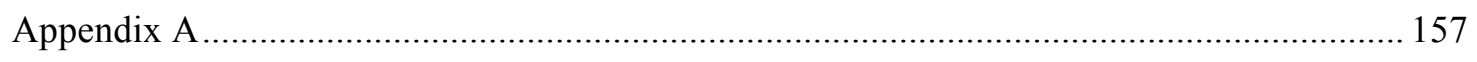

A.1 A Cross-Border Living Lab on Territorial Marketing ......................................... 157

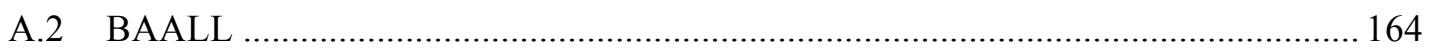

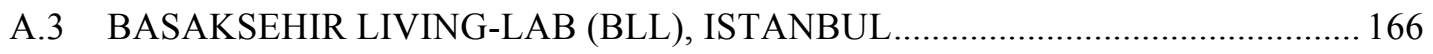

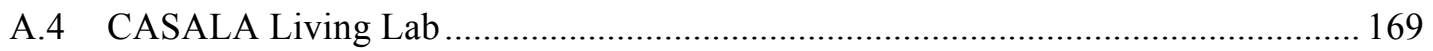

A.5 CIMLAB CaraÄbe Innovation Martinique Living Lab ….................................... 172

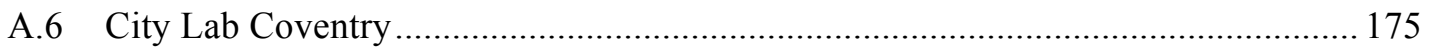

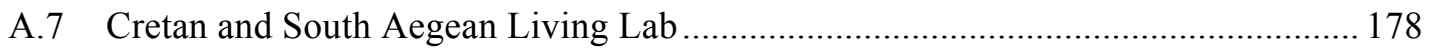

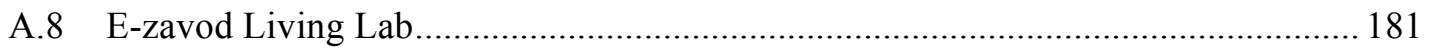

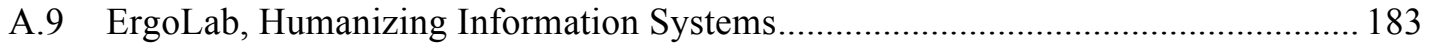

A.10 Espaitec Science and Technology Park Living Lab............................................. 187

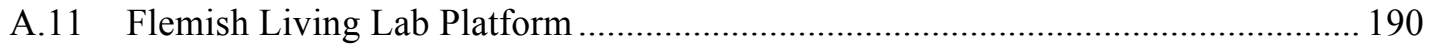

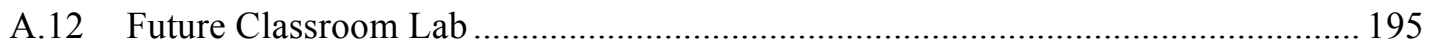

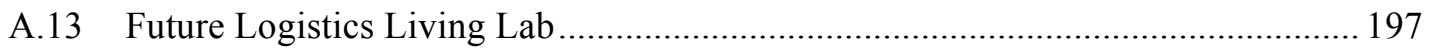

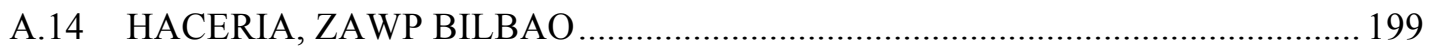

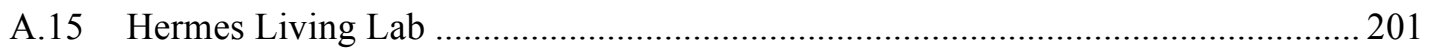

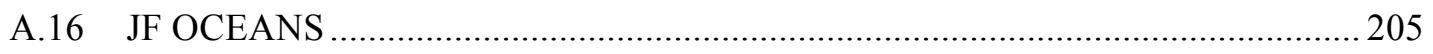

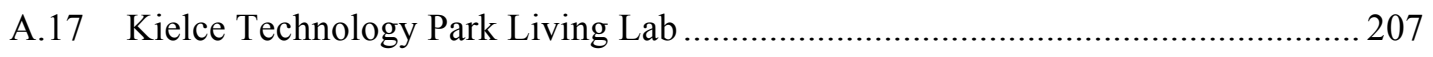

A.18 Knowledge Micro, Small \& Medium Enterprise Living Lab (KMSME LL) ......... 210

A.19 Knowledge Women Innovation Space Living Lab (KWINS LL) ........................ 214 
A.20 LabICT-PA ICT Laboratory for the Public Administration

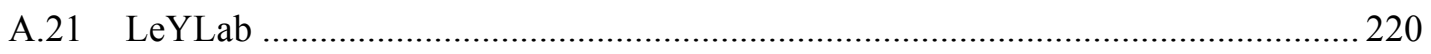

A.22 LIVING LAB CLUSTER TIC BOGOTÁ - COLOMBIA ...................................... 223

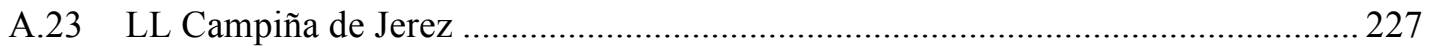

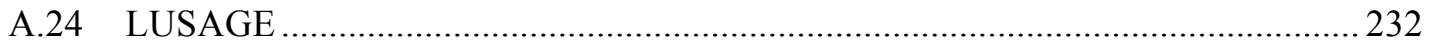

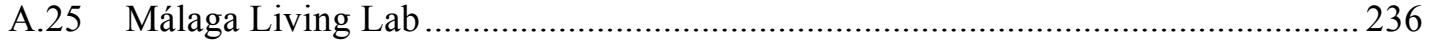

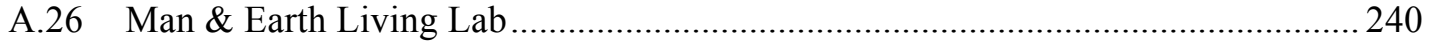

A.27 Middle East Digital Ecosystem Living Lab ...................................................... 244

A.28 MIMMALAB - Interactive Music Museum Living Lab.......................................248

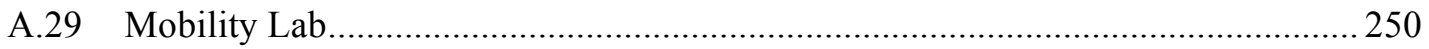

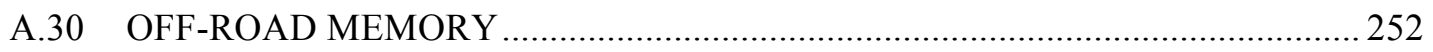

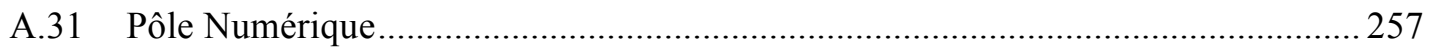

A.32 Regional strategic impact through open innovation and creative use of ICT (LLMidt) 261

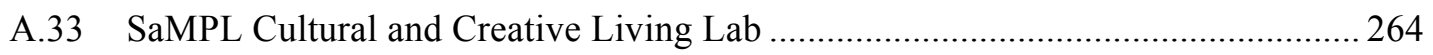

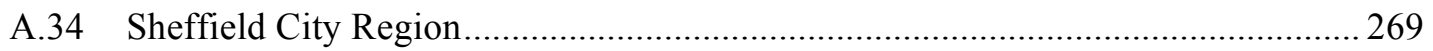

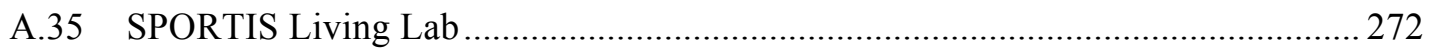

A.36 Telecommunication Networks and integrated Services Living Lab ...................... 276

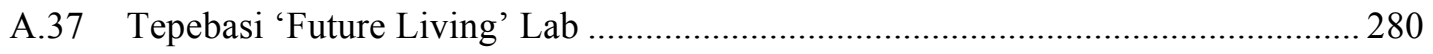

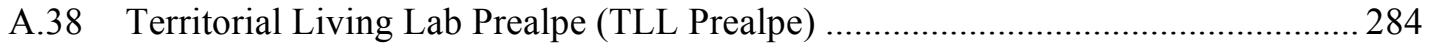

A.39 Urban Living Lab - Versailles Saint-Quentin-en-Yvelines ....................................2 287

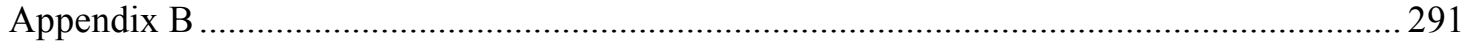

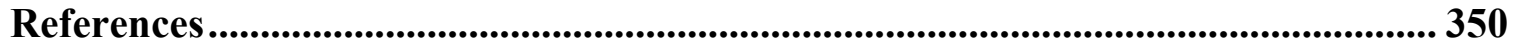




\section{List of Tables}

Table 1 Identified Research Streams of Living Labs (Westerlund \& Leminen, 2014) ... 5

Table 2 Win-Win strategies for living Labs (Guzman, 2013) .................................. 57

Table 3 Emergent constructs comparing innovation initiatives.................................. 63

Table 4 Step-by-step methods with undertaken activity ...................................... 72

Table 5 Questions to Answer during Within-Case Analysis ..................................... 84

Table 6 Emergent Constructs from Living Lab Cases............................................. 85

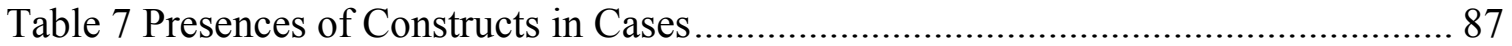

Table 8 Questions to guide establishing a Living Lab............................................. 153

Table 9 Objective Data points Assorted into Respective Categories .......................... 291

Table 10 IPR Management Data points Assorted into Respective Categories............... 310

Table 11 Innovation Culture Data points Assorted into Respective Categories............ 315

Table 12 Revenue and Purpose Data points Assorted into Respective Categories ........ 318

Table 13 Advantages Data points Assorted into Respective Categories ....................... 322

Table 14 Reasons of Communication Data points Assorted into Respective Categories335

Table 15 Methods Data points Assorted into Respective Categories ............................ 339

Table 16 Attracting Users Data points Assorted into Respective Categories................ 346

Table 17 Data Collection Data points Assorted into Respective Categories................. 346

Table 18 Idea generation Data points Assorted into Respective Categories ................. 348 


\section{List of Figures}

Figure 1 Observed and Measured Objectives of Living labs........................................ 89

Figure 2 Observed and Measured Legal Structures of Living labs ................................ 93

Figure 3 Observed and Measured Philosophy Categories for Living labs ..................... 95

Figure 4 Observed and Measured IPR Management Techniques used by Living Labs. 97

Figure 5 Observed and Measured Innovation Culture Topics ....................................... 101

Figure 6 Observed and Measured Sources of Financial Support................................... 105

Figure 7 Observed and Measured Streams of Revenue forming their Business Model 106

Figure 8 Observed and Measured Advantages Living Labs Provides to its Members. 109

Figure 9 Observed and Measured Modes of Communication ....................................... 113

Figure 10 Breakdown of Online Communication Channels Used by Living Labs ...... 115

Figure 11 Breakdown of the Various Medial Channels Used by Living Labs............. 117

Figure 12 Breakdown of the In-person communication channels .............................. 119

Figure 13 Observed and Measured Uses of Communication Resources....................... 121

Figure 14 Observed and Measured topics relating to Living Lab's Innovation Methods 125

Figure 15 Observed and Measured categories of Data Collection Techniques ............ 126

Figure 16 Observed and Measured Sources of Users ……......................................... 127

Figure 17 Observed and Measured Idea Generation Objectives .................................. 128

Figure 18 Observed and Measured Stakeholders who carryout Design Activities ...... 130

Figure 19 Stakeholders of Hermes Living Lab (ENoLL, 2014) .................................. 202

Figure 20 Governance structure of KMSME LL (ENoLL, 2014) ............................... 212 
Figure 21 Governance Structure of KWINS LL (ENoLL, 2014) ............................. 215

Figure 22 Governance Structure of Living Lab Cluster Tic (ENoLL, 2014) .............. 224

Figure 23 Management during Innovation Cycle of Living Lab Cluster (ENoLL, 2014) 227

Figure 24 Stakeholders of SPORTIS (ENoLL, 2014) ......................................... 274

Figure 25 Value Added by Stakeholders of SPROTIS (ENoLL, 2014) ..................... 275 


\section{List of Appendices}

This page lists all of the appendices.

Appendix A 157

A.1 A Cross-Border Living Lab on Territorial Marketing

A.2 BAALL 164

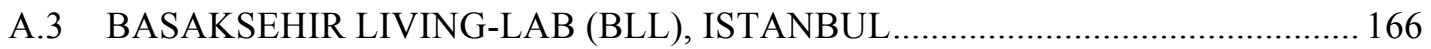

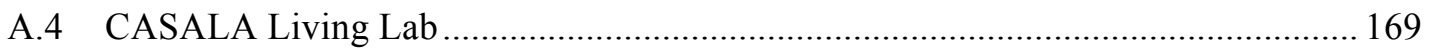

A.5 CIMLAB CaraÄbe Innovation Martinique Living Lab ......................................... 172

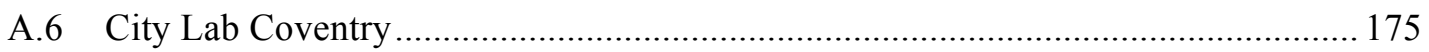

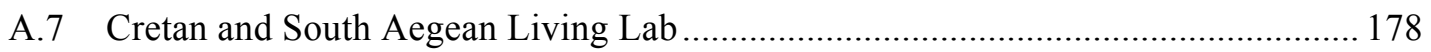

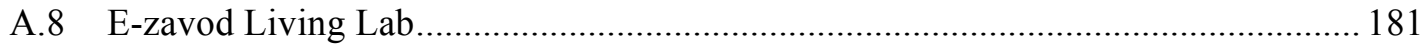

A.9 ErgoLab, Humanizing Information Systems...................................................... 183

A.10 Espaitec Science and Technology Park Living Lab.......................................... 187

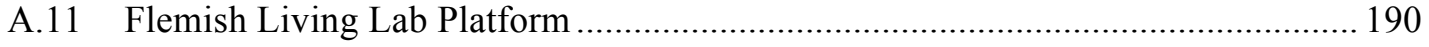

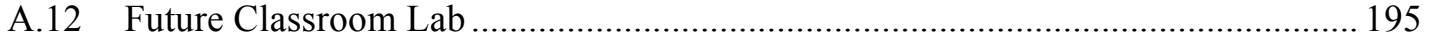

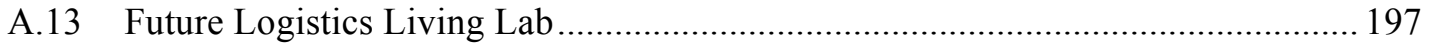

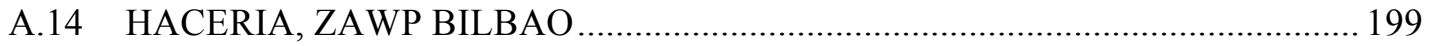

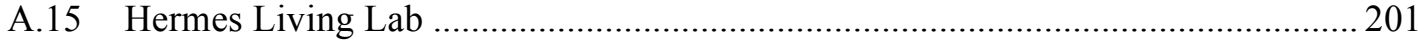

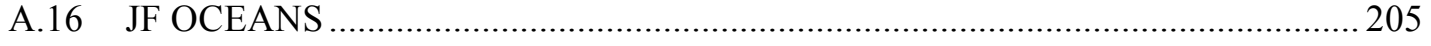

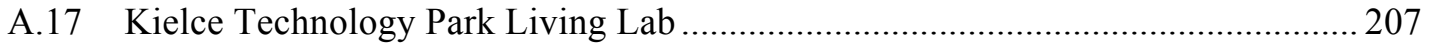

A.18 Knowledge Micro, Small \& Medium Enterprise Living Lab (KMSME LL) ......... 210

A.19 Knowledge Women Innovation Space Living Lab (KWINS LL) ......................... 214

A.20 LabICT-PA ICT Laboratory for the Public Administration ..................................2 217 


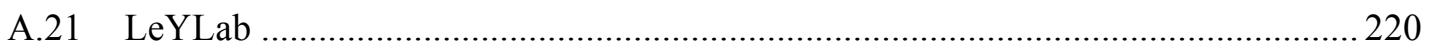

A.22 LIVING LAB CLUSTER TIC BOGOTÁ - COLOMBIA ..................................... 223

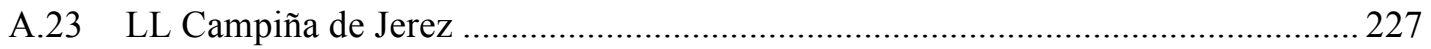

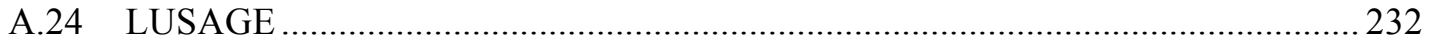

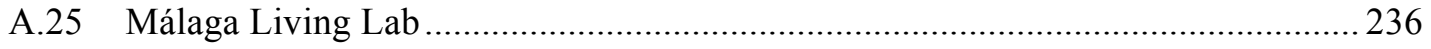

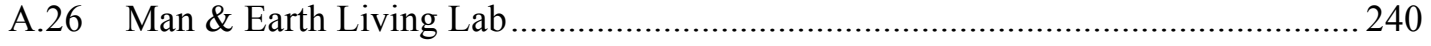

A.27 Middle East Digital Ecosystem Living Lab ....................................................... 244

A.28 MIMMALAB - Interactive Music Museum Living Lab........................................248

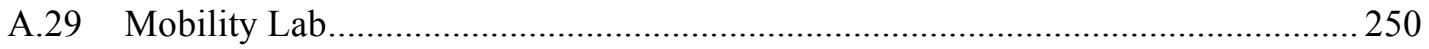

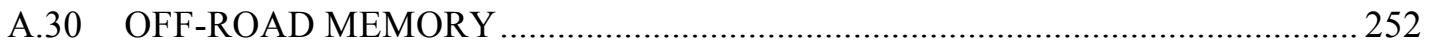

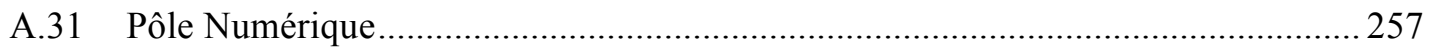

A.32 Regional strategic impact through open innovation and creative use of ICT (LLMidt) 261

A.33 SaMPL Cultural and Creative Living Lab ….................................................... 264

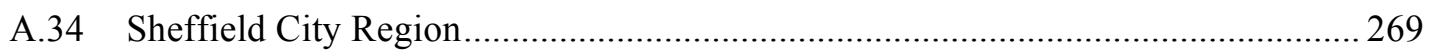

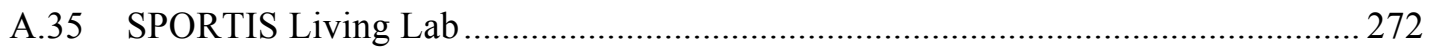

A.36 Telecommunication Networks and integrated Services Living Lab ......................2 276

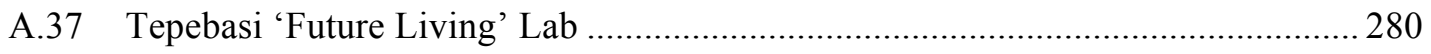

A.38 Territorial Living Lab Prealpe (TLL Prealpe) …................................................. 284

A.39 Urban Living Lab - Versailles Saint-Quentin-en-Yvelines ...................................2 287 


\section{Chapter: Introduction}

This chapter is divided into five sections in order to provide a comprehensive synopsis of the thesis' report. Section 1.1 provides an overview of the thesis research. Section 1.2 describes the objectives of the research and the deliverables it aims to produce. Section 1.3 explains the importance of this research and identifies the groups to whom this research is relevant. Section 1.4 describes the contribution made by this research. Section 1.5 outlines the configuration of this thesis.

\subsection{Overview}

Innovation is no longer an individual's responsibility but rather requires group creativity that is facilitated and supported through interactive processes (Holst, 2007). Living labs concept does this by gathering many stakeholders together to work on the creation, validation, and test of new services, business ideas, markets and technologies in real-lifecontexts. As such, the living lab concept has become increasingly popular over the last ten years. Knight first coined the concept and term "living laboratory" in 1749 (ref. Westerlund \& Lemunen, 2014) where it was first referred to as an environment of an experiment. The modern concept roots of back to Professor William Mitchell of MIT, Boston, in which he setup a real home environment for the purpose of investigating the application of smart home systems in the day-to-day activities of humans (Eriksson et al., 2005). His living lab represented the first user-centered research approach for sensing, 
prototyping, refining, and validating the introduced technologies within the context of the ever-chaotic real-life scenarios (ENoLL, 2010).

Since its conception, the idea of living labs has evolved and sprouted into many fields of research. Domains of living labs vary drastically and can include smart-cities, egovernance, e-health, security, sustainable energy, logistics, internet of things, media and much more (ENoLL, 2010). According to Almirall and Wareham (2008), the common notion of all living labs is bringing together several stakeholders including users, on equal terms for the purposes of co-creating innovations through experimentation in real-world settings. The lab itself provides the structure and governance for the collaboration. Typical living labs will undertake projects and engage companies, academia, government/public, associations and users to iteratively develop and validate the innovations (Almirall \& Wareham, 2011).

Living lab concept is unique in that it harmonizes society, technology and markets together in the real-life scenarios; it targets a specific industry (market), introduces a new product (technology) and tests it in a day-to-day activity that includes other products, people and problems (society). The business application of this concept can be explained through three statements that clarify the outcome of innovation risk based on influencing factors (Eriksson et al., 2005):

1) If only society and technology are involved, the results could be un-economical

2) If only society and market are involved the result could be old-fashioned 
3) If only market and technology are involved the result could have low acceptance

The living lab concept, mostly used by high-tech firms, has now become a new way of harnessing user innovation with the added benefit of validation (Guzman et al., 2013). For this reason living labs have experienced a drastic growth in the past decade. With close to 400 labs now in operation around the world, and a majority located in Europe, the European Network of Living Labs (ENoLL) was formed to help bridge the labs for the sharing of knowledge, methodologies and best practices.

The knowledge generated about living labs to date has been diverse in nature. Researchers have studied the living labs concept through different lenses (cf. Westerlund \& Leminen, 2014) and examined a wide range of topics including innovation methodologies, information flow structures, innovation outcomes, community operations, methods, and frameworks. Because living labs have been associated with complex innovation concepts that are multidisciplinary (Fulgencio et al., 2012), studies in the field have become scattered setting assumptions to explain the phenomenon (Westerlund \& Leminen, 2014). Leminen (2013) argues that the lack of a proper definition for living labs is the cause of such disconnected research.

Some researchers have chosen to define living labs as a "user-driven innovation infrastructure" for designing and validating products, because of its unique use of test environments for real-life extermination (Kareborn et al., 2010). This definition defaults 
the living lab concept to a physical or virtual (Guzman et al., 2013) laboratory where analysis on technical and socio-economic attributes could be conducted for the purposes of solving people's needs.

Erikkson and colleagues (2005) define living lab as "user-centric research methodology" because it has a framework and process of sensing, prototyping, validating and refining innovations using real world environments. According to ENoLL (2007), some labs have defined themselves as "both a methodology for user driven innovation and the organization that primarily use it".

Ballon and colleagues (2005) describe living labs as a test and experimentation platform that addresses key problems when bringing technology to market. Pierson and Lievens (2005) also refer to it as a 'test and experiment action platform'. They explain that living labs is an infrastructure based on a methodological innovation framework that combines users and researchers together to design, develop and evaluate innovations in the early stages of a products life cycle. When firms test their products in real-life scenarios, it is often done with a product that has received significant investment and is near maturity. Comparatively, living labs focuses on experimentation across the full cycle of research, design and testing of products. Mulder (2012) calls it a refining process that begins by sensing, then prototyping and then validating solution in an evolving real life context.

Corelabs (2008) defines and describes the concept as an approach with "functional regions where stakeholders have formed a Public-Private-Partnership ... all collaborating 
for creation, prototyping, validating and testing new services, products and systems". As explained by Sleeswijk and colleagues (2005), co-creation process involves more than a methodology. While participants (ex. Users) bring their expertise and experiences, it is important for designers and researchers to offer support by providing tools and resources (Sanders \& Stappers, 2008). Living labs therefore could be viewed as structures or societal resources that set frameworks, experiences, and conditions to develop ideas into innovations (VINNOVA, 2009).

However, to suggest that living labs only function at the level of research and development and not actual exploitation, still lead to inaccurate conclusions of the concept (Almirall and Wareham, 2011). According to Liedtke and colleagues (2012), living labs are an innovation approach that optimizes both the production and consumption of products and services.

Westerlund and Leminen (2014) attempted to compile the various definitions used to describe living labs by examining the many research streams as seen in table 1 . As seen in column one, the research avenue captures the definition of living lab that guided the study while the characteristic column tries to capture the essential elements.

Table 1 Identified Research Streams of Living Labs (Westerlund \& Leminen, 2014)

\begin{tabular}{|l|l|l|}
\hline \multicolumn{1}{|c|}{ Research Avenues } & \multicolumn{1}{|c|}{ Characteristics } & \multicolumn{1}{c|}{ Examples } \\
\hline Living lab as a system, an & Living lab is a system of & Regional system \\
ecosystem or a network & connected links, an & \\
\hline
\end{tabular}




\begin{tabular}{|l|l|l|}
\hline Research Avenues & \multicolumn{1}{|c|}{ Characteristics } & \multicolumn{1}{|c|}{ Examples } \\
\hline & $\begin{array}{l}\text { ecosystem or a network of } \\
\text { diverse actors, activities, } \\
\text { and resources. }\end{array}$ & Oliveira et al., 2006 \\
& Innovation system \\
& Ballon et al. 2005; Eriksson \\
et al., 2005 \\
Ecosystem
\end{tabular}




\begin{tabular}{|c|c|c|}
\hline Research Avenues & Characteristics & Examples \\
\hline approach & $\begin{array}{l}\text { various design and research } \\
\text { approaches and their } \\
\text { combinations. }\end{array}$ & \\
\hline $\begin{array}{l}\text { Living lab as an } \\
\text { environment with } \\
\text { embedded technologies and } \\
\text { users }\end{array}$ & $\begin{array}{l}\text { Living labs is a real-life } \\
\text { environment to demonstrate } \\
\text { goals and learn with users } \\
\text { with the help embedded } \\
\text { technology. }\end{array}$ & $\begin{array}{l}\text { Intille et al. 2005; Intille et } \\
\text { al. 2006; Bajgier et al., } 1991\end{array}$ \\
\hline $\begin{array}{l}\text { Living lab as a context } \\
\text { and/or methodology }\end{array}$ & $\begin{array}{l}\text { Living lab provides a } \\
\text { context, milieu and/or } \\
\text { methodology. }\end{array}$ & $\begin{array}{l}\text { Bergvall-Kåreborn et al., } \\
\text { 2009; Mulder and Stapper, } \\
\text { 2009; Almirall et al., } 2012\end{array}$ \\
\hline $\begin{array}{l}\text { Living lab as a tool for the } \\
\text { enhancement and } \\
\text { implementation of public } \\
\text { and user involvement }\end{array}$ & $\begin{array}{l}\text { Living lab offers a } \\
\text { mechanism for enhancing } \\
\text { and implementing public } \\
\text { and user involvement; there } \\
\text { are multiple views on how } \\
\text { to categorize these }\end{array}$ & $\begin{array}{l}\text { Rural innovations } \\
\text { Schaffers \& Kulkki, 2007; } \\
\text { Mutanga et al., } 2011 \\
\text { Regional innovations } \\
\text { Rasanen; Juujärvi \& Pesso, }\end{array}$ \\
\hline
\end{tabular}




\begin{tabular}{|c|c|c|}
\hline Research Avenues & Characteristics & Examples \\
\hline & mechanisms. & $\begin{array}{l}2013 \\
\text { Smart Cities } \\
\text { Ballon et al., 2011; } \\
\text { Enabler driven or user } \\
\text { driven innovations } \\
\text { Leminen \& Westerlund, } \\
\text { 2012; Leminen et al., 2012; } \\
\text { Leminen, 2013, Leminen et } \\
\text { al., 2014b } \\
\text { PPP, Public-Private } \\
\text { Partnership Niitamo et al., } \\
\text { 2006; Lepik et al., 2010 } \\
\text { Quadruple Helix, a public } \\
\text { private people partnership } \\
\text { (4P) } \\
\text { et al., 2011; Molinari, } 2011\end{array}$ \\
\hline
\end{tabular}




\begin{tabular}{|c|c|c|}
\hline Research Avenues & Characteristics & Examples \\
\hline $\begin{array}{l}\text { Living labs as a } \\
\text { development project for } \\
\text { products, services and } \\
\text { systems }\end{array}$ & $\begin{array}{l}\text { Living lab is a temporary } \\
\text { real-world project for the } \\
\text { purposes of learning, } \\
\text { development, information } \\
\text { provision, prototype testing, } \\
\text { or a mechanism for } \\
\text { implementing provisional } \\
\text { public involvement. }\end{array}$ & $\begin{array}{l}\text { Bajgier et al., 1991; Lasher } \\
\text { et al., 1991; Bengtson, } 1994\end{array}$ \\
\hline $\begin{array}{l}\text { Living lab as a business } \\
\text { activity or operational mode }\end{array}$ & $\begin{array}{l}\text { Living lab is described as } \\
\text { an ongoing business activity } \\
\text { mostly from a process point } \\
\text { of view or considered as an } \\
\text { operational mode. }\end{array}$ & $\begin{array}{l}\text { Schuurman et al., 2012, } \\
\text { Schuurman et al., 2013; } \\
\text { Veeckman et al., } 2013\end{array}$ \\
\hline $\begin{array}{l}\text { Living labs as an innovation } \\
\text { management tool }\end{array}$ & $\begin{array}{l}\text { Living lab is a method for } \\
\text { managing innovation in } \\
\text { ecosystems and/or practical } \\
\text { tool for organizations to } \\
\text { create and manage their } \\
\text { user-based innovation }\end{array}$ & Edvarsson et al., 2012 \\
\hline
\end{tabular}




\begin{tabular}{|l|l|l|}
\hline Research Avenues & \multicolumn{1}{|c|}{ Characteristics } & Examples \\
\hline & processes & \\
& & \\
\hline
\end{tabular}

Unfortunately, as it can be seen, both literature and the current existing living labs do not provide or agree on a standardized concept of living labs or settle on one unique definition. Researchers cannot only agree on a definition of living labs (cf. Nystrom et al., 2014) but also cannot specify the constructs that characterize the structure or operations of living labs (cf. Dell'Era \& Landoni, 2014). Moreover, the resulting research from the various definitions has attempted to consolidate the innovation practices of living labs into limited perspectives and thus possibly misrepresented actual construct of the concept.

Leminen (2013) argues that clear definition needs to be settled and constructs describing the phenomenon need to be developed. From a subjective observation, it appears that the proposed definitions are formulated around attributes that make this new innovation concept unique. Such attributes can be identified as test environment, methodology, structure and outcome. It is therefore the intent of this research to further the scholarly knowledge of living labs by establishing the various attributes of living labs. A collection of attributes can help better define living labs and set various streams to direct future research on the topic of living labs. 
In this thesis, a review of the existing research is done and compared with other innovation research such as user innovation and co-creation to develop a roadmap by which we can examine existing living labs cases and determine their defining attributes. We use a case study approach as set out by Eisenhardt (1989) to understand living labs on case-by-case level and to formulate a more generalized set through a cross-case analysis.

\subsection{Objectives and Deliverables}

The objective of this research is to identify the constructs of living labs, which are the inherent/fundamental characteristics and/or parts of the phenomenon and to identify the popular themes of each construct. These constructs will not only help establish a more robust definition, but also aid in guiding future research.

While these constructs may include preexisting research areas of living labs such as methodology or governance, it is not the aim of the study to generate, test or validate frameworks or models regarding living lab processes or structures. For the purposes of this study, no previously proposed definition of living lab shall be used to narrow the research into a particular stream that has been previously explored.

This research delivers:

1. A Table (3) comparing attributes of living labs against user-innovation and cocreation based on common constructs and knowledge derived from literature. A 
collection of preexisting living lab research will provide an estimation of the innovation constructs and their relation to other innovative concepts.

\section{Figures (in Results section) that visually display the frequency of themes within} each construct of living labs. The examination of existing and operational living labs will form an assortment of case that will outline the internal makeup of living labs and will help describe the constructs of living labs.

3. A list of construct for living labs (Table 6). The list provides focus points for describing the innovation platform for living labs. It will allow for better communication regarding living lab's composition and a baseline for future research.

4. In conclusion, a definition for living labs based on constructs. A single phrase that captures the findings will help simplify the research and give an easier explanation to living labs.

\subsection{Relevance}

Academics and research organizations will find this research relevant because it extends the knowledge on living labs by establishing a set of attributes that can help standardize how living lab concepts are viewed (cf. Mulder, 2012). Practitioners are demanding 
models that include effective practices to guide this kind of shared innovation infrastructure (Guzman et al., 2013). This model sets a starting point for future research.

This research is also relevant to technology entrepreneurs who seek to successfully develop and launch innovative technologies or related services without the risk of market rejection. While much is now known about the value innovative users bring (von Hippel, 2003) and that living labs is the optimal way of producing and commercializing innovation (Liedtke, 2012), there is little guidance as to what is involved in or to how to establish a living lab. Likewise, this research will help users and their associated communities to understand living labs; the model can alleviate the co-operation and trust issues that are required for the successful operations of what is often a bottom-up structure (Barcenilla \& Tijus, 2012; Bonaccorsi \& Rossi, 2003).

\subsection{Contribution}

This research makes a theoretical contribution to the field of innovation and living labs by establishing a definition for living labs and describing the constructs of living labs. It adds a list of nine constructs: objectives, governance, philosophy, stakeholder, funding, advantages, communication, infrastructure and methodology, which help with the understanding of living labs from multiple perspectives. Additionally, this research adds discovered patterns in each construct that give insight into the current operational structure of living labs. 


\subsection{Thesis Structure}

This thesis is organized into six chapters. Chapter 1, the 'Introduction', presents the thesis research outlining the objective of the study, the deliverables, relevance and contribution. Chapter 2, the 'Literature Review', examines the pertinent academic research papers previously conducted in this space by which, the generated lessons learned is used to construct the initial guidelines to conduct the research. Chapter 3, the 'Methods', details the research approach of empirically analyzing the case study data. Chapter 4 , the 'Results', describes the reviewed living labs, their key attributes and the innovation patterns derived from the insights. Chapter 5, the 'Discussion', reviews the research results in comparison to established theory. Chapter 6, the 'Conclusion', identifies the limitations of the research, opportunities for future research and closing thoughts.

\section{Glossary of Terms}


Advantages: The benefits the stakeholders gain from their membership and participation within the living lab.

Coding: Technique used to look for distinct concepts and categories within the data.

Component: An element of the innovative solution.

Communication: The channels, technology and techniques used to network stakeholders for information exchange.

Content Analysis: A method that enables a more objective evaluation of textual data, by counting the recording units versus comparing text based on impressions of a the researcher.

(Emergent) Construct: An idea that enfolds various conceptual elements that is subjectively derived through observed evidence.

Funding: The means by which the living lab financially supports its innovation activities.

Governance: A structural and/or procedural model by which decisions for the innovation projects, process or organization are made.

Infrastructure: The necessary resources and specialized equipment required to carry out the innovation activities.

Methods: The procedural steps used for the inception, development and deployment of innovation.

Objective: The goal to be achieved as determined by the positive impact that the innovation output is expected to produce. 
Philosophy: Mindset of the organization that is reflected in their level of openness and collaboration.

Recording Unit: the measured unit within the content analysis technique. Could be a word, phrase, theme or entire document.

Stakeholder: Entities that add value to the living lab.

Test Environment/Bed: A functional setting (physical or virtual) that aids in the testing cycle of an innovation.

\section{Chapter: Literature Review}


Chapter 2 reviews the literature streams that are relevant to this research. The chapter is organized into five sections. The first section establishes the need for new innovation concepts such as living labs by drawing on the pitfalls of traditional/internal innovation attempts by firms. The next two sections examine briefly the evolution of collaborative innovations i) User-Innovation and ii) Co-Creation. The third section is a collection of what is currently known or postulated about Living Labs. The final section summarizes the lessons learned and synthesizes the initial constructs used to guide the case study research.

\subsection{The Modern Dilemma}

Sharks, commonly referenced for their predatory and dominant attributes, are the perfect metaphor for businesses. Compared to their traditional use of imagery, the reason sharks best represent business is because they must 'keep moving forward' in order to stay alive. Businesses (or equivalently corporation/firms) need to remain innovative and keep moving forward by creating new products and/or services for their consumers.

Firms typically formulate their innovations around their own unique competencies such as their internal technological or scientific knowledge, established human resources, geographical location, and/or strong financial assets (Chesbrough, 2011). These competencies, which typically give the firm a competitive advantage, are purposefully

developed in secret to the outside world in order to maintain their competitive edge and 
first mover advantage in the market (Lee et al., 2012). Unfortunately, the internal development of such competencies tends to create a biased understanding of goods (i.e. products and services) and their associated value and prospects, resulting in their poor innovation by the firm (Eriksson et al., 2005). What new goods such firms do produce risks the possibility of not being demanded whatsoever by the consumer or not appropriately addressing the consumer's need. This problem is highlighted by Bonaccorsi and Rossi (2003) stating that consumers consider commercial software as 'not being very reliable' because they are produced by a limited group of programmers who are bound by market laws.

Franke and von Hippel (2003) add that firms further limit their own innovation by investing in the research and development of separate market segments, keeping the produced goods disjoint. Maintaining a disjoint portfolio of goods would make economic sense as it allows for each segment to have its own technical knowledge, innovation production, market commercialization and assigned resources. However, because humans are complex consumers, each with their own blend of problems and needs, it becomes inefficient to create solutions that are disjoint from each other (Franke \& von Hippel, 2003; Erikkson et al., 2005).

Customers/users end up mixing and matching elements from different market offerings to create a personalized solution (Auh et al., 2007). Through Internet technology and social platforms, consumers are not only producing their own solutions, but are sharing and obtaining what they need from each other (Lee et al., 2012). Firms can capitalize on 
these user innovations because consumers don't often try to gain financial benefits from their own solutions (Bogers \& West, 2012). Regrettably, most firms do not harvest user innovation because they view users as test subjects rather than valued partners (Eriksson et al., 2005); they instead engage in market research to discover the latent needs of the consumer (Saarijarvi, 2012).

It cannot be denied that consumers know their activities, preferences, and needs well while firms have the resources and know-how to produce goods (O'Hern \& Rindfleisch, 2009). However, the innovation cycle is often a trial and error like process with back and forth responses of the consumer trying to convey their needs and the firm attempting to build a solution (Eriksson et al., 2005). The ideal answer is to employ users as the innovation developers and harness the firms' ability of control to organize the innovation efforts effectively (Kogut \& Zander, 1992; Galunic \& Rodan, 1998). Providing bilateral access between consumers and firms, such as the case in Living Labs, is an elegant solution that helps bridge the gap between user innovation and firm capabilities. This solution compensates for poor innovation processes by firms, mitigates the risks associated with market commercialization (Liedtke et al., 2005) and results in sustainable advantages for the firm (Mattson, 2010).

Living labs' open approach allows for firms to collaboratively shape innovation with the consumer (Eriksson et al., 2005). The key feature of living labs is the real-life study of consumers' usage of goods (Mulder, 2012) that gives insight into the consumers' daily interactivity with firms' offerings (Saarijarvi, 2012) giving rise to iterative development 
and market evaluation. Prior qualitative research of living labs (e.g., Pierson \& Lievens, 2005) analyzes the activities of the consumer; especially the uses of their goods in relation to other market offerings that may be disjoint (Liedtke et al, 2012). The activities of living labs further the innovation process by encouraging consumers to socialize, suggest ideas, and engage in innovation development (Russo-Spena \& Mele, 2012).

Since there is currently no proper way to describe the innovation platform for living labs, it will be difficult for firms to develop, operate or incorporate one into their existing innovation processes (Guzman et al., 2013). How customers and other actors engage with the company in collaborative value co-creation activities need to be modeled (Frow et al., 2011). Firms need guidelines to align their innovation processes and resources together with the openness of living labs (Pierson \& Lievens, 2005; DeFillippi \& Roser, 2014). For this reason, this research first explores the fundamentals of user innovation literature and then examines the co-creation literature to understand the collaborative innovation practice between firms and users. Finally, previous literature on living labs is reviewed and analyzed to recognize the evolutionary progression of the innovation process.

\subsection{User-Innovation}

Consumers have very complex needs. As Franke and von Hipple (2003) found, consumers are not fully satisfied with standard product(s) because their needs are heterogeneous. Their research suggests that many commercial products do not serve the 
heterogeneous needs posed by consumers today. Undoubtedly, discovering the heterogeneous needs of users is difficult with conventional market research techniques not to mention a costly activity (von Hippel \& Katz, 2002). This is due to the sticky nature of the consumer needs, which makes it difficult to extract essential information to help build a solution.

In response to the heterogeneous needs of consumers, firms divide consumers into a few market segments and produce goods that will satisfy the average need of the consumer in that segment (Franke \& von Hippel, 2003). As a result, customers will seek custom suppliers to produce a solution that solves their specific (not average) need, or, those with the necessary skills will modify a commercially available product or design from scratch a solution that better addresses their needs. Because the process of producing and commercializing goods is costly, it is logical to only serve a few market segments.

Franke and von Hippel (2003) show that customers are willing to pay premiums for products that satisfy their particular and complex needs. It would therefore be logical for the firm to increase, by differentiation, the number of market segments they serve, invest in a deeper understanding of the user, and capitalize on the premiums while also expanding their innovation portfolio. However, due to the sticky nature of the users' needs, it is better suited for the firm to abandon their attempt at understanding user needs altogether. Instead, firms should outsource the task by equipping users with the necessary resources and permissions to assist them in developing their own custom solution. As reported by Apache users in the study by Franke and von Hippel (2003), the permitted 
ability to modify the original product allowed them to amend libraries for novel usages of the system.

Several stakeholders such as manufacturers, suppliers and research institutions also have sticky information and can output innovation; entities who innovate tend to build upon their sticky information (Bogers \& West, 2012). By providing permissions and resources in the form of innovation toolkits, firms can not only outsource the innovation activity to customers and the various stakeholders, but also bundle them into their own overall product development process (von Hippel \& Katz, 2002; Bogers \& West, 2012).

\subsubsection{Tool-kits to Outsource Innovation}

Innovation tool-kits are a set of user-friendly resources including design instruments, knowledge libraries and simulation environments that enable the user to develop a new and customized product to use (Parmentier \& Gandia, 2013; von Hippel \& Katz, 2002). Simple Internet technologies such as forums and social networks may be part of tool-kits as a channel of information sharing and collaborative design input (Parmentier \& Gandia, 2013). Von Hippel (2001) outlines five key characteristics tool-kits require being effective. Firstly, they must allow a user to learn, via trial-and-error process, how to develop their solution. Secondly, the toolkit must have a "solution space" where the user can design and possibly test their solution. Thirdly, the tool-kit should be simple enough to be operated by the existing level of skill users have without the requirement to learn new skills or languages. Fourthly, it should provide knowledge libraries with basic and 
commonly used features as to focus the users' efforts on their specific solution design rather than the design of its elements. Lastly, tool-kits should limit the user to designing solutions that are both possible and ideally require no further conversions to be produced by the firm.

Firm providing the tool-kit must put constraints to only permit solutions that can be produced at reasonable prices and requires, if any, few changes to the existing manufacturing process (von Hippel \& Katz, 2002). These constraints bound the degrees of freedom that the user is allowed work within to change and modify the existing solution into a new and affordable product. By implementing the constraints, firms unstick their knowledge and shift it to users to implement in their solutions (von Hippel $\&$ Katz, 2002). This is because the firm tends to know more than the user about the most effective manufacturing process while the users know more about their needs and how they use the product (Franke \& von Hippel, 2003).

To gain first-mover advantage and set the standard, firms must design a tool-kit based on their own capabilities and introduce it into a user community. A firm may design their first generation tool-kit off their in-house design toolsets and modifying them into a userfriendly system (von Hippel \& Katz, 2002; Franke \& von Hippel, 2003). This way, the firms quickly produces a tool-kit that not only incorporates its manufacturing process but also creates easy transitions of user solutions to firm language. The source code of the tool-kit and the internal capabilities of the firm remain concealed granting partial access 
to alter and view content (Gassmann, 2006; Laursen \& Salter, 2006).

In order to be an effective, the tool-kit must provide minimalistic tools and resources to enable creativity and solution building (Parmentier \& Gandia, 2013). Once introduced, firms can now encourage its usage by organizing contests of varying degrees from simple idea generation to full design solutions (Hutter et al., 2011; Füller \& Matzler, 2007).

\subsubsection{User Communities: Source of Innovation via Tool Kits}

The most effective way to maximize output from users through the tool-kits is to embed it into a user community. User communities are a group of users of a particular good (product or service) and engage in trading, sharing and disseminating information about that good (Parmentier \& Gandia, 2013). Within these communities can be found active members who innovate by creating new functionalities or content that meet other users' needs (Prügl \& Schreier, 2006). These innovative members are known as lead users and often develop products that maybe not only solve the needs of others but may also be new to the industry (Baldwin et al., 2006). But, user communities and their members do not focus on sustainability of their innovations and knowledge (Bogers et al., 2010).

Once a tool-kit is introduced, the whole user community can participate in innovation making them an innovative user community (Sawhney \& Prandelli, 2000). For this 
reason, firms utilize user community as a strategic asset (Hienerth et al., 2012). Because these communities are established before and without firm guidance, firms need to inaugurate themselves by supporting user communities via financing and or offer website hosting (Parmentier \& Gandia, 2013). The disconnection between the firm and user community puts more emphasis on the need to work closely with community managers to monitor innovations and implement them into new versions.

Interestingly, tool-kits are multipurpose and can be used by the firm to define the dynamics between the user community and themselves (Parmentier \& Gandia, 2013). Through the tool-kit, the firm becomes connected to the web of users and stakeholders and thus establishes a consolidated network. The firm now may assign responsibilities and roles between the members to form development teams, processes and guarantee involvement. After the users are assigned roles, provided with the resources to innovate and are connected together through the tool-kit, the user community becomes autonomous over time (Prügl \& Schreier, 2006). New ideas, products or uses of existing ones can now be harvested from the user community having already been discussed, evaluated and iteratively improved by other members (Parmentier \& Gandia, 2013; von Hippel \& Katz, 2002).

Through the use of innovation toolkits, firms can assign the sticky need-related tasks to users, the sticky production-related tasks to the other stakeholders and members can draw on each other's knowledge to develop a product with compressed times and cost (von 
Hippel \& Katz, 2002). However, the innovation process through tool-kits is not entirely a costless endeavor. The modification, creation and approach the users and stakeholders take to innovate are limited to their perceived benefits versus their perceived cost. The efforts involved in obtaining and learning the use of the tool-kit plus the time and effort of designing/implementing a solution is a heavy cost placed on the members (Franke \& von Hippel, 2003).

\subsubsection{Motivating and Managing Contribution}

Different profiles exist among the mass of the innovative user community making a diverse set of sub-groups varying in skill-level, know-hows, interests and needs (Bonaccorsi \& Rossi, 2003). Large subsets of innovative users are not capable of designing a solution but simply using and suggesting improvements. According to Bonaccorsi and Rossi (2003), users who design - often the lead users (Bogers \& West, 2012) - are in many cases hobbyists who have the technical knowledge and drive to develop new technologies in their spare time. Initially, a firm and community need only to rely on a set of lead users (Jeppesen \& Frederiksen, 2006), but the involvement of other members add complementary value to further the development process and reduce the perceived cost of other members. Those who do not design, but only use the innovations, help test the product and provide feedback that aids in iteratively improving the design (Schau et al., 2009). 
Different types of members within a community may use a single tool-kit (Parmentier \& Gandia, 2013). Thus, to successfully achieve effective output, firms must steward to the community. First and foremost, there must exist mechanisms of motivation to encourage user involvement in addition to the intrinsic factors. Intrinsic motivation is the internal gratification a member receives from participating or achieving a goal within the community. These gratifications may be compared to the intellectual pleasure one feels at making a scientific discovery (Bonaccorsi \& Rossi, 2003). Mechanisms of motivation often take the form of extrinsic forces that encourage participation regardless of intrinsic presence. Examples of extrinsic motivation are recognition by the firm (Jeppesen \& Frederiksen, 2006), peer reputation (Hertel, Niedner \& Herrmann, 2003), monetary incentives (Jeppesen \& Lakhani, 2010) and reciprocity of solutions. Unfortunately extrinsic motivation is non-sustainable requiring both resources and renewal (Parmentier \& Gandia, 2013).

Leadership within the user community plays a significant role in the effectiveness of innovation output. Through proper leadership, intelligent steps can be taken to steer the evolution of projects and choose the best fitting solutions. To be successful, a hierarchical governance structure needs to be in place allocating roles and tasks to the members (Bonaccorsi \& Rossi, 2003). Hierarchical coordination, however, may dispirit the intellectual creativity as well as the intrinsic motivations associated with innovating.

For this reason, roles cannot be strictly assigned and no member can be forced to perform 
a task. Instead, a less formal set of headship based on reputation and shared conventional rules can help govern the members in a more communal fashion (Evers, 2000). A new bottom-up hierarchical structure often is formed based on the members with significant contributions and reputation (Bonaccorsi \& Rossi, 2003). Therefore, in addition to the tool-kit, firms can assign their own employees to participate within the community (West \& O’Mahony, 2008). By contributing their skills and efforts and monitoring the community's activities (Parmentier \& Gandia, 2013), employees become part of the bottom-up hierarchy keeping the firm in control.

\subsubsection{Benefits of User Innovation}

The strategic use of user innovation in this manner positions the firm with key assets that serve also as benefits to their consumers. During firm's involvement in the community, lead users invest significantly more effort into participation in order to obtain recognition and prestige from the firm (Bonaccorsi \& Rossi, 2003). This not only results in increased innovation output but also offers firms a channel from which they may find new talent. The innovators also reap benefits of meeting their own needs and earning profits from extrinsic payments or intrinsic gratification (von Hippel, 2007). Consumers (both innovators and non-innovators) also gain greater satisfaction from firms becoming repeat customers thanks to the more specialized solutions that the tool-kits and user community offers them (Franke \& von Hippel, 2003). 
According Morrison et al. (2004), through the user community and tool-kit, firms can disseminate their new products more effectively. Research has shown that user developed solutions are easier to adopt by consumers because of their easy use. Additionally, since lead user are opinion leader as well, they not only help innovate but encourage adoption among other members and the general public (Franke \& von Hippel, 2003).

\subsubsection{Business Appropriation of Innovation User Communities}

The most common problem firms are faced with when utilizing user innovation is creating a business model to appropriate profits from user innovation (Franke \& Shah, 2003). Innovations sprouting from users and their communities entail free disclosure (Lakhani \& von Hippel, 2003). Firms will source new products from the users and only commercialize finished products (West \& Gallagher, 2006). If the firm does not obtain consent from the user innovators, it may run into intellectual property (IP) conflicts (Pisano \& Teece, 2007).

Management of IP is central to controlling knowledge and determining ownership of the innovation (Bogers \& West, 2012) especially since strong IP regimes by the firm can retard the innovation spirit of the user community (von Hippel, 2005). Private business models can attempt to solicit license agreements from the innovators (West \& Gallagher, 2006). Instead, firms can align the user community around ideas that belong to the firm and thus legitimize their claim to the innovations ownership (Jeppesen \& Frederiksen, 2006). Alignment of firm and community strategies around firm IP and tool-kits (von 
Hippel \& Katz, 2002; Jeppesen \& Frederiksen, 2006) simplifies the appropriation models (Dahlander \& Magnusson, 2008).

The use of user innovation may stimulate new types of business models to appropriate profits. Firms may opt to provide a free product as a solution to attract significant adoption among consumers, and then make money by selling upgraded versions or addons that are developed within the user communities (Parmentier \& Gandia, 2013). This new lock-in mechanism helps establish user communities where complementary products are developed and the viscous cycle repeats itself off furthering the products use. Corresponding services including consultancy, maintenance, updating and training (Bonaccorsi \& Rossi, 2003) can now be a source of income for the firm as well as confirm to the consumer the fundamental reliability and usability of the product. These services institute an added revenue stream and support for the tasks user communities often do not find appealing to solve (Bonaccorsi \& Rossi, 2003).

\subsubsection{Conclusion of User Innovation}

It is evident that harnessed user innovation becomes one of the firm's most valuable resources (Parmentier \& Gandia, 2013). Firms can source innovation from users, seed the community with spin off products that cannot be commercialized by the firm (Bogers \& West, 2012), or use users as a form of peer-to-peer support (Franke \& von Hippel, 2003). Thanks to tool-kits and user communities, firms now can produce more specialized and reliable products, create lasting relationships with consumers, and eliminate the physical 
and temporal constraints of the real world (Parmentier \& Gandia, 2013). However, alignment of strategies between firms, stakeholders and user communities poses difficult terms over long periods (Parmentier \& Gandia, 2013).

The alignment of the members is a particular challenge because of the varying interests among members and the limited resources possible to be put towards extrinsic motivational mechanisms (West \& Gallagher, 2006). Additionally, Prügl and Schreier (2006) found that keeping the sub-groups participating, different tools needed to be supplied. Lastly, the virtualization that Internet technologies deliver and enable user decentralized user innovations, also distributes the materialization in the physical world (Parmentier \& Gandia, 2013). Parmentier and Gandia (2013) argue that the physical world fosters better understanding of products and stronger ties among innovative members.

\subsection{Co-creation}

Unlike simple user innovation where firms tap into user communities for improvements and ideas on their products via the use of tool-kits, contests and jams (Chen et al., 2012), co-creation does not necessarily link to the core offerings of the firm (Saarijarvi, 2012). Instead, co-creation extends the user innovation process into the entirety of the value chain from product development to end shopping experience in a way that reduces a firms labor costs and increase customer convenience. Co-creation does not simply aim to 
further enhance or specialize a firm's product, but is a means of appropriating new ideas or approaches from users and other stakeholders to create new values and/or experiences (von Hippel et al., 2011). It engages stakeholders, users and consumers in a collaborative organization to develop a "we" competency rather than a differentiated "you" and "I" interaction (Lee et al., 2012; DeFillippi \& Roser, 2014).

\subsubsection{Co-Creation Community}

Chesbrough (2003) refers to co-creation innovation as "open-innovation" because it works off an ecosystem of complementing stakeholders that combine their efforts into a coherent collaboration to create solutions in different areas of the value chain. It originates from collaborative social networks where individuals shared resources such as digital music in peer-to-peer networks (Kannan et al., 2000; Asvanund et al., 2004). Other Internet enabled social networks such as open-source software, internet-based voluntary support groups answering question also helped stem the idea of sharing resources to benefit each other (Chen et al., 2012).

In most cases, firms will outsource the co-creation process to external agencies or communities to develop complementing solutions (DeFillippi \& Roser, 2014). This complementing collaboration lets firms and other stakeholders to work together rather than competing, consolidating not only resources but also a larger market segment over a distributed network (Gassmann et al., 2010). 
Zwass (2010) distinguishes two types of co-creation: sponsored (direct benefit to firm) and autonomous (indirect benefits to firm). Sponsored co-creation leads individuals, communities and/or other stakeholders to perform complementary innovation activities at the request of a firm or organization. In contrast, autonomous co-creation is the result of individuals, communities and/or stakeholders undertaking complementary innovation activities independent of a firm or organization although they use platforms or products specific to firms.

The main contributors in sponsored co-creation are often the customers and users of the sponsoring firm products. The firm will use Internet technology to connect to and work with their consumers across geographical and time zone locations (Chen et al., 2012). Wiertz and Ruyter (2007) found that firms reach out to their consumers first because they already have an established bond. Although sponsored, the co-creation process can grow past the firm-customer boundaries and involve many other stakeholders to create a manyto-many relationship (Mele et al., 2010). The stakeholders can be other firms, research institutions and not-for-profit organizations that can supplement the co-creation with indepth knowledge and capacities (Russo-Spena \& Mele, 2012). Pooling together knowledge, experiences, and resources from a heterogeneous mass of participants in cocreations allows for a strong interchange of ideas and innovations that fosterers the production of creative solutions (Russo-Spena \& Mele, 2012). 


\subsubsection{Benefits of Co-Creation}

There are several benefits that arise from participating in co-creation activities other than leveraging innovative ideas for new products, services or even new ventures. Lee and colleagues (2012) state that co-creation helps improve the architecture of products (possibly resulting in better quality) and lowers the cost because of the shared knowledge of module integrations and manufacturing processes. Likewise, due to the parallel nature of development that is inherent to collaborative production (Russo-Spena \& Mele, 2012), the product life cycle is shortened allowing for faster launch and increased speed to market (DeFillippi \& Roser, 2014). Moreover, with a diversified collaborative network supporting simultaneous innovations, firms and other organizations in the co-creation network become more efficient and agile, capable of scaling up rapidly (Adler et al., 2011).

DeFillippi and Roser (2014) identified several advantages to using co-creation networks. These advantages include the lower risk of market failure: with better communication of needs between customers and stakeholders as well as product optimization through each other's knowledge and complementing offerings, there would be a lower likelihood of customers seeking out competitive solutions. In turn, this will lead to higher rates of word-of-mouth marketing as a result of the customers' all-encompassing satisfaction from the specialized assortment of solutions offered. Having customers and other stakeholders as partners, it becomes easier to learn (from shared knowledge) of failed or less successful offerings and better understand the needs that should be focused upon. In 
this fashion, firms and other organizations can concentrate on customer needs, experiences and relations rather than on the solutions themselves, opening the value chain to more abstract additions such as service interfaces or even retail atmosphere (Saarijarvi, 2012).

\subsubsection{Outsourcing Innovation in the Value Chain}

Zhang and Chen (2008) identified four areas in which to involve customers and stakeholder's participation in co-creation activities. Primarily, firms should involve the participants (specifically customers) in their selling mix capacity. Vargo and Lusch (2006) argue that a key co-creation role for customers, other than design, is the establishment of pricing, distribution and promotion of the products. Secondly, service care assistances (by either participant) that support the products can help build customer relations, distill customer difficulties and gain insights into product modification requirements. Thirdly, participants can co-generate new product development as discussed earlier. Lastly, they can be included in the labor force as partial employees as found in retail stores by the implementation of self-scanners (Saarijarvi, 2012).

To best implement a co-creation project and harness external collaboration a firm must first establish an internal culture of collaboration (Lee et al., 2012). After which, a firm should be able to answer important questions such as: what is the purpose of the cocreation project? Who will be involved? What part of the value chain will be influenced? 
How long will the collaboration be? And what motivational mechanisms will be required? (DeFillippi \& Roser, 2014) Understanding and managing co-creation initiatives are important to its success. The handling of the co-creation project will affect the participant's perception of the firm's objectives and efforts that in turn impacts member involvement (Porter \& Donthu, 2008). Done correctly, firms can promote positive beliefs and trust between it and the customers or stakeholders encouraging the members to cocreate with the firm. Wasko and Faraj (2000) suggest nurturing a common goal to unify the members and found generalized benefits implying that they may not be received directly for participating but will in the near future. Often, the firm should try to align the co-creation projects to their strategic priorities (DeFillippi \& Roser, 2014).

\subsubsection{Managing Motivation for Contribution}

Like user innovation, customers who participate in co-creation may not receive direct social or economic value (Chen et al., 2012). Instead, intrinsic factors play a significant role in motivating participation (Fang \& Neufield, 2009). The satisfaction of one's own needs for pleasure or enjoyment (Fuller et al, 2007), a sense of belonging (Zhang, 2010), or potential career advancement (Wakso \& Faraj, 2005), all contribute to individual members participating in co-creation. For this reason, a firm should apply applicable motivational tactics that fulfill the intrinsic needs.

Feedback is the most deceptively simple technic that firms can utilize for motivation. 
Research has found that individuals motivations where highly influenced by firm feedback (Jeppesen \& Frederiksen, 2006). Idea submission rates increase as a result of feedback and more importantly the speed of the feedback (Chen et al., 2012). Because firm feedback is viewed as "official recognition" and is visible by all members, it has strong influencing role in assigning reputation levels and cultivating quality-based participation. Contribution that does not go unnoticed and receives feedback, enforces the belief that the invested effort of the member is valuable, satisfying their intrinsic needs making them more likely to contribute again (Lester et al., 2005). Firms will need to find a balance between providing positive and indifferent feedback results. Bandura (1977) (ref. Chen et al., 2012) argued that positive feedback promotes both higher-quality and increased numbers of contributions from members, while negative or indifferent feedback resulted in members ceasing contribution. While the urge to always use positive feedback is tempting, firms must keep perspective of justifying (for reputation sake) the true usefulness of the individual's contribution. The timely and sensible feedbacks of firms that help motivate and give guidance to the group (Fried \& Slowik, 2004).

Another area to manage is the group unity and interactivity. When the members of a cocreation project feel an identity-based belonging (similar goals and interests), they tend to commit more time, effort and preference to that co-creation community (Ren et al., 2007). Creativity in the idea generation likewise increases as the participants connect and interact with numerous members, cross-fertilizing knowledge and ideas allowing for the discovery of ideas that might not be apparent (Grewal et al., 2006). This does not imply that the members need to be densely interconnected with each other but rather that there 
should be an exchange of communication (Chen et al., 2012). Moreover, anonymity of members tends to hinder the unity and the interactivity among members. This is because feedback from peers also adds to the belonging and community feeling. When members experience teamwork through collaboration of effort or even simple feedback, the members will feel more competent and trusted to continue investing into the co-creation project. If the co-creators see their ideas actually accepted, acted upon and or used by others, it makes the value of participating in co-creation projects more apparent. The quicker the response time of from others, the stronger the sense of realness and belonging one gets. For this reason, firms should manage the co-creation project in a way to nurture oneness and timely interactivity.

\subsubsection{Co-creation Methodology}

Firms always need to find appropriate assortment of members (with different talents) in their project base to avoid having an unbalanced organization teeter tottering between firm influences and allowing novel contribution by the members. For such, Russo-Spena and Mele (2012) identified five areas in which members of a co-creation project can contribute: co-ideation, co-evaluation, co-design, co-test and co-launch.

Co-ideation is the phase where members of any background (consumers, fans, and partners) may propose innovative ideas to the community. In this phase, ideas for new or 
old products are proposed, discussed, refined and later voted on to determine which are most appealing. Similarly, co-evaluation is a phase that focuses on the appraisal of the proposed ideas. For a deeper understanding, firms can request reasons or comments in addition to the voting to allow members to explain the reasons for up voting or downvoting an idea. Once voting is completed, top management teams first review the ideas for their business potential and are then passed onto other teams to determine and weigh the cost and benefits of its implementation. If deemed affordable, the firm will announce potential timelines and methods to realizing the completion and commercialization of the idea.

The co-design phase is the implementation of the approved idea. This phase can include many different activities varying from drawing to constructing and even documenting. This phase requires that the sponsoring firm or governing team provide resources such as tool-kits and knowledge to enable the members to conduct their work and implement the idea. After construction, less tech-savvy members of the community can help continue the co-creation by offering their services to improving the prototypes. Before launching to market, this phase helps refine the new product and contribute feedback to its actual value of need. This phase involves iteration where the pre-commercialized product is tested, refined and presented again until it reaches satisfactory levels or abounded all together. The final phase is co-launch, where the product is released to market, and thanks to the co-creation group, will have already have early adoption and with a promotions force via word-of-mouth. 


\subsection{Living Labs}

The living lab concept builds upon the increasingly popular innovation concepts such as user-innovation and co-creation (Leminen et al., 2014). Cross-fertilization is the cornerstone in all innovation systems recombining dissimilar and external knowledge together to create novel solutions. In like manner to co-creation (Kusiak, 2007), living labs involve users as co-producers of innovation through the use of Internet technology; their role is re-interpreted from being an "end-user" who simply uses and customizes the product, to a patron that integrates the product into their social context. Unlike cocreation, living labs provide physical and organizational infrastructures in addition to the tool-kit software to enable user development (Guzman et al., 2013).

According to Liedtke and colleagues (2012), living labs are an innovation approach that optimizes both the production and consumption of products and services. It is by default a physical or virtual (Guzman et al., 2013) laboratory that analyses the technical and socio-economic attributes of innovations using its set methodology for solving people's needs and integrating with the person(s) environment (technologically and socially). Pierson and Lievens (2005) agree that living labs is an infrastructure based on a methodological innovation platform that combines users and researchers together to design, develop and evaluate innovations in the early stages of a products life cycle. They refer to it as a 'test and experiment action platform' that distinguishes it from test beds; an approach that focuses on controlled testing in a lab environment (Ballon et al, 2005). 
To best describe this innovation approach/platform, we will examine the defining attributes to better understand the multisided constructs of a living lab.

\subsubsection{Living Labs Objectives}

Living labs intend to deliver more than the other forms of innovation concepts. According to Kareborn and colleagues (2010), living labs propose to add value at various levels including economical, business and user tiers. As a concept that emphasizes realism, one of the main deliverables of living labs (for adding value) is the creation of an innovation arena (Almirall \& Wareham, 2011). The arena, or better known as test environment, is an infrastructure were human responses could be observed after introducing innovative solutions (Eriksson et al., 2005; Barcenilla \& Tijus, 2012).

In addition to the innovation arena, the living lab offers a methodology that helps coordinate the experimentation process that occurs within the test environment (Almirall \& Wareham, 2011; Schaffers \& Turkama, 2012). The methodology facilitates the research and sets attainable goals for experimentation completion. It guides the collaborative innovation into sensing, prototyping, validating and refining the solutions using real-life contexts (Barcenilla \& Taijus, 2012). These methodologies are continuously generated and refined from the knowledge produced by the lab (Kareborn et al., 2012). Leminen and his colleagues (2012) believe such methods are the primary output of the process versus the innovative solution itself. 
Knowledge generation is therefore an objective of living labs. The lab itself is indented to carry out research and development and experiment with products and services (Schaffers \& Turkama, 2012) and aims to continuously learn and evolve over time. The labs researchers are focused on producing scientific results (Kareborn et al., 2010). They collect data from users (Folstade, 2008) to study behavior and develop patterns that help predict outcomes for society (Kanstrup et al., 2010). Once the studies are complete, living labs compile and diffuse the knowledge accordingly (Almirall \& Wareham, 2011).

Part of the knowledge living labs aim to generate is "how users interact with products that bring real benefits to consumers" (Eriksson et al., 2005; Barcenilla \& Tijus, 2012). The function of such knowledge is to validate the innovation and ensuring initial demand for the product prior to commercialization (Almirall \& Wareham, 2011). Budweg and colleagues (2011) explains that this goal helps innovators balance new functionalities with user perceptions for optimal benefits and product adoption.

According to Kareborn and colleagues (2010), the listed above objectives point in the direction of a greater objective for business development. The intangible activities that help business develop and monitor their well-being are part of the living lab mandate. They therefore include employee support, supplier value, managerial tasks and societal value. Living labs will, through concrete projects and community building activities (Schaffers \& Turkama, 2012), help build business and make a social impact on the environment and economic domains. 
Living labs are also believed to be responsible for the facilitation of the ecosystem of partners; they mediate between the various actors for the production of successful outcomes, coordinate the activities to drive creation of products and services and continuously network to maintain critical mass for open collaboration (Almirall \& Wareham, 2011; Leminen et al., 2012). Living labs is a collaborative platform and requires the proper settings for open collaboration (Schaffers \& Turkama, 2012).

\subsubsection{Stakeholders and Actors}

Good living labs are based on the open involvement of many stakeholders in the innovation process and user-centric approach versus technology centered development (Nystrom et al., 2014). It is important to bring together various stakeholders and actors of various backgrounds, perspectives, knowledge and experiences to stimulate creativity and create new ideas for innovative products (Eriksson et al., 2005; Kareborn et al., 2010). The larger the variety of complementary firms and organizations participating - whose presence represents a more realistic social context - the better the findings. This is typically what is found in pre-established open innovation living labs; firms and organizations join forces to test their products and services ideas and form newer more applicable products (Pierson \& Lievens, 2005).

Living labs will engage academia, government, technology centers, SMEs, citizens, researchers, developers, service providers, users and many more to collaboratively undertake projects and iteratively develop and validate innovations together (Almirall \& 
Wareham, 2011; Barcenilla \& Tijus, 2012). Together they analyze situations, design and develop solutions that meet user needs, and access the acceptability and usability of the output as to how it impacts user behavior and its marketability.

As a collective, they achieve new boundary spanning knowledge and learning as they share their wealth of knowledge and expertise, and conduct research in real world contexts (Kareborn et al., 2010). Kanstrup and colleagues (2010) attribute the learning to the social contact and communication during the extensive activities of the lab that builds up a shared understanding of both, real needs and real solutions that can address these needs.

\subsubsection{Users}

Users play a key role in living labs as they do in all other innovation concepts; they are the source of innovation (Schaffers et al., 2007). In living labs, users are not seen as passive respondents (Schuurman \& De Marez, 2012), and they are more than an object for testing and feedback (Schaffers et al., 2007). Living labs are meant to go beyond user-centered research that simply incorporates users' knowledge and ideas through the use of methodologies and tools (Kusiak, 2007). Instead, users are viewed as partners, experts of their own experiences, and it is the intent of the lab to empower them by providing the required resources to convert their ideas into innovative products (Sanders \& Stappers, 2008; Dell'Era \& Landoni, 2014). 
Leminen and colleagues (2014) have identified five types of user involvement within living labs: informant, tester, contributor, co-creator, and creative consumer. Informants are users who are passively involved and viewed as a source; they often only provide information upon request. Testers are more involved within the lab as they invest their effort in the development cycle by engaging in the real-life test environment. Compared to testers, contributors not only get involved in the testing phases, but also help in the development of the innovation. The last two, co-creator and creative consumer are nearly indistinguishable. The users who fall in these categories are highly engaged in the full value chain, creating, developing and testing innovations. As such, they are respected and viewed as equals within the living lab.

Living Labs involve users because they help ensure that the innovations are desired, useful and usable (Kareborn et al., 2010). The innovation output of users is beyond that of other stakeholders because they are able to think passed constraints that designers would consider (Kanstrup et al., 2010). Moreover, users who engage in co-creation activities help reduce development cost for companies who are involved (Katzy, 2012). Living labs therefore put great importance on partnering with users to involve them in collaboration for producing and ultimately driving innovation (Almirall et al., 2012). Users get involved because they are seeking better solutions that will satisfy their needs (Leven \& Holmstrom, 2008).

\subsubsection{Small Firms and Designers}


The operations of living labs is often driven by industry partners who are large, small and medium sized enterprises and as well as entrepreneurs (Shaffers \& Turkama, 2012). The industry partners (with R\&D departments) often take on the role of designers and join living labs to access external ideas from others. Industry partners use the diverse collection of knowledge to not only form new innovations, but to renegotiate the use of their existing offerings buy opening up to new interpretations from the other partners in the lab (Almirall \& Wareham, 2011). They use the labs resources, networks and techniques to gain knowledge to develop solutions to new opportunities and to meet the needs of users (Leven \& Holmstrom, 2008).

Living labs provide an alluring innovation platform for small companies, because of its ability to provide relevant information to support innovation in real-life contexts (Niitamo et al., 2012). Kareborn and colleagues (2010) believes smaller firms join living labs because they lack the resources and knowledge to stay competitive and this network offers a competitive edge. Although their aim is for improved business opportunities, industry partners still give back by providing new products and services in addition to their own knowledge and support in the lab as a whole (Schuurman \& De Marez, 2012).

\subsubsection{Researchers}

Researchers are the other form of stakeholders. They may be part of academia, research centers or other institutions. Compared to the other stakeholders, researchers focus on the generation of knowledge, not products (Dell'Era \& Landoni, 2014). Researchers use their 
expertise and information to offer methodologies and technology support to improve the process of collaborative innovation (Leven \& Holstrom, 2008).

\subsubsection{Collaborative Culture}

One issue arising from the openness of living labs is the competing intellectual property (IP) of technology or business models from firms/organizations that are contributing to the infrastructure and test when state-of-the-art resources are pooled from many kinds of firms and organizations. As participants expose their competencies by sharing their resources and become a work force providing ideas, designs and research feedback, proper compensation needs to be present. Compensation methods can come in the form of appropriate IP sharing, reward mechanisms or incentive programs. The involvement of public administrators (i.e. government, law makers, etc.) is important to determine and set suitable legislation that allows the proper use of living labs. To date, public administrators have only been used for funding, but it is critical that their role go above and beyond to start reforming the basic interactions laws of society. A common collaborative culture and shared goal of innovation should be strived for to achieve best outcomes from living labs.

Because living labs brings together many stakeholders together, it is important for trust to be established in order facilitate the equal and fair exchange of knowledge, resources and efforts (Barcenilla \& Tijus, 2012). Genuine trust within the living lab is the corner stone that is needed (Niitamo et al., 2012). To be effective in innovations, living labs cannot 
favor firm specific product or business models (Eriksson et al., 2005). Collaborative projects require to first and foremost having an open mindset, at the individual and organizational level (Kareborn et al., 2010). Proper management of intellectual property rights may be needed (Niitamo et al., 2012) to prevent one member's interests from dominating in the innovation co-creation process, or the invested level of activity (Leminen et al., 2012). All stakeholders need to be appropriately managed to ensure fair collaboration without domination.

Ideally, the structure of the living lab would avoid dependencies or asset lock-ins that would hinder the capture of value created by and for all stakeholders. Guzman (2013) explains that the idea behind a living lab is to have a win-win strategy. A well-structured partnership and understanding of collaborative efforts is essential in preventing competition that would lead to greed and communication problems.

Unfortunately collaboration patterns and communication in living labs communities are seen as unstructured or ill-defined (Budweg et al., 2011). Budweg and colleagues have tried to apply top-down governance as both top-down and bottom-up forms have been observed. Leminen (2013) research has developed a governance framework to determine their use of top-down versus bottom-up approaches, however, it requires the categorization the living labs type into one of the four types: provider, utilizer, enabler or user driven. 
According to Leminen (2013) top-down is an authoritarian managerial method for hierarchical innovation where the process is "directed, controlled and precedes from top to bottom when creating, prototyping, validating, and testing and new technologies, services, products, and systems in real-life contexts". In contrast, Leminen defines bottom-up approach as an "emergent grassroots ideas and needs are collectively developed, created, prototype and validated for mutual and shared objectives". Leminen and colleagues (2012) pose that top-down is for managing the innovation process while bottom-up is for facilitating the innovation. In either case, clear responsibilities and ownership need to be defined, as they are critical to success. Without clear management, proper ownership of activities, the lab would risk failing or poorly executing tasks which would undermine the reputation of living labs as a system.

Katzy (2012) has determined that living labs often take on the form of autonomous legal entities, public-private-partnerships, or as independent units within larger organizations. A living lab being its own entity allows for the development of its own brand, team, organizational structure and resources. In contrast, its independence emphasizes the need for financial investment to execute its intended activities.

In like manner, living labs are also required to uphold ethical standards due to their approach of experimentation on/with users. Barcenilla and Tijus (2012) recognized that living labs share their objectives, research and outputs with all their members, especially at the conclusion of a study. Nonetheless, a professional code of conduct is required to define the whole set of rules that would constrain the activities of the lab. 


\subsubsection{Resource and Financial Characteristics}

Living labs require substantial financial support to operate the resources and extraneous activities of real-life experimentation (ENoLL, 2007). External funding from policy makers is used by the labs to aid in national policy objectives (Katzy, 2012). They can also receive funding from individual investors, venture capitalists and partner corporations. Living labs also have their own business models where they create financial income from their activities in order to fund their initiatives. Their business models include supportive services.

\subsubsection{Living Lab Methods}

As an ecosystem where real-life experiments are carried out with/on users, there is no doubt that living labs require operational methodologies (Schuurman \& De Marez, 2012). Development and test methodologies shape the competence profile of the living labs, and help in such things as acquiring user populations, accessing relevant knowledge and follow through on development (Katzy, 2012).

Management of this innovation process is a key to ensuring successful collaboration. Modeling the dynamic elements in the different phases of the living lab innovation cycle is the first step to understanding the influencing factors involved (Liedtke et al., 2012). A simple tool like running and managing a database to store and analyze information both 
generated or collected from stakeholders will not only support the modeling and good stewardship but also support knowledge development and information access and exchange. The data stored should include the contextual learning's, user-centered designs criteria, and early prototyping/testing procedures.

Almirall and colleagues (2012) identify four schools of thought that explain the degree to which a user is involved in the living lab innovation process. User centered methods passively involve the test subjects and measure such things usability, human factors, and ethnography patterns. Comparatively, participatory methods involve users as equals with the rest of the partners changing the process to a co-creation process. Design driven methods are established by designers and set procedures that direct the living lab studies to output solutions. Lastly, user driven methods set the user as lead and they direct the innovation process as they see fit. According to Eriksson and colleagues (2005), the living lab principle is not meant to use customers as mere "Ginny pigs" in product experimentation, but to access their knowledge relative to their social context. It is a

means to understand the users' situation and match the solution to the situational needs of their changing condition.

\subsubsection{Living Lab Procedures}

Pierson and Lievens (2005) proposed a four-step methodological research approach of living labs. The preliminary stage of innovation cycle is identifying and selecting the correct participants through the scientific usage of maximum variation sampling. This of 
course is done after the proper selection criteria are applied based on profiles to choose a sampling group of lead users. The first phase of the cycle is termed the contextualization or the explorative phase. In this phase, the end-goal innovation research is defined as well as gathering all necessary background knowledge required on the technological and social variables. A study is first conducted to map out current and future technologies and determine the socio-economic factors that would impact the research focus. The next main step is the "Concretisation" phase. This phase involves the thorough understanding of the day-to-day activities, their related behavior and prescription towards the introduced technology. Studying and observing the users usage of the technology is done in this phase by direct monitoring or by analyzing backlogs. Data processing of logs provides insight into usage but analysis of diaries and surveys from focus group are then implemented to investigate the motivation and explanation of certain behaviors. After implementing the actual test of the technology in a living lab setting, all this is left is the feedback phase. The goal of this phase is to examine the thoughts of users and examine the evolution of behavior/attitude towards the technology. Using this knowledge, the team can recommend changes to the technology to accommodate users.

Other researchers have mentioned various steps to take that complement the methods outlined by Pierson and Lievens (2005). The following is a compilation of suggestions from other researchers in regards to the ideation, design and commercialization tasks.

During the first step of idea generation, the lab can engage small focus groups and 
promote storytelling for detailed and information rich situations to determine problems and possible solutions (Guzman et al., 2013). According to Niitamo et al. (2012), the living lab should start by defining the intended market to which a solution will be formulated. Once selected, users should be consulted to gather perspectives and thoughts towards the target that provides a better understanding of what is felt when a particular product or service is used (Brown, 2008). In conjunction with fellow participants, such as designers and market experts, the shared comments can then reveal central themes and patterns that will direct the search for solutions (Kanstrup et al., 2010).

Ideas generated are evaluated for potential development. The use of scorecards and other indicators can be used to help narrow the selection (Edwards-Schachter et al., 2013). After determining the feasibility of the idea, specifications, requirements and restrictions are examined in the conceptual design stage. Stakeholders can choose how to stimulate and share designs forms: Mock-up paper prototypes, storyboard drawings, or other means (Guzman et al., 2013). Designers then implement the idea and its requirements into a testable and incremental changing prototype. The members can evaluate and suggest different improvements to be made.

Some have suggested that living labs should equip users with the proper tools so that they could design the solution (Leminen et al., 2012). However, according to Almirall and Wareham (2011), designs of solutions need to be renegotiated to focus on user emotion than the functionality itself. Using the knowledge generated from the lab, designs should 
be tailored towards addressing the usability and ergonomics of the product. In this fashion, products are improved at the functional and user experience level. Once the product is reaching maturity in the development cycle, Katzy (2012) suggests it be transferred to the participating industry partners and investors.

\subsubsection{Obtaining, Motivating and Training Participants}

The innovation research cycle within living labs starts by identifying lead users and stakeholders who are equipped with the capability to contribute to the innovation platform (Guzman et al, 2013). The focus is to connect and facilitate the partnerships between user communities, research organizations and firms. Effort must be invested to enhance the stakeholder roles to participate and intervene throughout the innovation activities in the living lab.

Users that participate in the living lab could be current or potential customers. They are randomly selected from consumer groups, lead user communities, members of research organizations or even firm employees (Niitamo et al., 2012). Once the participation population is selected, the lab must motivate contribution and could do so through competitions, pitting two groups against each other, or through monetary rewards.

Budweg and colleagues (2011) and Kanstrup and Christiansen (2009) suggest that the lab offers training to the necessary participants to both support and solicit feedback from the 
end users who are adopting the innovation. This tactic would be a cooperative effort to bridge the knowledge gap and help the iterative process in developing future products with the proper requirements. Such supportive activities should be included at all levels of operation because it creates interactive learning.

\subsubsection{Data Collection}

Part of the process is acquiring the generated data from the experimentations (Almirall et al., 2012) such that they are documented and used during the innovation process or at later time. Information about users and their usage patterns are often collected through the digital network (Leminen et al., 2012), which is later analyzed, by companies, government or other social agents to identify patterns and opportunities (EdwardsSchachter et al., 2013).

Eriksson (2005) views Internet Communication Technology (ICT) as the key competence of living labs. Through ICT, a firm can gain access to the diverse knowledge involved in the day-to-day context of consumer behavior. ICTs become central to living lab methodology and platform to enable research and development with users (Dell'Era \& Landoni, 2014). Having access to state-of-the-art technology such as service platforms and network access is significantly important in the interconnectivity of firms with users and their laboratory. The advanced interaction and interconnectivity between user, firm and social context is what differentiates it from the other innovation concepts. 
Monitoring and evaluating the living labs is a necessary part of management to continually analyze the practices, mechanisms, responsibilities and principles (cf. Leminen \& Westerlund, 2012) applied and to readjust or modify where needed to improve results or better commune partnerships. Monitoring communications processes and feedback loops between the members reveals the dynamics, feelings and state of participants (Liedtke et al., 2012).

\subsubsection{Collaboration Tools}

To enable collaboration, a portfolio of interconnected tools needs to be in place to allow for information exchange and communication (Budweg et al., 2011). The network consists of both synchronous and asynchronous tools that transcend geographical and time constraints. These tools are possible thank to the ICT and web technologies such as wikis, and blogs that offer shared workspaces (Schaffers et al., 2007; Budweg, 2011). Any form of web technology that can be hosted on servers allow for knowledge sharing and can facilitate cooperation and co-creating activities among the numerous stakeholders (Kanstrup et al., 2010; Kareborn et al., 2010). Additionally, data is also collected and managed through the use of traditional forms such as face-to-face interviews, and surveys (Edwards-Schachter et al.,, 2013).

\subsubsection{Benefits}


Living labs offers benefits to firms and other stakeholders similar to co-creation initiatives. Mulder (2012) identifies a few win-win strategies found in living labs among the stakeholders that are summarized in Figure 1 in List of Tables. Shared infrastructure composed of several resources including facilities, knowledge libraries, management procedures and design tools are pooled together by all the stakeholders and structured in a way to stimulate interaction for success (Guzman et al., 2013). Each stakeholder adds important resources to the multisided and complex context of living labs. The institutional support of the collaborating organizations helps reduce innovation failures (Pierson \& Lievens, 2005).

Table 2 Win-Win strategies for living Labs (Guzman, 2013)

\begin{tabular}{|l|l|l|}
\hline Type of Stakeholder & $\begin{array}{l}\text { Stakeholder Contribution } \\
\text { to Living Labs }\end{array}$ & $\begin{array}{l}\text { Living Lab Assets for } \\
\text { Stakeholder }\end{array}$ \\
\hline End Users & $\begin{array}{l}\text { Social knowledge of } \\
\text { services and products useful } \\
\text { for a user's community } \\
\text { Ideas for new products and }\end{array}$ & $\begin{array}{l}\text { Resources and partnership } \\
\text { networks to develop its own } \\
\text { ideas }\end{array}$ \\
& $\begin{array}{l}\text { Possibility of creating new } \\
\text { services } \\
\text { Participation in } \\
\text { development of products }\end{array}$ & $\begin{array}{l}\text { joint ventures with } \\
\text { companies }\end{array}$ \\
& $\begin{array}{l}\text { and services } \\
\text { Validation and enrichment } \\
\text { of technology }\end{array}$ & and research capability \\
& useful for developing new \\
\hline
\end{tabular}




\begin{tabular}{|c|c|c|}
\hline & & products \\
\hline Technology Providers & $\begin{array}{l}\text { Resources to create and } \\
\text { maintain innovation } \\
\text { infrastructure } \\
\text { Expertise and assets in } \\
\text { specific technology areas, } \\
\text { complementary with other } \\
\text { stakeholders, to provide } \\
\text { services to user } \\
\text { communities } \\
\text { Funding of initiatives } \\
\text { launched or developed by } \\
\text { lead users }\end{array}$ & $\begin{array}{l}\text { Community of active end } \\
\text { users to validate and enrich } \\
\text { product and services } \\
\text { Governance model to } \\
\text { manage to open innovation } \\
\text { cluster } \\
\text { Ethical and legal framework } \\
\text { Availability to open test- } \\
\text { beds } \\
\text { Availability to stable } \\
\text { network of complementary } \\
\text { stakeholders }\end{array}$ \\
\hline Public Agencies & $\begin{array}{l}\text { Policy framework to } \\
\text { support the development of } \\
\text { open and user-driven } \\
\text { innovation organization } \\
\text { Funding instruments to } \\
\text { support the initial } \\
\text { constitution of a living lab } \\
\text { Support to promote } \\
\text { innovation partnerships led } \\
\text { by user communities }\end{array}$ & $\begin{array}{l}\text { Support for the participative } \\
\text { definition of innovation and } \\
\text { economic and regional } \\
\text { development policies } \\
\text { New schemes to evaluate } \\
\text { innovation-related policies }\end{array}$ \\
\hline
\end{tabular}




\subsubsection{Innovative Ideas}

The innovation capacity of participating members increases significantly through living labs due to the cross-fertilization of knowledge and the open collaboration between various members (Kareborn et al., 2010; Dell'Era and Landoni, 2014). This concept allows for firms to outsource their innovation activities to a network of people and away from their employees (Howe, 2008) that may be over influenced by corporate culture. As mentioned before, the incorporation of user insights into the innovation process is a key success factor as it allows for significant learning of feelings and perspectives about customers usage of products. This learning is never ending and the living lab concept provides a means to continuously and systemically involve users (Moore et al., 2010).

\subsubsection{Networking Opportunities and Framework}

Living labs allow for partnering and networking opportunities; thus, members can access key market players, necessary research organizations and desperately needed business resources (Niitamo et al., 2012). As a public-private partnership, living labs often involve members who influence market conditions and policy regimes; this can be advantageous to small enterprises as they can position themselves to lower entry barriers to highly competitive and regulated industries (Almirall et al., 2012).

Research and development methodologies that guide the creation and validation of innovations it collaborative contexts are valuable to members (Eriksson et al., 2006). 
Additionally, frameworks that help in the design of products and services are also very attractive and are produced by living labs through their research (Niitamo et al., 2012).

\subsubsection{Market Development and Validation}

Living labs are not just developers of innovations; they are also the consumers of such development (Almirall \& Wareham, 2011). Part of the development process is the creation of the initial demand of the innovation through one of their networks. The labs help to solve commercialization concerns; they bring products to market through viable sales channels and a supporting product ecosystem (Bhide, 2008). Through the use of their real-life community, living labs create their future market (ENoLL, 2007).

Moreover, by integrating customers and providers into this form of development process, innovation outputs are less likely to fail; the ideas are sourced from and designed by users and complementing organization then tested and validated for market reliability (Eriksson et al, 2005). The real world environment they use as a test bed gives firms the ability to validate their ideas for effective and rapid commercialize of innovations (Leminen et al., 2012). Considering its real-life testing environment and user-centric philosophy, the living labs approach offers a highly innovative development system that produces relevant, reliable and sustainable products (Liedtke et al, 2012).

The research conducted by living labs alleviates the risk of launching new products by confirming its desirability and functionality. The living labs' advantage to address the 
user needs helps firms increase returns on their investment and time to market (Niitamo et al., 2012). Kanstrup and colleagues (2010) attribute these advantages to the test bed and community that creates a "critical mass" necessary for successful exploration, discussion and commercialization of the labs activities.

This benefit can be really attractive for small firms or start-ups that need to develop and prove their products desirability and relevance, which aid in securing venture capital.

\subsubsection{Financial Mitigation and Access to Resources}

Living labs help mitigate the financial burden on small firms through the use of national, international and private funding to support product development (Almirall \& Wareham, 2011; Niitamo et al., 2012). Moreover, with their strategic methodologies and network, living labs allow for cost-effective development because they avoid costly activities and mistakes (Schuurman \& De Marez, 2012; Katzy, 2012). At the user level, they get to access technology, facilities, training courses, dedicated personnel and other assets in the living lab to participate and add value to the research project (Pierson \& Lievens, 2005).

\subsubsection{Effective and Efficient Processes}

Collaboration results in improved processes and services and new business models (Edwards-Schachter et al.,, 2013). Research conducted with living labs often yields more 
accurate and higher results because it is not conducted in controlled environments (Schumacher \& Niitamo, 2008). Moreover, the research conducted is supported at all phases of the development cycle (Niitamo et al., 2012). The freed resources from R\&D by the firm can now be reallocated to drive other business related activities such as marketing and growth. Most importantly, all process required to effectively launch and operate a business is simplified and provided through the living lab's network and operations.

\subsubsection{Better Products and Knowledge}

Both Liedtke et al (2012) and Eriksson et al (2005) agree that innovation outputs from living labs benefit the user through the attention to ergonomic and interface design on the products that seamlessly integrate them into the user's daily life. Researchers also benefit from living labs. According Liedtke et al (2012), living labs gives researchers the chance to map how users experience and consume the resources around them. This gives insight into the discrepancy (if any) of users awareness and action about their resources. How things such as physical design, ease of use, pleasure in use, associates social norms and financial factors influence the usage of products.

\subsection{Constructs Synthesized from Literature}

User innovation, co-creation and living labs are useful innovation initiatives having both similarities and differences that make them unique. Table 3 summarizes the three 
innovation initiatives in a comparative format based on stakeholders, objectives, governance, tools, motivation and business appropriation.

In comparing the three innovation initiatives, a trend immerges in revealing progressive complexity of each consecutive innovation process. In some aspect, it might be possible that each innovation initiative consecutively builds on the other in scope of stakeholders, innovation objectives, and tools used to achieve a new way of appropriating value for the firm.

Table 3 Emergent constructs comparing innovation initiatives

\begin{tabular}{|c|c|c|c|}
\hline & User Innovation & Co-Creation & Living Labs \\
\hline Stakeholders & $\begin{array}{l}\text { - Users/communities } \\
\text { - Sponsoring Firm } \\
\text { - Manufacturers } \\
\text { - Suppliers } \\
\text { - Research Institutions }\end{array}$ & $\begin{array}{l}\text { - Sponsors } \\
\text { - Manufacturers } \\
\text { - Suppliers } \\
\text { - Users/communities } \\
\text { - Complementary Firms }\end{array}$ & $\begin{array}{l}\text { - IT providers } \\
\text { - Manufacturers } \\
\text { - Suppliers } \\
\text { - Public Administrators } \\
\text { - Research Institutions } \\
\text { - Users/communities } \\
\text { - Complementary Firms } \\
\text { - Competitive Firms }\end{array}$ \\
\hline
\end{tabular}

\begin{tabular}{|c|c|c|c|}
\hline Objectives & $\begin{array}{l}\text { - Innovation aims to } \\
\text { customize or improve } \\
\text { existing product }\end{array}$ & $\begin{array}{l}\text { - Aims to build } \\
\text { complementary products } \\
\text { and services } \\
\text { - Enhance architecture of } \\
\text { existing and future } \\
\text { products through } \\
\text { modulation and } \\
\text { outsourcing of different } \\
\text { components } \\
\text { - Building a network of } \\
\text { supportive products and } \\
\text { services for a complete } \\
\text { solution } \\
\text { - Accelerate } \\
\text { commercialization of } \\
\text { products }\end{array}$ & $\begin{array}{l}\text { - Harmonizing stat-of-the- } \\
\text { art technology with } \\
\text { market conditions and } \\
\text { societies needs } \\
\text { - Sensing needs, behavior } \\
\text { and perceptions of } \\
\text { innovation for } \\
\text { optimizing existing or } \\
\text { the development of a } \\
\text { new functional and } \\
\text { adoptable product } \\
\text { - Facilitate collaboration } \\
\text { between stakeholders } \\
\text { - Business development }\end{array}$ \\
\hline
\end{tabular}




\begin{tabular}{cl}
\hline Governance & Firms sponsor and \\
& govern the innovation \\
& process by implanting \\
& employees into the \\
& community to \\
& influence goals and \\
& direction \\
& Tasks are assigned \\
& based on users skill \\
& level (ideas only, \\
& development, \\
& feedback) \\
& Governance is bottom- \\
& up structure with firm \\
control over tool-kit \\
and employee members
\end{tabular}

- Firms sponsored or Autonomous

- Tasks are assigned in different areas of the development process (idea generation, evaluation, design, testing, commercialize)
- Up-Down (Combination of bottom-up and Topdown)

- Who assigns tasks are unclear

- Lab is own entity

\begin{tabular}{lll}
\hline Tools & Tool-kits are main & $\bullet$ Open Source tools and \\
technology used. & libraries \\
Enables designing and & $\bullet$ Use of internet \\
simulating innovations. & technologies and social \\
Also used as central & platforms \\
place for governance & $\begin{array}{c}\text { Could use provided tool- } \\
\end{array}$ & kits
\end{tabular}

- Use of internet technology for organization, communication and data collection

- Use of test beds for experimentation

- Various resources supplied by organizations

\begin{tabular}{|c|c|c|c|}
\hline Motivation & $\begin{array}{l}\text { - Users are intrinsically } \\
\text { motivated to obtain } \\
\text { recognition and } \\
\text { customized solution. } \\
\text { Extrinsic motivation } \\
\text { includes monetary } \\
\text { rewards. }\end{array}$ & $\begin{array}{l}\text { - Users are intrinsically } \\
\text { motivated to obtain } \\
\text { recognition from firms, } \\
\text { loyalty, enjoyment and } \\
\text { sense of belonging }\end{array}$ & $\begin{array}{l}\text { - Gamification } \\
\text { (Competitive tasks) } \\
\text { applied to stimulate } \\
\text { contribution and } \\
\text { creativity } \\
\text { - Extrinsic motivation } \\
\text { includes monetary } \\
\text { rewards and free } \\
\text { services } \\
\text { - Firms = Validation of } \\
\text { products }\end{array}$ \\
\hline $\begin{array}{c}\text { Business } \\
\text { Appropriatio }\end{array}$ & $\begin{array}{l}\text { - IP protection over core } \\
\text { components } \\
\text { - Open source licensing }\end{array}$ & $\begin{array}{l}\text { - IP protection over core } \\
\text { components } \\
\text { - Open source licensing }\end{array}$ & $\begin{array}{l}\text { - IP unclear } \\
\text { - Lab creates its own } \\
\text { funding through services }\end{array}$ \\
\hline
\end{tabular}




\begin{tabular}{|c|c|c|c|}
\hline & User Innovation & Co-Creation & Living Labs \\
\hline $\mathbf{n}$ & $\begin{array}{l}\text { for collaborative parts } \\
\text { - Value added } \\
\text { enhancements } \\
\text { - Consultancy } \\
\text { - Maintenance }\end{array}$ & $\begin{array}{l}\text { for collaborative parts } \\
\text { - Support Contracts } \\
\text { - Increased Market size } \\
\text { - Lower product cost }\end{array}$ & \\
\hline
\end{tabular}

Using the evidence from the comparison, the constructs have been defined as follows:

(1) Stakeholders: Parties who are involved in the innovation process regardless of the receipt of benefits. This definition is comparable to traditional terms in which individuals or parties have an "investment" within a project. As seen from Table 3 , stakeholders in the innovation process can vary from individual users, to suppliers, to public administrators and even to competitive firms.

(2) Objectives: The advantageous benefits of the output from the innovation process. The benefits can be viewed from the perspective of the product, the firm or the process.

(3) Governance: The manner in which the decisions and activities of the innovation process is decided and controlled. The governance includes both the organizational decision structure and the criteria for undertaking a specific activity.

(4) Tools: The necessary resources and specialized equipment required to carry out the innovation activities. Instruments used for managing the process, enabling design/manufacturing and testing the result are all considered tools. 
(5) Motivation: The reason for which the stakeholders engage in the innovation process and the techniques used to encourage participation/contribution from the stakeholders. As seen from Table 3 the techniques used to motivate and give reason to stakeholders to participate can be intrinsic in nature or extrinsically inducing.

(6) Business Appropriation: The means by which the innovation process captures monetary value from the innovation outputs. Capture of monetary value as evident from literature can be direct from the innovation output through its sale or licensing thanks to controlled IP. However, there are other means such as providing upgrades, maintenance, support services, penetrating larger markets and even reducing costs. 


\subsubsection{Lessons from User-Innovation}

The objective of utilizing user innovation is primarily (but not limited to) the evolution of a firm's existing product. User-innovation aims to customize a product/service to a user's needs or improve the functionality or design of the product. In this manner, as most novelty is dependent on the firms core product, firms have easier control over user derived IP. Likewise, by providing tool-kits, which will enable the stakeholders to design and simulate the product, the firm takes control of governing the innovation process using extrinsic rewards to complement the intrinsic motivation that users seek (recognition by firm and customized solution).

\subsubsection{Lessons from Co-Creation}

Co-creation innovation initiative aims to not only advance a firm's product, but to build an ecosystem of complementary products and services to provide a complete solution. Firms will bring in manufacturers, suppliers, users and other firms to pool their efforts and core components into creating novel solutions. Additionally, all the stakeholders can use their knowledge to help optimize the design of products by building a better architecture that is more efficient and open. In this scenario, sponsoring firms of cocreation innovation cannot appropriate revenue from all the innovations but instead win from the licensing of core components and the increase in sales of their product that is now used or supported by other products/services. 


\subsubsection{Lessons from Living Labs}

Living labs represent the most collaborative concentrated innovation process. It involves IT providers, manufacturers, public administrators, research institutions, users and competing firms. Its objective is to output novel products that have been developed with state-of-the-art technology under the conditions of market trends and societal needs. It achieves such a goal through "test beds" where users' needs and market trends can be sensed and the stakeholders develop and validate their products with users in the tested. It is unclear how much resources are pooled in addition to test bed infrastructure or the exact motivational factors that drive the innovation process. Likewise, with an extensive group of stakeholders and IP conflicts, appropriation of value by firms (other than knowledge) and governance methodology are not defined. 


\section{Chapter: Methods}

Chapter 3 describes the method used to construct and produce the deliverables of this research. The chapter is organized as follows; Section 3.1 (Approach) describes and justifies the use of a case study research approach in combination with content analysis, Section 3.2 describes the unit of analysis, Section 3.3 outlines the study period and Section 3.4 lays out the undertaken steps to complete the research.

\subsection{Approach}

Case study research is often undertaken when little is known on the topic (Eisenhardt, 1989), which is the situation of living labs. This is in agreement with Yin (2003) who stated that a case study research approach should be undertaken when the boundaries between the phenomenon and context are unclear. According to Baxtar and Jack (2008), case study research approach will help facilitate the exploration of the Living Labs phenomenon allowing for multiple facets to be revealed and understood through qualitative data. Additionally, this approach allows for the qualitative data that is readily available by ENoLL, to be combined and analyzed under the ideal of no theory or hypothesis to test (Eisenhardt, 1989). The explorative nature of this research uses such a process in discovering the underlying variables in the cases that will help develop the theory (Yin, 2003). In this situation, a case study approach would yield theory that is both unified and grounded in practice (Eisenhardt, 1989). 
According to Eisenhardt (1989), it is not uncommon for researchers to develop theory by combining observations from previous literature with common sense and experience. However, because of the confusion that surrounds the use of such inductive logic, it is preferred to combine various forms of qualitative data from several case studies to legitimize findings (Baxtar \& Jack, 2008). Combining several case studies is referred to as "multiple case study"; this approach allows for a comparative examination to understand the similarities and differences between cases (Yin, 2003). Moreover, the use of multiple research methods would significantly improve the validity of the conclusions as it reduces the susceptibility to biasing (Boyer \& Swink, 2008).

In addition to case study research, content analysis will be used on the text generated from the cases. Content analysis is a systematic technique that is used to evaluate qualitative content by converting textual data into a quantitative form that can be subjected to statistical analysis (Wolfe et al., 1993). The resulting output of the combination (case study research and content analysis) is a quasi-qualitative experiment.

Content analysis is a viable empirical method (Tangpong, 2011) that is advantageous in this type of research. The malleability of this method allows its use for the analysis of any form of data (Harwood \& Garry, 2003). It can be used on magazines, published articles, case databases, surveys, and questionnaires; this allows the researcher to utilize various and alternative data sources. As mentioned earlier, content analysis also provides researchers with a systematic procedure to classifying and analyzing qualitative data through its conversion to quantitative form (Wolfe et al., 1993). It is also a reasonable 
efficient technique for saving time and effort in collecting and analyzing data (Babbie, 1995) when used on preexisting data sets. Most importantly, content analysis methodology allows for repeatability of the research with iterative enhancements for the better reliability (Tangpong, 2011).

There are a few disadvantages with content analysis when combined with case study research. The primary obstacle is the analysis of large volumes of case data. Although larger data sets increase the rigor of the research, the manual pre-editing required prior to the content quantification, will require more manpower and/or processing time making the analysis a time consuming and labor intensive activity (Sonpar \& Golden-Biddle, 2008). Another constraint of the method is the validity of the results if the input data was not accurately recorded or processed putting into question the legitimacy of the findings (Tangpong, 2011). Lastly, inherent to this type of research (case study research), the interpretation of the data is subject to the researcher biases. This bias can be mitigated

provided a systematic coding process is used (Kolbe \& Burnett, 1991; Krippendorff, 2004).

\subsection{Unit of Analysis}

The unit of analysis at the case study research level is the phenomena being examined (Miles \& Huberman, 1994) which here are the attributes of the living labs. These attributes are specified constructs that were identified from literature for the purpose of shaping the theory and include: Stakeholders, Objectives, Governance, Tools, 
Motivations and Business Appropriation. These attributes are not guaranteed to be found during the analysis of the individual living lab data. However, the constructs form a set of propositions that help limit the scope of the research and ensure feasibility and completion (Baxtar \& Jack, 2008). Stake (1995) agrees that such propositions are necessary elements that help guides the research.

\subsection{Study Period}

This research formally began in March 2014 following the approval of professors in the TIM program. However, the research captures previous, publicly available data of living labs collected by ENoLL between the years of 2011 and 2012.

\subsection{Research Steps and Methodology}

Table 4 outlines the steps carried out for the research in accordance with the case study approach as described by Eisenhardt (1989). The steps are further elaborated in the subsections below.

Table 4 Step-by-step methods with undertaken activity

Step* Activity

1. Definition of Research Question

- Examination of salient literature regarding current innovation methods

- Choosing of common constructs apparent in theory 
population

- Cases restricted to $2011 \& 2012$

3. Multiple data collection methods

- Only cases with a profile and application form

\begin{tabular}{ll}
\hline 4. Flexible and opportunistic data & - Log of emerging constructs \\
& - Log of common definitions of constructs \\
collection method & - Reexamination of all cases for the \\
& emerging constructs
\end{tabular}

5. Within-case analysis and cross-case

- Summary of case studies

pattern search

- Table of similarities and differences for constructs

- Count of recurring details (Content Analysis)

6. Iterative tabulation of evidence

- Tabulation of the most frequent evidence

- Defining constructs based on evidence

7. Comparison with literature

- Tabulation of similarities and differences

8. Theoretical Saturation

- No more cases to examine

\subsubsection{Getting Started}

In case study research, the preliminary problem question is formulated and key variables identified using supportive evidence from the literature. At this stage it is important not to propose specific relationships between variables as this may bias and limit the findings.

Instead, the initial problem question is defined in the broadest terms to allow for the case study process to reveal the true specifics of the findings. 
Having reviewed the salient literature regarding living labs and comparing them to that of user innovation and co-creation; it was possible to extract similarities and differences between the three forms of innovation frameworks. The similarities and differences were then abstracted to formulate the "literature constructs" which laid the foundation to what may be, the main pillars of innovation platform.

\subsubsection{Selecting Cases}

Compared to traditional research methods, the case study approach is not reliant on statistical sampling and therefore does not require numerous cases or those selected to be randomly chosen. In the past, cases have been chosen to replicate previous cases of earlier research or chosen to fill theoretical categories to be tested. It was up to the discretion of the researcher to appropriately set the criteria of the case selection process.

The European Network of Living Labs (ENoLL) was chosen to be the source of data. This source proved to be the quickest and best-structured form to retrieve relevant information regarding each lab. Each living lab seeking to become a member of ENoLL is required to complete and submit an application form that is standardized with key questions.

1) Basic facts: Includes the name and structure of the organization as well as a description of the living labs operations, objectives and facts.

2) Membership Motivation: What the lab expects to gain from and what it will contribute to ENoLL. 
3) Description and Characteristics: The important capabilities and attributes that the Lab has and the defining characters that make them unique.

4) Organization: Describes the setup of the involved actors and stakeholders, their roles, contributions and experiences. This section also includes the governance structure and the extent to which the lab collaborates with other entities.

5) Openness: Addresses IPR regulations in the lab, degree of openness, membership process and the use of communication channels.

6) Resources: Sets out the required assets to operate the lab including equipment, funds and activities to operate the lab and means of acquiring such resources.

7) Users and Reality: How users are engaged within the lab and what measures it takes to create realism.

8) Value: Explains the different types of value (in the form of assets or other benefits) exchanged between the actors.

9) Direction and Future Plans: Portrays the metrics used for assessing success and what future objective the lab seeks. 
Because the cases need to be unbiased, the cases were not read prior to their selection. Instead, all the cases were available for selection including those from all possible countries. The cases were, however, limited between the time period of 2011 and 2012 to cap the amount of data examined.

\subsubsection{Crafting Instruments and Protocols}

The combination of multiple data types may help corroborate constructs and hypothesis making for stronger theory building. Case study research can therefore involve qualitative data only, quantitative data only or a mix of both. Choosing the data types also includes choosing the specifications for which the data must comply with.

This research was limited to the qualitative data extracted from ENoLL applications. The set specifications chosen for this data were that each case must have both application and lab profile complete. These specifications were used to help narrow the data set from three hundred and thirty-two cases down to forty-two.

\subsubsection{Entering the Field}

Good practice of case study research is the recording of field notes (Mack et al., 2005); the logs of ongoing commentaries about the researcher findings, both observational and analytical, during the collection and examination of the data. The field notes primarily give insight into what is being learned during the process and what similarities, 
differences and/or patterns have been developing. Based on findings recorded in the notes, the researcher may make adjustments to the data collection process such as adding more cases.

Procedurally, each application was examined with the intention of extracting information that fit within the defined constructs from the literature review. Application forms for each case were annotated with highlights that identify the information that fit within the set constructs. The highlighted data was then transferred to word documents and grouped according to their case. Chapter 4: Results, discusses the relevant outcomes of the changing thought process and lessons learned during the review of the application forms for each case.

\subsubsection{Analyzing Data}

The nature of the open-ended research problem question makes this step the most difficult (Eisenhardt, 1989). Analysis of the data is not necessarily well methodized because of the basic objective of seeking lessons from the case studies to help shape the constructs of the theory. Baxtar and Jack (2008) state that analysis framework 'should continue to develop and be completed as the study progresses'. It is important in this stage to avoid "analysis paralysis"; the over analyzing of data that resorts to conflicting results or a never-ending collection of data. Authors describing case study research, including Yin (2003) and Stake (1995), emphasis the importance of bounding the research (i.e. limiting the breadth and depth) in order to prevent analysis paralysis. 
The first stage of analyzing data was a detailed write-up of each case (within-case analysis) producing a pure description that summarizes important and relevant information. These descriptions provide insight into the field of research. To prevent the dangerous leap-to-conclusion from within-case analysis, the findings are coupled with cross-case analysis to identify or affirm patterns. The tactic is looking for within-group similarities and compares them with intergroup differences. The use of matrices to organize the derived inferences assisted with this tactic. Next was the measuring of the frequency of findings to determine the significance of the pattern and eliminating the outlying data.

The word documents containing the extracted data were converted into a summarized write-up that best described a living lab according to the constructs. Each write-up underwent filtering that eliminated information that was considered not important, repetitive and/or too specific for the purposes of describing a living lab. The case writeups provided a simplified overview of each case making it easy to do within-case analysis.

To measure patterns, content analysis was used on the case write-ups. The case write-ups represent the completion of data collection and a portion of the pre-editing process. The next step was to code the data for analysis (Tangpong, 2011). Weber's protocol was used for this as it offered a systematic way of coding and analyzing textual data. 
The first step was to establish a "recording unit". A recording unit is the measured unit and could be a word, phrase, theme or entire document (Tangpong, 2011); the unit must be meaningful to be effectively and reliably identified/measured (Krippendorff, 2004a). Words with multiple meanings can undermine the semantic validity of coding (Krippendorff, 2004a; Weber, 1990). To mitigate this issue, further manual pre-editing of the data was conducted to simplifying the sentence structures into singular context phrases and converted some words into clearly defined nouns. The chosen recording units for the cases were key words relating to the theme of the construct. The specific key words varied from construct to construct and are therefore mentioned in the Chapter 4: Results respectively.

The next step was to develop the coding rules that will be used to observe the units within the text. Coding is the technique by which one scans and measures the frequency of the word; it can be done manually via man-hours or through computers. Through the use of computer aids, large sets of data could be quickly analyzed while reducing erroneous problems related with human limitations (Krippendorff, 2004a; Morrison, 1991). Krippendorff (2004a) states, however, that computer aids should only help human coders in the analysis process rather than replacing them since human judgments and interpretation help legitimize the semantic measurements.

Therefore, the content analysis portion of this research underwent subjective analysis by myself; creating themes, sub categories and relations between common contexts. However, to codify the large amounts of data, MS Excel was used as the computer aid. 
Using excels built in OR, IF, ISNUMBER and SEARCH functions we constructed a coding formula in the form of a macro

$$
\text { [=OR(IF(ISNUMBER(SEARCH("KEYWORD", A2)), 1,0))] }
$$

The OR allows for the addition of key words, making various combinations in the single category search. IF returns true and thus identifies the phrase as being appropriate to the category, depending on the result of the search. As seen from the macro, the IF measures true for ISNUMBER, meaning being greater than 0 . Although a phrase may contain the key word multiple times, the purpose of the macro is to categorize the phrase and not measure the frequency of the words.

The macro was used to identify and group data points (i.e. phrases) based on the specified key word. Each key word combinations produced a grouping that further characterized the construct. A group termed OTHER was added to each search for two purposes: (1) highlight phrases that were not categorized, and (2) highlight phrases that were categorized multiple times. The code used for the OTHER category was the addition of all the categories. Excel equates a true to 1 and false to 0 . Data points that were not identified by the macro were discovered by the 0 that resulted from the adding of each macro-result. I manually reviewed data points that did not match keywords and placed them into the appropriate categories. Data points that we categorized multiple times were examined for the cause; often being a phrase that was not simplified enough. Using the count function, each subgroup was summed and compared to each other and graphed. 


\subsubsection{Shaping of Constructs}

At this stage, the emergent patterns and evidence from the case studies now help to refine the definition of the constructs. Note, not all cases may have all the measures nor alignment within the same measure making it impossible to have an encompassing definition. Therefore, it is important to establish validity for the definition of the construct through the tabulation of evidence.

Using the phrases that have been categorized into their respective themes and sub-groups, an explanation of what each construct is composed of and their practical description will further shape the Construct as a whole.

\subsubsection{Enfolding Literature}

This step is crucial to connecting the existing literature in the topic to the findings from the research. This step involves determining what is similar and conflicting and why such variances exist. By making the connections, readers are assured that the results of this research are correct and ties together underlying similarities in phenomena normally not associated with each other.

\subsubsection{Reaching Closure}


The finalization of case study research is marked by theoretical saturation (the point at which incremental learning is minimal) and the generation of concepts, conceptual framework or propositions. Unfortunately, case study research may disappoint by simply replicating prior theory or if no clear patterns emerged. Likewise, there are no generally accepted guidelines to assess whether the output of the research is "good theory" leaving the research subject to reader's discretion. 


\section{Chapter: Methods}

Chapter 4 describes the results of the research using the case study approach explained in Chapter 3 for the derivation and synthesis of the living lab constructs. This chapter is organized into four sections. Section 4.1 describes the initialization of the research, defining a general research question and establishing examination questions from the preliminary constructs from literature. Section 4.2 is the write-up results of the "withincase" analysis constituting a summarized version of the relevant information; this section is divided by case study. Section 4.3 describes the development of the emergent constructs for the production of the living lab model through the cross-case analysis process. Section 4.4 explains the shaping of the living lab model using the constructs and the testing of the model against other living lab cases for the purpose of replication and validation. Lastly, section 4.5 describes the outputs of the model by comparing it to the literature for conflicts and similarities and indicators the closure of the research.

\subsection{Literature Constructs}

To initialize the research, it was important to establish preliminary constructs that will guide the case study analysis. Table 3 shows the synthesis of emergent constructs from theory as discussed in Section 2.5. The constructs are shown in the first column and represent the commonalities observed between the three innovation streams. Therefore, representing fundamental constituents of the innovation concepts, the six constructs explicitly are: Stakeholders, Objectives, Governance, Tools, Motivation and Business 
Appropriation. The table compares the similarities and differences between the innovation systems as extracted from literature. The similarities and differences provide context for setting the scope and definitions of the constructs.

Using the synthesized constructs and their definition, questions were developed for each to be answered while conducting the within-case analysis and guide the review of each living lab. Table 5 summarizes these questions. The answers to the questions where noted into the logbook to help write up the case profiles in Section 4.2.

Table 5 Questions to Answer during Within-Case Analysis

\begin{tabular}{ll}
\hline Construct & Case Questions \\
\hline Stakeholders & - What organizations are members of the living lab? \\
& - What role do these stakeholders play? \\
& Who is involved in the different innovation phases? Idea \\
& Commercialization \\
\hline Objectives & - What does the living lab hope to achieve? \\
& - Why are they undertaking this project? \\
& innovation, their organization or their process? \\
\hline Governance & How do they manage the innovation process? \\
& - Who's involved in the management of the living lab? \\
& - Is there a team or a specific managerial structure? \\
\hline Tools & What resources do the living labs have at their disposal? \\
& - Do they use any specialized instruments? \\
& Testing? \\
\hline Motivation & Are the stakeholders intrinsically or extrinsically motivated? \\
& - What benefit do the stakeholders gain by participating? \\
& Does the lab have techniques to encourage participation? \\
& Reward systems? \\
\hline Business & How does the living lab make money? \\
Appropriation & - Who has control over the innovation output? IP? \\
\hline
\end{tabular}


complementary services?

\subsection{Case Study Profiles}

The profiles of the cases are presented in Appendix A. The cases have been arranged in alphabetical order for organization and search ease.

\subsection{Constructs Synthesized from Case Studies}

Following an analysis of the living lab cases through the use of the literature constructs as a guide, it was apparent that the emerging evidence required modification to the literature constructs. Table 6 shows the synthesis of emergent constructs from the case studies accompanied with the derived definition and scope.

Table 6 Emergent Constructs from Living Lab Cases

Case Definition $\quad$ Scope

\section{Constructs}

\begin{tabular}{lll}
\hline Objective & $\begin{array}{l}\text { The positive impact that the } \\
\text { innovation output is expected to } \\
\text { produce }\end{array}$ & $\begin{array}{l}\text { Collaboration, Social Impact, } \\
\text { Business Development, }\end{array}$ \\
& Economic Development, User \\
Impact, Test Bed, Framework
\end{tabular}




\begin{tabular}{lll}
\hline Case & Definition & Scope
\end{tabular}

\section{Constructs}

projects, process or organization are

made

\begin{tabular}{|c|c|c|}
\hline Philosophy & $\begin{array}{l}\text { Mindset of the organization that is } \\
\text { reflected in their level of openness } \\
\text { and collaboration }\end{array}$ & $\begin{array}{l}\text { Innovation Culture and } \\
\text { Intellectual Property Rights }\end{array}$ \\
\hline Stakeholders & Entities that add value to the living lab & Participants and their role \\
\hline Funding & $\begin{array}{l}\text { The means by which the living lab } \\
\text { financially supports its innovation } \\
\text { activities }\end{array}$ & $\begin{array}{l}\text { Public Funding, Private } \\
\text { Funding and Revenue Stream } \\
\text { of lab's Business model }\end{array}$ \\
\hline Advantages & $\begin{array}{l}\text { The benefits the stakeholders gain } \\
\text { from their membership and } \\
\text { participation within the living lab }\end{array}$ & $\begin{array}{l}\text { Product outcome, framework, } \\
\text { social value, business } \\
\text { development, validation, } \\
\text { resources, networking, } \\
\text { knowledge, investment and } \\
\text { marketing }\end{array}$ \\
\hline Communication & $\begin{array}{l}\text { The channels, technology and } \\
\text { techniques used to network } \\
\text { stakeholders for information exchange }\end{array}$ & $\begin{array}{l}\text { Online Presence, Media } \\
\text { Presence, Person-to-person } \\
\text { interaction, and more }\end{array}$ \\
\hline Infrastructure & $\begin{array}{l}\text { The necessary resources and } \\
\text { specialized equipment required to } \\
\text { carry out the innovation activities }\end{array}$ & $\begin{array}{l}\text { Software tools } \\
\text { Hardware, sensors, } \\
\text { Facilities }\end{array}$ \\
\hline
\end{tabular}




\section{Constructs}

$\begin{array}{lll}\text { Methodology } & \text { The procedural steps used for the } & \text { Attracting Participants, Ethics, } \\ \text { inception, development and } & \text { Motivation Rewards, User } \\ \text { deployment of innovation } & \text { Support, Data Collection, Idea } \\ & \text { generation, Design, Testing, } \\ & \text { and Commercialization }\end{array}$

Table 7, Presence of Constructs in cases, shows the number of cases that addressed issues regarding each construct. As seen below, not all cases contained the required information regarding the constructs. Of the forty cases analyzed, the least recurrent construct was Advantages having been mentioned within $75 \%$ of the cases. This does not imply that the construct was not discussed in detail but simply that of the forty cases, $25 \%$ of them did not mentioned advantages.

Table 7 Presences of Constructs in Cases

\begin{tabular}{lcc}
\hline Constructs & Number of Emergence & Percentage of Occurrence \\
\hline Objective & 40 & $100 \%$ \\
\hline Governance & 39 & $98 \%$ \\
\hline Philosophy & 37 & $93 \%$ \\
\hline Stakeholders & 38 & $95 \%$ \\
\hline Funding & 36 & $90 \%$
\end{tabular}




\begin{tabular}{lcc}
\hline Advantages & 30 & $75 \%$ \\
\hline Communication & 35 & $88 \%$ \\
\hline Infrastructure & 37 & $93 \%$ \\
\hline Methodology & 38 & $95 \%$ \\
& & \\
\hline
\end{tabular}

\subsubsection{Objective Construct}

Figure 1: Objectives of living labs, breaks down the occurrences, by percentage, of the various themes found to be the goals that living labs aim to achieve. It should be noted, that living labs could have several and concurrent goals but the Figure only measures the occurrence of goals relative to each other. The most popular objectives of the living labs in their respective order of highest occurrence are: Collaboration (24\%), Social Impact (23\%), Business Development (22\%), Economic Development (10\%), User Impact (9\%), ICT (9\%), Test Bed (1\%), and Framework (1\%). 


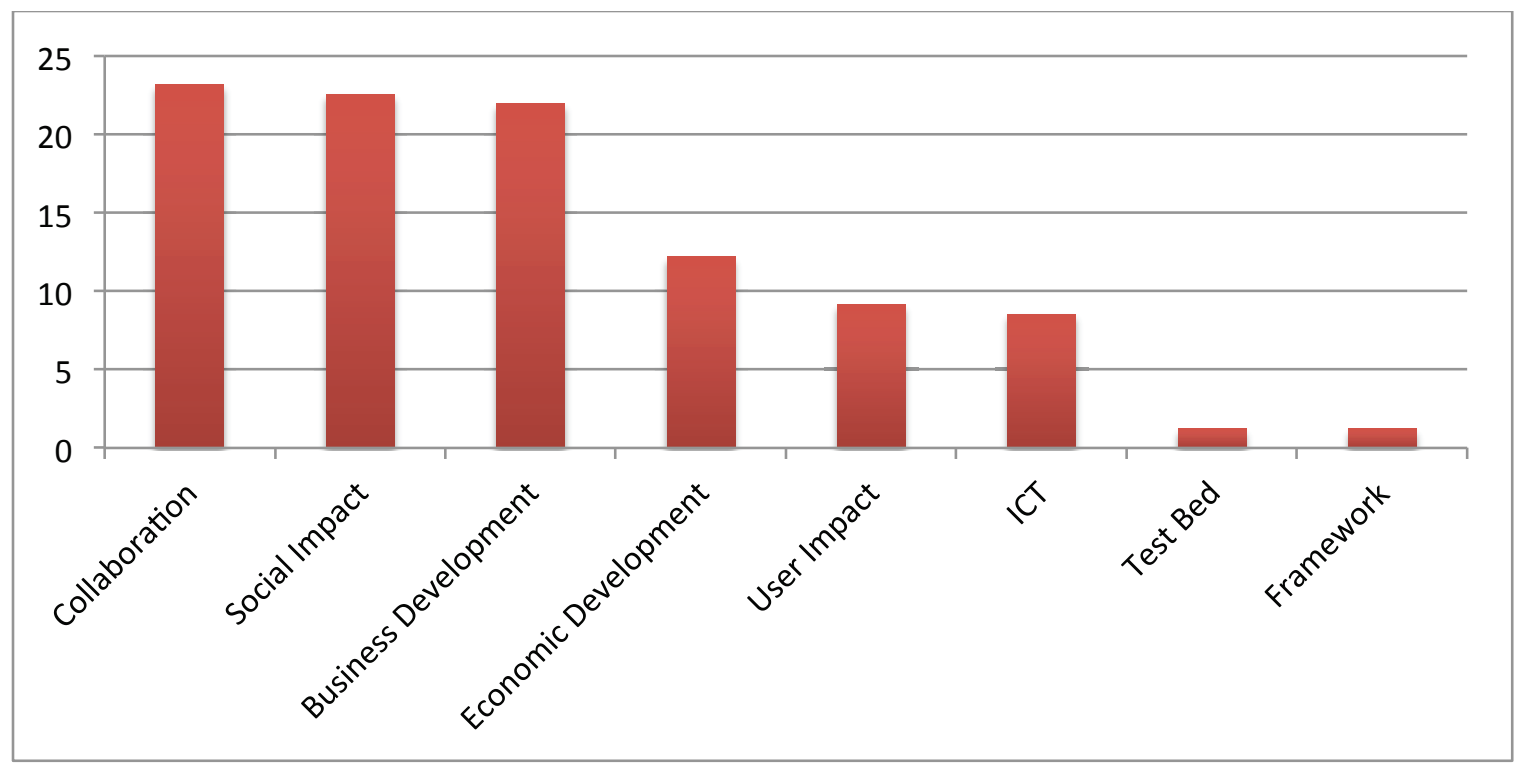

Figure 1 Observed and Measured Objectives of Living labs

To obtain these measurements, all case write-ups were analyzed regarding the objectives of the living labs, and relevant phrases were extracted into a word document. The phrases were subdivided based on the context of the phrase to singularize the information found in each phrase (ex. "Offers open configurable spaces" and "to build a better economy"). A total of 164 unique data points were generated. It was subjectively determined, by review of the phrases, that there were recurring themes, which were identified and termed: Collaboration, Social Impact, Business Development and Economic development. The remaining phrases that were not categorized, which accounted for 33 data points, underwent several iterations to define and place them within categories respective of their context. As such, four more categories were identified and termed as: User Impact, ICT, Test bed and Framework. Using the grouped data points, each theme is defined below. 
The collaboration theme appears to be the most popular objective of living labs with a $23 \%$ emergent rate from the objectives data points. This theme is defined as "the ambition to develop innovations by the communal effort of various actors". Labs make it a priority and goal to facilitate teamwork among the actors to share ideas, knowledge, best practices, experiences, resources and efforts for the purposes of developing novel products and services. According to the data in this theme, labs aims to establish joint operations to mutually manage such things as incubation space, state-of-the-art technology, knowledge databases and their users for the optimal creativity generation, cost-reduction and ecosystem network.

Social Impact was measured to have $23 \%$ emergence rate of the data relevant to the objectives of the lab. The theme is defined as "the aim to influence the community's environment and way of living". Based on the data, Social impact by living labs includes the urbanization of the region and the development of the regions infrastructures all without harming the biological ecosystem. More importantly, living labs seek to improve the quality of living for the regions citizens; improving the quality of air, their involvement in the community, and developing technologies that meet the citizens' needs.

$22 \%$ of the objective data was categorized under the Business Development theme. Business development theme is encompassing of all the goals the labs have for "establishing resources and services that support firms for the improvement and growth of their operations". Business development for the living labs includes product research, 
which comprises of activities such as needs discovery, product design, testing for validation and even commercialization. The complementary supportive services that the lab aims to offer are incubation space, market trend analysis, technology and education. Table 9, shows several more supportive goals as described in the data.

$12 \%$ of the objective data referred to economic development. This theme was determined to be "intent of investing in the region to drive employment, establish new business, foster competitiveness and accelerate entrepreneurship with lower barriers to entry". According to the data, this goal of living labs is to establish a sustainable economic growth in the region with fair rewards and distributions. In response to the ever-changing landscape of technology, this objective of the lab is to industrialize the region, bring innovations and competitiveness and attract more public to the region.

User impact theme is a category identified based on the focus of the objective; the focus being the end user. This theme was calculated to contain $9 \%$ of the data in regards to the objectives of the lab. As seen from Table 9, the data describes the lab's aim to output innovations that directly meet the needs of the users. The labs operations are geared towards customer satisfaction, designing their products in modular forms for interconnectivity, and working with users to deliver customized solutions. At the individual level, the lab aims to improve the way of living of each user by expanding the technological horizon, setting higher standards, offering better convenience, and produce technology that will help in their everyday lives. 
$9 \%$ of the data focused on the living lab's objectives to develop Information Communication Technology. As seen from Table 9, the development of ICT is not limited to the establishment of telecommunication infrastructure, but rather, developing, testing and commercializing ICT products such as mobile products to help expand the regions digital position. These labs work towards raising the awareness of the importance of ICT and pushes for the upgrade to the latest and newest ICT.

Lastly the last two themes, which each had $1 \%$ of the data, are the establishment of test beds and the development of methodological frameworks. Living labs also aim for establishing a realistic environment (test bed) for testing and validating products, and creating an actual methodological process (framework) for members to follow during the user-centered product development cycle.

\subsubsection{Governance Construct}

Figure 2: Legal Structures, breaks down the occurrences, by percentage, of the various legal forms a living lab could take. The most legal form of living labs in their respective order of highest occurrence are: Private (31\%), Public-Private Partnership (19\%), Publicprivate-people partnership (13\%), Public (13\%), and undefined User Impact (25\%). 


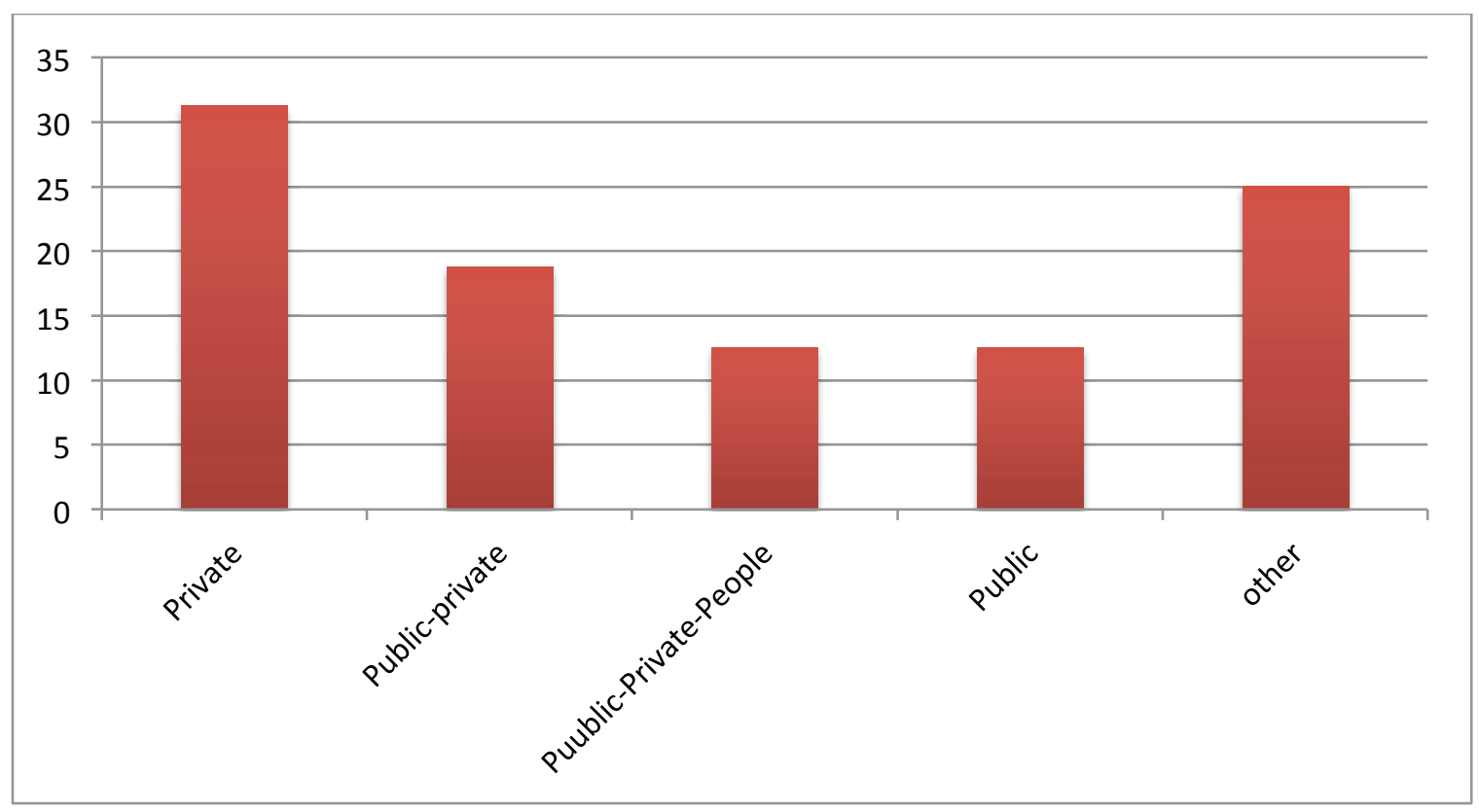

Figure 2 Observed and Measured Legal Structures of Living labs

Several attempts were made to use content analysis on the decision making structure of living labs, however no measurable patterns were sufficiently identified. Through a rigorous review of the cases, it became apparent that the data pointed to each living lab having its own decision making process. However, interpretively analyzing the semantic contexts of the descriptions, it is apparent that there exist managerial structures that mimic a top-down governance structure. These managerial structures often consist of the founding members of the lab, or representatives for each group of participating members. It could therefore be interpreted to imply that the top-down governance incorporates a bottom-up approach.

An example of a structure some living labs use is an Executive level management team in conjunction with an advisory board. The executive level makes strategic decisions for the living lab; they set goals, choose which projects to undertake, how to allocate resources 
and negotiating relevant organizational requirements (e.g., how to accept members). The advisory board is a team that consists of field experts (e.g. scientists, market gurus). They help to analyze existing knowledge and make recommendations to the executive team that helps guide their decisions. Regardless of the governance structure of the living lab, there are several responsibilities the management teams take on.

The governance group of the living lab is responsible the overall activity ensuring that the activities meet the goals and objectives. They are the leaders who take on the administrative and managerial work. They set the vision and make investment decisions, manage IPR, engage stakeholders and review application for support. Applications for funding are done by the governance group because they monitor the performance of the lab, organize its activities and review all communications and reports. Without them, changes to work packages, procedures and resource budgeting when be chaotic and are therefore given authority to make the necessary decisions including the resolution of intergroup problems. The governing group is self-regulating as they are in control of approving new members, appointing coordinators and establishing networks to attract further sponsors and partnerships.

The governing group is also responsible for the project level decisions. They are responsible for the technical and economic aspects of the projects developments. They not only select the projects to pursue, but assign the appropriate members to oversee and run its activities. Duties include maintenance of the labs infrastructure and the engagement of the user test panel. Research planning process is mandated in their job 
description, as they are responsible for the methodologies to be executed and the setting up of user-centric approaches.

\subsubsection{Philosophy Construct}

Figure 3, Philosophy Categories, shows the primary weighting of the two themes identified within the philosophy construct. It is seen that the data found within the Philosophy construct addressed more issues regarding the handling of Intellectual Property Rights (IPR) in comparison to the innovation culture of the living lab. As seen from the Figure, $58 \%$ of the collected data on the labs philosophies give insight into IPR issues over the $42 \%$ discussing the attitudes and perspectives of the lab's innovation culture.

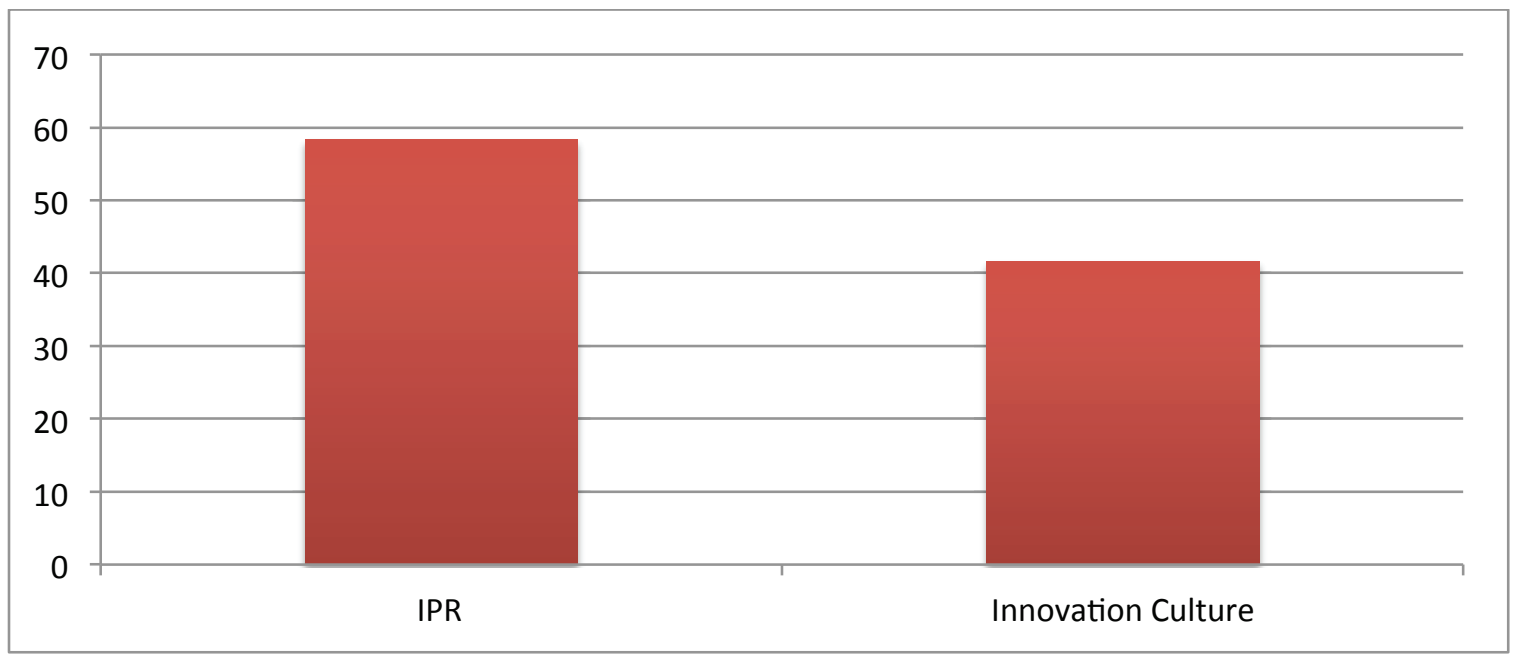

Figure 3 Observed and Measured Philosophy Categories for Living labs

The data for this category was obtained from the case write-ups based on analysis regarding the philosophy matters of the living labs. The data was extracted into a word 
document where they were further divided into sub-points based on the context of the phrase (ex. "Open to new members" and "uses open source licenses"). The sub-points that explicitly mentioned IPR matters were grouped together into the IPR category while the remainder was grouped under Innovation Culture. The two categories were compared together in excel to generate Figure 3 by counting the number of sub-points generated relative to the total data points extracted. Each category then underwent text analysis as explained in Chapter 3 section 3.4.5 using various key words that stood out during the initial read.

\subsubsection{IPR Management}

Figure 4, IPR Management, summarizes the various categories the data of IPR issues were further grouped. The categories represent the identified methods used by the living labs in managing IPR related issues. The identified methods categories are: Consortium Agreement (33\%), OEM (14\%), Licenses (12\%), Open-source (12\%), Case-by-case (10\%), Law (8\%) and Other (11\%). 


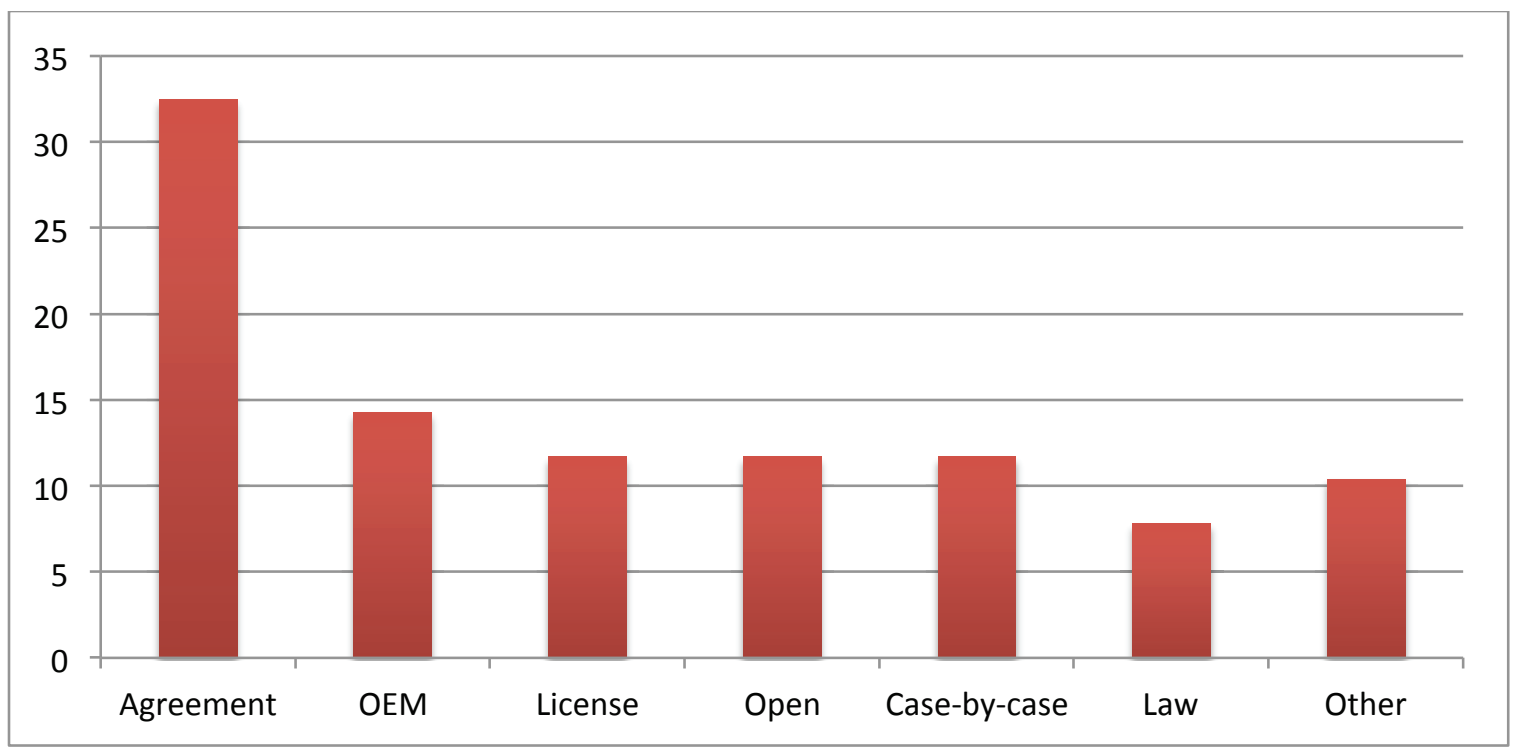

Figure 4 Observed and Measured IPR Management Techniques used by Living Labs

A subjective review of the data produced the mentioned categories. Using the technique mentioned in Section 3.4.5, the data was grouped using the categories title as key words; respectively this translates into: Agreement, Original Owner and the Original Equipment Manufacturer (or OEM), License, Open Source Licenses, Individual (or Case-by-case), and Law. After the first iteration, it was noticed by the OTHER column, that some phrases were related to the identified themes but did not contain the key words. Therefore, it was manually placed into the appropriate themes. The phrases that could not be placed were then analyzed for their most common words and context but could not be grouped into new categories.

A significant portion of the data in the IPR management set was grouped into the Consortium agreement category. Compiling the data together, it appears that living labs will set forth rules and regulation regarding the use, sharing and licensing of IP prior to 
the initiation of a project. These set of rules are formulated and agreed upon at the organizational level and is required to be signed by all members who wish to participate. Using the framework set out by the lab, the agreement grants specific rights for the use and exploitation of the knowledge and outputs the lab generates. The lab signs its members to confidentiality agreements to protect sensitive information regarding IP or personal data. The agreements can also outline the distribution of cost and gains for each member depending on their role and investment in the developments. The agreement is the lab's technique to regulate IP under common and agreeable terms and ensuring security for the participating members.

$14 \%$ of the data in this set discusses the assignment of IP rights to the originator of the output, the OEM. The data describes this approach, as a promise ensuring that all IP brought into the lab remains the sole property of the originator and the degree to which it is shared, licensed, and controlled are under the discretion of the originator. In like manner, any output produced is the rightful property of the creator. In collaborative work, the individual modules, or leader of specific components is considered the creator of such part.

Third largest data category addresses the use of Licenses for the handling of IP issues. The data points to the notion that licensing in this situation could be directly from the OEM or from the lab itself. Unlike the previous method, the data appears to suggest that labs in this scenario will mandate from its participants two conditions: (1) that members must provide licenses to improve or develop using the IP and, (2) license to 
commercialize the old and new IP. This method ensures benefits to other members in accessing and utilizing each other's IP. Although it is mandated, the licenses are mutual between all members and agreed to under fair market conditions.

The open-source category was found to have equal amount of data as the licensing category. As seen from Table 10, the use of open-source is comparable to regular licensing agreement, wherein, it maintains that all outputs, and possibly original IPs, be subject to open-source standards. This entails that all material in the living lab is available for modification and distribution under the specified open-source license (often GNU). This method of handling IP maintains openness within the lab and sets freedom to all members to benefit from the innovations.

According to the data, to respect member's contributions, position and intent, living labs can also handle IPR issues on case-by-case bases. Prior to the commencement of a project, the participating members must agree to a fair distribution of rewards and rights based the resources and amount of effort each will invest. This method excludes the living lab and leaves the terms and conditions to be developed and agreed upon by participants. For this reason, the lab does not guarantee any rights or protection regarding IP to any of its members.

The last identifiable method of handling IPR issues, and comprised of $8 \%$ of the data, was the compliance of the regions Law. This method simply states that all IP issues 
involving the lab will follow standard laws and did not specify any special practices by which the lab will respect, control or govern the IP it uses or outputs.

The unidentified data, represented by the OTHER bar is composed of various methods that do not appear to have a relation to other methods or does not have reoccurring context. One data point institutes that all outputs are the sole property of the municipality and to be exploited only by them. Another data point has set that all IP issues be handled and dealt with by the University. Similarly, other data lets Technology transfer organizations handle the IP issues on behalf of the lab.

It was unclear from the data weather or not there is crossover in IPR methodology.

\subsubsection{Cultural Topics}

Figure 5, Cultural Topics, breaks down the different themes that the cases address regarding the innovation culture within living labs. The number one topic, that retains $53 \%$ of the data points from this set, is the theme of Collective Effort among the actors. Next to follow with each having $15 \%$ of the data points are the themes of Accepting Members and the use of Open Source material. With $7 \%$ of the data points, the topic of the consortium agreements that dictate the member's code of behavior is the fourth topic. Lastly, $4 \%$ of the data in cultural topics were regarding the Open Thinking of the living labs and how they need to be open-minded when collaborating together. 


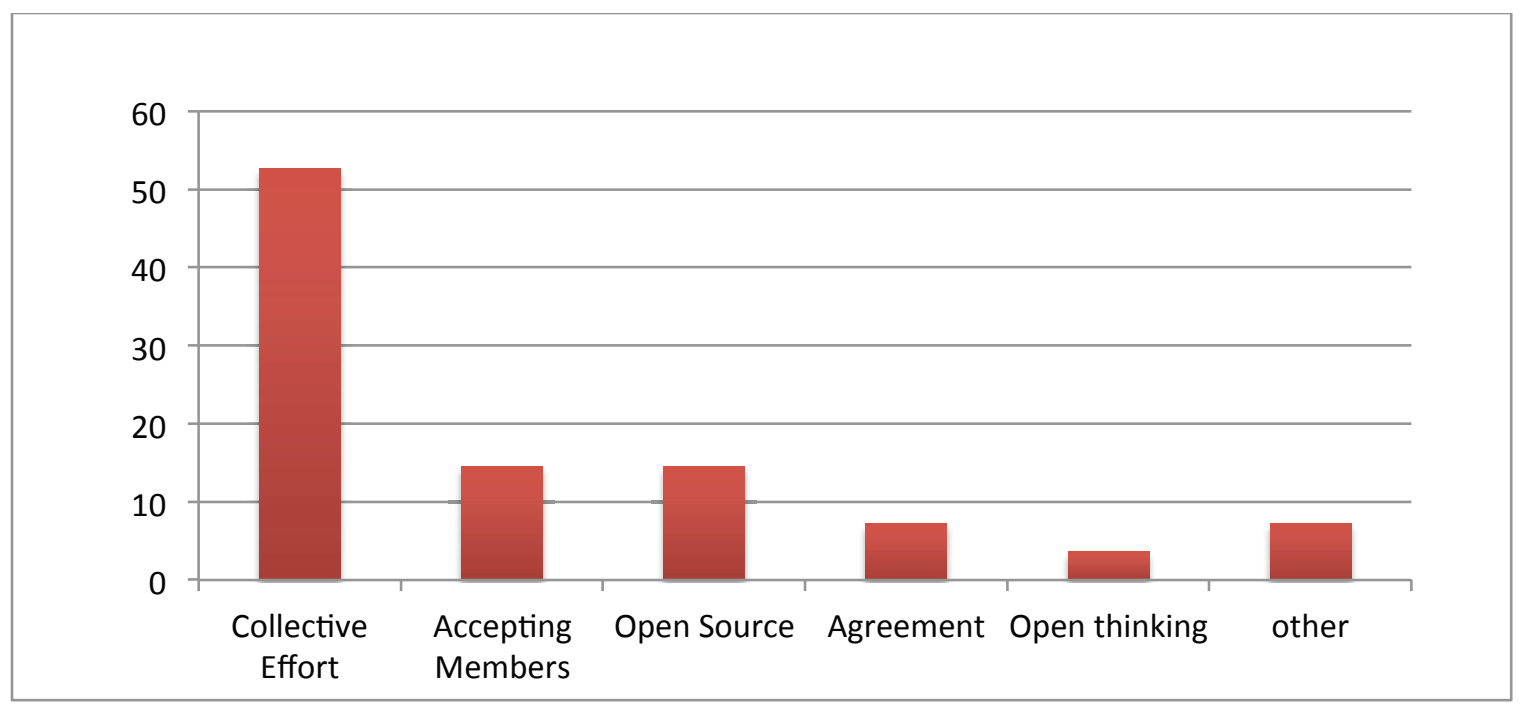

Figure 5 Observed and Measured Innovation Culture Topics

After a personal review of the points in the innovation culture, it was decided that there were three recurring topics; Collective Intelligence and Effort, the Acceptance of new Members and the use of Open Source. The method mentioned in Section 3.4.5 was used to categorize the data into the mentioned topics. After the first iteration, it was noticed by the OTHER column, that some phrases were related to the identified topics but had not contained the key words. I therefore manually read and placed, those phrases into the appropriate topic categories. The phrases that were not categorized were then analyzed for their most common words and context and produced an additional two themes: Consortium Agreement and Open Thinking. However, there still remained, a set of phrases that did not share significant commonalities.

The key words used to identify phrases for the collective intelligence were; openness, intelligence, team, and collaboration. Excel categorized 29 of the 55 data points $(53 \%)$, into this topic. Summarizing the data points, this topic describes the value of teamwork in 
living labs. Based on the data, there is an important mentality in the labs to encourage and promote collaborative work among the members for the purposes of achieving innovation, entrepreneurship and their other goals. As seen from the data found in Table 11, the labs maintain that members must respect each other and participate in the ecosystem of sharing knowledge. The labs therefor develop a framework that tries to reduce barriers as much as possible that impede the cooperation of the members. They grant access to the labs knowledge, use open standards to enable them to use free tools and push for co-creation process.

The key words used to identify the phrases for 'accepting new members' category were: accept, open to, and welcome. This category discusses the degrees to which labs accept new members. As seen from the data, found in Table 11, labs are very open to accepting new members because it creates diversity and adds value to the lab. It was found that, while the majority of the data supports that labs are open to accepting any member who wishes to participate, some limited their selection to those who need help or those that can contribute to the labs activities.

To categorize the data points into the Open Source topic, open-source, use of, utilize, and free software were used as key words. The grouped data revealed that open-source had an equal emergence rate as membership in regards to the topic of innovation culture of $15 \%$. Examining the data yielded a notion that living labs use open-source standards to help with the integration of participant's offerings. According to the data, the use of opensource standards allows for the offering of free tools to help enable members to co-create. 
Most importantly, labs will use open-source standards to avoid IP issues and work with an open framework that promotes collaboration for innovation.

The topic agreements were synthesized from the data based on data that was grouped together using the key word "agreement". With 7\% of the data, this topic discussed the use of consortium agreements for the purpose of outlining the conduct of the labs activities and members. It includes how they will accept new members, the confidentiality between the participants and the operations of the members.

The last topic that was identified was open thinking and was identified by the key word "unlearn". Although it had few data points, it presented an important fact that living labs aim to achieve for their innovation culture. Living labs must try to unlearn old paradigms of individual thinking and hierarchical management techniques that impede the creative energy of open collaboration.

\subsubsection{Stakeholder Construct}

The data regarding living lab stakeholders could not be efficiently analyzed due to the type of data collected. Although there is significant data regarding the involved stakeholders, it is not properly formatted for correct codifying making it significantly difficult to content analyze for patterns. What could be identified from subjective review of the cases is diverse involvement of companies, unions, universities, governments and civic organization/associations. 
More importantly, it was found from the data that there is a win-win relationship between the involved stakeholders and the living lab as a whole. All the resources utilized by the lab are pooled in part from each member. Stakeholders that cannot contribute physical resources are obligated to contribute in other forms such as funding, invested effort, innovations, and/or knowledge. In this manner, the living lab can be viewed as a private good, where no participating member is simply uses the lab without returning the favor in one way or another.

\subsubsection{Funding Construct}

Figure 6, Fund Sources, shows the three sources of funding that living labs have at their disposal in their respective weight of based on data points per source. Government funding was mentioned the most and has $40 \%$, while private funding and the lab's revenue stream are equal both are split at $30 \%$.

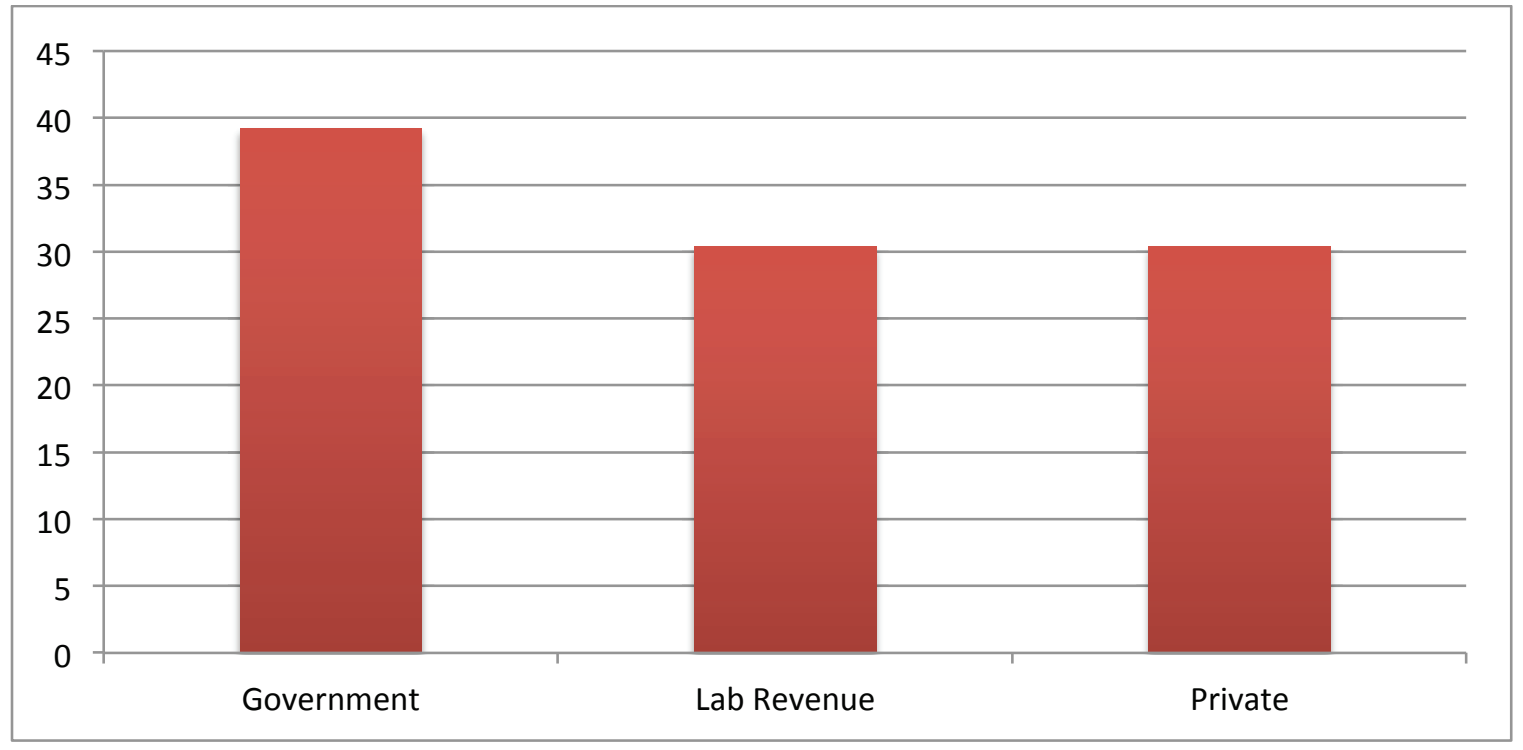




\section{Figure 6 Observed and Measured Sources of Financial Support}

The data collected from the cases-write ups were divided into separate and unique data points and categorized into three categories, Government Funding, Private funding and Revenue Stream. The categorization process was done through excel using the method outlined in section 3.4.5. The keywords used to isolate the phrases regarding government funding were: public, government, national, international, local, and grants. In comparison, to differentiate the private funding, the following key words were used: private, members, partners, industry, and sponsorship. The remaining phrases were categorized into revenue stream and confirmed by a simple review. If key words were to be used to identify this category, they would include: revenue, business model, services, independently, and sustain. Have been segregated, each category's points were summed and compared to the total points of data.

Government funding as classified from the data, comes from local, national and international sources. These funds can be offered by the government through various organizations and programs that aim at driving innovation, building the economy, or intent on making a social impact. Similarly, the funding that is received from international groups comes from European Union Programs that help support research and employment. Private funding comes from participating actors of the living lab.

According to the data that can be found in Table 12, funding is used primarily for the purposes of offsetting the cost of the lab's operations and compensate for equipment. The funding can also be used to offer its members a cost-effective (i.e. subsidized) way of 
developing innovations. As seen from the data, funding has also been used for marketing purposes; driving the commercialization of the labs outputs, calling for proposals, and raising awareness. In the case of revenue stream, the money gain is used as a metric to measure the success of the labs follows through.

\subsubsection{Sources of Revenue}

Figure 7, Sources of Revenue, shows the breakdown of the various revenue streams of the lab's operations. As seen from the Figure, much of the data (56\%) supports the lab's major source of revenue being consulting. The next largest revenue stream is from royalties $(24 \%)$. Living labs also make money from rent $(12 \%)$, membership fees $(6 \%)$ and educational programs (3\%).

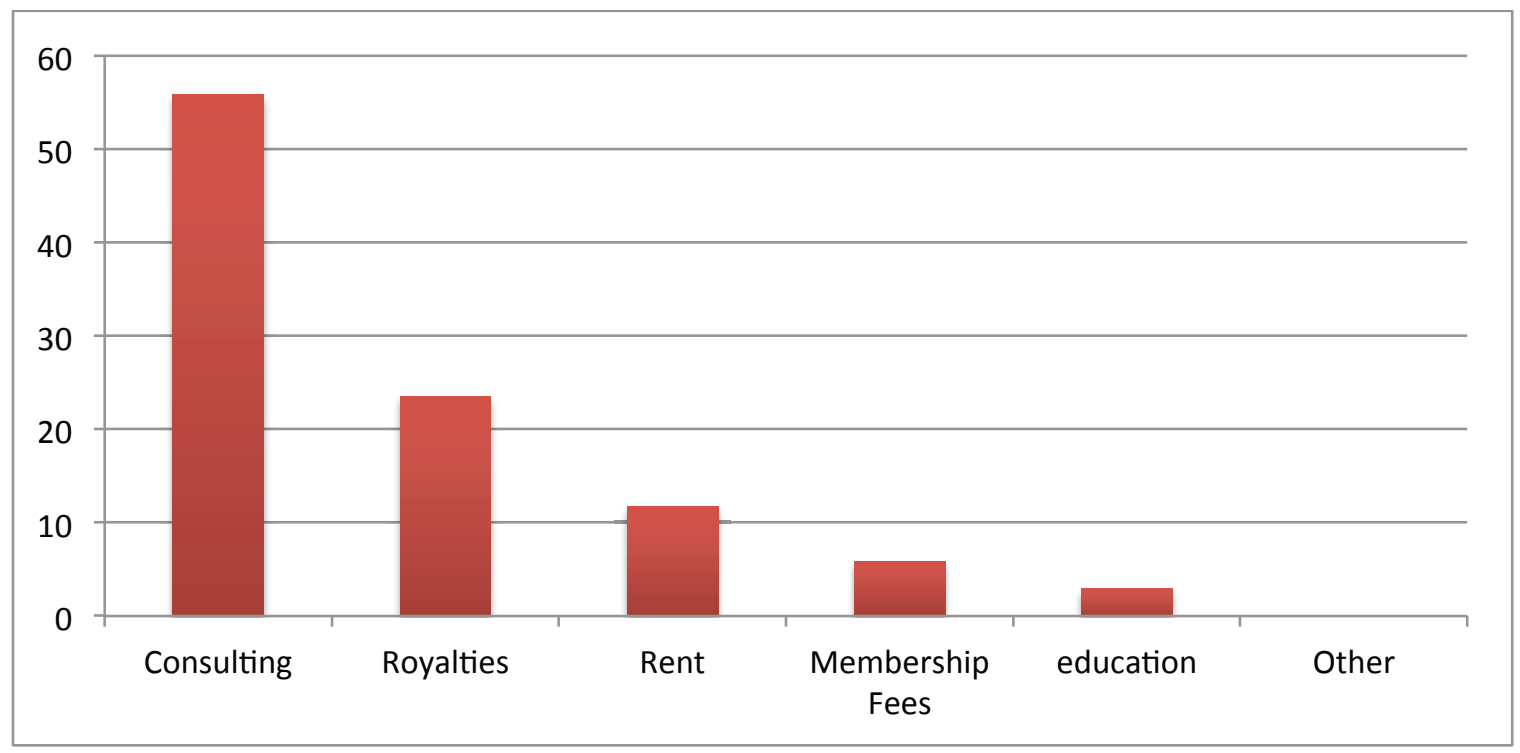

Figure 7 Observed and Measured Streams of Revenue forming their Business Model 
Using the data points categorized under the labs revenue stream, I ran a text analysis using the method mentioned in section 3.4.5. The key words used to identify the consulting phrases were: service, support activities, consulting, and maintenance. Keywords used to group phrases regarding royalties were: royal, sale and output. Rent and membership and education streams were their own key words. Using the other column, any phrases that were not identified were examined and manually placed into a stream.

The data points for this stream or summarized in Table 12. As seen from the table, Consulting is a broad and an encompassing revenue stream and is comprised of the many services that the living lab offers to its members and third parties. It short, consulting revenue stream is when the living lab receives payment for services rendered to business, government or consumers. Because each lab has a different focus, the services offered can vary and therefore were all grouped together under the umbrella of consulting. Some forms of consulting that living labs offer are: digital marketing, data collection and analytics, training, product evaluations and more.

Royalties is the second revenue stream identified from the data. It was found that labs would produce income from the innovations it outputs in the form royalties and/or sales. This form of revenue income structure does not strain the members financially; instead, only makes profit when its members succeed in the commercialization of the products. 
Another form of income for the lab comes from the leasing of its resources to third parties. The data shows that labs can rent to others the facilities for event purposes or lab research and also rent out the equipment they possess or create. An example is the renting of the 3D reality (virtual city) to simulate product requirements.

The data shows that Living labs also have the option of generating income from its partners through a membership fee. Membership fees guarantees commitment to the lab and a form of financial support independent of private investment.

Lastly, labs can also offer educational programs that help support and advance its members. Although knowledge sharing is often free within the lab, instructive curriculums can become revenue generating when offered at a cost.

\subsubsection{Advantages Construct}

Figure 8, Advantages, graphs the various benefits the lab offers to its members in order of their most occurrences in the cases. Ten different benefits have been identified from the data: Business Development (17\%), Knowledge (16\%), Resources (11\%), Networking (11\%), Validation (10\%), Marketing (8\%), Product Outcome (8\%), Framework (8\%), Social Value (7\%), and Investment (5\%). 


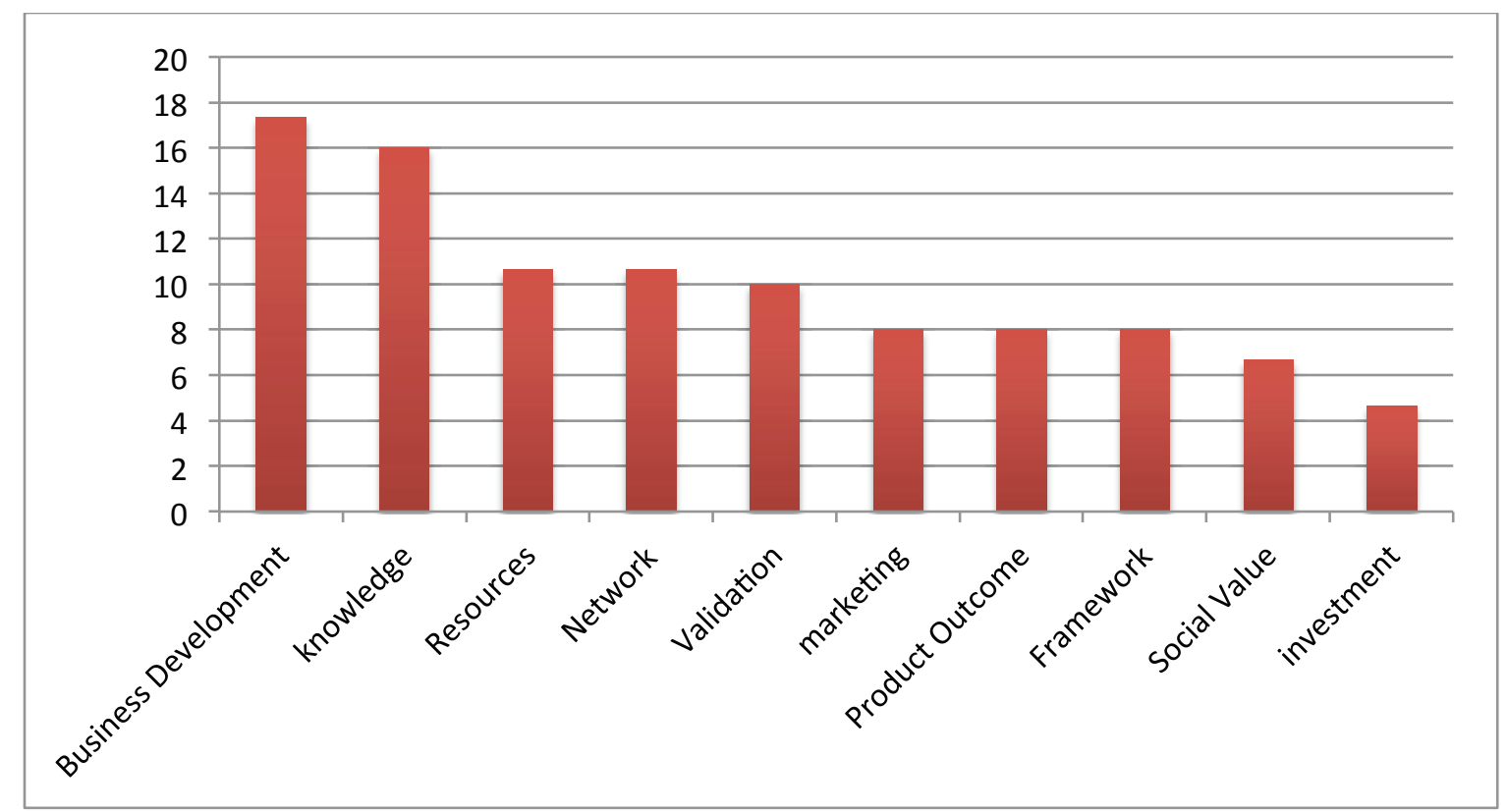

Figure 8 Observed and Measured Advantages Living Labs Provides to its Members

All case write-ups were analyzed regarding the advantages of the living labs, and extracted into a word document. The points were further divided into sub-points based on the context of the phrase (ex. "legitimize members" and "network with other business"). A total of 150 data points were generated. Prior to their input into excel, it was subjectively determined that there were recurring benefits, which were: Business Development, Knowledge, Resources, Networking, Validation, Marketing, Product Outcome, Frameworks, Social Value, and Investment. The phrases were then inputted into excel for text analysis as explained in Chapter 3 section 3.4.5, using key words of the identified benefits. After the first iteration, it was noticed by the OTHER column, that some phrases were related to the identified benefits but did not contain the key words. I therefore manually read and placed, those phrases into the appropriate benefit groups. 
Of the 150 data points, 26 points were categorized as Business development. Business development is defined as the "supportive activities that aid the members to achieving their business goals". According to the data that is summarized in Table 13, business development includes services such as management support, advisory teams; consultation professionals that are offered to support the members. It encompasses various supportive activities such as design, expansion, digital marketing, technology transfer, project development, and personal growth that directly or indirectly help with the growth of the business.

24 data points of the 150 were categorized as Knowledge related benefits. As seen from the data (Table 13), members join living labs to tap into the wealth of knowledge it provides through its own activities and the activities of its other members. The lab is shown to be a facilitator of knowledge exchange, conducting research and development with the help of its members in various fields such as health, ergonomics, ICT, art and more. Through proper analysis, much of the data generated can provide many answers to the members. The data suggests that access to this data is offered to the lab's members in exchange for their participation. Member's experiences and expertise have also been counted as knowledge, specifically a form that is difficult to obtain. The Lab continues to extend knowledge to its members through training, courses and workshops.

With $11 \%$ of the data points, Resources score third highest as a key advantage for members. Technology platforms including lab facilities, research facilities, knowledge sharing facilities and more are offered to help enable members to conduct research and 
development. The data also mentions, incubation space, state-of-the-art technology, test beds and free access to content already generated by the lab are also provided.

One of the identified advantages to being a member of a living lab is the opportunity to network with other members. $11 \%$ of the data mentions networking. Because the lab attracts a diverse group of participants, networking within the living lab not only expands ones horizons in their own industry, but also makes connections with new industries. As mentioned by the data, once networked, members gain access to new markets, form partnerships, make strategic alliances and collaborate together for effective success.

Part of the data showed Validation of products and services prior to commercialization is also an advantage. The 15 points explain how living labs help members test and evaluate the produced innovations through users in a realistic environment. The testing process living labs use helps accelerate the development of the product (i.e. design feedback) and also establish an initial market presence.

The data revealed that one of the advantages of joining a living lab is the development of one's market position. As a member, one can receive visibility for your brand in addition to the legitimacy gained from participating in collaboration. Logos are included in the labs advertisement campaigns and the member's works are showcased during their events. A business member can use the labs access to users and partners to tap into new market segments to provide their offerings or complement existing products. 
Product Outcome is a generalized title for the data that describes various benefits directed towards an improved product. The benefits mentioned by the data for products range from acceleration of production, to improved quality and lower cost. Other benefits include modular designs, customized solutions, complementary technology, and reduced risk of investment and market launch.

The methodological framework living labs operate appears to have the same amount of support from the data as does the marketing advantage. Based on the 12 data points in this category, Living labs offer to their members a structured process that enables collaborative work. Specifically, the framework can be tailored to find niche markets, benchmark data, sets evaluation metrics and produces sustainable business models.

One of the identified advantages was termed social value. In simplest terms, social value is the benefits gained as a society in whole. Such benefits, as seen in Table 13, include economic growth, job creation, open culture, and a sense of pride for the region.

Lastly, the data shows that living labs also provide financial aid to its members. With access to government and private funding, the lab can allocate money to personal development, product research, or any necessary activity need to secure success for its members. The lab itself can help its members apply for funding from various organizations.

\subsubsection{Communication Construct}


Figure 9, Modes of Communication, displays the three main forms of communication and the amount of data that supports each. As seen from the Figure, the majority of the data supports online mode of communication with $40 \%$ data points from the set. The next most common mode of communication is through the use of media channels with $30 \%$ of the data. Lastly, physical presence (e.g. events) has $26 \%$ of the data. There was $4 \%$ of the data regarding communication that could not be categorized into a specific mode of communication.

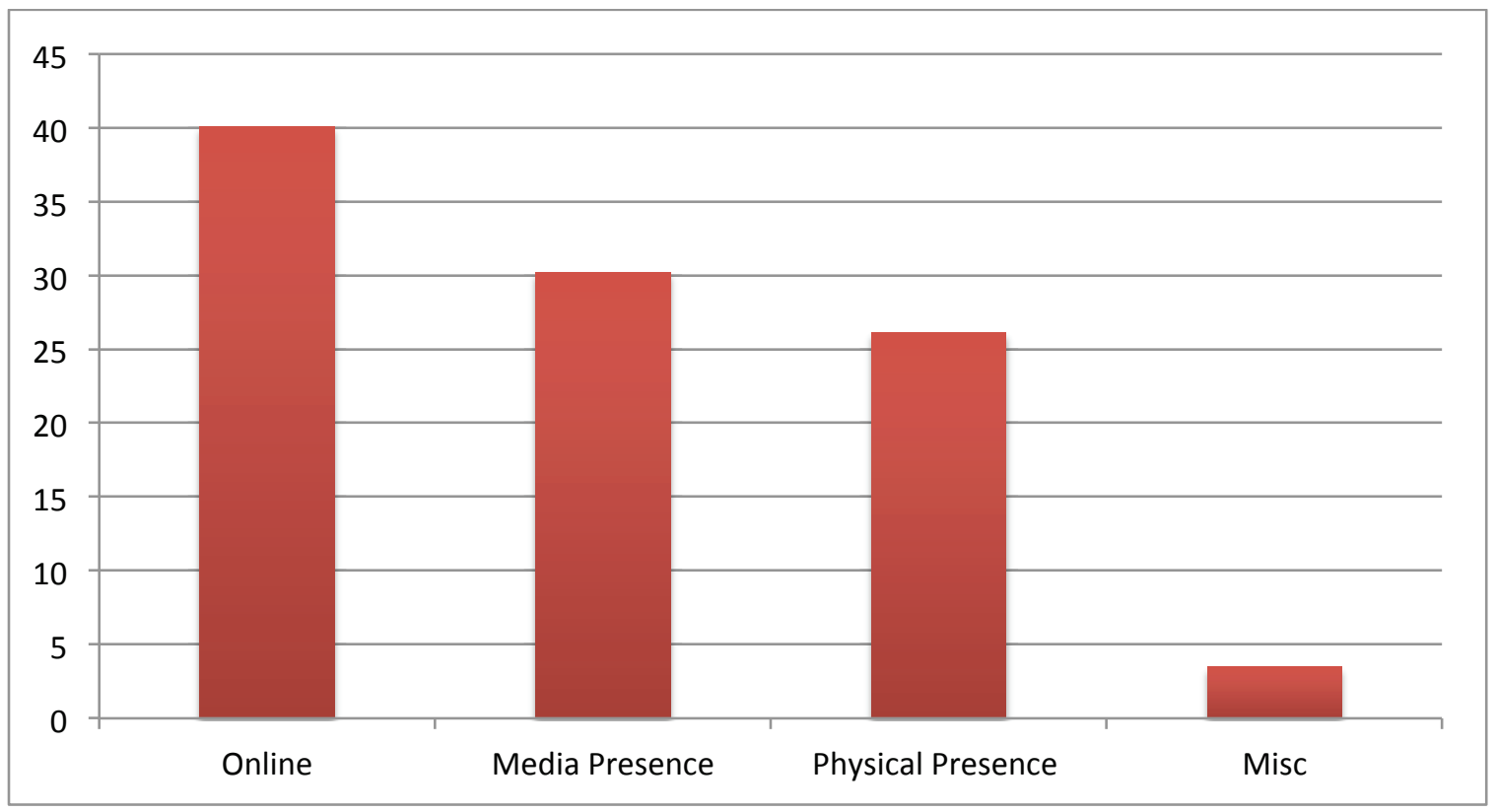

Figure 9 Observed and Measured Modes of Communication

This data was formulated from the examination of the case studies write-ups for all relevant information regarding communication efforts. The data was placed into a word document were the data was subdivided into various points based on the contextual value of the phrase. The 172 different points generated were then categorized into three groups 
based on their main subject: Online, Media, and In-Person. The points that did not have a clear subject orientation were grouped together and termed Miscellaneous. Using Excel, the number of data points were counted for each group and plotted against each other by percentage of total set.

The data grouped in the online category encompasses all relevant communication channels that require an Internet connection or database server. This category includes websites, social media, virtual environments, and interactive tools such as forums and blogs. Media Presence is the second category that includes channels such as television, newspapers, magazines and more. Although the media can have an online presence, this category has been distinguished from online due to the fact that they are not dependent on the Internet and can have physical forms of communications. The media category is geared towards the use of mass communication mediums controlled by other organizations. Lastly, the third group is the Physical presence as defined by the need to have an assembly of persons in the same location and free communication independent of technology.

\subsubsection{Online Communication}

Figure 10, Online Communication Channels, shows the different channels a living lab utilizes as part of its online mode of communication. As evident from the bar graph, the online channel with the most data referencing it (49\%) is a customized website. The next most common channel of communication through online means, as measured by the data 
count $(31 \%)$, is social media. Compared to the two major channels, the other online channels living labs have been identified to use are: Virtual presence $(6 \%)$, blogs $(4 \%)$, forums (3\%) and mobile platforms (3\%). Lastly, 3\% of the data was found to be online related could not be categorized under any specified channel.

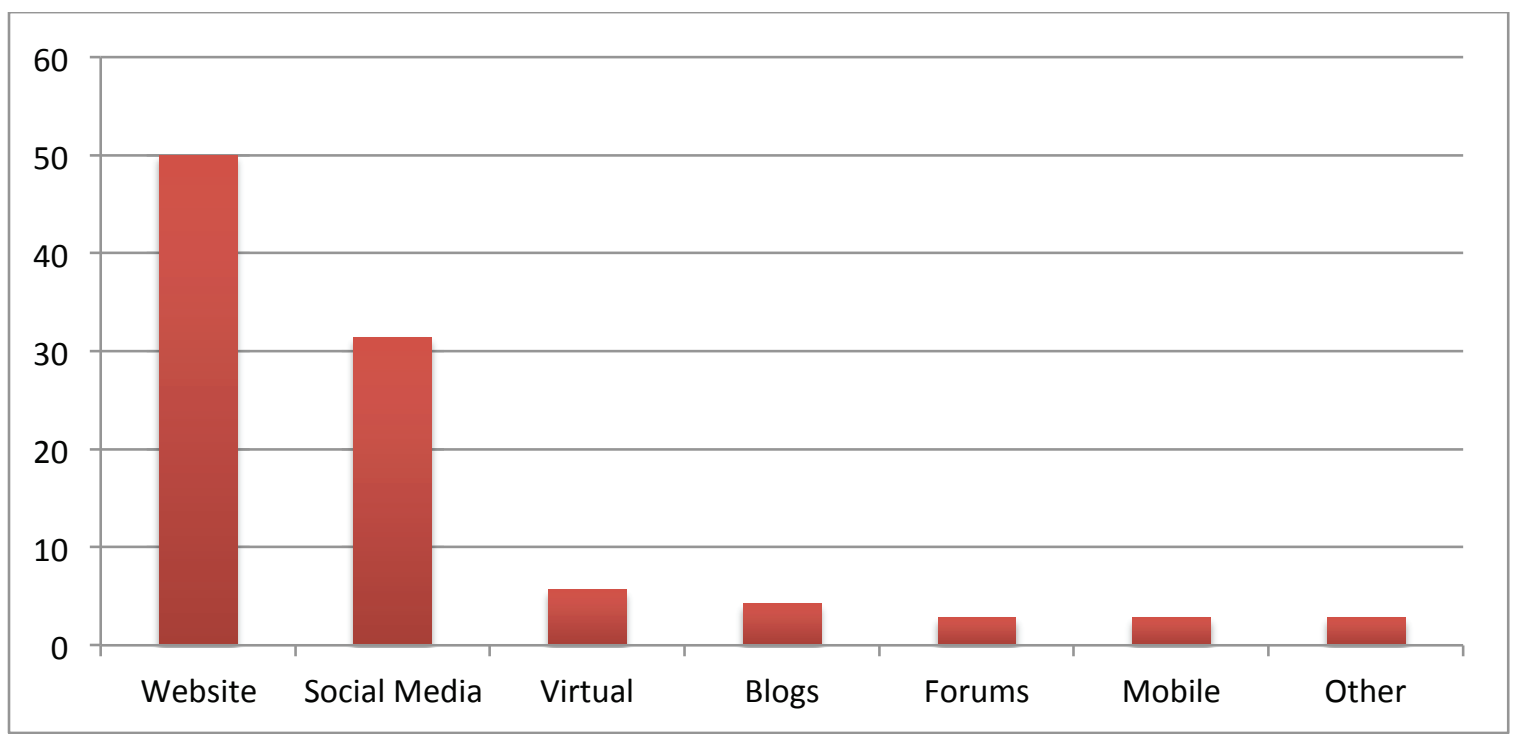

Figure 10 Breakdown of Online Communication Channels Used by Living Labs

Of the 172 data points identified for the communication construct, 69 phrases were categorized as a form of online channel. Using the method in chapter 3 section 3.4 .5 , the data was grouped into two main camps, Websites and Social media. The key words used to place the phrases into the website category were: website, web portal, portal, and site. The social media category was identified by a search of key words such as: social media, social networks, Facebook, YouTube, Twitter, RSS and Slideshare. Of the 69 data points, 56 points was automatically identified into of those two main channels. The remaining 12 points were examined manually for possible repetitive themes. It was decided, based on the recurring key words, that four more channels exist for online communication, these 
are: Virtual environments (key words: video, live, virtual), Blogs (key word: blog), Forums (key word: forum) and mobile platform (key word: mobile).

The various channels of online communication are self-explanatory. However, it should be noted that the website data did include the use of both a personal site (belong to living labs) and member sites (pages hosted onto a member owned website). Additionally, the virtual environments channel encompasses two different things. One, the virtual channel includes the use of video conferencing such as Skype and two, the use of simulated virtual realities such as SIMS lab.

\subsubsection{Media Channels}

Figure 11, Media Channels, shows the six channels of communication using media outlets. The TV outlet had $23 \%$ of the data points, the press $19 \%$, publications $17 \%$, Advertisements $15 \%$, radio $15 \%$, and magazines $8 \%$. $2 \%$ of the data could not be defined and is therefore marked as other. 


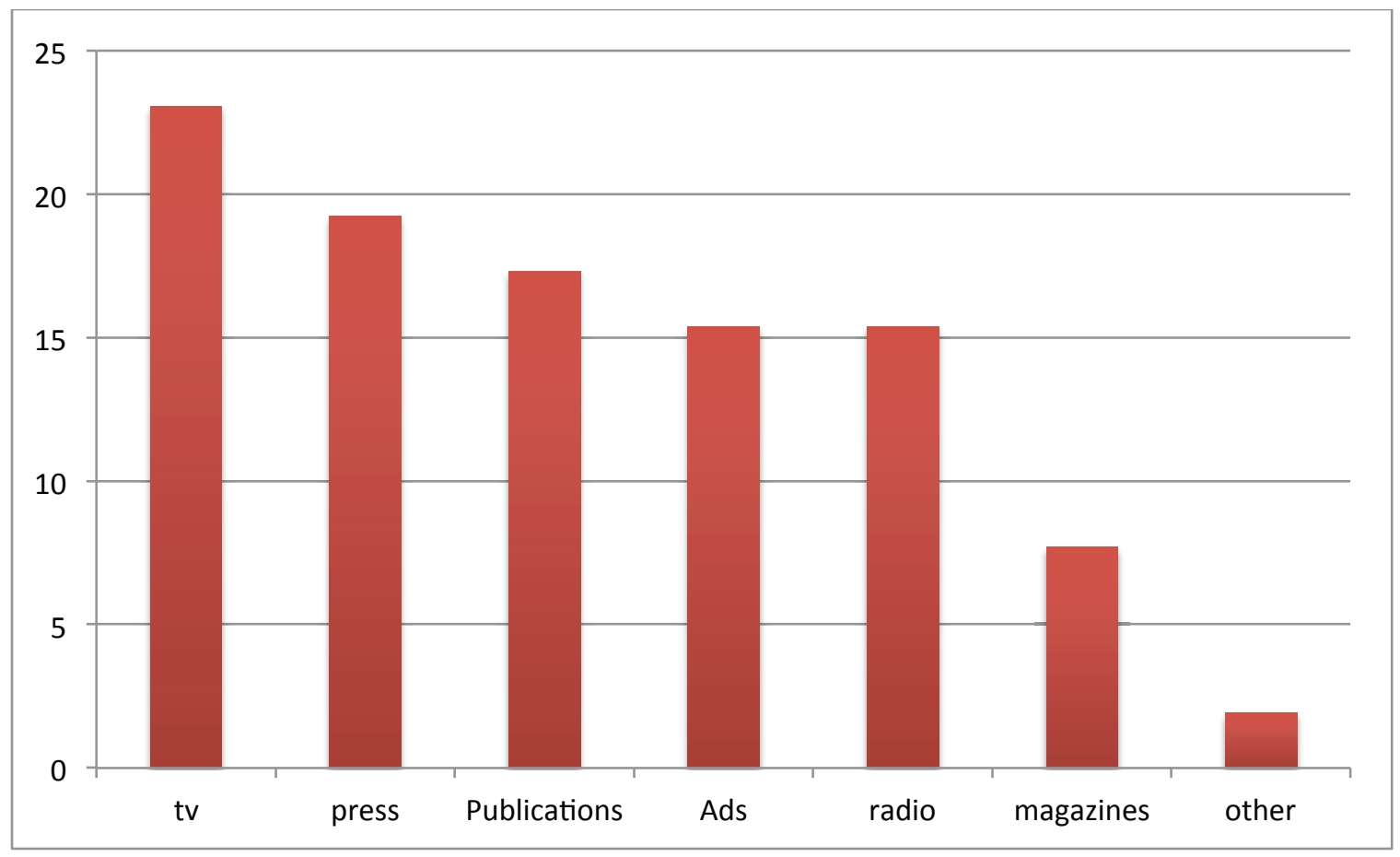

Figure 11 Breakdown of the Various Medial Channels Used by Living Labs

Of the 172 data points identified for the communication construct, 52 phrases were categorized as a form of media communication. Using the method in chapter 3 section 3.4.5, the data was grouped into two five channels, Television, News Press, Publications, Radio and Magazines which were apparent from reviewing the phrases. The key words used to place the phrases into the television channel were: TV, channel and television. The News press channel was identified by a search of key words such as: press and news. Publications were identified via: publication, journal, publish, and scholar. Phrases were categorized into the radio and magazine categories based on their title as key words. This process left 9 points of the 69 to be categorized. After several considerations, it was decided that 8 of the 9 points could be grouped together on the premise of their intended objective, promotion and where therefore termed Ads. The remaining point was left as other. 
Like the online channels, the media communication channels are self-explanatory. However, the three categories, press, publications, and magazines could be overlapping. However, they were separated based the following definitions. The press category refers to news channels either in the form of newspaper or digital form. In comparison, publications were specific to academic journal papers published in a peer-reviewed organization. Lastly, while magazine may be considered press, because of the specificity in the phrases, they were grouped independently.

\subsubsection{In-Person}

Figure 12, In-Person Communication Channels, plots the distribution of the various channels used to for communication via a face-to-face method. The nine channels have been identified as follows: events (24\%), conferences $(18 \%)$, seminars $(13 \%)$, workshops (11\%), sessions (9\%), lectures (4\%), expositions (4\%), courses (4\%), and tours (2\%). $9 \%$ of the data that was regarding In Person communication could not be categorized into one of the identified channels. 


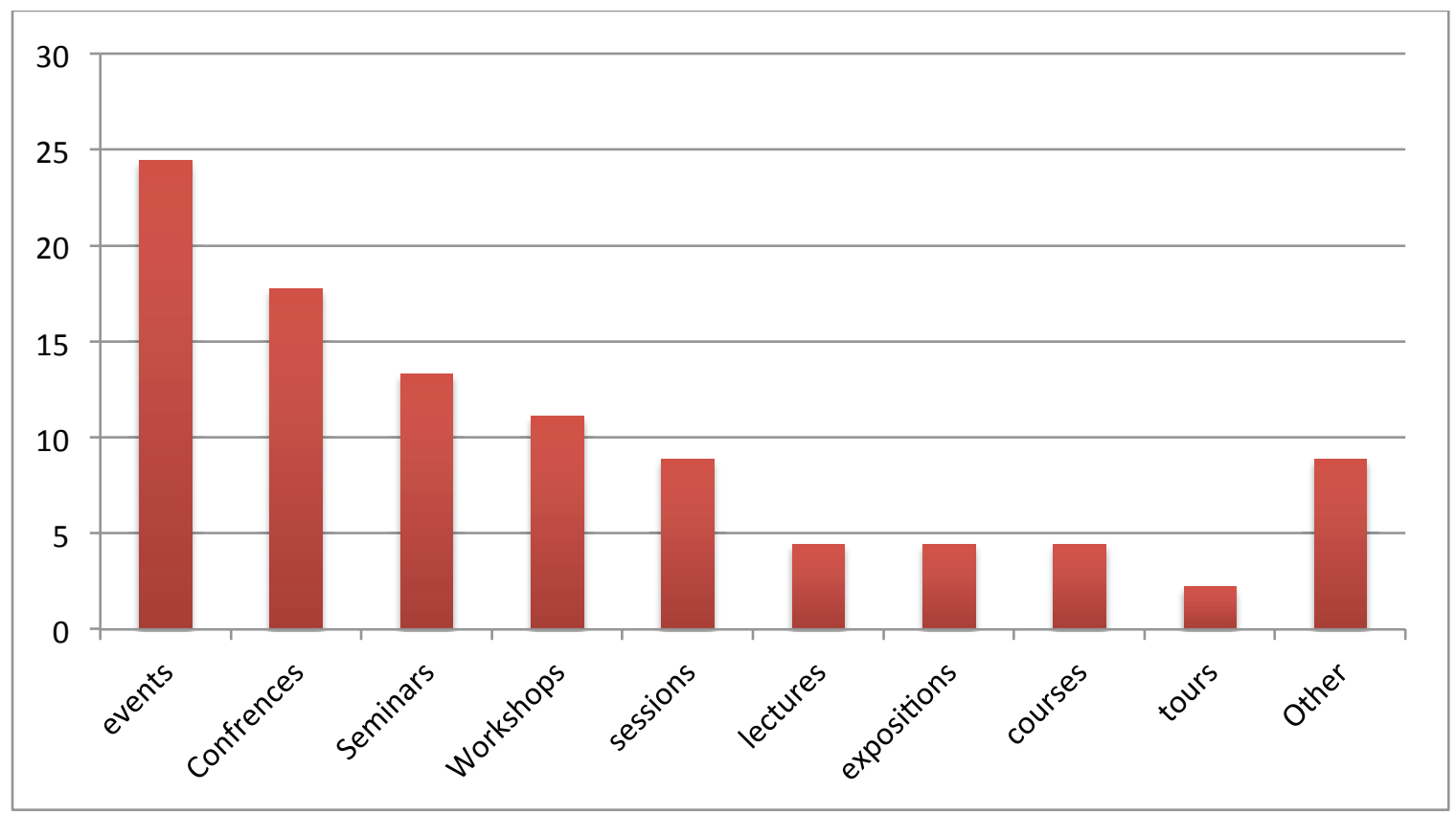

Figure 12 Breakdown of the In-person communication channels

Of the 172 data points identified for the communication construct, 45 phrases were determined to be referring to in-person communication. Compared to the other modes of communication, most of the data for this category were one-word sentences and not complete phrases. It was therefore easy to identify the various themes or channels by a subjective review of the data. I determined, based on common occurrences that the following channels existed for in-person communication: events, conferences, seminars, workshops, sessions, lectures, expositions, and courses. Using the method in chapter 3 section 3.4.5, and each channel's title as its key word, the data was counted and plotted against each other by percentage. The event category was the only group that required several key words as they encompassed various notions: events, talks, live and face. 
Each category describes exactly what its incorporating data explains. Data that was not specific, or did not explain in what way the face-to-face interaction commenced, was grouped under the events category; the generic and all-encompassing group of face-toface interaction. The data included for this group, talks, live demonstrations, consultations and more. The other category is miscellaneous data that does not appear to have relation to each other and includes phrases like "their work".

\subsubsection{Usage of Communication}

Figure 13, Reasons for Communication, measured what the data suggests is the purpose of the great investment of the communication modes. Comparing the points mentioned from the cases, it appears that the number one reason for the use of the communication modes is to connect/engage (37\%) with its members. The next most common reason (28\%) living labs invest in their communication modes is to update members of the activities of the lab. $17 \%$ of the data suggests that the living labs will use their communication investment in promotional campaigns which is distinguished from its use in developing its member's brands as indicated by $5 \%$ of the data. Comparatively, $7 \%$ of the data mentioned the use of the communication technology and modes as a means to manage the work between the members. Lastly, $4 \%$ of the data showed that the labs will use transmit educational activities and materials through their communication channels. Note, $3 \%$ of the data could not be categorized into a specific reason. 


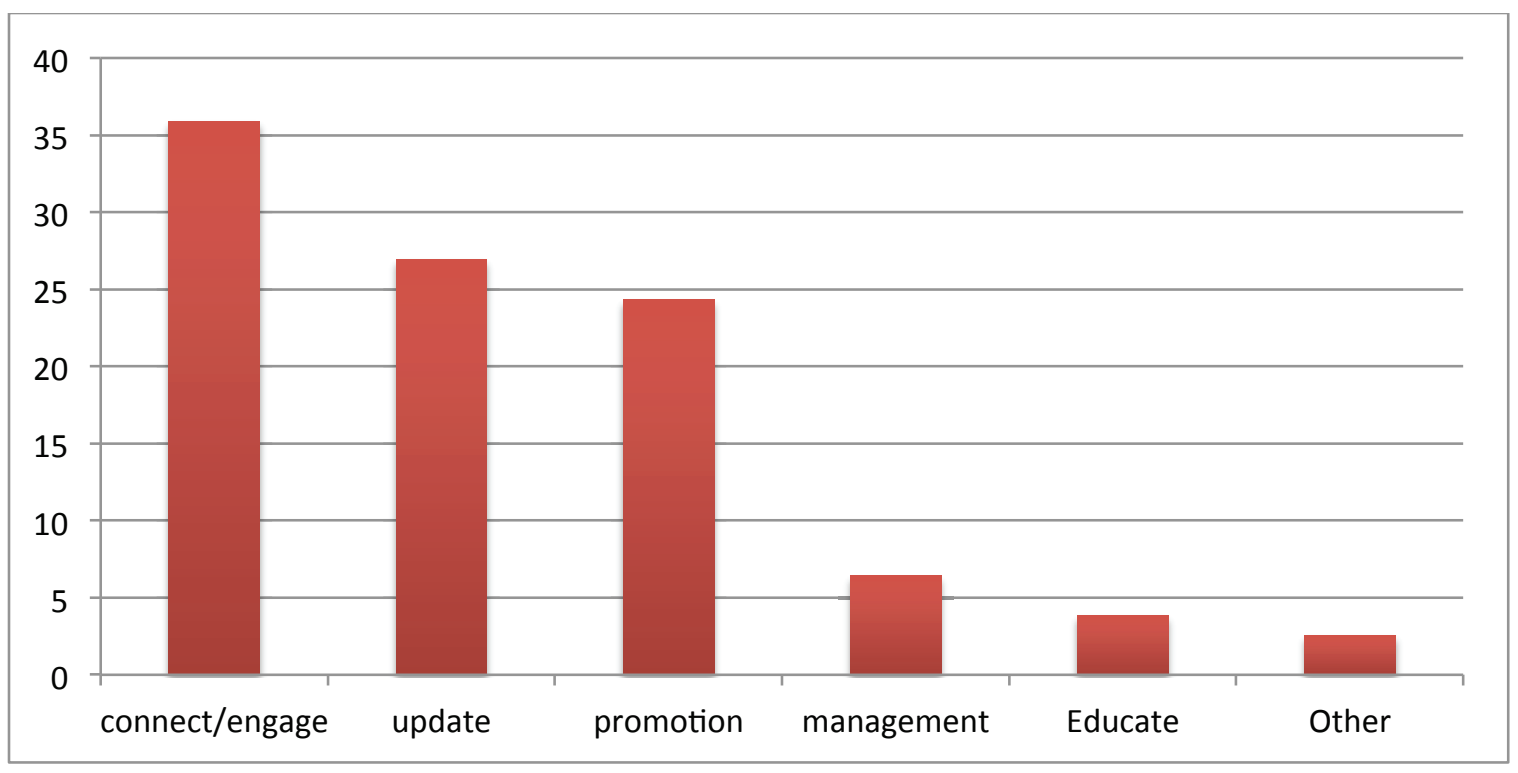

Figure 13 Observed and Measured Uses of Communication Resources

The data was not exclusive to determine the various modes of communication and the channels through which they operate. Instead, it was possible to quantify the data regarding the reason for communication using the method outlined in Chapter 3 section 3.4.5. In addition to the 172 data points extracted from the cases regarding the modes of communication, an additional 40 points were pulled in regards to "why" they use or "what" they do through their communication. The points were subdivided into a total of 75 points using an iterative process of categorizing the data. Using the other column, whenever a phrase was grouped into more than one category, the phrase was split into its respective reasons. The reasons categories were both subjectively determined by a careful examination of the phrases and a text analysis using key words. The three categories that were easily determined through subjective analysis of the data were: update, engagement and promotion. To determine if a phrase was to be categorized under update, it was screened for words that referred to awareness regarding on going activities. The key 
words used for this category included: update, activities, highlights, progress and dissemination. In like manner, the connect/engage category was screened for key words that referred to the interactive communication: connect, two-way, engage, perception and feedback. Keywords used to identify phrases in the promotion category were: promotion and event. Based on reoccurring words within phrases, and was apparent that three more categories could be created to group the remaining phrases. These categories are: Management, Branding, and Education. Phrases that had the key words, meeting or collaborate were grouped under collaboration. Phrases that had key words, control, manage or monitor were placed into the management category. The branding category was identified by the word recognition, and the education category used words such as: teach, educate, showcase and demonstrate.

It is apparent from the data that the primary use of their communication activities is for the purpose of engaging its members to achieve open dialogue for collaborative work. The data shows that the living lab tries to reach out to members and form a two-way communication channel. To the lab, it is important to have such an engaging and open form of communication to hold a meeting of the minds where they can brainstorm ideas, and gain valuable feedback and thoughts from its members.

The next most common use of the labs communication activities is for the purpose of updating their members and fellow citizens of the progress of projects or the ongoing activities of the lab. These updates are pushed out as news articles that highlight success stories and/or the effectiveness of a project, and the details regarding the R\&D progress 
of the undertaken tasks. Additionally, the labs will update its members regarding upcoming events or changes to the labs details including members information.

The third most common use of the labs communication effort is self-promotion. The lab uses its channels, online, media networks and in-person efforts to raise awareness about its activities, advertise the organization and reach new individuals to encourage new memberships. In like manner, the lab also invests in branding efforts to gain public recognition and legitimize their position (and that of its members) in the targeted industry.

Not surprisingly, the data did reveal that the communication channels used by the labs are also tools for managing lab activities. For example, lab websites can be used as a management system during idea generation, specifically for the purposes of collecting and appraising the ideas. Websites can also act as a cloud database that hosts the necessary content generated by the members, including videos, images, and other published works. Lastly, it is through their channels that they can track the ongoing projects and share free software to continue other management solutions.

Lastly, living labs use their communication channels to help educate its members, disseminating best practices and demonstrating new technologies (their usage) and techniques.

\subsubsection{Infrastructure Construct}


The data regarding living lab Infrastructure could not be efficiently analyzed due to the type of data collected. Although there is significant data regarding the used tools and equipment, it is not properly formatted for correct codifying making it significantly difficult to content analyze for patterns. What could be identified from subjective review of the cases is type or theme of infrastructure technology.

The data appears to have five types of infrastructure tools: facilities, networks, hardware, software and sensors. All living labs appear to have facilities, dedicated or shared, to host in-person activities such as events, workshops, and testing in a test bed. These facilities could be owned by a stakeholder who permits its use by the living lab, or it could be owned by the lab. Networks more commonly referred to as ICT, are the servers used to host the web technologies and data that effectively facilitates collaboration. Hardware, software and sensors are the technologies and/or tools that vary from lab to lab depending on their intended research. Sensors particularly are the technologies used within the test environment for observing user behavior and collecting usage data.

\subsubsection{Methods Construct}

Figure 14, Methods Categories, breaks down the relative themes found in the data regarding the methods the lab uses for innovation. The nine themes identified, in respective order of largest number of data points to lowest are: Data collection (20\%), Commercialization (15\%), Idea generation (15\%), Testing (10\%), Design (10\%), 
Communication (9\%), Support (7\%), Motivation (6\%), Attracting Participants (6\%), and Ethics (3\%).

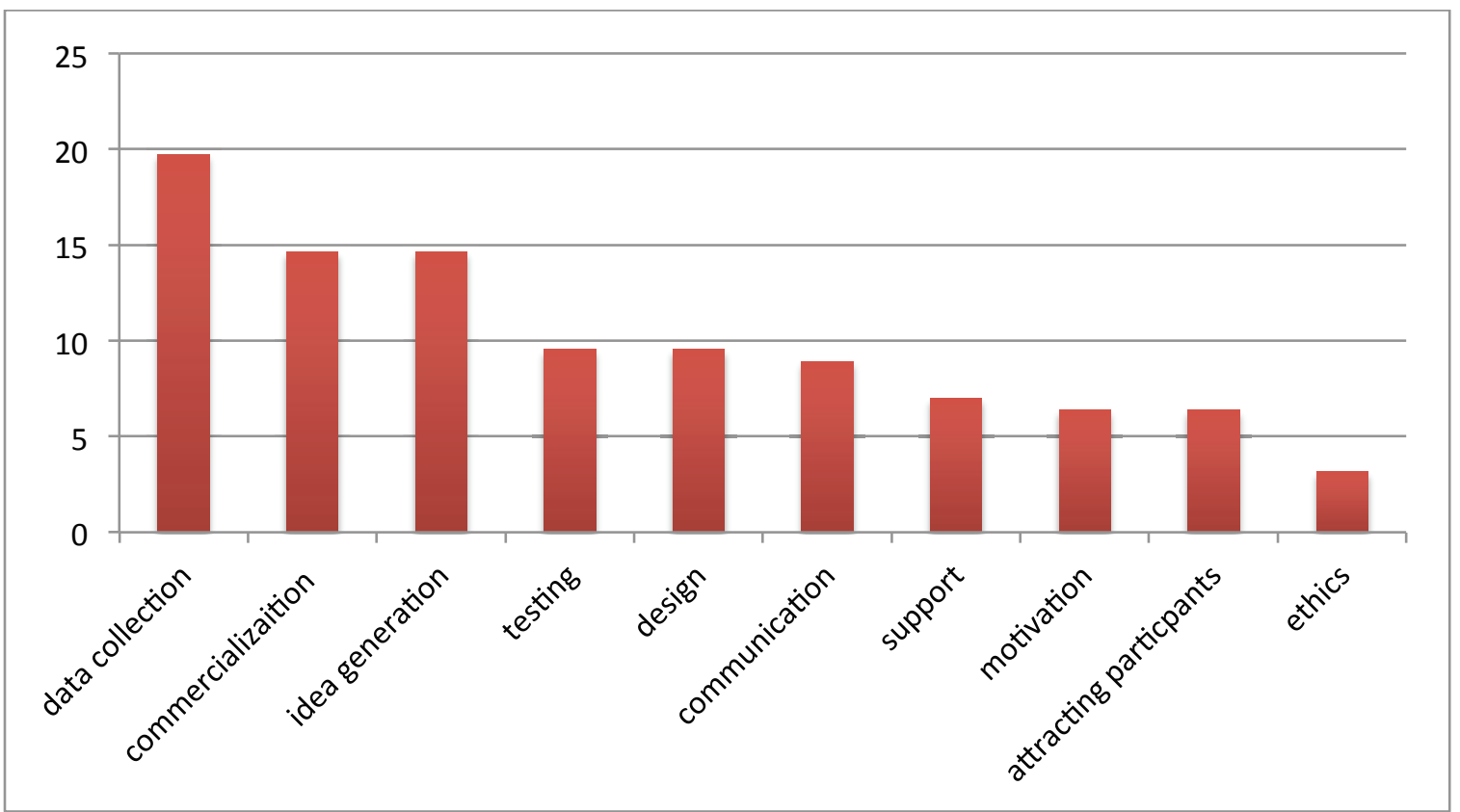

Figure 14 Observed and Measured topics relating to Living Lab's Innovation Methods

There was a total of 190 data points collected from the cases regarding the methods used to develop innovations within the living labs. The data was manually categorized into the groups by subjective decisions based on their context. 33 data points could not be categorized due to the scattered nature of their context and where therefore excluded from the results making all comparisons relative to 157 data points.

\subsubsection{Data Collection}


Figure 15, Data collection themes, further details the specifics of what the data collection category encompasses. The data was split between feedbacks, monitoring and databases.

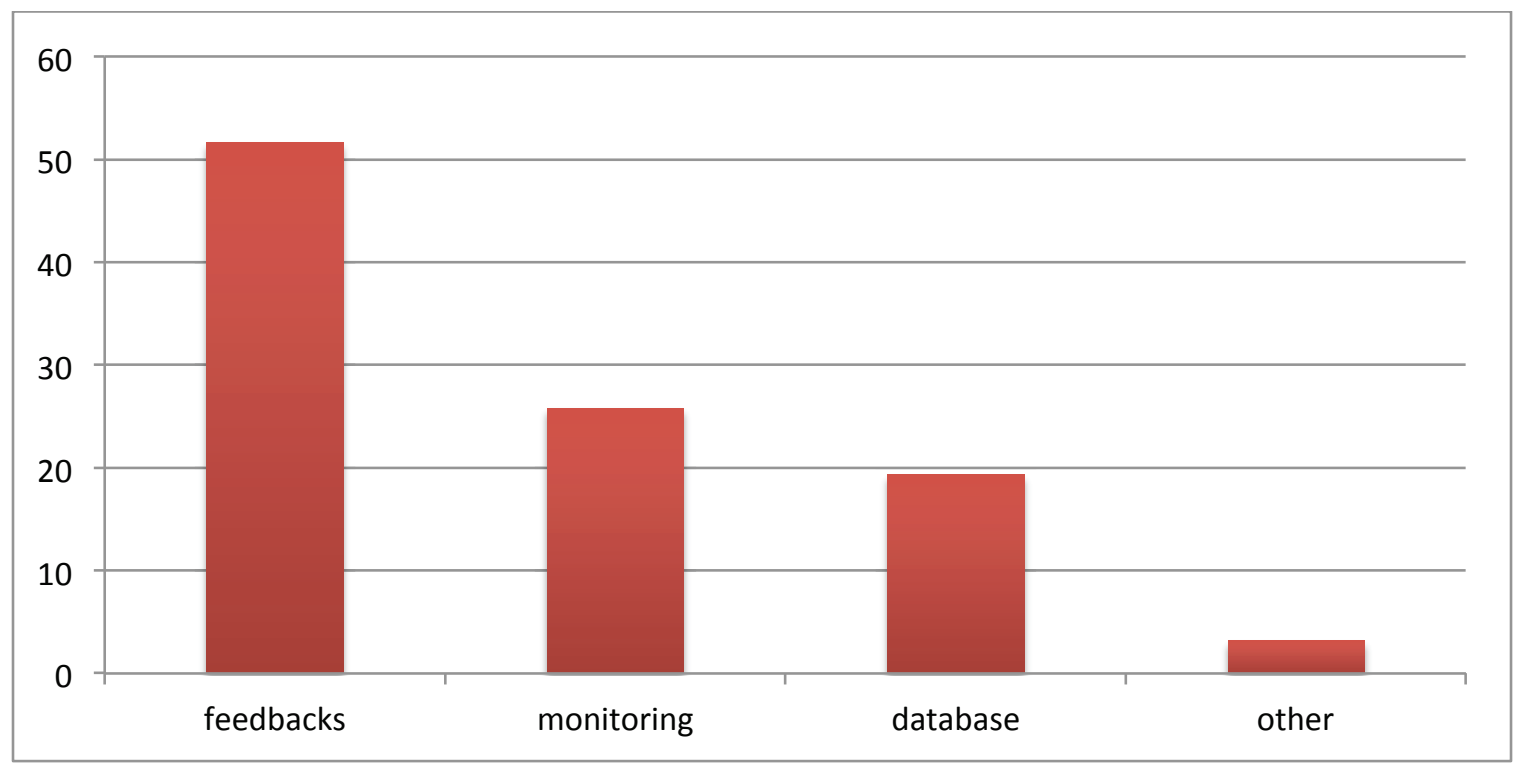

Figure 15 Observed and Measured categories of Data Collection Techniques

With $52 \%$ of the data points describing data collection, feedback was the primary discussion of this group. According the data, most of the information collected is the feedback of the users. The feedback is collected through several means. They use focus groups, meetings, surveys, questionnaires, one-on-one interviews, workshops and forums. Another collection technique is observational monitoring. If users are utilizing digital technology, the labs can embed monitoring bots that track and analyze patterns in the users usage of the product. In addition to the backend monitoring, the labs can use webcams and other sensor technology to observe the users interaction with the products and environment. In similar fashion, the lab uses the data that is collected via web 
technologies and the information shared by users through the forums to run data analysis to help extrapolate key details for innovations.

According to the data, as seen in Figure 16, Source of Users, living labs obtain their user participants through three sources; Associations (50\%), Random uncontrolled means (30\%) and through events (20\%). The number one source comes from the pools of individuals that member associations have access to. The individuals are committee members or actual clients, volunteers or actors of the association. The lab taps into the association because of the pre-established connection they have, and to comply with legal policy of ethics. The next most commonly form of gaining participants is at random when the lab gains access to regular citizens through the use of digital technology. Lastly, labs also gain user participation during the hosted events of the lab.

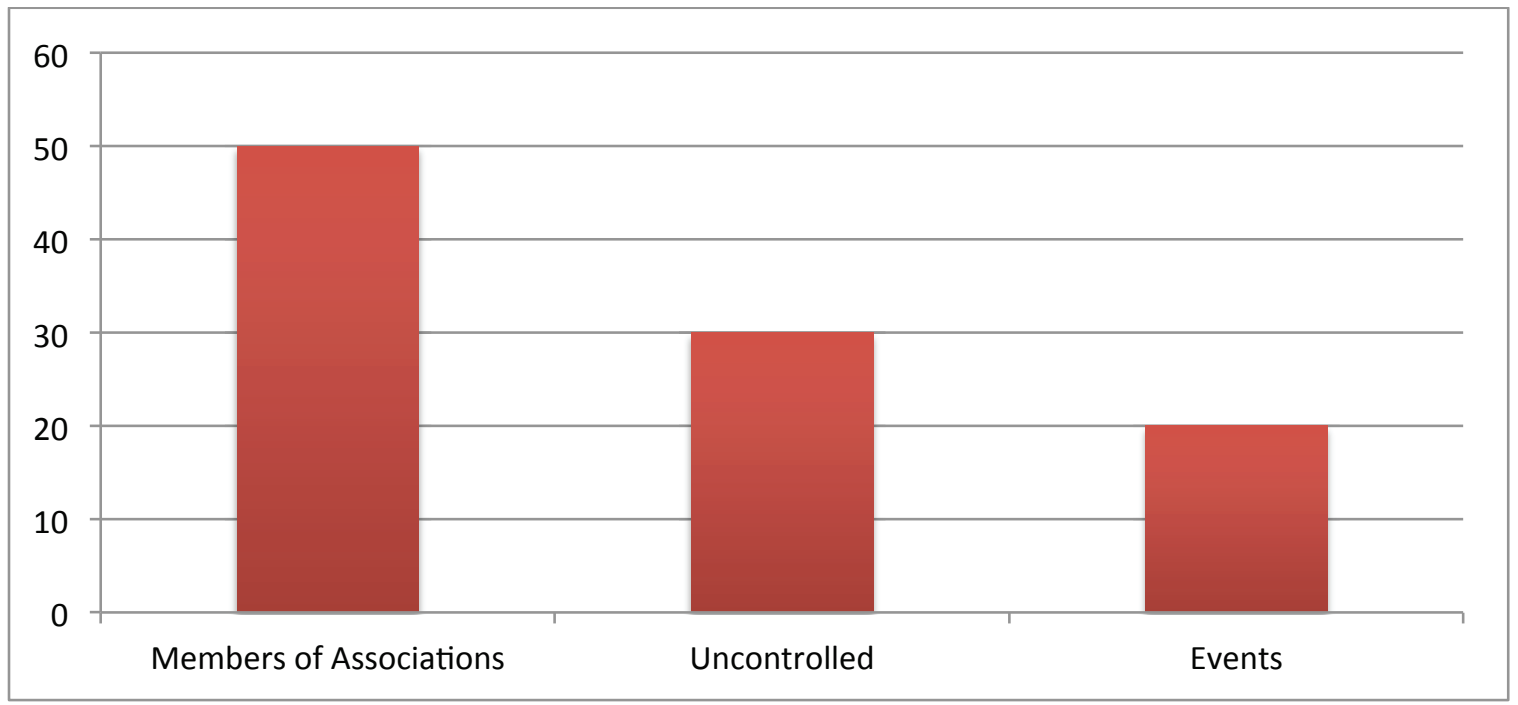

Figure 16 Observed and Measured Sources of Users 
Because the lab does conduct research both with and on users, it is vital that the lab follow ethical protocol. $3 \%$ of the data explain that Labs will inform the users of the ongoing project and will ask for voluntary consent in written form prior to their involvement. Living labs will allow the users to withdraw from the research at any time. They also work with companies and public service to ensure they are complying with all necessary procedures.

\subsubsection{Idea Generation}

Figure 17, Idea generation Objectives, distributes the data regarding idea generation into four categories: Set Requirements (45\%), Solution Discovery (21\%), Problem Discovery $(14 \%)$, and Other $(21 \%)$.

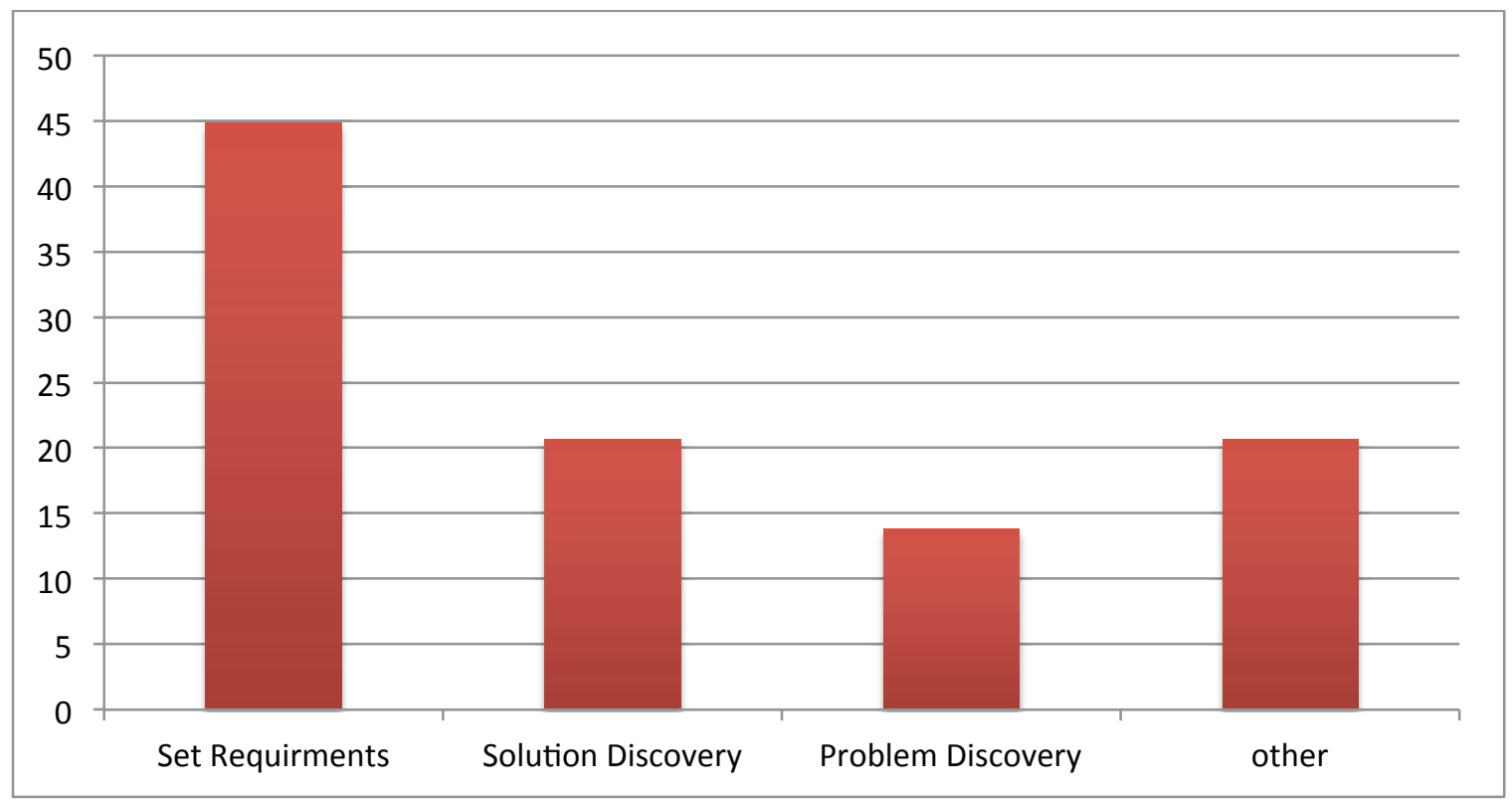

Figure 17 Observed and Measured Idea Generation Objectives 
The data reveals that the combined effort of academia, business, policy makers and users, the lab establishes a set of needs prior to undertaking a project. The members are engaged to express their problems and needs and to brainstorm possible solutions. Through focus groups, experts explain the technology trend and the expected future market demand. Researchers and professionals contribute their knowledge to further guide the brainstorming. Together with citizens, users and young individuals, the lab formulates new and innovative solutions ideas that could possibly be undertaken. The members are consulted prior to the undertaking of any project to set the basic requirements while designers set forth the technical limitations and possibilities. The lab uses courses, workshops and other forms of group activities to stimulate communal brainstorming and sharing of ideas.

\subsubsection{Design}

Figure 18, Designer Groups, reveals the three different entities that transform needs and requirements into innovation solutions. As seen from the Figure, small and medium-sized firms (SMEs) are the primary developers (68\%) of the innovations, universities second $(21 \%)$ and users the least $(11 \%)$. 


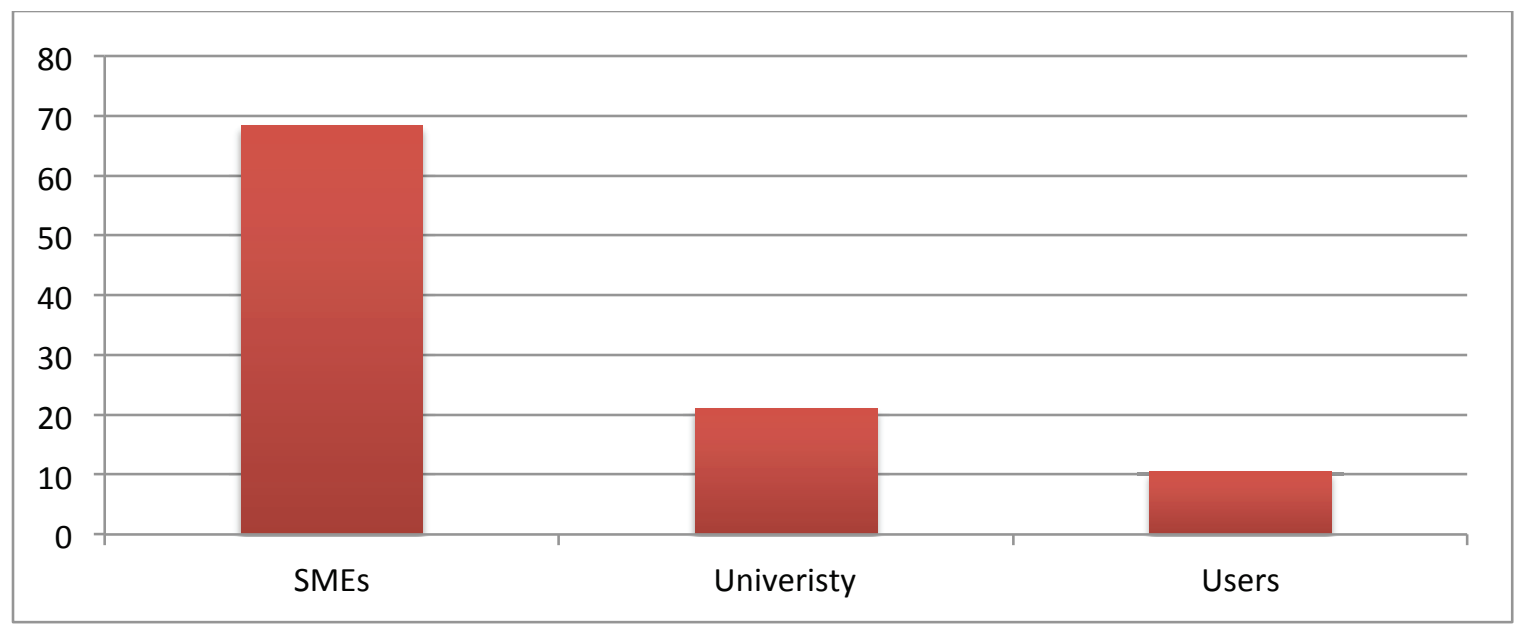

Figure 18 Observed and Measured Stakeholders who carryout Design Activities

SMEs often consist of expert business personnel such as engineers, and executives of $R \& D$ firms that make them optimal candidates for the role of designers. They are the ones who take the users established needs, requirements and feedbacks and incorporate them into a polished product. The development of the product often occurs in the facilities of the firms. Likewise, with their extensive resources, academia can also help design the solutions using their expertise and facilities. Academia harnesses the creativity of their professors and students through the courses and develops the innovative solutions for the living labs. Although the data is not detailed, it does show small evidence that some of the innovations are developed in the conjunction with the users.

The lab tests all output innovation with users in real life environments. Often, it is academia the guides the research, but incorporates members expertise is establishing benchmarks to test the innovations against. The lab monitors and collects the feedback from the users and sociologists analyze this data to better understand the impact of the innovation in a real life setting. Feedback often includes perceptions on the usability and 
functionality of the innovation as well as comments of the process of use. Sometimes labs will use independent organizations or individuals that have no stake in the process to guarantee unbiased results. The testing phase is an iterative process that cycle between receiving feedback and redesign for retesting.

According to the data from the case studies, it appears that the commercialization techniques are up-to the participating firms who have the expertise and resources to launch the innovation for revenue generation. It is the entrepreneurs' obligation to produce the business model and plan and take the innovation to market. Nonetheless, the living lab still aids in this phase buy establishing the test group (users) as the initial market segment and using their marketing team to promote the product and encourage members ("effective people") to help in the commercialization process. The lab will use the participating members who are "effective personalities" in the media to get the word out and broadcast the new innovations.

\subsubsection{Facilitation}

The lab uses web technology to facilitate the process from idea generation to full commercialization. The lab uses social media such as Facebook, and twitter, to organize and connect with the users throughout the product development cycle. Much of the information is trafficked through the web portals to express ideas, feedback and general knowledge during the innovation process. Nonetheless, the lab encourages in-person 
communication to improve the information exchange. Official papers and documents are shared between the lab and participants.

The lab provides supportive activities to the participants during the development cycle. They provide training, workshops and learning to empower the users and raise their level of skill and knowledge regarding the innovation. Business provides tools and technical support staff to aid the users during their experience; this maintains an open relation that gives deep insight into the users experiences. Lastly, professionals help by providing their evaluations and knowledge.

The lab motivates members to participate through different techniques. One method they use is a bottom-up mixed with top-down methodology to empower users and let them take lead. In similar way, the lab identifies lead users in communities and uses their influencing skills and position to persuade members to contribute and adopting new practices. This incurs the stewardship of such user communities on the labs part. The labs also reward systems to help motivate participation. They can offer monetary rewards or its equivalent; through the use of the service or product for free, when the user participates. They also use gamification techniques as well as idea jams to stimulate a sense of excitement for contribution and participation. 


\section{Chapter: Discussion}

Chapter 5 is an explanatory section that clarifies the lessons gained from the research and contributions made to theory. In the sub-sections that follow, Section 5.1 discusses the results from applying the case study approach to the analysis of real-life living labs for the identification of patterns. Section 5.2 interprets the findings of each construct for the continued understanding of living labs and compares, to literature, the added knowledge to the field.

\subsection{Results from Case Study Approach and Content Analysis}

The results described in Chapter 4 are the output of Case study Research and Content Analysis. Case study approach is best used to examine phenomena's that are poorly understood; such in this case of living labs concept. Starting with lessons from theory, initial constructs, that describe innovation concepts, were synthesized to guide the examination of the living lab cases. During within-case analysis, the constructs were modified to better describe the recurring information found in the available data. After compiling case-write-ups and subjectively determining possible patterns, content analysis was used to measure pattern occurrences.

Using the combination of case study and content analysis approach, this research was able to find several patterns among living lab cases and thus contribute a set of nine constructs that characterize living labs concept. The nine constructs are: Living Lab 
Objectives, Governance Responsibilities, Collaboration Philosophy, Stakeholders and Actors, Financial Structure, Advantages of Living labs, Communication Resources, Operational Infrastructure and Living lab Methods. The sub patterns within the constructs further contribute new understandings of living labs describing important elements that single out living labs and their prevalent operations.

\subsection{Construct Contributions}

To best understand the significance of the findings and better understand contribution to living lab theory, this research enfolds current literature knowledge as discussed in the

case study approach. By drawing on connections between this research and theory, we can confirm or disprove pre-existing notions of living labs and gain a better understanding of the concept. The following section will discuss the respective findings with their relative literature equivalent and contributions made based on the constructs.

\subsubsection{Living Lab Objectives}

One of the contributions of this research is the Objectives Construct of Living Labs. Objectives of the living lab are the intended goals or the advantageous benefits sought from the output of the innovation concept. According to literature, there could be considered several goals living lab aims to offer and that participating members hope to gain. In respective order of perceived importance, these goals include: test environment, innovation methodology, new knowledge (particularly product usability), business 
support, and a collaboration framework. Patterns measured under this construct found that that the goals in respective order are: Collaboration, Social Impact, Business Development, Economic Development, User Impact, ICT, Test Bed and Framework.

First and foremost, it is believed that the main objective of a living lab is the establishment of an arena (or test environment). This is due to the unique nature of the innovation concept being grounded in real world experimentation. In addition to the arena, the living labs are meant to provide its members with a procedural innovation methodology to optimize experimentation within the arena. However, based on the observed results, this notion appears to be incorrect as both, the arena and innovation methodology (framework), were measured to be the least intended goal. Instead, it was found that the main establishments (of all the goals) of living labs are three fold: Collaboration, Social Impact and Business Development.

As hinted towards in the literature, one of the living lab's objectives is to bring together a diverse network of stakeholders, and facilitate innovative collaboration between them under a fair and effective framework. Many of the cases analyzed suggest that this is the first and most important goal as it simplifies the sharing of ideas, knowledge and experiences as well as resources to help with the innovation process. Living labs sets this as the main goal because it also the means by which it can pool the necessary incubation spaces, state-of-the-the art technology and knowledge databases. 
An interesting find, which does not appear to be discussed in literature, is that one of the main objectives of living labs is Social Impact. How living labs positively influence their community's environment and way of living is what Social Impact means. Just as important as collaboration, it turns out most, if not all, living labs intend on improving the regions status and quality through urbanization, development of environmentally clean alternatives and growing the communities involvement and more.

Business development was found to be a main priority of living labs. Their goal is to offer the necessary resources and services that can support the growth and development of participating firms. Literature considers all operations of the living lab to be in, one form or another, a means to support firms and their development. Therefore it does not particularly specify the degree of importance and quickly mentions that it includes employee support, supplier value, and managerial tasks. However, based on the findings, we can now offer a more detailed explanation of the directed business development goals. The findings specify that living labs help support firms through incubation space, market trend analysis, equipment and education. The help they offer in discovering user needs, testing and validating their designs and commercialization support are also included in this category.

The data was not able to support that knowledge generation was an objective of living labs. Knowledge generated for business development could be considered, but literature focuses on knowledge generation being scientific in nature and geared towards bringing benefits to consumers. It is therefore reasonable to suggest that the measured objective 
User Impact be in part, supportive of the literature's mention of knowledge generation because of the shared relation of producing improvements for users. In this manner, the research does support the literature in claiming that living labs aim to generate knowledge, but it becomes important to differentiate the two and mention that it was not a significant goal.

Lastly, the findings revealed that living labs also have the goal of establishing and growing the region's economic condition. This is a revelation, as the literature did not mention that living labs could be formed for the purpose of attracting investment and business to the region.

\subsubsection{Governance Structure and Responsibility}

Another contribution made by this research is the identification of the Governance Construct. Previous research mentions the importance of a proper governance structure for living labs in order to avoid dependencies and enable win-win strategies. The governance of the living lab is important because it describes the manners in which decisions are made and how activities of the operations are managed. Based on literature, little is known about the governance structure of living labs, other than that they take on a form of up-down structures and that the lab itself is a legal entity forming a public-private partnership. 
Through content analysis, this research was able to contribute findings regarding the legal structure of living labs. There exist four possible legal structures: private, public-private partnership, public-private-people partnership and public ownership. Compared to literature, the findings support the popularity of a privately incorporated and owned living lab structure.

Moreover, this research contributes an explanation of the responsibilities and duties of governance groups in living labs. In short, governance groups are responsible for setting the vision of the lab and ensuring all activities fulfill the mission statement. They take on administrative roles in organizing and managing resources as well as establishing constitutional rules by which the lab operates. For detailed description please see Section 4.3.2.

This research lastly supports the difficulty of standardizing the governance structure. Through cases analysis it was possible to recognize living labs mostly have top-down decision-making structure, but because the decision teams were composed of participating members, it could be considered up down.

\subsubsection{Collaboration Philosophy}

This research contributes the Collaboration Philosophy construct to describe the Intellectual Property Rights and openness of living labs. Literature has mentioned two important topics regarding the collaboration philosophy of living labs: Intellectual 
Property Rights and Trust. The philosophy construct sought to explain the mentality of the organization and how it addresses openness and collaboration.

According to research, problems arise in collaboration initiatives such as this because of the shared resources, knowledge, and efforts, which make it difficult to appropriately compensate each participant. Additionally, policy makers have not yet outlined set laws to dictate the dynamics of such collaborations.

This research therefore contributes the identified patterns that help clarify how the living lab mitigates IPR management issues. The findings show that there are several ways to manage IP. These management techniques can be found in Section 4.3.3.1. The most common technique found to address the issues was Consortium Agreements. These agreements set forth rules and regulation as to what portion of IP is owned by each member, the terms to use others and developed IP and how sensitive information is handled. Because the rules are formulated and agreed to prior to any innovation project commencement, it set an unbiased standard to which all participating members are comfortable with.

While literature emphasizes the presence of trust, it lacks to mention how to establish trust. This research therefore gives insight into how living labs nurture trust and collaboration within their network. Based on our findings, much of the data regarding innovation culture describes their investment into collaboration efforts. Living labs promote member respect and encourage active participation in all its activities to form a 
sense of community. They try to reduce as many barriers as possible to stimulate cooperation. The lab adopts an open mentality in their dealings, providing access to their knowledge database and use open-standards so all have access to free tools and are free to manipulate sources as they please.

\subsubsection{Stakeholders and Actors}

Part of this research was the identification of Stakeholders being an important construct to describing living labs. Although it was not possible to analyze the available data, it is apparent (based on the extensive and detailed amount) that it is an important topic and requires further research.

Living labs have a diverse group of actors. The literature suggests that the more diverse the group the better because of higher cross-pollination of knowledge for the generation of creative ideas. Diversity helps complement the stakeholders allowing for a better realistic social context. Previous research and this research agree that the involved stakeholders are often academia, government, companies, and users.

From the methodology construct of this research, we were able to identify that contrary to popular belief, users are not as involved as thought to be. Based on the case analysis, users only get involved in the ideation and testing/validation phases of the development process. In rare instances, labs involve users in the creation process, but this often refers to content production and not actual design. Instead, it was found, in agreement with 
literature, that design of innovations is the responsibility of the firms and technically capable persons (such as engineers).

\subsubsection{Financial Structure}

Originally, the financial structure was meant to discover how the innovation concept captures monetary value from its operations; it could have been sales of the innovation output, providing upgrades, maintenance, and/or support services. The focus of this research shifted to how the living lab financially supports its innovation activities and thus sought to answer "where does funding come from?" During the case analysis, it was apparent that it discussed funding sources versus business appropriation. This gave the opportunity to confirm research, which suggested that living labs need substantial funding and often attain it from public sources, and private investors.

Based on this study, it was found that in addition to public and private funding, living labs have structured a multi-revenue stream business model to become independent of the other sources of funding. Moreover, it we also identified five means to which the lab makes money: consulting, royalties, rent, membership fees and education. These findings contribute to literature suggesting the use of private business models for living labs and detail the makeup of the business model. The findings also show that private business models of living labs not only help generate income, but also are used as a metric to measure the health and success of living labs. This research briefly shows that money, regardless of the source, is not used for profit making purposes, but to offset the cost of 
the labs operations and equipment purchases. Business appropriation appears to still be in the hands of individual firms.

\subsubsection{Benefits of Living Labs}

The Advantages construct describes the benefits that participating members gain from their involvement in the lab. This research contributes the ten identified benefits and measured their relative prominence. As seen in the results section, these ten advantages are: business development, knowledge, resources, networking, validation, marketing, improved products, frameworks, social value and investments. According to literature, there are only six benefits to gain from living labs: innovative ideas, networking opportunities, market development and validation, financial support, improved processes and improved products.

As it can be seen, the literature confirms our findings showing that that networking, marketing and validation, improved products and financial support are benefits that living labs provide to their stakeholders. Business development, the highest-ranking benefit from our research, corresponds with improved process mentioned in literature. As part of business development, advisory teams and expert personnel, are provided to help support entrepreneurs with their business, this of course reduces mistakes and accelerates their business process. The four other benefits identified in this research are: knowledge, resources, frameworks and social value. 


\subsubsection{Communication Resources}

This research contributes a living lab construct referred to as Communication Resources. The communication construct was identified to describe the channels, technology and techniques used to network and exchange information. As previous researchers have explained, to enable innovation collaboration, the use of web technology is critical because it allows for synchronous and asynchronous exchange of information. They highlight the importance of such tools as wikis and blogs and other online facilitation tools that act as shared workspace. With such great emphasis on online technology, researchers have not examined other modes of communications that living labs could use. This research has identified two additional modes of communication and measured their relative use compared to online tools. Moreover, each mode was further analyzed to determine their most prevalent channel of use.

This research therefor complements, in addition to online means, the living labs investment in traditional media channels as well as In-person events. Following these modes, we now know that the main channels of communication of living labs are personal websites, Television networks and specially hosted events. There are of course many more channels in use; however, these are the most prevalent. Moreover, this research contributes to how living labs harness these channels for collaboration, which the literature appears to be silent on. For detailed findings, please see Section 4.3.7.

\subsubsection{Operational Infrastructure}


From the data collected, it was possible to identify this Construct as being important to the characteristics of living labs. However, this research could not sufficiently contribute to theory about the necessary resources and specialized equipment required to carry out the innovation activities. Based on case analysis review, we could subjectively identify five possible categories that infrastructure could fall within: facilities, networks, hardware, software and sensors. They could not be confirmed with content analysis due to complicated semantic coding.

\subsubsection{Living Lab Methods}

Living lab methodologies make the most researched and theoretically discussed topic concerning the phenomenon. It covers the procedural steps used for the inception, development and deployment of innovation. Cross analyzing multiple cases, this research was able to confirm this as a construct and identify ten themes from the data regarding the methodologies used among living labs. The themes help paint a picture to the general pattern of how a living labs conduct their experiment and or what is involved during the procedure.

As seen in the results section, the methods don't just involve the phases of ideation, design, development, testing and validating, instead, it includes acquisition of users, data collection techniques, supportive services, motivational activities and ethics. 
This research shows that users cannot always be selected using scientific means to satisfy maximum sample variation. According to our findings, participants come mostly from civic associations, uncontrolled sources (e.g., housing authority), or voluntary participation from events. The ideation phase, gathers together all members, users, designers and experts to help explore pain points and possible solutions, and set specifications and requirements for the solutions. Development is done by professionals (SMEs and engineers) and is handed to the users to be tested in real life settings. Because researchers from academia that are most experienced with the scientific method, they lead the experimentation process for optimal results. Social analysts for interpretation analyze the collected data. The development and testing phases is repeated until a satisfactory product is produced. The product is then handed over to the company to commercialize and exploit the product.

The data collection for the full process is thought to be done through ICT technology via a monitoring process. However, this research shows that most of the data is collected qualitatively through traditional means: focus groups, surveys, questionnaires, workshops, and interviews. Traditional data collection gives insight into perspectives and feelings. These collection methods can be transferred over to online means, over Facebook and Twitter. Online monitoring is used during the usage of the innovation. Such monitoring could be done through camera observation or through backend data collection if the technology is digital. 
The research adds that living labs provide supportive activities to members during the development cycle. We find, compared to literature, support is not just for the education and training of the users, but also as a means to stay in touch and gain interactive knowledge. 


\section{Chapter: Conclusion, Limitations and Future Research}

Chapter 6 provides the final thoughts about the research described in this thesis, mentioning the limitations of the research and suggestions for future research. Section 6.1 is the conclusion; Section 6.2 lists the limitations and Section 6.3 focuses on the suggested areas for improvement and further research.

\subsection{Conclusions}

Living labs is a relatively new phenomenon that has been around only for a decade. In that time, several researchers have attempted to study living labs and model its operations. To do so, each researcher has proposed a definition of living labs that best suited the goal of their research and therefore has resulted in a slue of varying meaning of living labs. Some researchers have defined living labs as 'user-driven innovation infrastructures' (Kareborn et al., 2010) while others have explained it as 'user-centric research methodology' (Erikkson et al., 2005). As further research is conducted and new knowledge is formed in the topic of living labs, these definitions can become very specified or vague. Corelabs (2008) defines and describes the concept as an approach with "functional regions where stakeholders have formed a Public-Private-Partnership ... all collaborating for creation, prototyping, validating and testing new services, products and systems". Comparatively, VINNOVA (2009) simplifies living labs as societal resources that set frameworks, experiences, and conditions to develop ideas into innovations. 
It was evident that researchers could not agree on a definition of living labs (cf. Nystrom et al., 2014), or identify constructs that characterize the structure or operations of living labs (cf. Dell'Era \& Landoni, 2014) and focus their research efforts. It was therefore the objective of this thesis study to identify the constructs of living labs that will not only help establish a more robust definition, but also aid in guiding future research.

Following a case study approach outlined by Eisenhardt (1989), this research examined forty living lab cases from ENoLL's publically available database. Using pre-developed constructs from literature, the cases were analyzed for commonalities and relevant information regarding their innovation operations. During the process, revised versions of the constructs that best described the living labs were developed based on the findings. Using content analysis and computer aid of Excel, the information regarding each construct was analyzed for frequency of themes and used to further develop the constructs and better define living labs.

This research did not use a specific definition of living labs to help guide the focus of the study. Instead, because it started from foundational principles (i.e. compared to other innovation concepts in multiple areas) and used a multi-case study to discover information about living labs, the findings of this research gave us better insight into living labs and uncover commonalities and disagreements between existing living labs and current literature. 
The constructs and their internal themes identified by this study give researchers and businesses different perspective to understanding living labs. Each construct reveals how living labs differs from other innovation initiatives and explains how it is capable of developing innovations. Using these constructs we can now attempt to better explain living lab's innovation concept. In summary:

Objective: Living Lab is an innovation platform that aims to deliver a collaborative framework for its stakeholder ecosystem and offers business development to entrepreneurs and firms while creating innovation for the betterment of society.

Governance: Living Lab can either be privately incorporated entity, a public organization or a public-private-(people) partnership, that delivers innovations through an up-down decision making structure.

Philosophy: Living lab is a collaborative innovation platform that utilizes open standards, sharing of knowledge and Consortium agreement to fairly manage collaborative innovation.

Stakeholders: Living Lab is an innovation ecosystem that brings together academia, government, companies, associations and users to pool together resources and collaboratively create innovations where, users bring innovative ideas, companies develop the solutions, and academia guides the real-life experimentations.

Funding: Living Lab is an innovation organization that gather their financial resources from public sources such as national research grants, private investors such as member 
companies and their own personal business model that primarily generates revenue through consulting services.

Advantages: Living Lab is an innovation platform that provides extensive business development, new knowledge, and networking opportunities. The platform provides investment opportunities to reduce development costs, helps to validate products and establish initial market demand through their stakeholder community.

Communication: Living Lab is an innovation platform that uses online, media and inperson modes (particularly, websites, TV and events) to exchange information between members (such as ideas and feedback) and lab updates to enable collaborative work.

Infrastructure: Living Lab is platform that uses extensive infrastructure including facilities, networking tools, hardware technology, advanced software, and monitoring sensors to conduct innovation operations.

Methods: Living Lab is an innovation platform that provides an innovation process including attracting participants, motivating, idea generation, design, testing and validation and finally commercialization. The process addresses data collection techniques and engagement methods.

Combining the definitions of the individual constructs, it is possible to form a more comprehensive and general definition for living labs: "A living lab is an innovation platform with shared resources and collaboration framework, that often organizes its stakeholder ecosystem into a legal structure that uses an up-down governance, open- 
standards, various communication modes and an innovation methodology to deliver business development, new knowledge and validated solutions."

\subsubsection{Implications}

The developed definition is formulated from a generalization of multiple cases versus singular living labs and is based on the most popularly discovered themes. The definition does not perfectly grasp the complexity and diversity of living labs because it is generalized from a sample of forty living lab cases. Therefore the definition should be considered a definition of best fit, leaving room for variances. Nonetheless, this definition serves the purpose of creating a baseline to best describe living labs and to be used as a starting point or comparison for future work within living labs.

The constructs and their respective themes identified by this thesis gives researchers more than just an understanding of living labs, but also a guide for future work. The constructs set focus points and starting knowledge (i.e. themes) for researchers to formulate specific problems pertaining to living lab to be further examined and developed into a more detailed theory or comprehensive practice. As each construct is researched further, they can become more refined and provide more insight into living labs innovation. Moreover, the more these constructs are improved the better the definition of living labs becomes and the easier it will become to generate frameworks and models to encompasses the principles of the innovation concept and guide the formation and operations of a living lab. 
While the definition gives technology entrepreneurs a better understanding to living labs, the constructs along with their detailed themes provide insights and better understanding to the innovation concept as a whole. Through this research the entrepreneurs now know the purpose of a living lab, the stakeholders and their roles in the platform, the structure and how decisions are made/influenced and the methodology used to develop innovations. Moreover, entrepreneurs can identify the particular advantages of living labs (business development support, access to resources and partnership networks, as well as product ideas, validation and commercialization) and focus their efforts to joining the lab that provides more of a particular benefit. In other words, technology entrepreneurs can now navigate through the living lab landscape enabling them to identify a lab that is aligned with their business trajectory and make an informed decision when joining a lab.

Most importantly, stakeholders are enabled to establish their own living lab by addressing each construct and choosing a particular theme for each. With a detailed explanation of the objectives, structure and operations of living labs, stakeholders can look into the implications of each theme and better form an innovation platform that best suits their goals, maximize their funding, and is aligned with their society and stakeholders to optimize the innovation process. Below are questions regarding each construct that could help the process of developing a living lab: 
Table 8 Questions to guide establishing a Living Lab

\begin{tabular}{ll}
\hline Construct & Question \\
\hline Objective & What does your living lab aim to achieve? \\
& Will it have multiple objectives? \\
& Is your focus collaborative framework, \\
& business development, Social Impact, \\
& Economic Development, User Impact? \\
\hline Governance & Will you use Top-down or bottom-up \\
& governance? \\
& Will representative members of the lab \\
& comprise the hierarchy? \\
& What kind of legal structure will the lab \\
& take? \\
\hline Funding & Will IPR be managed through Consortium \\
\hline Philosophy & agreement, Case-by-case, open source etc.? \\
& How will trust and collaboration be \\
& investment? Or Revenue model? \\
& Access to databases? \\
\hline What will be your primary source of \\
\hline
\end{tabular}




\begin{tabular}{|c|c|}
\hline & Facilities or Technology to be leased? \\
\hline Advantages & $\begin{array}{l}\text { What benefits do you propose to your } \\
\text { stakeholders for their participation? }\end{array}$ \\
\hline Communication & $\begin{array}{l}\text { Will you be using Traditional modes of } \\
\text { communication (i.e. TV etc.) to deliver } \\
\text { information or focus on Web technologies? }\end{array}$ \\
\hline Stakeholders & $\begin{array}{l}\text { What actors do you need? } \\
\text { What are the roles of each actor? } \\
\text { Will the user be more involved in actual } \\
\text { design process rather than just ideas } \\
\text { generation and testing? }\end{array}$ \\
\hline Infrastructure & $\begin{array}{l}\text { Does the lab have physical facilities' to } \\
\text { host face-to-face events? Do you have } \\
\text { sensors to monitor the users in the test } \\
\text { environment? }\end{array}$ \\
\hline Methods & $\begin{array}{l}\text { Where are you getting participants? } \\
\text { What ethical procedure is being used to } \\
\text { conduct experiments on users? } \\
\text { Who's creating the experimentation } \\
\text { procedure? Academia? } \\
\text { How is the lab going to produce and } \\
\text { consume the product? }\end{array}$ \\
\hline
\end{tabular}




\subsection{Limitations}

The following limitations of the research have been identified:

1. Due to time restriction for completion of thesis, criteria were set to limit the number of cases analyzed. As with all empirical studies, if a larger data set was used, resulting patterns discovered may become more refined and generalized and therefore better descriptors of living labs. Moreover, additional cases provide more qualitative data that could be used for interpretation of patterns and constructs leading to a more detailed explanation for the obtained results.

2. Due to diversity in the living lab cases, unique information such as the technology used, are highly specific, making it difficult in codifying an accurate semantic theme frequency counter. Likewise, data that are nouns, such as names of stakeholders require additional research to determine their equivalent pronoun (user, designer etc....) before codifying an accurate semantic category counter. This problem occurred for the infrastructure and stakeholder constructs. The data requires extensive pre-editing and formatted correctly based on more generalized pronouns prior to analysis. This is requires heavy investment of time and effort. 
3. The human subjectivity of case analysis is a limiting factor. Interpretation of the qualitative data is dependent on the researchers current understanding of the subject and pre-existing notations brought into the topic. For this reason, content analysis was used to limit the biasing of human interpretation.

\subsection{Future Research}

Using the contributions of this research, we propose the following future work to be done:

1. Using the methodology outlined in this research and the key words used, the Constructs and sub patterns discovered here could be confirmed using a larger set of data.

2. Researcher may tailor their research and focus on the individual constructs or subpatterns to further the descriptive knowledge of the topic in relation to living labs.

3. The constructs may be applied to other innovation concepts as a guide to developing unique patterns for those concepts. 


\section{Appendices}

\section{Appendix A}

\section{A.1 A Cross-Border Living Lab on Territorial Marketing}

The Cross-Border Living lab on Territorial Marketing (CBLLTM) is a limited liability company located and operating out of the city of Reggio Emilia, Italy. Its sole intention, as mandated by local development policy, is to attract business investments into the regions and to build up from existing or scratch the economic sector of the territory. CBLLTM aims to provide services in the marketing domain both locally and internationally to effectively aggregate society, economy and environment together to achieve their mandated goals. CBLLTM vision is to provide services that will strengthen the local economy and tourist territories. CBLLTM currently provides consultancy to public sector organizations on ways to optimize the regions resources and develop external promotion campaigns. The Living Lab helps intermediation between different industrial districts of Europe to help foster collaboration for new opportunities and services SMEs, post-investment, to increase their efficiency and profitability.

The hosting company of CBLLTM, Spazi di Impresa s.r.l, coordinates the activities of the Living Lab and provides support to participating stakeholders through local animation and advertising initiatives. Public authorities and agencies that are in charge of territorial marketing also play a role in the Lab's decisions and activities. This Living Lab adopts an open collaboration approach through its cross-boarder initiatives and involvement of 
partner organizations. It has an external strategy aimed at attracting outside investors combined with an internal strategy of continued growth and settlement of past investment within the territory. CBLLTM believes that the freedom to create and being creative is the cornerstone to innovation. More importantly, CBLLTM believes innovation can be increased by sharing the creative work with others allowing external contribution. It is through external improvement and collaborative development that methods, tools, and techniques are advanced to state-of-the-art status.

The living lab involves several stakeholders who have committed to form the core team:

- $21 \mathrm{C}$ Consultancy - A female owned and operated SME

- $\quad$ ANETEL -A non-profit Larnaca District Development Agency representing 4 Municipalities and 48 Community Boards.

- Dialogos, Nea Media-A consultancy company specializing in innovation services and development for SMEs and other Organizations

- eZAVOD - private company for sustainable development. Uses European funds to Research and Develop projects in ecology, energy, economy and e-business.

- Kielce Technology Park - A business incubator for new entrepreneurs. A favorable environment with support for every stage of development.

- Pisa Leaning Lab - Virtual place of aggregation for to work together to generate innovation, develop new products and invent new services. 
Stakeholders involved in the living lab look forward to several benefits. Being part of a collaborative team gives the members the opportunity to network with each other, share their experiences and expertise. Businesses seeking to scale up have an increased chance of success with the added investment from local and foreign projects including European union funding and programs.

To achieve its objectives, the living lab setup databases to enable innovation development. It collects data via survey of local supply and industrial real estate (for rent/sale), conduct analysis through the database and create publications on their website. CRLLTM uses their own developed website to stay in communication with its members and to update the public of its on going work. CRLLTM has to models of financial sustainability. Firstly, it receives funding from public funding form local and national levels such as CIPA and the Planning Bureau. Private organizations such as banks and audit firms also support the living lab through financial means. The world bank, FAO, MED and IPA of the European union also supply funding. CRLLTM second model of financial sustainability is paid services. As a territorial marketing initiative, it offers; training to ANETEL staff (onsite and remote), internet marketing, and promotion of country level database, data collection and publication, database and survey analytics, data management, revision and updates.

\section{Antioquia, Department of Knowledge' Living Lab, Colombia}


Department of Knowledge is a living lab located in Antioquia, Colombia, leading the country in research with $26 \%$ of research projects carried out at the national level. Through a joint, multi-sectorial collaboration strategy, the living lab seeks to harness ICT as a tool for open innovation, to grow new business and strengthen entrepreneurship throughout the country. The objective is to positively transform the country away from violence, corruption and other disasters, to a more prosperous social and economic state. Through social innovation, social inclusion, tourism, cultural services, development of smart region and security, this living lab will improve lives of its citizens and become a pilot model for other developing countries. Currently the living lab is strategically restructuring its Department of Knowledge into nine sub-regions to better manage and develop innovation. It has also taken the initiative to participate in strategic planning of knowledge territory and helping in literacy, education and training in ICT and Science. Its continued activity in research and development is aided with their management of national and international cooperation, media cooperation and financial sustainability.

The lab is composed of companies, unions, universities, research centers, technology parks, regional governments, civic organizations and various institutions. Below is list of all the stakeholders involved by category:

- State institutions at the departmental and municipal levels: Government of Antioquia with its 125 municipalities, City of Medellin, Metropolitan Area Aburrá, Institute for the Development of Antioquia - IDEA, Empresas Públicas de Medellín - EPM. B. 
- Private Sector (Unions and Financial Sector): Medellin Chamber of Commerce, Chamber of Commerce Aburrá Sur, Chamber of Commerce of Eastern Antioquia, among others. $\mathrm{C}$.

- Higher Education Institutions: Universidad de Antioquia, Universidad EAFIT, Universidad Nacional de Colombia- Sede Medellín, "Universidad Pontificia Bolivariana University in Medellin, among others.

- Third Sector Organizations: Territories of Tomorrow Foundation, European Association of Arts, Education and New Technologies - ARENOTECH. I.

- Social control and citizen participation: Citizen Oversight of Medellín, Community Associations, Community Action Committees, Community Housing Boards, Local Administrative Boards, Indigenous Organization of Antioquia OIA, Women Heads of Household, organizations of Black people, Organizations of Displaced Persons, Organizations of Former Rebels, among many others. J.

- Major national institutions that support the Department of Antioquia in Technological Development and Innovation: Administrative Department of Science, Technology and Innovation -Colciencias, Ministry of Education, Ministry of ICT, Ministry of Commerce, Industry and Tourism, Ministry of Agriculture, Ministry of Environment, National Service-Learning SENA.

- Citizens Antioquia

The living lab Antioquia is a joint organization comprising of the state, private sector, civil society organizations and academia. However, the Country Foundation of Knowledge and the Government of Antioquia conduct the coordination of the living lab. 
The Institutional Network of the Department of Antioquia that comprises the governmental organizations, each has their own missions and functions. As such, each strategic decision is made by inclusion of the Directorate of Science, Technology and Innovation of the Government of Antioquia, and the Institute of the Development of Antioquia (IDEA). Open principles of teamwork, common good, cooperation and community involvement are at the heart of the living lab. Following Colombian law, which is consistent with the agreement of the World Trade Organization, the living lab respects member's intellectual properties. A framework of cooperation has been set up to help manage the legal collaboration of the members of the living lab. Through specific agreements in associations with the Country of Knowledge Foundation and the Governor of IDEA, the lab upholds social and specific innovation between the members. The financial sustainability relies solely on the individual members of the lab. As long as each member (who contributes accordingly) is financially autonomous and able, the lab will continue to see growth and prosperity.

The infrastructure required is composed of both hardware and software capabilities. To achieve free wireless connectivity, telecenters, community Internet access points, and mobile telephony, the lab needs to install new broadband technologies of optic fiber connectivity with more bandwidth and mobile connectivity as well as supply an assortment of computers for the department. In addition to hardware, the lab requires free software to operate the ICTs and tools for group management of the living lab operations. Luckily, the members of the lab provide their products and services to help establish the 
necessary infrastructure. Any future technology developed in the lab is also utilized as an asset to contribute to continued growth.

The lab keeps an up-to-date website for all members. It communicates its activities through conferences, lectures, workshops, seminars, talks and social networks. Additionally, the lab utilizes the personal radio stations of the government and the universities to further disseminate advertisements and other information about the labs activities. The government of Antioquia provides, at its discretion the use of its own TV channels for the labs use. Keeping up with modern times, the lab uses live streaming a social media technology such as YouTube to further their array of communication techniques.

Citizens play a key role as users during the research, entrepreneurship, and complete development process of the living lab. Some of the participating institutes help with the labs methodologies by either attracting and organizing users as a pool of volunteers, or actively recruiting users project-by-project basis. Other organizations act as communication coordinators between the users and academia, companies or public agencies, providing a slue of services directly to them or through other partnerships. As a whole, the living lab provides training, workshops and e-learning to empower the users. Conferences, discussions, media (radio, Facebook, twitter, YouTube etc....) and other modes of communication are used to connect the members and allow for communal collaboration. Members of the lab have an opportunity to enhance their marketing mechanisms through the labs activities during the integration of all the participants. 
Moreover, the uses of technology platforms are made available to the participants as well as the studies, research, and training that the lab conducts. Users as well get the benefit of using state-of-the-art technology that has yet to be commercialized. The disadvantaged, the discriminated and oppressed people get the satisfaction of being recognized and included in social innovation.

\section{A.2 BAALL}

Bremen Ambient Assisted Living Lab (BAALL) is a research apartment designed to assist the elderly and the physically/cognitively-impaired individuals. It was developed in cooperation with the University of Bremen, through a European Union funded project for SHARE-it. With a history in mobility assistance, home automation and artificial intelligence such as wheelchairs that are able to safely navigate inside the apartment, the lab is now seeking to develop infrastructures that allow users to upgrade features on demand without major construction or adaptations. Its focus is people's well being and health, Internet of things, energy efficiency and smart cities. BAALL offers the unique opportunity for its members to test and evaluate the technology through user studies in a realistic home environment. The users themselves are granted the chance to experience trail living in the apartment for several day at a time.

DFKI fully finances the lab through national and European research funds. All operational costs including materials and salaries are covered by DFKI. The lab hopes to switch a to paid-service model where they may rent out the lab for research or conduct product evaluation on behalf of external entities. Several German IT companies founded 
DFKI and other research facilitates in 1988 as a non-for-profit public-private partnership. It currently owns BAALL and operates the lab through a secure cognitive system group (a flat organizational hierarchy) that allows for quick and decision making process.

In addition to DFKI's website, which the lab uses for public dissemination of its works, social media such as Twitter, YouTube and Facebook also play a role in the labs communication strategy. Live demonstrations in the lab, conference presentations and expositions are professional channels through which the lab promotes its activities. On occasion, the lab has been featured on TV and in magazines for their innovative work.

Otto Bock, Miele, Pressalit Care, Raumplus, Schramm, and Miditec are a few of the stakeholders that sponsor the lab through their furniture and appliances. However, the network of stakeholders that come together for collaboration are much larger and include DFKI's industrial partners. Such stakeholders are: Stiftung Friedehorst; a care center for seniors, Gewoba; a real estate company in Bremen, Daimler AG, Deutsche Telekom AG, SAP AG, Software AG, Attensity Europe GmbH, Microsoft Deutschland GmbH, Deutsche Post AG, BMW AG Deutsche Messe AG, EADS Astrium GmbH, Intel Corporation and Ricoh Ltd, Semvox GmbH and Schwartz\&Stahl GbR.

The lab has all the necessary technology to operate a realistic living environment for two people. The lab integrates and adapts off-the-shelf technology with software provided by the SME partners such as Semvox and Schwartz\&Sthal to develop the assisted systems in the apartment. Other partners provide necessary household appliances and furniture 
(kitchenette, refrigerator, sliding doors, bed etc....) required to complete the test environment realism. The living lab has also established a 3D model of the test environment to help designers to simulate the deployment of new devices into the apartment. BAALL has linked the virtual environment with the apartment so that the model can be used as a user interface to control the real test environment and be visualized/monitored in virtual reality. Through the current infrastructure, the lab aims to realize the full requirements of the assisted home apartment to then modify the technology so it does not require extraneous effort to install/upgrade in future infrastructures. BAALL believes in utilizing open standards to simplify and promote the integration of third party products into their offerings. As such, all intellectual property concerns are governed by the open licenses for each integrated open source module.

The living lab strives towards a true user-centered research and development approach to implement and evaluate prototypes. By involving the handicapped individuals, the lab is able to learn about their needs and in return present their technological ideas to convey the possible solutions to the users problems. For this reason, the lab does not limit itself to simple observation of users in the test environment, but encourage and emphasize open dialogue between designers and users. To better assist in the process, the lab cooperates with hospitals, care centers, civil associations and public health to pull information (through questionnaires) about user needs and to examine their feedback about the realworld activities and usability of the prototypes.

\section{A.3 BASAKSEHIR LIVING-LAB (BLL), ISTANBUL}


Basaksehir living lab (BLL) is an incorporated company located in Istanbul and is owned and operated by the municipality and Basaksehir Applied Technology \& Innovation Company. The lab hopes to establish a link between municipality, business enterprises, academia, media and citizens to successfully collaborate towards higher standards of society. It wishes to set higher standards when considering the desires of persons and applying urbanization principalities to both public and private users. In other words, the lab hopes to provide unconditioned customer satisfaction to its citizens raising their education and control in their area. By creating both a real-life and online social community, of professionals and non-professionals, the lab will enable idea generation, start-up entrepreneurship and an improvement of the media, healthcare, smart energy and education industries.

BLL currently provides user-experience centers and business incubators to help entrepreneurship. As a platform, the lab connects with other living labs with ENoLL to create a market place for innovators of the ICT based products/services. The lab currently uses facets of ICT technology such as forums to have a meeting of the minds (users and service providers) to discuss ideas, which are complementary to the hosted venues, and knowledge centers that the lab provides. It makes its works public through seminars and events and encourages interested parties to start learning by joining their hobbyist courses. 
BLL provides an environment for promoting development of innovative ICT products. It has currently set-up an outdoor security system with HD street cameras that are connected to police information databases and other municipal resources. BLL also has established a geography information system, which integrates e-map technology with location data on municipal servers to create a location based information service. Moreover, the lab has equipped and furnished facilities such as homes, incubators, and showrooms with innovative products sponsored by participating companies. Other resources required by the lab to continue operations include, call centers, web-based platforms (databases, GSM stations etc....), wireless connectivity, automation tools and knowledge houses.

The management team of BLL controls all the operations and decision-making process. The management team consists of Basaksehir municipality, Orli management consulting, Tikle mobile application \& services, LifeCo Well-being services and ARA research \& business development companies. As a municipally owned living lab, BLL is funded mostly by the municipality who have invested for the past few years laying down the foundations for the living lab foundation. Contribution by partners are supplementary with the lab charging success, and rent fees to business to also help offset the costs.

BLL has grouped its members into four different categories for each to play a role in the innovation process. "Effective people" are the well-known personalities that work in the public and private sector. These members will be used to accelerate developments by promoting the commercialization of products and services. "Effective personalities from 
media" are a group of people who will help educate the public about the activities of the lab and encourage continued and growing participation. "Internet generation children and youngsters" are the group of users who are chosen from the very successful school districts. They will help stimulate innovative ideas as well as provide feedback about current lab offerings. As youngsters, it is expected that they will help build up a user base through word of mouth promotion. "People working with Technology Development or Education" are the executives of R\&D companies or professors are the solution providers. With their help, product value, development work and prestige of the innovations will fuel the living lab. BLL manages its user base on three different fronts, Social Networks (Collaboration), Mobility and Service Focused Architecture (openness and transparency). The lab organizes workshops (in-person and online) to showcase to the users the offerings and then collect relevant feedback.

As a participating member in the living lab, you can expect to receive several benefits. Obtaining guidance and business management support to help propel your start up is one. The use of living lab facilities can aid in testing new technologies with real users as well as establish a market base through the labs events and showcases. Participation alone will guarantee the members logo/brand be included in publicized material and labs advertisements.

\section{A.4 CASALA Living Lab}


The CASALA living lab is a university-founded lab based in Dublan, Ireland. It's array of environments and actors provide an opportunity for a complete development cycle of innovation in the fields of: Energy Efficiency, Sustainable Energy, Climate change, Well Being, Health, Smart Cities, Future Internet, Internet of things, Social Innovation, Social Inclusion, e-Government, e-Participation, Creative Media, User driven contents, Social Networks, Web 2.0 Regional, and territorial and rural development of Smart Regions Security. With the help of care-givers, engineers, scientists and service providers, the living lab will help companies, NGO's and government organizations to design build and test products for improving lives of older people. The Dundalk Institute of Technology, the Dundalk Town council, the Health Services Executive (north east area) and the Sustainable Energy Authority of Ireland (SEAI) are currently the primary stakeholders of the living lab. The Atlantic Philanthropies and Enterprise Ireland provide most significant funding to CASALALL.

The living lab has a steering committee as well as an executive management board that strategically governs CASALA's objectives and makes critical decisions on behalf of the organization. CASALALL structures the management of projects and their associated intellectual property prior to the undertaking of each collaborative initiative through involved discussions and consensus agreements of the participating actors. The agreed upon terms are formulated into a document and signed by all participating actors as legal proof of the guidelines. The lab attempts to operate openly by adopting the best practices in intellectual property management. The lab forms joint ownership management 
agreements to regulate the arrangements between participating members and regulates it by the Institutes Intellectual Property Policy.

CASALALL actively promotes, through integrated community-oriented services and sustainable home designs that incorporate age-friendly technology the benefits of the labs ecosystem and its positive impact on older peoples' quality of life and well-being. They collaborate within County Louth conducting small-scale development and validation with older people to both help business in the sector and further attract new business to the lab. Communication with the public and fellow members is achieved through active web tools and social media outlets such as YouTube, Twitter, SlideShare and Facebook.

The lab attempts to operate openly by adopting the best practices in intellectual property management. The lab forms joint ownership management agreements to regulate the arrangements between participating members and regulates it by the Institutes Intellectual Property Policy.

The lab has sixteen smart aware apartment homes at its disposal for the implantation of new and innovative technology. Fifteen of the apartments are owned and occupied by older people who are actively involved in CASALA's projects. The last apartment is unoccupied and utilized for prototyping, testing and validating new technologies and occasionally as a showroom to demonstrate the labs progress. The apartments are equipped with room presence sensors, contact sensors on doors, electric usage sensors, water and heating usage sensors, light switch activation technology, ambient light \& temperature sensors, and social intruder and fire alarms. Each home is also equipped with 
Internet TV and touch screen mobile devices for home application control. In addition to the physical test environment, the living lab has an immersive virtual reality system that renders a high resolution, 3D, room-sized version of the apartment to help develop and test new architectural or technological concepts before prototyping a physical product.

CASALALL has access to a large network of users (older people and caregivers) that help perform idea generation and product validation. The candidates were not preselected by researchers of the lab but were by the housing authority. As such, the lab followed ethical protocol and informed the users of the undertaken projects and received voluntary consent to participate. The lab uses focus group meeting, one-on-one interviews, and surveys in addition to in-field trials so that the lab can gain insight into the users thoughts and opinions regarding the products and services. Through the Internet powered apartment and custom wiring, the lab is to easily integrate new products and monitor their activity via software. The advanced Internet system with all the sensors gives the researchers the ability to observe positive impact on the users life through the provided technology.

\section{A.5 CIMLAB CaraÄbe Innovation Martinique Living Lab}

CIMLAB is an innovation living lab located in Martinique that focuses on the needs of its citizens and the development of its territory. The objective is to strategically organize key territorial players into a collaborative manner to adapt to the evolution of the 
globalization economy. To achieve this, the lab hopes to develop a discovery/training space where ideas can be picked up by companies and turned into reality for further innovation and growth. CIMLAB is a public-private living lab and is funded via public and private funds including venture capital, seed money and research funding. Partners sponsor physical resources such as offices and loan their personal staff to the lab. Each undertaken project is first financed through other sources to ensure safety of completion and sustainability of the lab.

The lab is structured as a public-private partnership in order to explore new business models that foster innovative and beneficial products and services. As such, CIMLAB is operated by Communaut $\AA$ de Communes du Nord de la Martinique and the Territories of Tomorrow Foundation. The steering committee runs the labs operations with the management best practices based on EURPace association in 1986. It is composed of representatives from each member in CIMLAB and chaired by CCNM. The management team is responsible for the review and acceptance of new members into the lab as well as approving candidates for project management roles. The lab has a project committee, which is responsible for selecting the projects to pursue and assign appropriate managers to oversee the projects.

There are several stakeholders involved in CIMLAB. The two members that are primarily governors are the community of communities of the North Martinique and Territories of Tomorrow Foundation. The University of French West Indies and Guyana (UAG), Regional Parliament, General Council, National Center of Territorial Civil Service 
(CNFPT) and other Private organizations or associations are all stakeholders in CIMLAB.

CIMLAB uses various forms of communication channels to disseminate updates of their activities and share information with their members. CIMLAB has a dedicated page on the CCNM website which allows for collaboration for innovation by the members. Through the use of eGovTUBE, YouTube, and dailymotion, the community can follow the labs events, work, and presentations via social media. CIMLAB is developing mobile applications to have a unique platform presence on mobile phones.

CIMLAB regulates the intellectual property and divides them into two categories, artistic literacy property and industrial property. The labs philosophy is that the partner providing the idea or product retains the IP. The partner, however, grants free right of use of knowledge and exploitation of the IP provided within the framework of the living lab so that other members (users and partners) can participate in the labs projects. In the event of commercialization of products or services developed or tested in the lab, each partner is unrestricted to negotiate the terms of use of their own IP licenses. To reduce most complications regarding IP, the lab tries to use as much Open Source software and hardware as possible including for its communication tools and networks.

The living lab has several test environments in which users can test and validate products. The cities are interconnected with high speed networks and are slowly improving for further and newer communication technology to be power social networks and mobile 
devices. CIMLAB includes all the members into the innovation development process. It aggregates from its citizens, partners and committees different levels of experiences and know-hows to cross-fertilize each field's competencies. CIMLAB always follows the cycle analysis of needs, expectations and expressions of users by using video, interviews and surveys to capture their attitudes and opinions. The data is then passed to engineers to design and construct the product which is then deployed onsite. The users than iterate through the testing of the product until it reaches satisfaction. If the user base is too large and the project timespan is lengthy, the lab will use blogs or other social networking sites to organize and connect with the users during the project cycles.

\section{A.6 City Lab Coventry}

The United Kingdom living lab City Lab Coventry (CLC) is owned by Coventry University and has strong strategic partnership with the City council with the intention to lead a joint venture. The lab aims to establish the city of Coventry as a test-bed, incubation hub and international showcase for innovations in low carbon footprints. To lower their carbon impact and improve the quality of life for its urban citizens, the lab is investing in ICT technology of cloud computing, traffic management control system and other necessary tools to develop low carbon vehicles and sustainable buildings. Funding is achieved from external sources including public grants and private investments.

Currently the lab is investing and implementing digital environment home energy systems to help create sustainable buildings. They have eight road hydrogen fuel cell 
vehicles and filling stations as well as forty-five EV charging points to demonstrate the Low emissions vehicle initiative. The lab complements its infrastructure of technological parks, center buildings and city junctions with human resources of university expertise and partner IPs. Access to real-life test environments supplemented with expertise help speed up research and development as well as taking the product to market.

There are three levels of governance in the CLC: executive committee, operational management group, and theme leads. The executive committee is responsible for the overall activity of the lab and reviews applications for support, make investment decisions, govern intellectual property, engage stakeholders and promote the CLC vision. The executive committee is composed of Coventry University, Coventry City council and is directed by John Lathem. The operational management group is responsible for the day-to-day operations of the CLC. They apply for funding, manage finance, monitor performance, organize events, and manage communication and reports to the executive committee. The theme leads are responsible for the technical delivery of the undertaken projects by the lab. These leads are often individuals appointed to oversee the field of their expertise. The lab encourages the use of and continued innovation in open source. To optimally exercise creativity, collaboration and innovation of the living lab, CLC has a policy to own and share intellectual property with the whole consortium of members. If a private member owns the IP, the lab encourages them to make it available and open under creative common terms. Nonetheless, each project undergoes IPR reviews before commencement and a formal agreement is drafted and signed to ensure satisfactory IP control. 
There are several stakeholders involved in the CLC living lab:

- User Categories:

- Citizens of Coventry, including students and graduates

- SME's internationally (COVUNI typically work with 7,000 SME's internationally per year)

○ Research community

- Public organizations and NGO's

○ Large industrial corporations

- Networks:

- The Motor Industry Research Association (MIRA);

○ National Physical Laboratory (NPL);

○ IBM;

$\circ \quad 02$,

○ British Telecom;

○ Severn Trent Water;

○ Jaguar Landrover; EoN;

○ Cisco, ARUP; Transport Research Laboratory (TRL);

○ Bright Automotive; Unipart Manufacturing Group and Microcab Industries Ltd.

CLC involves the users from the beginning of the idea generation process and through the live test of products to the final commercialization of the product. The lab uses a 
collection of tools and methods to engage users and connect functional innovation with user behavior to better the design of the products. Online forums and other social media networking are the primary form of communication of the lab. However, they do present at conferences, co-author articles and engage in international collaborative tasks to make a presence and demonstrate their work. Communication between its users can often to the form of face-to-face consultation, audiovisual telegraphy and the physical use of the test environments. There are several benefits to participating with CLC. One, an SME may get financial aid to help drive research and development in a user-center environment. Public relation, promotions and business staff can also be assigned to help with business activities and help advance collaborations and sponsorship. The lab also offers incubation space where entrepreneurs get access to desk space and advisors to support in legal, financial, cultural and practical issues that the business might face. For the citizens, this is an opportunity to receive methods to reduce their energy consumption, gain a sense of pride for the city and experience economic growth with the potential of new job availabilities.

\section{A.7 Cretan and South Aegean Living Lab}

The Creatan and South Aegean living lab (CSALL) is a living lab located in Greece that was founded as a partnership by the University of Crete that has been developing software and applications for several municipalities. CSALL is hosted and coordinated by the University of Crete, which is supported by the computer center's permanent staff. 
Using European Union funding, the lab is position to work on programs for egovernment, education, networks, and multimedia lab and software development. The objective is to design the various modules and programs in an open fashion to allow for interconnectivity with other applications for better customization per user needs. CSALL uses modules and tools that have been developed under open source licenses. Their philosophy is continued use and development of open source tools to allow for the free exchange of products, software, and data between the partners. The lab receives its funding from various European Union programs focused on researching e-government services and technology. In addition to the EU, the lab receives financial support from the national government to help pay for operations. Although the participation from businesses does not have to commit funds, projects are co-funded and sponsored by the SMEs and corporations.

There are several stakeholders involved each playing a role within the lab. University of the Mountains creates contact with residents and local partners to build up a community network. The Technical University of Crete supplied five different engineering departments for the purpose of research and development. Technological and Educational Institute of Crete provide technological education and training for innovation research programs and help disseminate it to production sectors. The Municipality of Heraklion is the test bed city for the new products and services to implement for its citizens. Ikaros network and Heraklion Chamber of commerce and industry carry out surveys to gain valuable feedback from citizens and to promote participation of businesses. Other 
partners include the Region of South Aegean, Forthnet and SME's. All the partners have agreed to collaborate and contribute to the activities and objectives of the living lab.

Members can expect several advantages of being part of the lab. As a member of the lab, innovative collaboration can be conducted under an operational framework. SMEs get the opportunity to work with academia and government to access methods and funds and test their products with a large number of people to quickly and further advance their products. Users (the citizens) also enjoy the benefits by embracing new ideas and products that help improve their conditions of their lives and local market.

To date, CSALL has developed much e-government programs with networked e-services that allow citizens to interact with their local government and the rest of the community. The lab uses different features of web portals to expose their population to the innovative products and services developed such as e-government and e-transactions that can also be found on now on mobile devices. CSALL has at its disposal fifteen e-government (municipal) web portals that house informational material, applications, forums, polls, and several electronic features for efficiency optimizations. The web portals allow for communication between citizens and government, expressing opinions on public matters and legal transactions to be completed remotely. In addition to the web portals, the University of Crete hosts for the lab the management software and framework to help the innovation processes. 
CSALL works with academia and SMEs to help create innovative products for the end users. Development occurs in the facilities of the universities and SMEs. They have developed platforms for mobile and Internet connected devices to help facilitate the interconnectivity between citizens and local governments. The government provides access to a large number of end-users and Internet hot spots for access of digital functions and data on tourists, visitors, and citizens. Through forums, pools and the mobile platforms, users can express their opinions of the innovative products and provide necessary feedback to complete development.

\section{A.8 E-zavod Living Lab}

The Slovenian living lab E-zavod is a private non-profit organization that focuses on supporting sustainable development of ecology, energy, economy, e-commerce and ICT technologies. The lab plays a role in national strategies offering supportive activities for the operational plans of Slovenian ministries and the implementation of EU programs. Employing nine experts with rich experience in project management of the focused fields, E-zavod works towards socio-technical changes to enhance the territories energy efficiency and waste disposal programs and remodel future environmental activities of the area. E-zavod uses simple methods and tools to conduct its user-centric research and development. It starts with users and organizes seminars and or workshops to brainstorm ideas for both problems and solutions, and then sets project managers to head the projects 
activities including raising capital. The produced innovation is then implemented and tested wishing the local area for validation before commercialization.

The lab uses websites, promotional videos, scholarly and magazine articles as well as workshops and conferences to communicate their activities, objectives and progress. The website is the primary portal through which public recognition is achieved as news is posted to update of the progress and effectiveness of the projects. The introduced workshops to achieve two-way communication with the public to learn of the perceptions of the problems that need to be solved and network groups together for better collaboration.

The living lab is currently funded through public means but it has the intention of adopting a private business model to become a financially sustainable entity. E-zavod wishes to offer project management related services to external actors who wish to use the labs resources and conduct product development through them. It can prepare documentation for European Union funds, conduct feasibility studies, prepare costbenefit analysis, and environment assessments for sustainability projects. As such, the lab can generate funds through consultation services and lobbying procedures. The living lab can also provide incubation space for SMEs to generate funds. As a private non-profit organization, all funds generated must be based the successfulness of the involved companies. The business model for this living lab acts as a metric that demonstrates the follow through of products and services generated through the living lab. 
E-zavod uses a simple organizational structure for quick and easy decision making. It consists of a single director, who is advised by two counselors, who operationally directs the five various project managers of the different activities of the lab. The project managers act as the leaders for the activities responsible for the administrative and communication roles to effectively direct the project.

E-zavod has accumulated several participants to create a collision for the living lab it includes: Municipalities, Chambers of commerce, Ministries - Non-governmental Organizations, Regional Authorities, Research Consortiums, Technology Transfer Centers, SME's and others.

E-zavod needs the full infrastructure of ICT and accompanying software to successfully implement the renewable and sustainable forms of energy and waste management. Many benefits exist for those who wish to use the living lab. Entrepreneurs and SMEs have available (through the lab) a business incubator with physical desk space and complementary consulting services. The lab also grants businesses access research facilities and international markets through its networked collaborative partners. Even users benefits through the innovative products produced especially energy management tools that will help save them money and create a cleaner environment.

\section{A.9 ErgoLab, Humanizing Information Systems}


ErgoLab is a living lab located in Saint Sabastian, Spain. The lab's goal is to address user and corporate needs involved with Information Systems. ErgoLab seeks to become a leader in optimization services of resources linked to ergonomics, products and infrastructure. Working with the Basque Country Government, and the Department of Health and Industry, the lab has created a collaboration to analyze the views and needs of the target group in relation to the real-life interaction of information systems and healthcare. The labs is owned and operated by GAIA, a Basque's electronic, IT and telecommunication industry association/cluster. However, the lab still engages other institutions such as companies, research centers, other living labs and Universities. ErgoLabs partners include: Tecnalia Research \& Innovation Educational entity, Universidad de Deusto Regional Administration, Basque Government, GAIA and all its enterprises, citizens, and the provincial council of Bizkaia. Each stakeholder plays a role in the lab:

- GAIA: supplies the infrastructure (including equipment), ICT consultation, system integration and the implementation of business solutions. They are the actors who provide technological solutions to the discovered needs of users through their research and development divisions.

- Government of Basque Country: Provide funding to support the activities of the ErgoLab. Additionally, the set the laws that govern the actions of the lab to protect and represent the public and other users.

- Citizens: are the users who test the products. 
Three-tier committee consisting of a Management board, Advisory board and an Institutional board governs the lab. A technical management team carries out strategic planning for the lab and leads the services offered. The project management team is responsible for the resources of the lab by undertaking feasibility assessments, project estimates and administrative roles of the project.

ErgoLab believes in the power of social support and collaborative efforts. It believes that it is the societies responsibility to diagnose its own problems, vulnerabilities, and risks and generate knowledge and solutions on their own behalf. The lab upholds that "social intelligence" is the most important asset representing all relationships and networks a group can have as an ability to solve their own problems. As such, the lab adopts and fosters an open concept accepting new companies, associations, regional entities and others who will collaborate together to create synergies in innovation research process.

ErgoLab carries out usability consultancy services to support the Basque Government and other associations as well as students. Its activities include research on best practices of User-driven Open Innovation and User-Centered Design. As members of ENoLL, ErgoLab hopes to contribute to the thematic domains of eParticpaation and Media network. Through their activities, ErgoLab expects to learn more about "User Experience Behavior" and the associated "Models and Metrics". They will be able to contribute knowledge regarding emerging services and ease of use of information systems. 
ErgoLabs builds its financials on two models, public and service funding. The participating actors such as the government can financially support the lab, however, the labs itself offers several services (such as consultation) to both society and organizations that generates income for ErgoLab.

The lab uses a series of technologies to conduct their user-centered research. Specific software helps with monitoring but it is outdated and needs updating. Eye tracking technology helps monitor user behavior during the use of the products. The remainder of the required technology is standard ICTs, computers, smartphones and mobile devices. The lab engages all actors, from citizens and SMEs to researchers and politicians, in the full development cycle. All of them work with synergy to create a product that is optimized for users and is state-of-the-art ergonomics. ErgoLab acts as a leader in user communities encouraging social intelligence, and influencing their behaviour to adopting a participative attitude towards sustainability and Green IT. The lab collects the submitted problems and presents them to GAIA. The cluster of IT companies find solutions to meet the needs through their R\&D departments. The research teams are split into two teams. One team focuses on the usability, interaction design, information design, web analytics and overall functionality. The second team works on the human factor incorporating psychology, anthropology and sociology to integrate with humanity into the innovation. Once the product is prototyped, it is tested and validated with the users. The marketing team then promotes and pushes the product out to market. The marketing team nurtures the relations between actors in the network to build a critical mass for commercialization. 


\section{A.10 Espaitec Science and Technology Park Living Lab}

Espaitec, Science and Technology park Living lab (e'livinglab) is a living lab located in Castlelon, Spain. Phase 1 of this lab is a "proof of concept" establishing, to the government, companies and investors, the effectiveness of the living lab as a tool for innovation and production. Phase 1 limits the lab to the University Campus, consisting of fifteen thousand students and two thousand researchers as potential users. Later, e'living lab will extend to the whole Castellon city and later to the province to expand the living lab reach into smart cities. The lab was created to facilitate the collaborative work between all actors, not just companies and clients. The objective of the lab is to improve the quality of life for the citizens while growing the socio-economic wealth of the region. It does this by providing its actors various developments, to their STP to design, for development and testing of their products in real environments. It is meant to accelerate the growth of business and entrepreneurship.

The lab has undertaken small projects to test their smart city products. On their university campus, the lab has designed control systems to help improve living quality. It has installed humidity sensors to help monitor and control the consumption of water in the gardens. High-speed legionella detectors have been placed in swimming pools and water supplies to assure no outbreak of legionella. The library is now managed with RFID to detect the in-and-out of books. Temperatures are now controlled automatically for the server rooms. Integrated Solar and Wind power generators funnel energy into lighting 
and charging systems to help power users technology such as mobile phones and laptops. They designed an irrigation control system and a POS payment system to process student enrolment. Lastly, the lab has also implemented a 3D generating virtual environment for students to produce virtual media and products.

A shared collective of the participating actors, the University, involved companies and research centers, undertakes all decisions and behalf of the e' Living Lab. The lab's intellectual property rights is managed and upheld by the university; its perspective is, as the main client and sponsor of the lab, the university sees fit to manage the IPR of prototypes developed by companies for the living lab. The lab uses social media tools such as twitter and Facebook to interact with its users, updating on projects and learning from their feedback. Other forms of communication take on the medium of TV channels, radio shows and web portals. On an academic level, the lab hosts case studies at conferences and publishes articles.

There are several stakeholders involved in the living lab pooling together R\&D groups, companies and government to form a synergy for local development. The primary stakeholder is the Science and Technology Park that founded and owns the living lab. The investors are University Jaume I of Castellon, Business Angels, Ministry of Science and Innovation Industry and Education Councils. Partners who participate in the lab, forming small and big companies, include: Biotica, Cognative Robots, Cite-Trade, XOP, 4TIC, Advanced Wireless Dynamics, and Ingesom. These partners act as sponsors and 
users in addition to other users such as project technical officers of the university and library university users.

From a stakeholder's perspective, the lab offers an opportunity to generate business by means of co-creation. All partners experience a cross-pollination of ideas to help each actor in the generation of innovative products. Additionally, it is a means to help colaunch products for an increased probability of success during commercialization. It presents a mechanism to the Science and Technology Park to generate wealth within the region.

The lab sets forth collaborative roles among actors of the living lab. Through their specially designed web platform "e'fridge", the lab connects the actors together where they can submit their ideas and vote for the ideas to be pursued. The companies with the necessary resources are tasked with the design of the innovative product based on the requirements outlined by the clients and users of the living lab. Advanced webcams and media technology is used to help track and data collect during the testing and implementation cycle of the innovative product.

e'Living Lab is currently financially sustained through public investments from the government and the University. Its future model aims to obtain funding through the output of the living lab as commercialized products and services.

The infrastructure is comprised of the physical location of the university and the installed sensors powered by ICT and software to simulate as miniature smart city. It requires $1 \mathrm{~GB}$ 
high-speed network to ease the load of data traffic between the interconnected modules and actors.

\section{A.11 Flemish Living Lab Platform}

The Flemish Living Lab (FleLLaP) is located in Mechelen, Belgium. It acts as a platform to promote and accelerate the production of innovative information, communication and entertainment (ICE) products and services. This platform works by reducing the transaction costs encountered for collaboration and setting joint operating rules for participants. The living lab platform incorporates infrastructures, users, and various services. Through this platform, the lab seeks to enhance the economy of the region and abroad. The lab provides support in three categories: Smart Grids, Smart Media, and Smart Cities. The goal of smart grids is the sustainable supply and growth (technical and economic) of electrical energy. The lab to test out intelligent homes for the smart grid projects provides products, services and infrastructure. An assortment of media enabling technology (digital TV, internet, tablets and a cloud system) is provided of the smart media program and the testing of media application and services. The undertaken project of smart cities is targeting the local community (residents, businesses, government and associations) to build a social network. The intent is to bridge the groups into an easy and effective communication dynamic to facilitate innovation collaboration.

As the hosting organization of the living lab, Telenet acts as a coordinating partner directing the actions and goals of the lab. However, to effectively manage the lab, they 
have set up a management organization consisting of a Customer Committee, a Steering Committee, and a Platform management team and platform manager. The customer committee is responsible for promoting the lab and proposes to the steering committee and platform manager actions that could benefit the lab. This committee is comprised of representatives of representatives that connect regularly with stakeholders. The steering committee is responsible for various operations including: review of performance indicators, approving changes to work packages, the work plan and resource budget, review and approval of external communications, approval of the membership decisions, and resolving escalations from other groups. The steering committee is composed of representatives from the participating members of the living lab. The platform management team, which is headed by the platform manager, also consists of representatives of the participating members. The platform manager's duties are the daily operations of the living lab. Additionally, the team consists of infrastructure and panel managers who are responsible for the maintenance of the labs infrastructure and test user panel. They report to the steering committee.

The lab uses a public website to host brainstorming activities to generate ideas. The website is also used as a management system allowing users to appraise the ideas that are most valuable. The lab maintains a face-to-face interaction through meetings, events and demonstration sessions to communicate information that is otherwise difficult to post online. 
FleLLaP does not discriminate against private or public organizations or associations that wish to conduct user-centered research. It upholds an open innovation culture that supports diverse collaboration. As such, the members participating often make some of their background IPR available to the lab to further facilitate its build and innovation. The output innovations from such collaboration are licensed to each member under fair and reasonable market conditions. The specifications of such IPR policy are outlined in the consortium agreement. This agreement, which all members must sign, also outlines that data and knowledge produced will remain anonymous and made available as a resource for the living lab platform.

The member stakeholders that comprise the consortium are large companies, SMEs, research organizations, and interface organization. Some of the more notable entities are:

- Telenet: a leading provider of media and telecommunication services

- Alcatel - Lucent: global leader in mobile, fixed, IP and optics technologies and pioneer in ICT applications and services.

- Andorome: innovative Belgian ICT-SME that offers software solutions

- Fifthplay: internet-based service platform that offers to deliver care, comfort and energy services to smart residences

- IBBT -iLab.o: Interdisciplinary institute for Broadband technology

The Flemish government under the O\&O program that aims to make advances in Innovation through the use of science and technology financially funds the living lab. 
The lab uses a generic smart home environment that has been installed for in households of participating test users. There are two hundred and fifty households. The lab has provided fast Internet connections, hotspots, digital TV, wireless modems with wifi, software to connect smart devices and support systems to gather test feedback. The lab utilizes a server farm with open interface software with touch screen displays to power the backend portion of the experiments.

FleLLaP undertakes several activities as part of its platform portfolio. It is responsible for bringing expert participants together and selecting, recruiting, preparing and monitoring the test users. It is responsible for implementing the test products or services into the test environments and conducting the research (both gathering and analyzing the feedback). The lab offers technical support for solving operational problems for the before and after phases of the products test. The members themselves conduct the actual innovation design. The lab can manage the equipment and offer smart money concepts to help the members. The members gain invaluable advantages from the lab. The members get to access a large market group of test users as well as access to network technology and business partners. Most importantly, the innovators get support from the lab to identify problems, run validation and complete the realizations of their products. The labs grants previous knowledge as well to help expedite the testing phases.

The lab first hosts idea jams on the website to solicit user inputs towards problems and product feedback, building up a collage of concepts and ideas. Sessions are held with potential partners that would like to use the labs resources and services and are aligned 
with user feedback to start a research project. The lab sets up in depth profiles for the test users which links the to activity domains (research categories) to help with the collection and analytics of the data during the research cycle. The labs then uses a "prelaunch panel" of two thousand people to validate result prior to the official commercialization process. 


\section{A.12 Future Classroom Lab}

Future Classroom Lab (FCL) was originally founded in 1998 as a "network of networks" prior to the realization of the living lab platform. It was established as a means to further develop, demonstrate and showcase scenarios for future techniques in teaching and learning as a result of the role technology plays in today's society. The lab gives policy makers an opportunity to research and understand the issues relating to technology in the classroom. As a facility, FCL offers open and configurable space, and designs and validates pedagogical activities that demonstrates the use of innovative furniture and other technologies in the flexible learning methods of the future helping teachers and policy makers rethink education for the $21^{\text {st }}$ century. The lab carries out pilot activities for the purposes of testing and validating new and emerging technologies developed in TEL (Technology enhanced Learning) programs. It also attempts to maintain the results. It also showcases learning scenarios that now emerging from iTEC. The lab works to push for the mainstreaming of the new techniques.

The Ministry of education governs the lab. They comprise the decision-making body (the steering committee) and the board of directors.

FCL keeps its doors open to new members associated with educational authorities a number of which are already supporting various Schoolnet as partners in large scale partners already funded by the European Commission. The lab uses social media such as Twitter, Facebook, and LinkedIn as well as websites to maintain an open communication 
channel with its ministries. It also holds demonstrations of its technologies and programs that showcase best practices, large-scale validation results and the ICT tools. The lab releases monthly newsletters to highlight stories.

The lab is financially supported through government funding from the thirty main Educational Ministries who in turn are supported by the European Commission. There are annual financial contributions from industry sponsors and model in place to independently sustain its activities through courses and workshops to teachers and policy makers.

Several companies who provide equipment for the Classroom sponsor the lab, these companies include: Acer, Fourier, Microsoft, Panasonic, Planet PC, Promethean, RM Education, And SMART Technologies. The key stakeholders of FCL include thirty Ministries of Education and the end users who themselves are teachers; ICT advisors and policy makers who all help co-develop the products and scenarios.

The FCL uses an interactive classroom that is reconfigurable and divided into five learning zones each with stat-of-the-art technology supplied by the vendors. The classroom is used as a test and demonstration tool for the promotion of new interactive teaching technology. This space includes video streaming, multimedia, interactive whiteboards, netbooks and tablets. The users (i.e. teachers) receive professional development opportunities, from the lab, directly related to their needs as well as 
workshops, seminars, webinars and professional development activities to educate them on upcoming technology and its usage.

\section{A.13 Future Logistics Living Lab}

Future Logistics Living lab, hosted by NICTA, is the first living lab that operates out of Alexandria, Australia. It is the first organization in the country that provides a platform for business, research centers and government to come together and collaborate on "real world" problems and help build innovative solutions for the future. Its primary goal is to improve the industrial and logistical development of the country, but, In response to rising fuel costs, road congestion and safety risk, the labs would also focus on green transportation, Internet of things, and smart cities. Its objective is to utilize ICT and its advantages in managing information to achieve its goals. The lab requires state-of-the-art technology demonstration facility, factory, a distribution center (warehouse) and access to the four modes of transportation (rail, sea, air and road). Complementary to the basic infrastructure, all other technology is off the shelf technologies with futuristic prototyped features. It consists of RFID \& Barcode label printers/scanners, model truck with live demonstrations of over height collision prevention, voice-picking technology, eco-driver software, mapping/location based services, cloud computing, supply chain management tools and more.

The lab uses a website as the first point of contact for communication between its members and the general public. All news and other forms of media content are 
published to this site. Press releases and tours are also used to raise awareness and show case technologies and achievements.

A committee consisting of representatives from NICTA, SAP and Fraunhofer is responsible for coordinating the activities of the lab. The committee determines which projects to undertake, which technologies are demonstrated within lab space and what information is shared with the public on the website. As the host, NICTA, has supreme governance over the use of the facilities and governs all related health and safety issues. A specific detail of governing is agreed upon in the Partner agreement that was signed by the founding partners. The labs philosophy is fair and open collaboration providing unimpeded access to any individual organization seeking to participate. The intellectual property rights of each project will be managed on case-by-case bases and upon agreement of the involved participants. The lab will not force any IPR regulations but recommends that ideas be protect by confidentiality agreements, background IP ownership remains with provider, IP of output be owned by its creator, and that participants provide each other with licenses for the use and further development of the products.

A network of companies, research organizations and IT, drives providers the lab. Currently, NICTA, SAP, Fraunhofer, Casella Wines, Ericsson, Gamma Solutions, Google, GS1 Australia, Hamburg Sud, Linfox, Tradegate, XAct Solutions, Victoria University, and the University of New South Wales are all participants in the lab. Government and other organizations are welcomed to contribute and join the lab. 
The lab is currently funded through Fraunhofer Project Center and support from the state government provided by Industry \& Investment NSW. In the future, the lab aims to gain financial support from the participants by creating a membership-funding model to offset the cost of operations. Because the goals of the lab is to create national wealth, economic growth and improve the environment, the lab sees it fit to generate and use the external funds from government towards projects that will meet those objectives.

\section{A.14 HACERIA, ZAWP BILBAO}

ZAWP living lab is a non-profit cultural organization hosted in Bilbao, Basque Country. It aims to promote economic and social regeneration through an artistic, innovative and creative urban transformation that should attract public to the region. To revitalize the Rubera de Deusto-Zorrotzaurre district, the lab is turning an old and degraded industrial area into a space where participants can freely express themselves and share experiences of different disciplines. ZAWP believes corporations will get involved in such a lab to demonstrate their social responsibility and commit to their environment by building up spaces that are livable and optimize economic and cultural resources. The lab itself directly manages the mission of the lab.

The lab try's to engage everyone in its activities either as spectators, participants or promoters. However it grants independence to the project promoter to pursue their vision. The projects are supervised and approved by the public entities that govern the lab and 
the public spaces. The lab validates the outputs through independent entities' that have no stake in the operations of the lab.

ZWAP undertakes several activities. It identifies abandoned industrial spaces for reuse and the reviving of the neighborhood. It promotes artistic interventions that encourages and empowers emerging artists. The lab seeks to preserve and conserve the historical areas of Ribera de Deusto and Zorrotzaurre in context of their town. ZWAP also collaborates with municipal government to co-host different events and activities.

ZWAP uses websites and social media to form a two-way communication channel. The lab also hosts seminars and virtual tours to educate and demonstrate their work. The lab has utilized direct marketing, press releases, radio and television to further push out information about the labs events and activities.

The lab involves the Country council of Bizkaia, Basque government, Ministry of culture of the Spanish Government and private companies in their consortium.

ZWAP is financially supported through external funding from government and private entities.

ZWAP requires $\mathrm{W} 3 \mathrm{C}$ web standards to operate its website, and a QR-code software and hardware in addition to mobile networks and a server farm. The lab has eight spaces with a total space of $3700 \mathrm{~m}^{\wedge} 2$ that it uses for creation and exhibitions. Each space serves a different function as follows: 
- LAB 01: a multifunctional space that functions as a space for exhibitions of products they wish to rent

- LAB 02: A restored building that is an incubator for six cultural projects the lab has undertaken

- LAB 03: An initiative for artists to showcase their work.

- LAB 04: Space hosting art, and innovation in need of studio space

- LAB 05: A Company (set up by young people) that develops 3D animation and video games

- LAB 06: The information center for the lab and a retail space to sell art

- LAB 07: A building to be restored to host youth organization dedicated to counseling in entrepreneurship

- LAB 08: An exhibition space for grander events

\section{A.15 Hermes Living Lab}

Hermes living lab is a private company developed with and public and private clusters and institutional entities located in the Madrid, Spain. Their prior objective was oriented towards cloud computing with multichannel solutions for health sector adapted specifically for mobile solutions. Their current goal is to now grow a community and test bed to better establish a living lab platform. Using models of open innovation, the Hermes lab seeks to improve innovation capacity by acquiring expertise of biomedical 
professions, caregivers and users to better understand and support patients in the process of overcoming disease.

Below are the stakeholders as outlined by the lab in their category of membership (Figure 19).

\begin{tabular}{|l|l|}
\hline Owner & Centro de Imagen y Tecnología del Conocimiento Biomédico (CITEC -B, S.L.). \\
\hline Investor & Madrid Network Clúster de Salud y Bienestar \\
\hline Partners & $\begin{array}{l}\text { NEC } \\
\text { Philips Salud } \\
\text { Xeron Healthcare Corporation }\end{array}$ \\
\hline Users & $\begin{array}{l}\text { Centro de Cirugía de Mínima Invasión. Dr. Jesús Usón } \\
\text { Agencia Lain Entralgo } \\
\text { Hospital Universitario Fundación Alcorcon } \\
\text { Hospital Universitario La Paz } \\
\text { Facultad de Medicina de la Universidad Autónoma de Madrid. Decano Dr. Juan Antonio Vargas } \\
\text { Facultad de Medicina de la Universidad Complutense de Madrid. Dr. Ramón Merida Velasco } \\
\text { I.U.C.E. de la Universidad de Salamanca. Dr. Juan Antonio de Juanes } \\
\text { Santander Hospitales (Banco Santander) } \\
\text { Asociación Madrileña de Anticoagulados }\end{array}$ \\
\hline
\end{tabular}

Figure 19 Stakeholders of Hermes Living Lab (ENoLL, 2014)

The organizational structure of the lab consists of a management committee, a technical committee, a coordinator, an office of management and a scientific technical advisory board. The management committee is responsible for the decision making process involving the undertaken activities of the lab. They are tasked with the duties of approving projects and associated budgets, acceptance and termination of members, appoint coordinators, and settle disputes between parties. They are also responsible with ensuring good communication between members. The management committee only consists of five voting representatives but may add new members by unanimous 
agreements. The technical committee's role is to ensure that all work conducted meets the requirements of the project details and when necessary, detect and correct deviations. This committee prepares and signs off on technical and financial reports on behalf of each project. They advise the management committee about control of project results in the event of IPR strategies. The technical committee is chaired by the coordinator and its constitute members are leaders of the undertaken projects. The coordinator, who heads the technical committee, acts as correspondent between the living lab and the CITEC. That role guarantees the compliance of the technical, legal and administrative and financial variables in the Lab and monitors the activities to ensure such compliance. Lastly, the advisory board is a team composed of research centers and medical representatives who use their expertise to guide the decisions made by the committees. Hermes lab develops tools and training programs through its virtual 3D reality and established cloud computing to provide solutions to the members such as patients, family, caregivers, researchers, doctors and more.

Hermes is committed to the use of open innovation models and incorporates and offers free software so that members can operate without restriction. The members of the lab are informed and have agreed to operate under the labs IPR policy. That is to say, they have agreed to:

- Inform the lab of "essential IPR" as soon as they become aware that it is related to the prepared or published specifications. Such information is made available to all members and non-members. Note, none are obligated to conduct and IP search. 
- "Essential IPR" is defined and made available under license as agreed to in the lab's application form.

- Non-members are not obligated to provide IPR documentation, but members must follow the lab's IPR policy

- The lab does not make or guarantee the licensing of IPR, individual members must seek to resolve such issues independently.

The Hermes lab seeks to become financially sustainable under a multisided business model that offers the use of its developed 3D models (Virtual City) for revenue generating services. The deployment of these solutions provides customized augmented realities to different buildings and structures that various members can pay to simulate their requirements in.

The lab requires is augmented $3 \mathrm{D}$ technology in conjunction with a cloud infrastructure and an Elgg instance. These technologies together support the Hermes social network that is capable of running the necessary activities of the lab. Hermes living lab has oriented their methodology on the use of virtual environments to eliminate the requirement of physical space. This method allows the integration of all members over Internet to share their opinions, and test their solutions in their own comfort zone. With free access to the services, Hermes collects and stores all data from the users, which currently is over two thousand. Contributions made by users and the lab through monetary means rewards members or time limited access to free services. 


\section{A.16 JF OCEANS}

JF OCEANS is a private company living lab located in Belgium and is connected to the applied research TELIN associated with the University of Ghent. Their focus is in codesigning the improvement of customer convenience and experience. They aim to contribute to ENoLL knowledge in the domains of Future Internet, Internet of things, Industrial \& Logistic development and security. The lab evolves these products in a virtual environment with citizens and corporations. JF OCEANS organizes its resources to better suit the many retail sectors dedicated to the innovations of the lab and encourages the exchange of ideas of between the various types of business. The lab creates economic value and industrialization aiding the economic growth of Belgium.

Despite being a private company, the labs believes in the usage of open source technology, as it is a means to enable collaborative in the co-creation accelerate innovation on public usage. The JF OCEANS serves a private form of IPR principles. Because all the projects are unique, the lab deals with each project individually but generally protects the members with confidential and non-discloser agreements. However, the lab is working on building an IPR framework that is more promising. JF OCEANS collaborates with a growing a network of academic research centers and other retailors.

As a private company, JF OCEANS set up a four-person team to manage the living lab. Alain Van Buuyten has masters in econometrics, MBA and has 15 years of management. 
Philp Van Gasse has masters in applied economics, MBA and 10 years of management in consulting of supply chain and management of transformation of business. Jurgen Dekeyser has a university degree in IT, solution engineering, more than 10 years of experience in solution design and development and user testing. Filip Davids also has a university degree in IT, innovation architect, with over 15 years in Internet business

JF OCEANS promotes its activities and company through its website and personal dedicated virtual spaces. They have presented at conferences and co-authored articles with other living labs to legitimize their position in the professional industry. The have also implemented the use of social media to the best of their ability to connect with their users.

JF OCEANS is financially sustained through private funding. It uses a private business model with an open platform to offer a cost-effective co-creation process. Additionally, the living lab makes money through custom games and their deployed web based environments. JF OCEANS also receives public funding to help accelerate the labs deployment of its innovation platform.

JF OCEANS has supplied their own infrastructure necessary to conduct their living lab activities. It consists of a fully augmented 3D test bed that enables a mixed reality interaction. They use digital cameras connected to Internet and the cloud for applied motion analysis. It is situated in a $500 \mathrm{~m}^{\wedge} 2$ area with installed remote controls, video streaming, augmented reality boards, sensorology environment and tracking sensors. 
These technologies allows for testing smart flow and motion, which is valuable for the innovation of motion analysis and its translation into real-life environments. JF

OCEANS basically uses such infrastructure to understand human interaction and motion in real time and real life contexts to develop business intelligence.

JF OCEANS uses focus groups, scenario building, story telling and surveys to extract from the users the sticky information of their needs and perceptions. They then utilize its fully augmented virtual reality to create test environments where users can be monitored to validate specific business conceptions of customer and employee experiences. These behaviors are monitored and recorded unto a back end server for future analysis. The lab has connected multiple devices over the Internet to simplify the use of technology and data collection. The lab uses gamification techniques to promote and solicit behavior from the user to contribute their knowledge and time to co-developing the technology with them. The participating members of JF OCEANS get to use a framework of consulting experts that manage the process of defining the project, conducting the experiment, and industrializing it on behalf of the investor.

\section{A.17 Kielce Technology Park Living Lab}

The Kielce Technology Park Living Lab (KTPLab) is a public venture implemented in 2008 that is hosted by the Municipality. Despite it being a pubic-private partnership, the lab is a budgetary unit that is $100 \%$ owned by the Kielce Municipality. Its mission is to fuel the economic activity in the region, raising the level of innovation and 
competitiveness in the region. By transforming Central Europe into a laboratory for innovation, KTPLab will become a key actor to expand the social, organizational and technological dimensions of innovation. The lab will draw on the cultural heritage to strengthen cohesion and promote collaboration. It intends to supply design technology, 3D prototyping, methods and IT systems to develop a smart specialization platform. The director who has been granted power of attorney governs KTPLab in alliance with the Mayor and City Council of Kielce. The detailed decision process is conducted through coordinators in a steering committee that is composed by representatives of the partners. The lab pushes to create a friendly, innovative, environment allowing for various actors such as NGOs, Users and Universities to work together to develop innovate products and services.

KTPLab believes in open innovation and in the power of innovative collaboration. As such, the lab invests great effort into building and nurturing relations among members to form a cluster of public and private teams that can cooperate towards scientific and entrepreneurial goals. The lab has structured its openness on the Finnish model where it is science and industry that work together to produce tangible objects of value for scientific institutions, business and society. However, the lab does not apply special IPR rules regarding such open innovation but simply follows the countries set laws. Each member is allowed to protect their idea, artistic work, trademarkible symbols or phrases and patenting their innovation. The lab will take the necessary precautions in protecting records, information and results of the labs work as to not break the law. 
The primary stakeholders have been the municipal government, innovation offices and universities that support entrepreneurship. However, the lab does have partners at many different levels that could be considered stakeholders. At a national level, the Polish Agency for Enterprise Development, The Agency for Foreign Investment and the National Center for Research and Development. The local level consists of the municipal government, the surrounding universities, NGOs, and career offices. These include, City Hall, University of Technology, Jan Kochanowski University, Cultural center, Museum of Toys and Play, Marshal Office and Innovation office, Technology Transfer Center, Chamber of Industry and Commerce, School of Economics and Design Center. KTPLab is financially supported using European Union funds. However, the lab is seeking to gain funding from participating members in the future. Additionally, the output innovation products of the living lab will be sold to generate revenue and help offset the operational costs.

All information regarding the labs activities, performance, achievements, and member profiles is published on its website. The website also hosts the databases of designs and designers and allows for interactive communication between parties. In addition to the website, the lab uses social media (such as Facebook) as well as e-Newsletters and mainstream media channels to disseminate information. The lab occasionally hosts open events, networking seminars and publishes articles to build up a following and partnership with business. The have participated in promotion campaigns, trade fairs, expos, conferences, and workshops. Traditional means have also been used to promote the lab though the use of brochures, flyers and ad campaigns. 
To conduct its operations, the lab has established two buildings as its main infrastructure: A Technology Incubator and A Technology Center. The Technology Incubator is a building that offers modern office space, laboratories, conference facilities as well as training and business services. The incubator helps accelerate and support companies at every stage of innovation development (from idea to market). The technology center is an initiative to attract foreign and domestic investment where companies may establish their base of development. These two act as production Halls that enable entrepreneurs and investors to develop innovations and promote the region as a growing business sector. Members who participate in the lab receive professional services that help with the development and deployment of their innovative products and services. The lab can also help in finding investment and or expanding the market of a company to forging shores. KTPLab utilizes three different groups as users to help participate in the labs activities. First, the SME's and national/foreign investors who have identified needs take part in the creation of innovative products from the feedback of end-users. Citizens and the local community are involved in the research phase providing feedback on usability and functionality of the product commenting on the process of use. This group eventually becomes the consumers of the new products. The third group is local authorities. The authorities become involved during the workshops expressing the needs and making executive decisions on what projects to undertake. All parties can participate and interact together through open meetings, petitions and inquires.

\section{A.18 Knowledge Micro, Small \& Medium Enterprise Living Lab (KMSME LL)}


KMSME LL is a SME whose objective is to establish a Knowledge Society Ecosystem (KSEco) that is conformed by a plurality of Micro SMEs (MSMEs) knowledge networks (KSNets) that spans over Mexico, Europe and Latin America. It is a lab focused on the economy of knowledge based on a new generation cloud computing services with facets of smart cities; it creates a combination of contextual knowledge networking. This type of platform allows for the exchange of knowledge from various domains and allows the following of global shift trends. With the new expansion of cloud services and social media, the economic barrier is shifting towards digital literacy. The lab wishes to equip entrepreneurs and MSMEs with smart tools that will empower them to foster creativity and grow their productivity and competitiveness. The lab intends to positively influence productivity, employment, production chain and economic growth. KMSME LL basically acts as a platform for MSMEs to collect knowledge for specific purposes. It is especially more powerful when connected to social media as it generates productivity, governance, and privacy. The advanced query and inference capabilities provide analytical feedback to help measure innovation impacts. The platform provides a methodology to for innovative co-creation.

Its owners and board of managers govern the lab. Alfredo Sanchez chairs the decision making process with the help of the Governance advisory council and the Scientific advisory council. The structure is broken-down in Figure 20. 


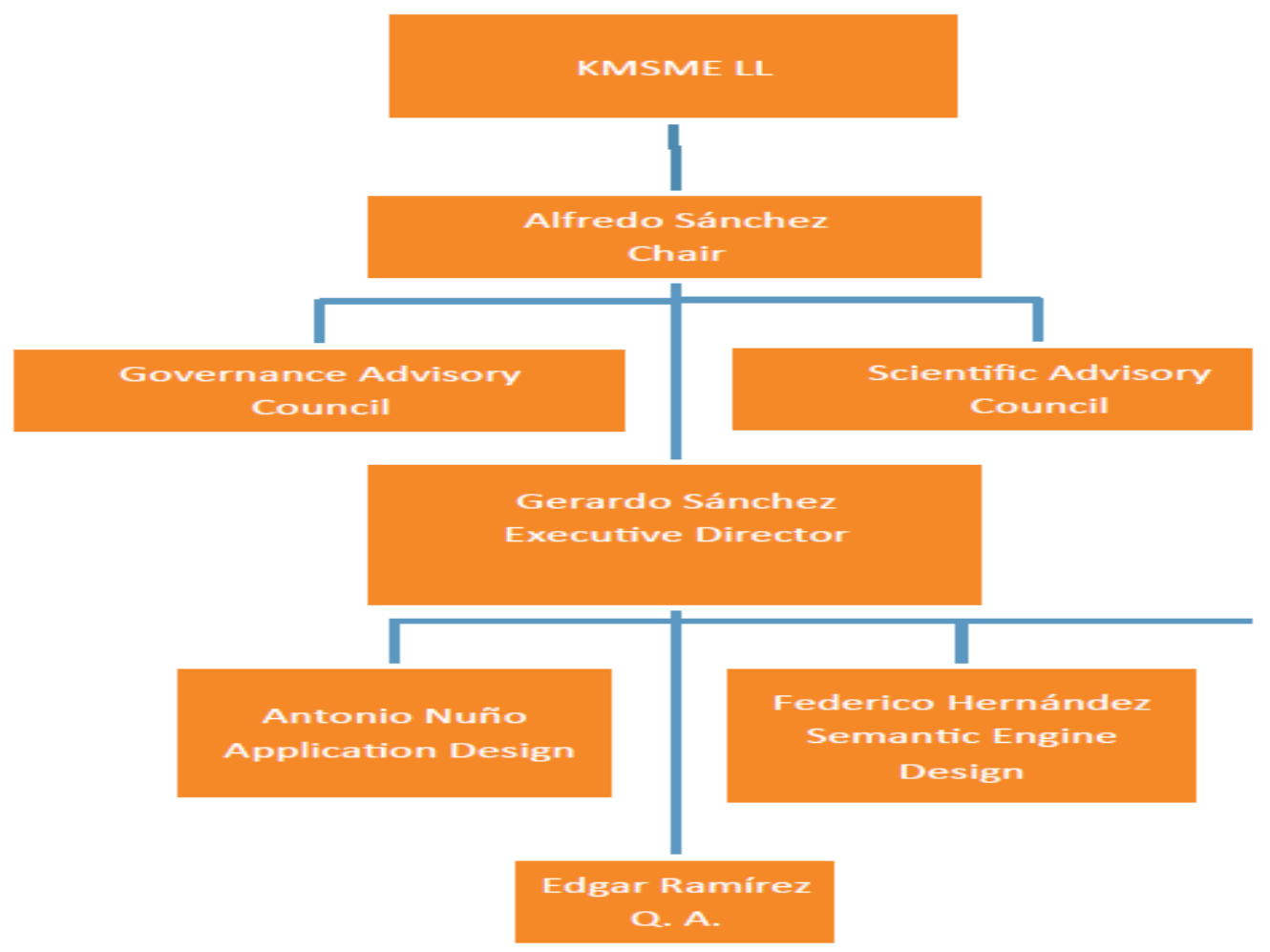

Figure 20 Governance structure of KMSME LL (ENoLL, 2014)

KMSME LL supports several activities as part of its mission. It is building an ecosystem of advanced knowledge tools and international partners to establish its membership with ENoLL. The lab contributes to the global knowledge using its metrics to the development of the region and knowledge exchange. They identify patterns in social behavior, market trends and interpret the data collected for the purpose of the projects.

The lab is committed to openness and collaboration. All innovation developed are patented in Mexico and United states to protect the members rights to the labs output. Members of the lab receive consultation expertise the include design, configuration, ontology expansion, prototyping, community management and digital marketing. The 
members also get access to the advanced tools that connect several organizations together enabling communication features that network, promote and interact with specific audiences.

Funding comes primarily from the owners and founders of the living lab. However, the lab also receives financial support from several private investors and public funding from government grants. The World Bank and Interamericain bank have helped support the lab. The lab also conducts consulting services, knowledge network outsourcing on the cloud and digital marketing for the purposes of creating a sustainable revenue source. A main pillar that the lab has founded its financial strategy on is the sale of its digital marketing services.

Users only require an Internet connect device with a built in browser to participate in the lab. The required technology that powers the lab is the Spribo technology platform that is based on a semantic engine and knowledge model, semantic social web application, interfaces, multimedia content management, web portal container and cloud systems. The lab harnesses the open development of social networks and the best practices of coding to develop software that integrates with the necessary data sources such as Facebook, twitter and Google. The lab also requires the Web 3.0 standards for cloud computing with agnostic capabilities to process knowledge models and deliver tools for online communities. The software for managing the data needs the ability to draw social graphs a do semantic analysis, content analysis, knowledge commerce and marketing. 


\section{A.19 Knowledge Women Innovation Space Living Lab (KWINS LL)}

Knowledge Women Innovation Space living lab (KWINS LL) is based out of Mexico City, Mexico with the aim of supporting personal and professional women's growth through knowledge and innovation. KWINS mission is to foster women's growth, provide motivation and set an open environment for entrepreneurship so that they may achieve their goals, realize their role in society and contribute to the territories economy. The lab currently targets Mexican women and convenes public and private institutions to help with finances, education and mentoring of the women in topics of health, personal growth, taxes, legalities and exports. Municipal authorities also help support the lab by promoting events and providing research work from universities and research centers for the women. The lab has become an ecosystem and social network for the Mexican women where they can access and contribute tacit knowledge and support. The lab wishes to further expand its social network into areas of digital literacy, prevention of disease, space development and employment generation. KWINS LL currently is accumulating a network of universities, living labs and experts to form a creative and competent group of women who focus on social impact. They conduct educational seminars on how to become financially independent, growing their self-esteem and sharing success stories. The lab also offers workshops and webinars and other forms of interactive online education for free to develop job skills, digital literacy and entrepreneurship. The lab also provides a network of professionals who offer legal advice to women with certain profiles, free of charge. The lab also publishes books about 
violence and inequality targeting young generations to prevent future gender schisms.

KWINS offers to women laboratory tests at no cost.

The lab has set up a governance structure headed by Pronea Labs to control and operate KWINS LL activities. This structure is seen in Figure 21. Operational decisions are made by the board of managers while strategic decisions are established quarterly by the Scientific and governance Advisory Councils.

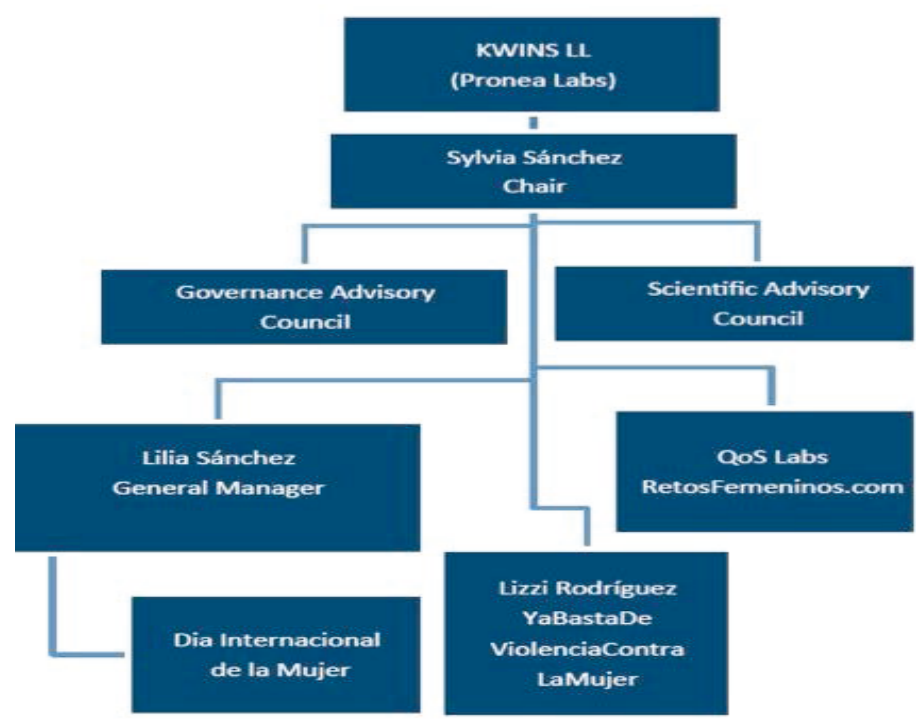

Figure 21 Governance Structure of KWINS LL (ENoLL, 2014)

As a social network built for the support, growth and education of Mexican women, KWINS LL requires a substantial level of communication to maintain its activities. As such, they have strategically employed the use of popular social media sites (Facebook, 
YouTube and twitter) to launch a viral marketing campaign. They have also used radio, magazines and TV interviews with the assistance of celebrities to help promote their events. The events are a form of face-to-face communication held under a more controlled program hosting seminars, workshops and informational sessions for the attendees. KWINS LL also uses their own hosted website, whom they have conFigured themselves, to strengthen the conduit with its user group. The website has management tools to help manage photo and video sharing, blogging, and publishing other content to interact with the members.

Large Mexican-based companies such as Telcel, Telmex and Aeromexico help sponsor the lab financially. International companies such as Coca Cola, Kimberly Clark and Goodyear in addition to Nestle and AstraZeneca also help support the labs activities as needed. Government authorities in Mexico City, Guadalajara City, and Nuevo Leon state also support the lab. It is the intent of KWINS LL to become a self-sustaining lab by offering various services to companies to generate revenue. The aim is to offer website maintenance and management services for a fee as well as receive payment for brand sponsorship. The lab is open to members of RetosFemenions and other women seeking help. It also well publish and release non-copyrighted and non-confidential material (unless otherwise restricted by authors) available to the community. All IP and copyrighted material is kept confidential and protected under the living lab rules. 
KWINS LL does not require anything sophisticated in regards to infrastructure. It simply uses open standard Web technologies such as Web 3.0 and cloud computing to operate its social media and knowledge web platform. The lab uses Ning social networking platform and Spribo knowledge networking platform. KWINS LL does not have proprietary technology but rather is owned by Glam Media/Ning and QoS Labs. Women can group together into small companies where they contribute their own visions expressing their creativity without prejudice.

The lab has forty thousand members enrolled in participating in the labs ongoing activities. The lab uses its website and social media to drive the interactions between the community and facilitate the exchange of knowledge, ideas, suggestions and experiences. The lab uses Google analytics to observe the interactions measuring the member's demographics, visits, and average time on site, new visits and bounce rate. Women who participate in the lab get the intangible benefits of knowledge and moral support. However, women can also receive scholarships through the lab to help them invest in their personal growth through education and business ventures, which the lab is more than willing to help support as well.

\section{A.20 LabICT-PA ICT Laboratory for the Public Administration}

The Italian living lab named LabICT is a public organization that is hosted by Lepida SpA; an in-house providing company that was established by Regional Law whose activities are part of the Regional ICT plan. The lab is part of the Emilia-Romagna High 
Technology Network structured as a public and private partnership. The lab's objective is to strengthen the industrial research and technology transfer network, fostering economic growth and competitiveness. It hopes to achieve working prototypes of innovation to showcase the potential of the living lab and allow for measurements of performance. Its primary focus right now is towards developing and testing ICT services for public administrators and to become the regional governing body over ICT. Not only does the lab involves the regional government in these innovations, but includes Universities and enterprises in the innovation process and continuously coordinated collaboration between them. With the new partnerships, the lab wishes to extend its innovation domains into Smart cities, Future Internet, Internet of things, Social innovation, Social Inclusion, Egovernment, E-participation, Security, and Smart Regions.

As a public organization, the lab is governed by the regional authority and has been assigned to Lepida SpA to be stewarded. Lepida SpA is also governed by public law with shareholders in the Regional government and local public administrators. The lab and its activities are coordinated by this organization. A scientific committee as been established by the organization to coordinate the strategies of the lab and evaluate/ensure proper operations of the lab. The regional government also inputs their strategies into the activities of the lab. Five other leaders have been appointed to oversee the implementation of various activities. The lab is financially supported through government grants from the Emilia-Romagna Regional Government. The lab is currently formulating a business model that adopts a co-financed position from deploying the output products of the lab. 
LabICT believes in the openness of innovation and tries to use as much open source software as possible. As such, the lab makes available the all stakeholders the innovative know-how included in their co-designed projects. However, the lab does limit its use to the original scope of the application and gives permission to the business to commercialize it and expand it into other markets.

As part of the initiative to develop ICT services, the lab has undertaken work to establish video surveillance, sensor networks and mobile applications. The lab has already conducted a survey of tree thousand citizens to begin investigating and collecting feedback on the launched e-government system. Likewise, the lab is coordinating its efforts with universities and companies to gain momentum for their activities. The lab uses its website as its primary portal of information dissemination. Newsletters are also utilized targeting a large audience of citizens with heterogeneous backgrounds. LabICT involves over four hundred pubic entities and eighty business partners. The critical mass of the large amount of members accelerates and promotes the collaboration of co-creation and testing of the innovation solutions. The diverse expertise brought by such a large network stimulates cross-pollination and acts as a think tank designing scenarios, services, and products that favor citizens and market.

LabICT harnesses the regions built in networks and infrastructure as its own test bed. The lab grants benefits to its private members ensuring them commitment from the government. Presented with the research framework, the lab also has evaluation metrics 
and benchmark data to help improve product development success. Methodologically, LabICT works with its users to set the out the project requirements and specification. The companies design and build the products and allow the users to test to validate their functionality.

\section{A.21 LeYLab}

Light and You Lab (LeYLab) is a living lab hosted by Alcetel - Lucent Bell in the Country of Belgium. Its mission is to test innovative applications over a NGA infrastructure because fiber offers advanced bandwidth and QoS. Through this mission, the lab aims to provide multimedia services to anyone on Internet connected devices such as tablets, laptops and TV. This lab will stimulate the ICT sector to encourage development for fiber technology and lower the entry barrier for "internet-illiterate" panel members. Its domains of exploration are multimedia and e-health applications. LeYLab expects to advance the multimedia domain through development of application in Smart Cities, Internet of things, e-Government and Creative Media. It also believes that development in e-Helath will build a network for well-being and further the Social innovation and Social Inclusion of participants. The lab does not consider itself as a technology agnostic cloud providing an open-ended test bed nor a technology laboratory research lab; it is an ecosystem of end-users, technology and specialists working together to work on a common goal of boosting ICT innovation. It is a means to optimizing life of care-demanding citizens by extending their possibilities through ICT. 
The living lab help lower barriers to ICT as to stimulate innovation and help pioneering companies to understand the market, accelerate their development and improve the quality of their application. Users benefit by getting access to new and improved applications at lower cost in exchange for their feedback. Additionally users can access the application anytime anywhere. All members get access to professional support and help from expert consultants. The lab promotes its activities heavily to encourage continuous submissions of new applications such that a sustainable business model may be established in the future. The lab actively supervises the projects preventing deterioration in perception of the application and interference between different providers.

The lab has setup a steering committee to monitor and discuss the overall progress of the project and to align all activities with the labs mission. They meet regularly to stay up to date on the labs conditions and to readjust misguided activities.

The living lab upholds and the use of open standards for collaboration emphasizing its advantages in integrating other innovators products. LeYLAB has prepared a consortium agreement that outlines IPR rules and governing philosophies for all members of the living lab. The agreement removes the fear of members attempting to claim ownership of IPR without clear and mutual upfront approval by the lab. This agreement also dictates how additional members are allowed to join. LeYLAB uses its own website to communicate with its participants. However, it also uses direct mailing lists to deliver informational papers and official documentation. They have taped into the social works 
system, and local organizations (schools, theaters, hospitals etc....) to deliver more direct presentations to citizens. Press releases and the cooperation of the minister of justice and administration have all played a role in the promotion of the lab.

The LeYLAB consists of a consortium of over three hundred members composed of large and influential partners. There are twelve owners and investors: Alcatel-Lucent, Androme, Belgacom, Comsof, IBBT, In-Ham, the city of Kortrijk, OCMW Kortrijk, Regionale Mediamaatschappij (WTV-Focus), Televic Healthcare, U-Sentric and Videohouse. The leading telecom providers supply the equipment setting up the necessary infrastructures to provide high-speed Internet. Specialists in multimedia provide storage and distribution with seamless experience that's accessible to member citizens. Doctors and health specialists provide expertise and collaborate to build up an ehealth infrastructure. Research institutes manage the panel of test users.

Funding is expected to be rooted in a business model that sources revenue from integration and support activities provided to users. This model is intended to cover operational costs and over time pay for the costs of the equipment and its setup.

The set up infrastructure is meant to bring Internet connectivity to the citizen's homes at unprecedented speeds removing the limiting factors of introducing new applications. This infrastructure is a networked series of Fiber technology setup with industry standards to optimal device management, life cycle management and sharing of multimedia. Open source APIs are used for seamless integration of third-party applications within the open source platform. LeYLAB conducts is research through a set of platforms to manage 
data collection and user involvement and behavior for example, e-Health uses video communication to persons with mobility restrictions. The lab allows unrestricted access to applications so that users may test and validate the technology in the comfort of their own home or on the go. After the usage of the applications, the panel users are asked to provide their feedback using online forms or in-person interviews. The lab encourages inperson interviews as it creates social proofing and sense of community among the members. To encourage contributions, the lab offers rewards varying from free services to gift packages.

\section{A.22 LIVING LAB CLUSTER TIC BOGOTÁ - COLOMBIA}

Living lab cluster TIC Bogota is a Colombian lab that consists of several IT, digital, animations and video games enterprises, which have come together to benefit SMEs, as well as national and international industry. They're primary goal is to improve the territories development in smart regions, increasing social innovations by closing the digital gap and improving social inclusion. The lab intends to conduct training, certification, coaching, promotion and audits for digital business and software enterprises. The lab also intends to improve SME decision-making process in every environment, productive and strategic area, with IT network and innovative projects so that they can improve their processed information and offer superior products and services. The idea is to have a more resourceful and territory and therefore the lab will integrate the education system, industry, ICT and government to achieve this. 
Below (Figure 22) is a model defining the structure of the living labs governance. The I+ $\mathrm{D}+\mathrm{I}$ is coordinated by ESICENTER SINERTIC ANSINO which is composed of at least one hundred and fifty national and international organizations. The strategic level of the lab is governed by the Technological plan, which is developed by the Watch Exercises where new technological trends are identified. These are retrieved from interviews conducted with businessmen from industry and general economic areas. All the strategies collected are composed into a roadmap that will outline the work for the next five years of the market conditions and product outputs.

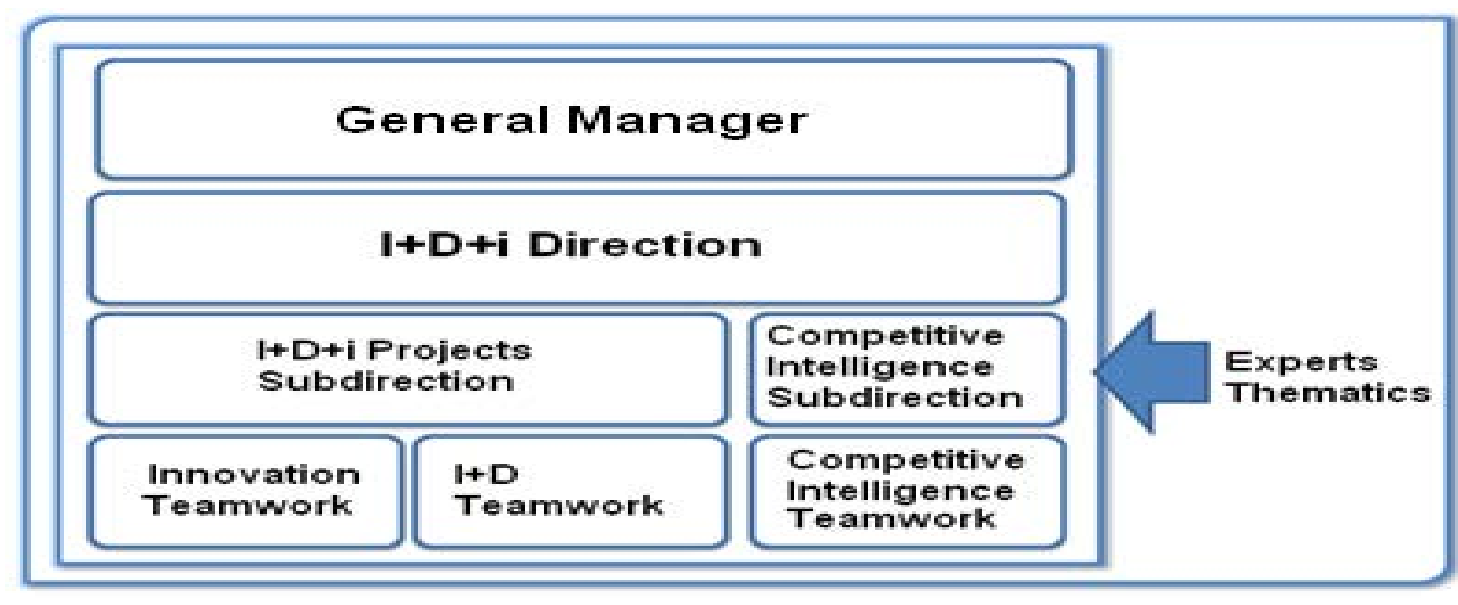

Figure 22 Governance Structure of Living Lab Cluster Tic (ENoLL, 2014)

The lab is currently involved in atomization operations and the development of interactive content for the national education and industry. Members of the lab have already begun outputting video games and digital animation and using the advanced multimedia-testing lab. The lab offers services in client communication, knowledge management, organizational IT, Trend Watch, marketing and business intelligence, human talent management and innovation management. The lab makes available to its 
members services in knowledge management, technology transfer operations and coaching. Members can also expect to gain advantages from the cluster of participants having access to government entities, industry partners and resources from educational institutions.

The lab uses ESICENTER SINERTIC ANDINO web portal for multidirectional communication as way to stay in touch with its members. Currently the web portal is only used to publish news content updating members on the labs activities, however, the web portal is being upgraded to include visual and dynamic animation as well as an access portal. This will enable a true two-way communication channel as well as provide management tools to the members to track their on going projects.

The Living lab cluster believes in openness and that it must adopt a cooperative attitude in order to be innovative. It is through shared knowledge and debate of ideas from different perspectives that innovation is achieved. The lab believes that all members must respect each other and work as a team as an interdisciplinary group. The lab follows Colombian IPR laws when regulating the labs handling of shared IP. There are two approaches to the labs IPR. The first approach is "Moral Rights", which, depending on the projects context and associated regulation would be the property of the producer. Secondly, the living lab would control the IP and license the technology to a company for its commercialization, hence receiving royalties from the profits of the innovation. 
The lab involves as many different organizations to make its activities possible. It has a network of SMEs, big companies, IT societies, government, financial institutions, academia and citizens.

Living lab cluster uses its member's resources as its main infrastructure. Office systems, search engines and development structures/tools are all important gears in the labs groundwork. It also accesses patents bases and scientific bass from its members as an asset for innovation and powered by the communication tools used to network all the participants. The lab is set up with a private business model to generate revenue to help pay for its operational costs and activities. One stream of revenue comes from the royalties generated from the innovation outputs of the lab; the second stream comes from the private services the lab offers. These services include quality inspection, software ratings, innovation management, training and coaching activities to the industry and individual person and ICT transfer.

The lab uses focus groups with experts and professionals to learn about technology trends and market demands. The labs than initiates development projects to produce the projected innovation. With the help of fellow members, the lab can set a benchmark to which the product can be tested against and validated. It can then help transfer the technology (i.e. file for protection and licensing) and help business to commercialize it through its market research. In Figure 23 it can be seen that the lab has broken up the phases of project development into three categories and four steps: Competitive 
Intelligence (idea generation and concept development), Project management (project development) and Protection and Commercialization (Result Output).

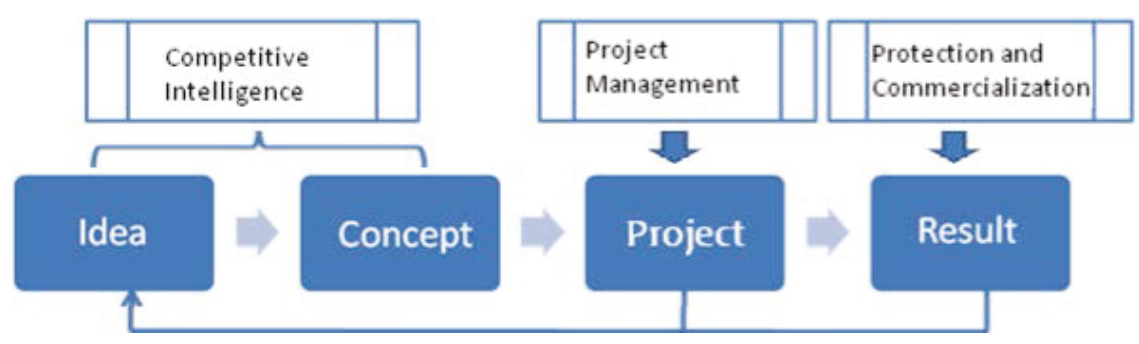

Figure 23 Management during Innovation Cycle of Living Lab Cluster (ENoLL, 2014)

\section{A.23 LL Campiña de Jerez}

Living Lab Campina de Jerez is a Spanish living lab that is structured in a public-private partnership aimed at promoting the regions innovation capacity. The objective of improving quality of life, especially for people at risk of social exclusion, is at the core of the initiatives the lab undertakes in sponsoring an information society, co-creation, technology validation and development of new business models. The lab hopes that these efforts will develop the society to grow the economy of the region. The lab engages in activities to help improve peoples life quality in rural areas and those with disease. The lab thus far has worked in geolocation systems that allowed the tracking of Alzheimer's disease and other mental disabilities through an Internet phase thus improving mobility tools for caregivers and families. Likewise, they have engaged in renewable energy, 
equipping rural areas with sustainable and renewable energy. The lab is working to effectively manage the public resources and infrastructures moving towards smart cities. To spread the word of its activities, the lab attends events and conferences in addition to the array of media channels they use and the publications they issue.

The lab currently is depended on public funds and private company investments. It seeks to create a sustainable revenue model through the innovation outputs to recover its operational and investment costs. This model will maintain a competitive price model that is adapted to the user base and market prices.

A reference person that is highly motived in this methodology represents all stakeholders in the governance model. A monitoring committee composed of representatives from the members, makes the strategic decision regarding new members and projects. They're responsible for balancing the needs of people with market trends to guide the labs productivity. It is the committee's responsible to establish strategic plans and midterm objectives to achieve the labs goals. The lab uses a Steering Committee to manage the labs activities. The committee is composed of four entities, The Local Action Group Campiña de Jerez, The Delegación del Medio Rural of Ayuntamiento de Jerez (Rural Areas department) REQ (Technology and innovation company) and University de Cadiz. University de Cadiz offers management support to the scientific, technical and artistic services to business that come from research providers. They also help with the establishment and management of $R \& D$ projects as well as council on the creation of companies based on the results of the lab. Each undertaken project is appointed a leader 
to oversee it. The lab begins a process of consultation and participatory tables drawing drafts and pilot works.

There are four major stakeholders who commit resources to the lab and aid it its operations. One, is GDR Campina de Jerez, which is a non-profit organization with w multidisciplinary staff and managerial work of a Top-down methodology. Second, Town hall of Jerez de la Frontera, which is the public administration of the region which is needed to help support, license and govern the lab. The government helps connect the citizens and business to the lab acting occasionally as an intermediate between the two. GRUPOREQ is a company that has expert employees working in its R\&D division. The company works with the lab to lead innovations and product development, invest much of their own money into the lab. Lastly, University of Cadiz is an integral part of the labs research initiative.

Infrastructure that has been set up to power the lab's initiative consists of the resources made available by the constituents of the consortium. Buildings with the capability of innovation have rooms equipped with internet and made available for training. Workshop laboratories and program facilities exist in addition to office space for employees. Telecenters are made available for public access for rural citizens. Technologically, the lab has a portfolio of useful equipment to achieve their various goals include: 
For the distribution to final customers and tools for projects, internet- web based geolocalization Remote Control of public spaces lighting

- RF Electronic Systems platforms and web services Servers

- Ip Phones

- Remote Control of a Home Automation

- Ip Security

- Call center VoIP.

- Routers

- Wi-Fi

- Minipc

- Antennas

- Localizers

- relays, for Home Automation

- circuit interruption

For requirements of end customers and the need to give more coverage where cable does not reach and better serve separating the voice and data

- Cameras

- Attendance Control

- Biometrics

- WiMAX coverage

- 100 Internet access points by $5 \mathrm{GHz}$ radio frequency 
For Control and energy savings:

- Remote control lamp management

- Solar panels

- Wind Turbines

- LED Luminaire

- communications towers

- Optics Fiber

- Renewable energy distributors through solar panels and wind

- Biometric Attendance Control

The lab uses a selective opening model when it comes to allocation, marketing, modifying or distributing the product. The lab acknowledges that it must unlearn old paradigms of closed and internal thinking and begin to adopt a more open and communal philosophy. The lab involves a bottom-up methodology putting users at the center of the innovation process. Their role is important for the idea generation and testing of the prototypes. Users articulate their needs and define the specifications for the technologies to be built. They then assess the products and provide their feedback generating knowledge on the subject. The technical staff is always in contact with the users supporting them in anyway in order to maintain an deep understanding of the experience and develop a tailored solution. This communication and work can occurs through various forms of channels including direct dialogue (interview), social participation 
(workshops, forums) or environmental experimentation. Internet powered tools are provided to populations scattered in remote a rural areas to engage the citizens.

\section{A.24 LUSAGE}

LUSAGE is a living lab located in Paris, France that specializes in the design, development and assessment of technology-based products and services for the elderly. The lab is owned and operated by the Assistance-Publique Hopitaux de Paris (a hospital group) and the University Paris Descarts. It is located in the center of Paris where health professionals and elderly can access with ease thanks to public transportation. LUSAGE aims to help the elderly optimize their cognition and autonomy by offering technology solutions that will help manage their daily-life activities. The technology solutions will also help caregivers cope with the patient's needs and situation. They refer the domain of designing, developing and evaluating assistive technology products and services for the elderly as Gerontechnology. As part of the academic research team, LUSAGE focuses on research approaches through collaboration with other laboratories in different fields such as neuroscience, sociology and philosophy. They intend to identify and share best practices among the labs and offer innovation in the field of health care to the hospital centers.

LUSAGE has a steering committee that is composed of LUSAGE team members. The team meets once a week to discuss the organizations strategy and review the on-going work. The steering committee is important for reviewing the organizations progress, 
evaluating potential risks and suggesting strategies to anticipate and minimize these risks whenever possible. The team also hosts monthly seminars to update the group on scientific works of the members. Together, the team is responsible for managing the process of the lab and is in charge of the research planning. As an open platform the team must ensure the lab is transparent and inclusive with regard to the stakeholders making all scientific publication accessible. An appointed coordinator with support from a scientific team helps decide on research objectives and methods. The financial and administrative board is responsible for administration and legal issues and the financial management for the lab.

There are many stakeholders involved in the LUSAGE that can be divided into four groups: users, partners, industry partners and networks. The user stakeholders include the health professionals such as doctors, psychologists, speech therapist, occupational therapist, physiotherapist, and social workers. The elderly, both healthy and those suffering from cognitive impairment are also part of the user group. There are many partners ranging from associations to academic partners and industrial partners. The following is a list of organizations who are partner stakeholders: E-senior, old-up, Centre Local d'Information et de Coordination Paris13-14, UMR 894, Ecole des Mines de Paris, ISIR (U. Paris 6), VALORIA (U. Bretagne Sud), Laboratoire TIMC-IMAG-UMR, CCIP Groupe ESIEE, Université d'Evry Val d'Essonne, IBISC, GET/Inst. National des Télécom, University of Reading (UK), Technical University of Ilmenau (Allemagne), Austrian Research Centers GmbH -ARC (Autriche), University of A Coruna (Espagne), ESIGETEL, ROBOSOFT (Bidart), LEGRAND (France), CGx-Systèmes SAS, AKG 
(Autriche), METRALABS GmbH Neue Technologien und Systeme (Allemagne), SmartHomes (Pays-Bas), CURE (Autriche), SABIA (Espagne), Lab-in-Ham (Belgique), INGEMA (Espagne), Fundacion Robotiker (Espagne). These partners provide medical support for the management of the elderly and help promote collaboration with other laboratories. In addition the partners, LUSAGE also invted members from network organization that help expand the reach of the labs support and influence, these include: Réseau de consultation mémoire d'Ile-de- France, Fédération des réseaux gériatriques d'Ile de France, Fédération des Centres Mémoire de Ressources et de Recherche français, EADC, INTERDEM, ISG.

The management of IPR in the lab is done so through the consortium agreement that is signed by all participating members. The lab includes a non-disclosure agreement in the consortium agreement to control the sharing of information during the set-up of the project, patent licenses, software protocols and more. All IPR issues are managed in conjunction with the Office of Technology Transfer and Industrial Ventures.

The Agence Nationale de la Rechreche funds by the European Commission and LUSAGE. Other organizations such as the National Fund for Solidarity and Autonomy Caisse Nationale de Solidarité pour l'Autonomie, the Region Ile-de-France, the Regional and National Programs for Clinic Research at French Hospitals, the French National Plan for Alzheimer's Disease, the France Alzheimer's Association, and the Fundation Médéric Alzheimer also help finance the living lab. The lab also receives revenues from private contracts constituting services rendered in the field of usability, accessibility, and 
adoption of technology by users. They also conduct training for health and offer social and/or technical support for dementia and healthcare.

LUSAGE has a pool of resources comprising their living lab infrastructure. Hospital facilities and configurable home environments are used for real-life healthcare test beds. The academic facilities, used for research labs, and the rooms available from the university and hospital function as meeting places for focus groups and conferences. From a technical stance, the lab uses software for behavior analysis, eye tracking applications and statistical programs to analyze the data. As part of their lab capabilities, LUSAGE uses laptops, tablets, smart phones and video game consoles, robotic technology, camcorders, eye tracking technology, sensors, and other audio recording systems. They have access to MRI machines and other medical technology as the need arises.

LUSAGE as its own website to post updates about projects, news and publications. The most prominent communication channel they use is through blogs, newspapers, journals, radio and TV channels that invite the lab to talk about their activities and mission. The staff members independently promote the lab through their publications and lectures they give about the research they conduct in the lab.

The lab engages its end-users throughout all stages of the innovation process. The participating users are fully informed about the projects and are asked to give written consent of their involvement. The lab upholds all ethical requirements during its activities 
and allows the users to withdraw at any time. All research is conducted with a close link to all stakeholders, maintaining all necessary support services and management of the elderly. The users help identify the needs and also test the technology on behalf of the developers. The lab conducts the analysis through follow-up studies and assesses the motivational reasons for using the technology and the level of adoption observed. It is the developers (engineers) who actually design and develop the technology by incorporating the users feedback. Professionals help by providing evaluations and scientific knowledge and sociologists provide analysis of the use of technology after observing the users and collecting their feedback. Academics cover an array of activities including evaluations, analysis and most importantly, the development and implementation of technology for the purposes of research. There are many advantages to participating with the living lab. As an end-user, you can expect to receive tailored innovations that address particular needs and preferences aiding in the day-to-day activities. Health professionals also receive experience on new and upcoming technologies that will shape the future of caregiving to the elderly. Lastly, academics have the opportunity to use the labs resources, particularly the test environments, to conduct proper research while maintain an open channel of communication with the end-user in mind.

\section{A.25 Málaga Living Lab}

The public agency known as Malaga living lab found in Spain is an ecosystem of living labs (in the city) and their associated innovation projects. Built as a public-private-people partnership and promoted/supported by the City Council of Malaga, the lab hopes to 
harmonize together the living labs of the city and create a synergy to define a common methodological framework for user involvement. As an ecosystem of various living labs, Malaga living lab becomes a multi-project and multi-domain oriented with focus in energy, smart cities, sustainable mobility, eparticipation, social inclusion, tourism and social innovation. As of 2009, when the city of Malaga became a smart city with special focus on energy efficiency, it became the labs objective to converge the ecosystem and bring together the local actors to promote the continued development of Smart City technology. It encourages the adoption of an inclusive habituate to enable co-creation where everyone can develop his or her projects without limitations. The lab will provide tools, offer public services adapted to citizens demands and grow entrepreneurship. The lab uses its own website and those of its members to communicate with its members about the projects. The lab also uses social media and traditional channels such as newspapers and radio to also stay connected with citizens.

The lab has structured a thin governance structure to maintain openness and flexibility. As a public-private-people partnership, the lab has modeled its governance on the City Councils structure in coordination with the rest of the core entities. Malaga living lab uses a Steering Committee, composed of representatives of core members, as a management and not an operational arrangement. The Steering Committee only adds value to projects by providing guidance and support to user involvement, methodologies and merging projects. Each project has its own structure and governance and operational model. This setup provides coherence to the decision making process without losing flexibility in the creation and adoption of new initiatives. The lab does not believe in 
ruling over IPR issues with a generic agreement but rather deals with IP on a project by project defined by their respective consortium of participating members.

The lab has categorized its stakeholders into three different groupings: core members, project participants and living lab followers. Core members are organizations the help promote and support the living lab. They define and implement the labs strategy, make decisions for projects and initiate them as well as interview and approve new members. The core members consist of:

- Malaga City Council (Different departments: Civic participation, Immigration, Development and Cooperation Department, New Technologies Department and Observatory of Urban Environment)

- Municipal Energy Agency

- $\quad$ I2BC (Research Innovation Center)

- University of Malaga

- KIC Malaga (Knowledge and Innovation Community)

- Endesa (Electrical Company)

Project participants consist of public and private organizations or associations and research groups that get involved in the labs projects and help add value to the research. Living lab followers are those that closely follow the activities carried out in the living lab and sometimes get involved in the simple activities of the labs operations. The 
followers consist of local SMEs, citizens, civic associations, NGO's and other institutions.

The lab has independently structured a business model and allocated financial resources to each project. These projects are funded through private and public finds. The lab does not have a formal structure that offers funds to specific projects.

Malaga living lab's infrastructure is supplied by its core members and consists of physical assets but also human resources, knowledge and networking resources. The members offer up their premises for the lab to host meetings, workshops and other cocreation purposes. Below is a list of physical assets that the members have provided to the lab for the purpose of innovation and co-creation:

- Municipal Wi-Fi network:

- Multi-service fiber network:

- Dynamic Digital Signage Network:

- Virtualization system for urban traffic control cameras:

- Municipal Wireless broadband network:

- Municipal energy tele-control system:

- Geographic Information System (GIS)

- Electric municipal networks and the public lighting network. 
The lab uses the citizens as one of its main pillars. All living lab projects are carried out to deliver value to the city and its citizens. The citizens through their association or local SME initiate some projects. Citizens can help by defining the requirements of the projects or testing the products and services. All projects conducted by the lab are performed in real-life settings such as their homes, on the street, city website, or through the broadband networks. Private corporations carry out the implementation of the designs and activities of the products or services while research institutions conduct the research within the real-life scenario. The exact methodologies used to conduct the living labs research is being derived from the harmonization of the other living labs in the city. The synergy between the labs and public agencies can be used as a tool to improve the efficiency of the research in identifying and acting on the needs of the citizens. The labs research communications are transcribed on official documents, in magazines, newspapers and media channels such as radio TV and websites. Surveys, focus groups, evaluations and meetings are held through interactive websites. The recruitment of citizens for testing purposes is done through civic associations in order to maintain legal and ethical rules. The association informs the citizens about the goals of the project, receive signed consent and protect the citizen's information.

\section{A.26 Man \& Earth Living Lab}

The Madrid living lab, Man \& Earth, is an initiative designed to insure a balance is maintained between human activity and nature preservation. Man \& Earth works for the 
regional and rural development of Spanish territories through the implementation of various activities that have been conceived by the needs of the people and the environment. The lab's vision is to apply open innovation methods for rural development while maintaining its mission and focus on sustainability, biodiversity and cultural heritage. All related fields such as social inclusion, sustainable energy, climate change, health and wellbeing that can improve the balance between peoples needs and those of the environment will be explored. The Man \& Earth living lab will conduct R\&D research to enhance the quality and interests of its activities. The lab has partnered with rural populations, producers, public administrators and technology providers.

The Executive body and Advisory board make all strategic decisions for the lab. The Advisory board, which is composed of representatives of the members, holds binding decision power as governed by the bylaws. The various projects that the lab undertakes are coordinated by their project leaders who are appointed from the coordination organization, which is comprised of the five pillars representatives.

Man \& Earth living lab offers geolocation of rural products and services. This current project is aims to track every asset in rural and natural territories to be able to later increase the value of these territories. The lab also sells its "Quality Mark" brand in collaboration with Felix Rodriquez de la Fuente Foundation to those actions, projects and or products that have agreed to the objectives and quality standards of the lab. Man \& Earth lab also participates in conferences, seminars and training days to educate and raise awareness about biodiversity, sustainability, rural development and rural/urban connivance. Lastly, the lab is researching new techniques and business management 
models for the purposes of rural development and biodiversity. These new models and techniques are aimed at preserving the wild biodiversity and cultural heritage of the areas.

The lab prepares and signs all participants unto the Collaboration agreement that outlines the engagements of the members and defines their operations within the Man \& Earth framework. Man \& Earth respects IPR laws and regulations. In the event the lab obtains results or is involved in other projects that have been initiated prior to their involvement, it adopts the rules originally agreed upon of that project. However, projects that are developed my the lab or are funded (even in part) by public investment, the IPR becomes open so that it may be exploited. The idea of such philosophy is to enable openness and allows society to benefit from the outcomes. When the projects are funded my private investment, the IP is protected and then commercially developed to return profits on the investment.

There are five main stakeholders in the Man \& Earth LL: Rural Professional Organizations, Local Action Groups, Non Government Organizations, Enterprises and Public Administrators. These members have agreed to and signed the Collaboration agreement to officially join the living labs consortium. In addition to the five pillars, there can also be stakeholders in the form of participants and or investors. The mention and other organizations are free to get involved in the actions of the projects and instigate new projects as long as they follow the strategic framework of the lab. Likewise, investors can choose to fund projects given that they agree to the objectives and governing of the living labs framework. 
Man \& Earth receives funding from private and public sources depending on the projects. Private investment sponsors projects that test and validate products or services via users in the territory where the company expects to deploy it. The lab has formed a self sustaining financial model where by they sells products and services in the fields of sustainability and biodiversity for the rural development and land stewardship. This model help offset the value of the operational costs of the labs activities.

Currently the lab is working to consolidate its entire infrastructure into a single and more manageable organization. Currently, each project has set up its own website, but the lab will harmonize the them into a single portal that is capable of organizing, showcasing and communicating all activities, products and services of the lab. The lab uses blogs, forums and other interactive tools supplied by the latest web standards to facilitate communication between members and to conduct is needs crowdsourcing. Face-to-face or digital meeting are the primary ways of training and harnessing idea collaboration. All validation of the developed product takes place in realistic environments to proper testing. Man \& Earth also has, at its disposal, an augmented reality that presents the forestation developments on users mobile phones. The 3D technology can also be used for educational purposes especially children. Lastly, the lab uses geolocation tools that can sense and locate itself in relation to a map.

The primary benefit that members receive from participating with the Man \& Earth living lab is learning and using the new methodology/techniques in developing sustainable 
business models for meeting the needs and humans and environment alike. Entrepreneurs can use such a method to find a niche market in the green market.

\section{A.27 Middle East Digital Ecosystem Living Lab}

Saudi Arabia's first living lab is called the Middle East Ecosystem or MEDELL for short. The lab aims to build a cooperative network of government, academic and research institutions for the purpose of developing the countries digital ecosystem. Specifically, MEDELL wishes to develop solutions in the field of eGoverment, eHealth, eSecurity, eEnergy, eLogistics and eBusiness. The ecosystem will help facilitate the knowledge transfer between parties through a developed interactive training framework and integration of business processes and models. As such, the labs mission is broken into two initiatives: 1) To jointly manage the technology incubation process and 2) jointly manage business incubation process. Its first initiative involves close interaction with researchers, policy makes and business communities in order to enhance the innovation process. This initiative is intended to output real solutions and innovations for the purpose of commercialization, for which, its measured metric is the level of investment it attracts. Their second initiative is aimed at building a deeper interaction with technology entrepreneurs and venture investors to build viable and innovative business. The lab hopes to produce spinouts with competitive business plans, functioning management and innovative products from this initiative. 
MEDELL is owned and operated by SISCOM. As owners, they provide the strategic direction, leadership and financing to operate the living lab as well as networks to attract further sponsors, investors and partners. SISCOM has structured for MEDELL two levels of governance to federate the activities and projects of the lab. The Management Board, primarily SISCOM, consists of the senior representatives of the entire core team members. This level democratically develops an approach for the living lab and sanctions the implementation of projects. The Steering Committee is composed of the general project coordinator, project manager, technical coordinator and work program leaders. The lab hopes that the European commission project officers can participate in these meetings. Meeting twice a year, the Steering Committee is responsible for reviewing and setting technical and economic aspects of the project and develops the future direction of the lab. The steering committee is to pass decisions using a consensus model, however, when disagreement occurs, an organized vote as outlined by the consortium agreement will prevail.

To date, SISCOM has been a key operational in ICT-based system solutions provider. The lab has created nodes of innovation for business, facilitated collaboration with research institutions and academia, and has worked with government authorities to develop ICT application and services in eBusiness, eGoverment, Energy, Security, eHealth and eLogistics. MEDELL has also launched a Women eBusiness Incubation Center to empower women and encourage entrepreneurship in Pakistan.

MEDELL utilizes media channels such as TV and radio to raise awareness of its endeavors. It has also created its own public website and engaged in social media to have 
an online presence and connect with its members. Most importantly, the lab has created its own brand and logo to enhance its public visibility during its communication strategies.

MEDELL fosters a philosophy of openness and enforce IPR principals on a project-byproject base depending on their nature. The lab respects IPR in accordance with the agreements signed and laws and industry standards. MEDELL holds to the belief that innovative effectiveness from their collaboration is achieved through bottom up involvement and the cross-pollination of knowledge from private, public sectors and enduser alike. The lab therefore involves local and national government organizations, academia, science parks, non-profit organizations and SMEs. These stakeholders assume certain roles as part of their participation in the living lab framework. Researchers from academia produce creative technologies. Business communities bring market knowledge and experience that help shape the application of the new innovations for the customers. Policy makers mediate between researchers and business communities to help development and provide national priorities to their projects. Involved technology entrepreneurs help produce innovative technology in response to market demand while the business entrepreneurs help shape the member companies into more competitive and investible firms. The venture investors help support the entrepreneurs and the companies.

MEDELL has not specified any specific resources or infrastructure it requires but instead notes that a generic ecosystem infrastructure with common support, services, components, generic integrated solutions and infrastructure is all it needs. 
As a digital ecosystem, MEDELL provides components, modular plug-ins and knowledge sharing facilities to its members in addition to the networking opportunity it offers. Such an ecosystem can help raise investments; showcase technology and crossfertilize company projects for innovative outcomes. MEDELL offers a unique opportunity to enable the creation of business clusters with tactical and strategic alliances to act collectively in researching and scaling innovation. Businesses can modify their process based on feedback from user communities thus reducing risks in product investment and market launch. MEDELL also help decrease time to market by improving the quality of information and knowledge exchange which impacts the efficiency of production and addressing to customer needs. Users can count on receiving reliable innovative products in return for their participation. Their continued participation will ensure the continued investment and project development of new products from the living lab.

The lab uses an intranet network to share and collect information about the projects it is researching. The lab has a two-phase operation to complete the cycle of design to deployment into market. The first phase is a collaborative operation between academia, business and policy makers to help set the requirements and develop the necessary technology. Once completed, the technology is handed to the entrepreneurs where they validate the product, map out a business plan and then commercialize the product. 


\section{A.28 MIMMALAB - Interactive Music Museum Living Lab}

MIMMALAB is an interactive music museum living lab that has been in operations since 2002 in Spain. The museum was created and is supported by the City Council. It is currently being operated by a local SME called InterExpo, which has recently taken on another SME called Emotional Exhibitions S.L (E2). This SME offers technology and consulting services to the museum to transition them from a traditional operation to a more interactive and user centered business. The lab takes on the form of a public private-people partnership between the SMEs, government and citizens. Unicaja, Malaga City Coucnil, Diario Sur newspaper and McDonald's sponsor it. The aim of this living lab is to use Open innovation methods as a tool to bring technology and experiences closer to people via the resources of SMEs, research institutions, music institutions and associations. Currently the lab believes in an open model and is willing to accept to members into the living lab ecosystem. Concrete rules and regulations that regulate the dynamics of the living labs philosophy especially in IPR have not been established. Instead, the lab deals with IPR issues on project-by-project bases.

MIMMALL stakeholder network is a limited one consisting of the founding SMEs InterExpo and E2, and the City Council and twenty-five hundred citizen "friends". The lab operates using a combination of public and private funding as well as revenue from the museums exhibitions, space rental and services rendered to third parties. The objective of the lab is to move from loans and public funds, to a more private and sustainable financial strategy through membership fees, sponsorships and consultations. 
The lab has set up a steering group composed of owners and investors plus representatives of city council and a few visitors. The steering committee is restricted to members who have financially or technologically supported the lab with valuable assets that further grow the lab. The operational structure of the governance system is simple consisting of a living lab manager, an FTE engineer expert, supporting resource from MIMMA InterExpo, and supported resources from E2. Phase 2 of the living lab will have a more sophisticated structure introducing networks of SMEs, research institutions, associations and more.

MIMMALL uses social networks and its website as key tools in its communication strategy. It also publishes articles in newspapers and magazines to gain access to traditional communication markets. It considers public visibility and critical success factor in the operations of its living lab.

The living lab uses the museum premises as a whole (auditorium, anteroom etc....) as its test best. This usage creates a real-life test environment where they can analyze the natural behavior of citizens' interaction with the technology or services. In this manner, the lab can convert its day-to-day visitors into co-creators of the technologies. The museum is equipped with plenty of multimedia and interactive technology such as TVs, projectors, interactive boards, AV control systems and a Simulation Lab. The lab is powered by high speed internet (Ethernet and wireless). The lab is networked over lan and managed through software that controls user registration, media content, and runs statistical analysis. MIMMALL hosts conferences, workshops, web-seminars and 
marketing campaigns as part of its lab activities. The biggest advantage an SME gains by using MIMMALL is the large and sophisticated test bed they own. The test bed allows producers to test their inventions in real-life scenarios under no controlled conditions to learn and understand the qualities and validity of their products.

By transforming the museum itself into the test bed, regular visitors and specific target groups get to participate in the co-creation process of the new technologies and services. This setup eliminates the barriers of the recruiting user. The user base can vary is background ranging from young children in schools to retired persons. All needs and requirements for innovation development comes from web based forums and visitor questionnaires. The users are educated about the technology through musicals and demonstrations and co-creation can be conducted through Wii games. Surveys and interviews provide feedback from the users after their interaction in the museum. The companies and users can co-design through the multimedia exhibitions or the online forums.

\section{A.29 Mobility Lab}

Mobility living lab is situated in San Sebastian, Spain. The lab seeks to be a leader in the optimization of resources regarding transport traffic in urban areas. Through the development of mobile solutions and services, the lab seeks to improve traffic and driving to reduce accidents and traffic jams. The lab manages models for vehicle flows and they develop technology to optimize such flows. They redesign transport resources and road infrastructure to create new services for the citizens. The lab uses its personal 
website to traffic information about its projects, promote its events and organize courses. A big portion of communication is done through word of mouth. Most members of the lab also host their own websites through which they too disseminate information about the lab. A project manager has been appointed to coordinate the operations of the lab. The project manager implements the projects and selects the members who are to participate in the process.

Researchers, technology suppliers, users, politicians and road authorities are all members of the lab. They work together to achieve advanced technology and control necessary data and optimize traffic. The owner is GAIA, an Electronic, IT and Telecommunication Industry Association. Drivers, governments and road authorities are the user who will be implementing the advanced technologies to make their experience more comfortable and safer. GAIA's consortium consisting of more than two hundred IT enterprises provides supporting roles in consulting and building the infrastructure. The consortium uses their access to markets and suppliers to the implementation of business solution. The council of Gipuzkoa and the Council of Bizkaia are financial investors in the lab and represent political needs. They approve or disapprove projects, do demands and set policies. Sponsors of the lab include Bidegi who manage roads. All members sign consortium agreements that limit the partners' ability to use, license and share the rights to the outputs of the labs. However, the lab treats each project independently and prepares IPR agreements for each. The members who benefit the most from the lab are the end users who get access to new technologies to make their driving experience better. Companies also benefit from validation of their product and establishing a market through the lab. 
The infrastructure of the living lab consists of buildings equipment with the tools to design and build the technology. The buildings also have the lab facilities to be able to run controlled tests. The living lab has set up advanced computer networks and tracking sensors connected over Internet to conduct their designs and tests. The methodological approach the lab uses is simple. The government councils and road managers express their needs to the lab explaining what they believe are required to optimize traffic. GAIA and its associated expert business partners find solutions to the different projects. The managers of the roads in conjunction with drivers test the new technologies in simulated and real-life environments. Finally when everything has been perfected, the companies can launch the innovations to commercial markets.

\section{A.30 OFF-ROAD MEMORY}

OFF-ROAD Memory Living lab is the largest user-centric innovation organization in Nantes, France. The lab's objective is to federate public-private collaboration to build a better touristic, economic and cultural heritage for the region through advancements in mobility, geolocation, geo-tagging, augmented reality and QR Codes. OFF-ROAD is dedicated to growing their knowledge economy and developing an innovation mediation method; they will use semantic web technologies and social media networking tools to conceive the goal. Overall, OFF-ROAD aims to exploit the common daily lives of its citizens and their digital habits to help innovate for the sake of raising life quality and solidarity of their community. 
OFF-ROAD are currently running several projects with local industrial partners for the development of new sociocultural practices, which are based on participative processes. These projects use a variety of multimedia content to generate a collective memory for the region. Most projects deploy QR codes into the surrounding region offering on-site access to digital content regarding the geographical site. The TAGDEVIN project is an augmented reality mobile application that uses QR code to link wine bottles to multimedia content promoting wine growing and new healthy practices. CARRE D'EMPREINTES is an inexpensive, green solution to the dissemination of tourist information through digital means (compared to paper flyers) through the use of a QR code that links multilingual media resources to the tourist. This project aims at creating a collective memory of the regions socio-territorial heritage through tagged objects/locations. VUES SUR LOIRE is a project developed in collaboration with TV broadcasting channels France 3 Centre and France 3 Ouest. The project defines a digital information sharing space for participative communities regarding the river Lorie patrimonial culture. Feedback has validated the projects and suggests that it they will generate a touristic economy; stimulate communication and socio-dynamic movements for the purposes of cultural and geographical initiatives.

The lab receives private funding from its industrial partners for public products such as the tag circuits for the growth of the socio-economic goals. They also receive public funding from the regions council for investment in private products and services that will aid the public. Most importantly, the lab creates its own revenue stream through the sales 
of its developed products and services. As a private company, the living lab can win commercial contracts, issue invoices and complete deliveries.

The lab has created a Steering Committee, consisting of the founding members, who federate the labs operations and hold the highest level of decision-making process. Project governance consists of a two levels of organizations; the Permanent Management Board (PMB) and Workshops. The PMB consists of a panel of public-private-people representatives and are responsible for fostering, collecting, diffusing and managing the labs "know-how". This level is bound by the rules and regulations of the labs objectives and resource portfolio. The second level, the Workshop, is an organized gathering inviting voluntary participation from project partners in order to identify needs and setting up collaborative projects using the labs framework.

OFF-ROAD uses an open collaboration mentality for its operations. Once partnerships are formed and members accepted, they are provided free access to the knowledge database that the lab has formulated. Under the Arexcpo-Ethnodoc knowledge base, the lab does restrict access to such information as religious funerals, intimate family anecdotes and others in respect of privacy for its members. Public content is governed under open-source licenses such as GPL while private and institutional sponsorships are covered under specific IPR policies.

The lab is structured as a Public-Private-People partnership (PPPP). As such, the lab consists of several stakeholders. CAPACITES SAS, a private local company affiliated 
with the University of Nantes, is the owner of the living lab and acts as the main Innovation unit. The University of Nantes involves its departments of Laboratoire d'Informatique de Nantes Atlantque and Compture Science Research Insitute, Department of Communication, Applied Foreign Languages Department, Polytech'Nantes (Graduate School of Engineering - Computer Science Department), Centre de recherche en littérature TLI-MMA - Welcome Team (EA 1164) and the Groupe d'Etude et de Recherche Scénologique en Architecture (GERSA), research team of the national graduate school of architecture of Nantes, for the purpose of innovation research. Likewise, the living lab engages international partners as well who are in the field of education for the increased support of research innovation. These stakeholders include: LAboratoire Franco-Mexicain d'Informatique et d'Automatique (LAFMIA UMI 3175 CNRS), Universidad De Las Americas Puebla (UDLAP - Puebla, Mexico), African Living Lab (ALL) and ISEG/UNIDAF group (Senegal). The living lab has both local and international technological partners: Atlantic 2.0 (regional innovation cluster federating more than 150 enterprises), France Télévisions, France 3 Région Centre, France 3 Région Ouest, Office National du Patrimoine Culturel Immatériel (OPCI), Ethnodoc-Arexcpo (Vendée), Pôle de Coopération des acteurs pour les Musiques Actuelles (Pays de La Loire), Territories of Tomorrow Foundation (Paris, France), Red Temática del CONACyT en Tecnologías de la Información y Comunicación (RedTIC, Mexico), the Comité de Informática de la Administración Pública, Estatal y Municipal A.C.(CIAPEM, Mexico), African Living Lab (ALL) and ISEG/UNIDAF group, Birds Living Lab and GAIA (Bizkaia - Spain). 
OFF-ROAD is dependent on mobile web technologies and smartphone capabilities to be able to deliver their multimedia for geolocated regions and integrate the users with social networking tools. Therefore the most important technologies required by the lab are: W3C Semantic Web Standards (RDF, SPARQL, OWL, and SKOS), W3C Web Design and Application Standards (HTML5, CSS, SVG, Ajax). For support of such technology, the lab also requires an infrastructure of $3 \mathrm{G}$ mobile networks, Internet mobile phones and web servers. The lab aims to deploy a social network tool that will help them observe their community's practices, knowledge and interests for future analysis.

OFF-ROAD uses its own website to post news updates, promote events and project agendas. However, the lab also utilizes other forms of communication including its partners broadcasting channels where they run a weekly program for twenty-six minutes. Other partnerships also offer their channels through the National Research Laboratories, Chamre de Commerce et d'Industrie and Societe d'Economie Mixte SEM Region. Of course the living lab capitalizes on self-promotion through their developed projects and interactivity with users and investors. OFF-ROAD also hosts training session at the Universities for students and seminars for organization for the purpose of educating and diffusing new technologies and promotion of events.

The lab hosts workshops with partners and volunteers to help identify the needs and requirements for setting up a project that solves a regional problem. OFF-ROAD supplies its users with methods and tools to enable them to produce content and give feedback on the new innovations being deployed in their region. The lab also helps create and foster 
user communities and produce editorial practices for TV, host events and also contribute to social media to help and encourage user participation. The lab promotes the production of multimedia content (videos, photos, songs etc....) in addition to the facts, stories and citizen information they add to the database. The lab sets the users in real world environments and harnesses their everyday life to build up their collective memory of the region. Citizens and tourists are the primary benefactors of the living labs operations. In return for their contribution of content that enriches the database and multimedia, they get free access to the remainder of the content generated by the labs community. Companies participating in the lab can tap into this user community to deploy their complementary products. Companies also can learn and use the labs framework of innovation to help them develop and validate their own innovative products while having access to support in marketing and communication.

\section{A.31 Pôle Numérique}

Pole Numerique is a living lab found in the Rhone-Alpes Region of France. The lab is adopting the progression of ICT into their territory and aims to build up the regions networks, services and data infrastructures to help facilitate open-innovation, citizen participation in co-creation activities and produce new economical models and governance regulations. The upgrade of the territory will collect actors together to form an ecosystem around new Web and digital capabilities helping in the management and the preservation of the territory. The lab itself will be a focal point of resources and experimentation to help members develop services, collect data, install infrastructures 
and set new standards in open-source and open-access of software and networks. Pole Living Lab leads the economic Commission of Arco latino Association (sixty-eight provinces from southern countries France, Italy and Spain. They help promote cocreation of open sets of data and help develop open services in the context of using readily available information.

The lab has a three-tier structure of governance that federates the activities and resource distributions of the lab. The first level is a Management Board, sets the mission and vision of the lab; it is composed of directors representing private corporations, policy makers and members of the public. The representatives are elected from business managers or civil society organizations. The lab's diverse board of directors allows for the consideration and incorporation of all member perspectives within the decision making process. The second level of management is the management group that is lead by an appointed director and governs the local and international projects, the labs community and is responsible for the economic evaluations of the activities. Lastly the third level of management consists of an advisory team and work force with councilors in areas of financial engineering, business and public issues workers and administrative helpers. Together, the three tiers coordinate the strategic orientation of the labs activities, projects and resource allocations.

The lab uses traditional media channels of local and national press primarily for the promotion of its events. All other forms of communication such as information publication or interactive discussions with its members are done through the Internet. 
They use their own website that hosts a blog; they are active on social media platforms such as Facebook, LinkedIn and Twitter. To expand their access to other forms of users, the lab will post their work on websites such as slideshare, and prezzi. In a similar manner, the lab allows for their web content to be downloaded and shared through rss feeds and so forth to maximize openness and communication.

There are over forty departments who are part of the lab including French stakeholders such as the state, Rhone-Alpes Region, and the Belle de Mai incubator. The more important stakeholders are: Department of the Drôme, ADN, Consular Chambers, RhoneAlley, the club of companies of the digital sector, ESISAR which is the National college of engineering in Advanced Systems and Networks, Valence and EPI. The following stakeholders who are involved are listed below with their respective role:

- Zenexity (French service provider): building open source web portal for citizen services

- AtReal (French service provider): developing open source suit of software for internal public bodies management

- Atol (French service provider): working on open source filing system for electronic document and legal archive

- Reply (Italian service provider): business and social networks

- Politecnico di Torino (Italian university): expert in cloud architecture

- Brunel University (Great Britain): expert in privacy and security 
- Blau Advisors (Spanish company): expert in change management and business model

- Engim San Paolo (Italian company): expert in training, communication and change management

- Provincia di Torino (Italy): pilot site for deployment and demonstration of the platform

- Comune di Bussoleno (Italy): pilot site

- East Marmara Development Agency (Turkey): pilot site

- David Holding (Bulgaria): service provider for business and public bodies and also pilot site at a national level

Pole living lab is currently dependent on financial support from public funds. It is in the process of being remodeled into a hybrid format to receive funds from both public and private organizations. Public funds can therefore be used to drive projects that serve the general public and more global interests, while private funding can be used in more specific activities. Projects undertaken for the territory should be supported via private partnerships. The lab welcomes new members and the support of all who are interested in building up the territory.

Pole uses a mix of bottom-up and top-down methodology during their research to maximize contribution from the members and not impede creativity. Diverse workshops are organized with artists, young people, civil society representatives and others to brainstorm and discuss problems and their solutions that the lab could work on. During 
the meeting, the participants access the pros and cons of the ideas to complete a hypothetical development process. The collected information is published and shared with the members of the lab and presented to the management teams for final decision. The lab also uses advanced tools such as virtual worlds or social intranets to improve communication for collaboration. Other tools are provided to help digitally design solutions and visualized on screen prior to contraction. The lab offers to its members the opportunity to explore new forms of innovation processes as well as access to experts. They offer financial engineering for project development and use their platform to bridge members together to collaborate on projects. Pole Numerique uses open and common creative licenses such as GPL or the CC-BY-NC to manage their IPR.

\section{A.32 Regional strategic impact through open innovation and creative use of ICT}

\section{(LLMidt)}

LLMidt is a network of living labs physically situated in different location and whose sector research focus varies. As such, this living lab has an area of research domains including health, tourist entertainment, smart cities for transportation, food and agriculture, energy and environment. I idea is to create model for future sustainability of the societies welfare for the region. The lab will test, with the help of government and business, various new technologies and business models that will be founded in usercentered design. Using the progress made in ICT, LLMidt will investigate each domain asking such questions as: "Can ICT deal with an increasing number of elderly people? How does ICT transform the transparency of governance? Can ICT transform the very 
traditional tourism sector in a new wave of economic stimulation? How will ICT help in managing utilities? How can ICT stimulate and enforce new innovative value chains across branch and sectors?” The lab adopts concepts from bottom-up structure and involves the user heavily in the innovation process. The lab empowers the users during workshops to drive idea generation and help co-develop with the industry. Users are so involved that home visits; public meetings and educational initiatives are not uncommon during the innovation cycle of the lab.

The living lab has a formal governance structure consisting of a Coordinator, Secretariat, Steering Committee, and an Advisory Board. The Coordinator oversees the overall living lab leading the Secretariat and the Steering Committee. The position is also responsible for maintaining good relations with ENoLL. The Secretariat conducts the daily operations of the living lab engaging participants to start up new initiatives, conducts evaluations of established locations, and directing the administrative tasks of the lab. The Steering Committee is responsible for the strategic operations of the lab ensuring its activities meet the goals and mission. Lastly, the Advisory board is to provide direction to the labs decisions and operations based on international research and position. The Coordinator is made up of representatives from the Region Midt, the Secretariat is composed of Region Midt and Arhus University, and the Steering Committee of research centers, living lab hosts and industry members. The Advisory board is yet to be determined but most likely will consist of representatives from ENoLL. The Steering Committee makes the decisions with consensus agreement. If a participant feels the decision will impact their function or organization, the participant may veto the decision. When consensus is not reached, the 
decision may turn to a majority vote selection, to move the process along. LLMidt handles all Intellectual Property Rights issues on a case-by-case situation. All joint IPs that have been developed through the lab is controlled through the shared agreement. However, the original IPs contributed by the industry members remain the property of those members and shall be exercised according to their will.

The main stakeholders of the living lab are the Central Region of Denmark, the City of Arhus, SkiveET, the Alexandra Institute and Arhus University, as well as Center for Digital Urban Living (DUL). They are a well functioning team having a long-term research and development relationship. DUL especially has been initiating and coordinating many projects for the exploration of experiences in public space. Likewise, Egmont Hoejskolen, a boarding school for disabled students, have much experience in exploring assistive technologies in the healthcare field.

The lab currently runs off European funding but seeks to gain access to local funding. The lab does not retain its own infrastructure to operate the living lab, however, with strong ties with the government and local partners, it is easy to coordinate the use of facilities. LLMidt provides knowledge to government enabling them to develop new policies and strategies for public-private partnerships. The run the "At a glance" project where the living lab provides overall communication and coordination during the festival related to health security. The lab also is working on SMART Region project where they combine urban development with the digital economy. The project aims to buildup the ICT infrastructure to establish an ecosystem between government, citizens and business to solve the problems of society. 


\section{A.33 SaMPL Cultural and Creative Living Lab}

SaMPL living lab is an Italian ecosystem established in 2010 around boosting creative economy and cultural heritage. Intended to be a conservatory for Padova, SaMPL uses the living lab approach with integrated laboratory to research the use of ICT to maintain the cultural heritage and grow the arts, media and functional music production. The lab's aim at boosting local economy via its heritage is based on the exploration of new business models for IP management, licensing agreement and joint venture for market expansion. The lab itself will act as a center for knowledge sharing, collaboration and business development. It uses its resources and support of skilled researchers, entrepreneurs, facilities, users, and public representatives to progressively undertake projects and build SMEs. The lab will bridge the economic development with cultural heritage aiming to maintain stakeholder's satisfaction and growth in the region with fair distribution and rewards.

As an ecosystem, the lab acts as a point of convergence for the members allowing for cross-formalization of ideas, information exchange and business networking. They have in place a technology transfer plan to help move ideas of one member to commercialization through another member to facilitate business generation and growth. Likewise, the labs defined framework for collaborative work, complemented with the networking tools and development technologies, creates a suitable environment for companies and others to achieve innovation R\&D. Lastly, because of their extensive 
operations, the lab has undertaken several projects and initiatives that already legitimize members who now join and add value to the labs work.

SaMPL lab has many channels of communication. Their projects, tutorial sessions and music concerts are self-promoters, but the lab still uses many free social media outlets such as Facebook and Twitter as well as its website. Other free software tools such as source control systems and material reservation, tracking systems, and event calendars are used for organized communication and management. It is through the use of these free software that the lab is able to fully achieve the openness of a living lab and bring stakeholders together to collaborative purposes.

SaMPL is continuously developing their governance to better manage its complex activities. The conservatory of Padova and the Department of Information Engineering of the University of Padova control SaMPL governance, incorporating and respecting the input of fellow stakeholders. The involved stakeholders and a group of competent experts manage each initiative and project. A supervising committee with various individuals from different backgrounds helps coordinate the projects. SaMPL uses a bottom-up approach as part of their creativity generating process in their open innovation process. However, the lab also applies a top-down approach to ensure all organization and a controlled co-creation process. In this manner, SaMPL has created a hybrid model to help keep creativity alive in the process without losing the research methodology of a living lab. As an open innovation living lab, SaMPL applies GNU public license for all the software developed by their organization, and a Creative Commons BY-SA license for 
text, media and other content produced in-house. The lab still respects other licensing schemes and will adopt them as needed to help maintain an open source and open innovation environment.

The public investments from Fondazione Cassa di Risparmio di Padova e Rovigo and from the members currently fund the lab. SaMPL has invested significant effort into fund-raising initiatives and is investigating alternative sources such as revenue models to make the lab self-sustaining. In order to effectively divide the cost between actors and reward stakeholder contributions, the lab will sign partnership agreement for licensing of product and services to allow for profit making opportunities.

SaMPL Lab currently has over seventeen members and six hundred employees with user categories ranging from artists to regular citizens. Public institutions, technology transfer centers and business associations are among the stakeholders of SaMPL. The corporations involved in the lab ranging from SMEs to large enterprises are:

Comodamente Festival, Fondazione Ghirardi, Amici della Musica di Padova, Laboratorio Arazzi, Fondazione Cini, Progetto Giovani del Comune di Padova. Sponsors of the lab who help promote and finance operations are: Fondazione Cassa di Risparmio di Padova e Rovigo. Research institutions are among the most prevalent having the following on board: University of Padova, The Department of Information Engineering (DEI), Sound and Music Computing group, Intelligent Robotics group, Artificial Intelligence group, Aalborg University in Copenhagen, Middlesex University London. Together, the 
stakeholders make a consortium of expertise in marketing, digital content, media, education, engineering, radio, TV, and other ICT capabilities.

Because of the structure and nature of the work of the lab, it does not require a localized infrastructure to conduct operations. Instead, the lab uses various buildings and assets as provided by its members including:

- hardware laboratories (about 120 square meters) are supplied by the Department of Information Engineering.

- haptic feedback devices;

- optical systems for full-body motion capture;

- sensors; multi-channel audio diffusion system;

- silent booth ( $2 \times 2 \mathrm{~m})$ for controlled audio recordings;

- DSP development boards

- ICT provided by Department of Information Engineering

- public spaces, including the main Concert Hall: used for public events and for the display of interactive installations

The lab predicts that in the near future, these resources will be consolidated under one roof making a focal point for structure hosting, laboratories, test beds, meeting locations and public spaces. 
SaMPL try's to involve the users in all aspects of the product cycle; creation, design, production, promoter and receiver of there own innovations. Engaging the user comes from the publically hosted events where the lab invites citizens to learn and participate in the projects of the lab. The lab will use focus group activities and idea competitions to encourage contribution. While users provide ideas, it is often the researchers who bring technical knowledge and enterprise members adding market trend information. Together, in a workshop, the collage of members can brainstorm solutions to be developed with state-of-the-art technology, with quality feedback and commercial viability. Decisions are voted upon as a team, and executed by those who wish to carry through with the project. At the end, companies are the ones who try to commercialize the innovation for the purpose of dissemination and exploitation. 


\section{A.34 Sheffield City Region}

The Barnsley living lab of the Sheffield City Region (SCR) in the United Kingdom is public-private partnership that aims to bring next generation broadband to the region. It is an initiative that is supported by the UK national Go On campaign. The lab recognizes the economic and social benefits of ICT and wishes to grant access to the vast majority with the provisions of e-services and to support SMEs in the digital sectors. Through the ICT development, the lab hopes to expand and apply its ICT to Smart Cities, Social Innovation, e-Government, Creative Media, and Logistic Development.

There is a change in the governance of the living lab. South Yorkshire, a previously administrative county, has led the digital agenda and e-Forum since 2001 while still incorporating the local authorities, universities, health, police, fire and rescue service and regional development agency. As such, despite the governance transition, the management is semi-formal maintaining an open and collaborative mode. Members of the lab can use the e-Forum to participate in meetings while under the constraints of procurement law. The Forum is used as a strategic framework and is now being re-shaped to encompass a larger region, geographical and economic digital agenda. LEP, a private sector led board, has assigned board members (co-founder of Plusnest and city councilors) to lead the work on the digital agenda. The regions local authorities, in partnership with the universities, meet regularly to promote strategic collaboration, identify shared problems and opportunities, and nurture the network. SCR is assembling a team of four people who facilitate and implement LEP's plans, data centers and 
collaboration ecosystem. ERDF, the funding organization of the lab, is creating a small coordinating/networking team that will help the lab to develop knowledge communities across South Yorkshire.

There are several stakeholders involved the lab who contribute both physical and nontangible assets.

- The South Yorkshire Public Sector e-Forum: Brings together the public and private sectors together for the cause of the digital revolution. They provide horizon scanning of technological developments

- Making IT Personal: Trains and accredits digital mentors while seeding them throughout the community

- Sheffield University and Sheffield Hallam University: Conducts research, knowledge transfer and outputs spin-off companies and offers top class facilities, idea generation and key personnel

- Sheffield City Region Local Enterprise Partnership Board: Provides strategic direction for the economy

- Local Authorities: Provides democratic legitimacy and financial resources. The public services supply problem requisitions for which the living lab must develop solutions

- The Regional Development Agency: Conducts economic forecasting, coordination and financial resources 
- e@syConnectss: Provides the mechanisms for problem solving and deployment of services

- SMEs: companies including Town Talk, Epsilon, Bull and Thales. They created Digital Region Ltd a special purpose vehicle to deliver the next generation broadband.

A real test-bed is used to implement solutions and applications that can be tested in reallife scenarios with a wide range of demographics through the labs ecosystem. Most of the labs resources are diffused throughout the region and is present in the member's facilities. SCR utilizes next generation broadband as part of its infrastructure to be able to supply etechnologies to the citizens and create digital inclusion. They also provide next generation wholesale data center campuses with public sector automation and transformation.

SCR uses new web technologies to create a new and interactive communication channel to create a two-way medium that allows for effective exchange of information between members, the public and the lab. The lab has built a two-way conduit between citizens and service providers. Through the use of new web standards, the lab is able to encourage participation with real-time comments, co-design and delivery. Together they provide ideas and solutions to develop the new and innovative technologies sought by the lab. Together, they also provide the necessary environments and tools to build and test the innovations. Businesses provide the tools and design infrastructure, while users provide the real-life test environments. Users are consulted prior to a project to gain their 
knowledge and input on needs, during the project for testing purposes and after the project for feedback. Businesses are responsible for the development of the solutions. They act as local drivers for innovation by contributing innovations and ideas but most importantly the motivation to push for new technologies. Members of the lab can expect to gain access to expert personnel, local and incoming business and an array of other participants through the labs ecosystem. Entrepreneurs can use the provided incubation space to help propel their business activities and request support from the expertise provided in business, marketing, communication and IT specialists.

\section{A.35 SPORTIS Living Lab}

SPORTIS is a living lab found in San Sebastian, Spain. The lab is a co-creation environment for innovation of the local, national and European sports sector. As part of INESPORT, the lab seeks to participate in the decision making process of the sports industry at a national level. SPORTIS intent is to implement projects within a broad scope of sports market, covering very different competitive activities and demands such as health and wellness. With the help of companies, public administrations, research centers and citizens, the lab will conduct innovation co-creation. As such, the lab takes on the structure of a public-private-people partnership. The lab itself will make project propositions, define requirements and coordinate the activities of product validation. SPORTIS covers all aspects of the value chain. It involves development process, software development and business development. The lab expects to use wireless technologies and sensors and other technologies such as microencapsulation to achieve these innovations. 
SPORTIS advocates for the creation of a community of knowledge in sport. It seeks to use a network of partners and collaborators to promote projects. It promotes the creation and sustaining of partnerships and relationships, promotes collaboration on different projects, and access to information and know-how knowledge.

SPORTIS decision-making governance is composed of the driving committee that has two levels of organization. The first level is the permanent management board, which is nominated by the driving committee depending on the occasional needs and activities of the lab. Second level is workshops, which are organized around the fields of application and are responsible for identifying and setting up user-centric, collaborative projects. Self-promotion is evident through the developed projects and the support of its users and investors. However, the lab officially uses its own website to communicate its activities and furthers such activities through organized events and courses in collaboration with the University. National and international organizations that are aligned with the lab also help promote the lab their sites and events. Nonetheless, the lab still uses traditional forms of communication such as media publication to advertise their events and promote the lab.

It is the labs belief that open and collaborative attitudes are what empower the innovation process. The lab there for opens its consortium to meetings, workshops and events making all information available via newsletters and web page. Partners have access to all information including patent research, technologies, market, information activities and publications and other research. Likewise, the labs consortium is open to accepting new 
partners who are interested in participating in the labs undertakings given they contribute value to the projects. SPORTIS governs the IPR principles through a signed consortium agreement that point out the allowances and limitations of use of the IP and knowledge sharing. In short, the IP agreement is that knowledge is to be shared, mutual access be made available to a high number of commercial products and provide licensing of development.

Figure 24 below shows the stakeholders involved in SPORTIS consortium and Figure 25 describes their contribution to the lab.

\begin{tabular}{|c|c|}
\hline Areas of Speciality & Users/ Partners \\
\hline Universities & $\begin{array}{l}\text { Universidad de Deusto, Universidad del } \\
\text { País Vasco, ESTIA, Francia. }\end{array}$ \\
\hline Technological Centres & Instituto Biomecánico de Valencia, \\
\hline Business creation and strengthening & Jausas Capital Riesgo \\
\hline Telecommunications and Technology & GAIA companies $(300)$ \\
\hline SPORTS & INNESPORT \\
\hline Companies & $\begin{array}{l}\text { Orbea, Import Arrasate, MONDO, } \\
\text { Innovatecs, Barrabes, Intersport, } \\
\text { SportCreber, Philips, D'appolonia }\end{array}$ \\
\hline
\end{tabular}

Figure 24 Stakeholders of SPORTIS (ENoLL, 2014) 


\begin{tabular}{|l|l|l|}
\hline Contacts/support & Identification & Type of support or collaboration \\
\hline Investors & Individuals & Knowledge and networks \\
\hline Suppliers & $\begin{array}{l}\text { Companies in the ICT sector, universities } \\
\text { and technology centres }\end{array}$ & Technological developments \\
\hline Customers & $\begin{array}{l}\text { Sports institutions, sports distributors, end } \\
\text { customer (fond of the sport/physical activity) }\end{array}$ & \\
\hline $\begin{array}{l}\text { Technological contributors } \\
\text { / partners }\end{array}$ & $\begin{array}{l}\text { Innovatec, University of Deusto } \\
\text { Import Arrasate (Astore, Temua), Orbea, IBV } \\
\text { and Gaia }\end{array}$ & $\begin{array}{l}\text { Technological developments. Technical } \\
\text { support to the different projects } \\
\text { developed }\end{array}$ \\
\hline Enterprise Network & Inesport & $\begin{array}{l}\text { Search of partners in projects and } \\
\text { dissemination of them }\end{array}$ \\
\hline Clusters & GAIA & $\begin{array}{l}\text { Intercluster relationships in order to } \\
\text { identify synergies and other areas of } \\
\text { collaboration and promoted technological } \\
\text { developments application }\end{array}$ \\
\hline Associations/groupings & GAIA & $\begin{array}{l}\text { Tutoring of entrepreneurship support. } \\
\text { Support the establishment of } \\
\text { collaborations between SPORTIS and } \\
\text { other companies in the ICT, both in the } \\
\text { Basque country, and at national and } \\
\text { international level. }\end{array}$ \\
\hline
\end{tabular}

Figure 25 Value Added by Stakeholders of SPROTIS (ENoLL, 2014)

SPORTIS requires three types of technology to proceed with innovation in the sports market, micro-encapsulation, miniaturized sensors and wireless technology. Microencapsulation is a technology that produces microcapsules and than attached to other materials. Miniaturized sensors allows for picking up increasingly more information in difficult to reach places. It can be used to track elements in space and register parameters. Lastly wireless technology enables the transmission of data between the products and smart phones for user ability. 
SPORTIS involves users in the development and validation of new products. They are also involved in determine the uses of the product while expressing their perspectives and attitudes towards them. The lab therefore considers that the users are involved in the whole value chain of development through their active participation including the partaking of summer courses. The summer courses are intended to not only educate the users but also act as a focus group where members can share ideas. The lab uses an open platform to source ideas and suggestions from its members. They discuss innovation, certification and infrastructure and technology within the sports field. The knowledge generated from the users is left open so subsequent developers can further build open it. The lab uses ObesiTIC as a way to perform reality checks and trials on the developed products. SPORTIS offers many benefits to its members. As a living lab, it can benefit companies and entrepreneurs through its activities of trend watching or user need finding. Additionally, the lab provides methods and tools that help the design process, testing and validation during workshop activities. Participants also get visibility for their brand and recognition from the European countries for their contribution to research and development. SMEs therefore also get the opportunity to network with other business through the consortium the lab has established. In total, the lab provides an opportune way to fast-track innovation and business development for its members.

\section{A.36 Telecommunication Networks and integrated Services Living Lab}

Telecommunication Networks and Integrated Services living lab (TNS LL) is a Greek lab that focuses on researching cognitive/autonomous management, convergence, and Future 
Internet technologies, Composite Wireless Access, Fixed Access/Core Networks, Sensor Networks and Software Defined Radio. The lab aims to conduct research and development in these fields as well as offer complementary educational activities and consulting services. TNS LL conducts R\&D in all areas of telecommunication networks and services. Additionally, the lab actively provides educational and consultation services towards its members including government ministries and academia.

The senior members of the living lab are the governing entity that makes the strategic decisions for the TNS. The senior members do consult and collaborate with participating members before making decisions, but such collaboration is weighted based on the level of involvement by the fellow participants. The lab uses web portals as its main tool for communication with its members. It is through the web page that TNS LL can publicize information regarding $R \& D$ projects, update topic details, and promote and share its events. The web page allows for monitoring of the members hence also acting as a management tool for the TNS LL.

TNS respects and honors member's IPR. Depending on the level of R\&D material and the context within the project is being developed; company IP will be respected and the lab will act according to the Grant agreement provided by its members.

TNS LL has established a network of stakeholders, similar entities, organizations, and partners to foster collaborations and open dialogue for innovation. The network includes Worldwide Universities Network Cognitive Communications Consortium (WUN 
CogCom), Wireless World Research Forum (WWRF), Working Group 6 (WG6) and Wireless Innovation Forum (SDRF). All stakeholders of the lab contribute assets and capabilities that help grow and operate the living lab. The labs owners, the public sector in partnership with the University of Piraeus, provides utilizes such as buildings, offices, power and telephone services. The European commission, public and private companies of Greece, are sponsors who provide funding through out the R\&D activities. TNS LL partners bring together the opportunity to collaborate on innovation projects and exchange knowledge for the evolution of their expertise and the accomplishments of the projects. These partners come from different backgrounds and include Alcatel-Lucent Bell-Labs Deutschland, Telefonica Investigacion y Desarrollo S.A. Unipersonal, Thales Communications S.A., Intel Mobile Communications, NEC Technologies UK, VTT Technical Research Centre of Finland, EIT+ Wroclaw Research Centre, University of Surrey, Universitat Politecnica de Catalunya, La Citadelle Inzenjering, Bundesnetzagentur fur Elektrizitat, Gas, Telekommunikation, Post und Eisenbahnen. Lastly, the lab also has stakeholder users composed of powerful organizations with rich, worldwide experiences and multilevel expertise. These users provide their effort and expertise to fulfill the goal of the labs projects.

TNS LL performs their research directly with users and physical communication channels. The products to be tested and validated are applied in real-life environments to maintain realism and important life variables. The perks of being a member of the lab is the opportunity to take advantage of the ecosystem the lab has built and the expertise it has pooled together. Members can access the professional services the lab has to offer in 
order to enhance their organization and offerings to their consumers. Likewise, the lab provides an environment, in which the members can be encouraged and supported to evolve their knowledge and expertise, expanding their professional horizon and network with national and international partners. Lastly, the members also have access to the knowledge and data that TNS LL develops during the research projects they conduct.

TNS LL has structured its financial model around its own resources in order not to burden the national budget of Greece. Its model and financial sustainability is based on its $R \& D$ projects, which receive funding from EU under FP7 and its respective members involved in the project. The lab also produces its own revenue stream through consulting services in technical matters and project administration offered to the Ministry of Education and Ministry of Finance. The lab offers other services including design, management and performance evaluation, software engineering, optimization techniques, algorithms and complexity theory as well as queuing theory.

Its owners provide most of the infrastructure of the lab including the building and office space. The equipment the lab already possesses is computers, laptops and consumables, and software programs and the devices. This equipment is used for education and research purposes. As part of its main objective, the lab has acquired access to composite wireless access, fixed access/core networks, opportunistic/sensor networked and Software defined radio. These include switches, VoIP gateways, Network Management systems, WiMAX base stations, subscriber units, PDAs with GPS, 3G, EDGE, GPRS, WLAN and Bluetooth support, Tablet PCs with WLAN and Servers. 


\section{A.37 Tepebasi 'Future Living' Lab}

The Turkish Future Living LL (TFLL) aims to improving its citizen's lifestyles through solutions targeting their social needs. The lab focuses on improving social and cultural life in the region through their output in social innovations, social enterprise spinouts and the building of social networks. The main expectation is to grow the digital inclusion paradigm that plays a role in social inclusion; the lab will provide Public Internet Access Points and public libraries, and digital technology to Third Sector organizations (social workers and NGOs) to help increase digital literacy and establish eLearning platforms or employment portals to its citizens. Through the living lab framework, Future Living will conduction innovation analysis and validation, help promote the use of ICT for socioeconomic development and positively impact the groups of disabled people suffering from mental or physical illness. Additionally, the lab will assess new business models that will enable the mass use of ICT or other technologies that will help achieve the labs mission in building socio-economic region and helping its citizens. TFLL's vision is to develop a creative, supportive and motivating network for its citizens and towns that are tackling health inequalities and striving to put health improvement at the core of all local policies. The value comes from the large-scale activities and projects that the lab get involved in with different public services.

TFLL uses a democratic governance structure to federate the labs operations and allocation of resources. This democratic structure includes all living lab members, and 
decisions are made together including negotiations and accommodations between parties. The mayor of Tepebasi who leads the committee of telecom providers, universities, technology suppliers and other entities chairs the lab. As a social endeavor, the lab has appointed an advisory board consisting of different groups of students and 11 social inclusion subjects to represent the cities needs. The lab also uses the advisory board to help reach young people and to develop their social skills through the participation of various living lab activities. Through the advisory board, TFLL will be able to connect young people with the right guidance and users to help build a knowledge society and improve their skills and facilities to offer innovations.

The lab acts as a social firm to employ the disadvantaged citizens. The lab targets deprived and unemployed young people, children living/working in streets, disabled people, old people, women or men who need protection or financial help. TFLL hosts meetings for women to talk with each other and tap into experts who can provide advice to their questions. The lab tries to provide an environment where hierarchy is minimized as much as possible and help stimulate entrepreneurship, community and individual growth. TFLL works with Rainbow Café to employ mentally disabled people and Ozdilek Art Center targets children and women who need financial help. TFLL also offers Neighborhood Houses to housewives who need social support and life long learning seminars. TFLL involves citizens in its various innovation activities to help build social inclusion and social entrepreneurship. The lab engages youth from universities and youth centers to harness their creative minds and instill the labs missions and goals in the new generation of Turkish citizens. 
TFLL has invested heavily in its continuous and open communication channels to maintain the flow of information between themselves and the members. The lab has lunched its own and new website in addition to their current presence on social media platforms such as Facebook and YouTube.

The stakeholders of the lab include universities, national and international enterprises and government authorities. Thanks to the remolding of the social initiative of the government into the living lab framework, the network of members has grown. The lab has approximately five thousand users by its local centers and another four thousand alternate centers. Below is a list of partners that the lab has networked into its operations:

- $\quad$ System partners: Turkcell and TTNET

- Component partners: Sampas AS (smart city automation system)

- Application partners: Internal IT department and Sampas AS (web and applications)

- Operating partners: Youth Center, Sport and Cultural Association of the Municipality of Tepebasi , Özdilek Art Center, 23 Nisan Child Center, Sukurusu Implementation Center

- Impulse partners: Municipality of Tepebasi Public service houses, Art and Cultural CenterResearch and innovation partners: Anadolu University

- Osmangazi University

- Civil organizations: Union of Chambers of Turkish Engineers and Architects (TMMOB), Turkish Medical Association (TTB) 
- Union of Turkish Bar Associations (BARO).

- Voluntary organization \& people: Community Volunteers Foundation (TOG), Active Philosophy (Aktiffelsefe), Experiental Education Center (DeM)

- National: Union of Healthy Cities, Union of Historical Cities, Union of Municipalities of Turkey

- International: Forum Virium - Helsinki, Arabianta - Helsinki, Culminatum Innovation- Helsinki, Living Labs in Malta, Switzerland, Hungary and Swiss Media Association, SuisseGreen Association, eWASTE Green NGO - Hungary

TFLL has not established any special IPR policies or information control mechanisms as all apps and outputs are open. The lab focuses its policy making on social municipal work and increasing awareness of being urban dwellers. TFLL does recognize that traditional management techniques are ineffective and require remodeling.

Funding for TFLL comes from both public and private entities. The Tepebasi Municipality primarily supports the lab. The municipality has sponsored the labs infrastructure through the investment and development of $\mathrm{Wi}-\mathrm{Fi}$ networks, multiservice fiber network, central VoIP, three hundred and fourteen PCs, nineteen Laptops, twelve servers, sixteen virtual servers, seventy-five security cameras and forty-seven thin clients.

The biggest advantage of participating in the living lab is the chance to be part of new social enterprises, which is a whole new class of business types for turkey. The lab provides direct Internet experiences to the citizens in public location. The lab helps with 
information literacy and offers education to the members; it uses instructional games as a teaching method and encourages the creation of music and other media. As an open innovation platform, the lab allows the transfer of information from the lab to community sites. The lab provides to its organization members up-to-date knowledge for use in the classroom.

\section{A.38 Territorial Living Lab Prealpe (TLL Prealpe)}

The Italian Territorial Living lab (TLL) is a consortium of companies with the goal of stimulating the innovation production in the Pedemonntana Veneta area. The motivation came as a response to the changing climate of technologies and the need to update the production districts so that they may continue to play an important role in the community and maintain the economy. TLL has adopted a platform like mode to its operations defining key characteristics to its intentions and operations. It will raise awareness towards ICT and support a user-driven innovation processes using open and sustainable economic methodologies, which favor participation and experimentation in real-life scenarios. The lab works with extensive and diverse of a partnership that involves stakeholders in an open collaborative agreement. It involves local community, public institutions and private companies steering the economic and social systems to improve the capacity the region to compete at the international level.

TLL has launched four different initiatives: eBusiness Lab, eLocGov Lab, MLab, TURidea. eBusiness Lab develops new ICT services for companies, through the 
operations of the companies. MLab develops Mobile platforms. eLocGov launches ICT services for public administration by conducting tests within town councils. TURidea, further extends the ICT technology to rural areas and sustainable tourism. TLL uses their own web portal in addition to promotional advertisements through TV channels.

TLL believes in openness as a means to encourage collaboration where all members in the ecosystem con contribute to the learning capacity of the lab. Their extensive partnership with the members is outlined in the collaboration agreement that all members signed. This agreement also outlines the IPR rules and confidentiality of the innovations and results.

Several stakeholders have become networked into the lab including local administrators of Bassano del Grappa, companies and their organizations, user groups and single citizens. The more notable participants (to name a few) are:

- Consorzio Di Servizi Pedemontano (Co.Se.Pe): A group of fifteen SME companies

- Fineco Innovazione: A service company

- Metae: A communication and brand company

- Tamerici: PR and Communication company

- Itabc CNR: National Committee for Research 
TLL sources its support from its public and private partners be it financial or sponsored equipment. The lab has a private business model to create revenue from innovation services it offers covering the production and operation costs. Consorzio di Servizi Pedemontano (Co.Se.Pe) uses its own permanent staff to support a management of the TLL. TLL's main ICT infrastructure is in the possession of Co.Se.Pe where they manage the web services that facilitate the participation of the members during the co-creation process. As Co.Se.Pe is a group of various companies, the ICT infrastructure and services are also distributed among them. TLL also takes stock in its natural landscape of mountains, hills and rivers in conjunction with its cultural resources (castles, archeology etc....).

TLL has access to over fifteen thousand users on their web-TV platform. The labs innovation methodology engages users in the beginning of the cycle by collecting their knowledge through their comments, opinions and feedbacks that is passed to the "characters". "Designers" likewise contribute their experiences and knowledge to the lab expressing the capabilities and limitations. Characters take the collected information and iterate through a cycles of refinement where the proposals are perfected prior to designing the co-design process. The co-design process is developed in the presence of companies and public service to ensure acceptable work procedures. Finally, "Enablers" conduct the innovation development process using the living lab framework using best practices. The lab uses its own ICT resources to monitor lab activity such as its public database, which is now becoming a growing resource for the territory. The ICT resource 
is a tool used by the members to interconnect the community during the co-creation process.

\section{A.39 Urban Living Lab - Versailles Saint-Quentin-en-Yvelines}

Urban Living Lab is partially located in the heart of the new and old city Versailles, France. The lab generally focuses on the development of new business models; however, it does so within the innovation fields of energy efficiency, mobility and food training. After establishing itself in such fields, it wishes to expand into telemedicine and human services. As a living lab, it actively promotes and nurtures a community of users harnessing their knowledge to better understand the territory and to develop ideas to better the city. It has already established an ecosystem involving students, residents, local communities, associations and business for the purposes of innovation exploration. This ecosystem shares their knowledge through their feedback and experiences and their implementation modalities. Together, they set forth new standards; develop best practices, new legislation and better the quality of living. This open innovation ecosystem supports the development of Eco districts and low carbon cities. The lab conducts three main activities to support the innovation ecosystem. Firstly, it investigates legal aspects by exploring the current legal barriers and contemplates best practices that would help drive change in the law. It also conducts studies on socio-cultural aspects in regards to transportation and city development based on the various social and cultural backgrounds

of the regions. Lastly, they explore new business models for the generation of revenue for 
products and services developed in the field of data processing, energy efficiency and multi-modality.

Currently, Urban living lab uses its own website to host and dissemination vital information about its activities and members. The website carries a database and portfolio of the collaborative works of the living lab. Social media technology such as Facebook and twitter or LinkedIn is also utilized to more extensively connect the members together. Local media such as news publishers, radio station and TV channels have also supported the lab and promoted its activities through their channels.

Urban Living lab favors an open innovation model and encourages co-creation activities. It works hard to maintain the ecosystem for the substance of collective intelligence. Because of the complexities involved in co-creation, the lab has to deal with ever intellectual property on an individual bases considering the type of product, service and member contribution.

The lab has different public and private stakeholders each contributing to the collective efforts of the lab. These public-private partnerships have the potential of defining new jobs in the Green Growth. The following parties are members of the lab:

- Fondaterra (owns and operates the Urban Living Lab project) 
- Higher education and research institutions: The University of Versailles SaintQuentin-en-Yvelines, Ecole Nationale Supérieure d'Architecture of Versailles, Institut Supérieur de l'Environnement, Ecole Nationale Supérieure du Paysage

- 2 districts : Communauté d'Agglomération de Saint-Quentin-en-Yvelines and Versailles Grand Parc

- SMEs : the cluster of companies « le Vivant et la Ville », DeltaSQY, Senda, Terra in Design, Avob, Greencove, Deways, Sinovia, Elan, Bertin technologies, Silicom, Smart Impulseo ONGs / Associations

- Students clubs (Faculté d'Agir), AFNET, Angenius, Complexcité, AgroEDI Europe, Réseau Européen des Villes Numériques, Fondation des Territoires de Demain, Club de Budapest, P3M (Planète du $3^{\text {ème }}$ Millénaire), CNFPT

Urban living lab adopts two forms of financial sustainability, public and private funding and a private business model. The lab uses funding from public and private sources to support project research and product demonstrations. The funding can also be used to cover market launch attempts, sponsoring project proposals searches, call for ideas and development of prototyping. However, the lab also creates a revenue stream through the data mining and the development of business models.

Urban living lab current infrastructure is the Versailles Grand parc business incubator that has fifty-eight cells dedicated to the hosting of the labs activities. This location has been deployed with advanced optical fiber network connections for high-speed 
applications. The lab is also investing into the future site of Satory to make an experimental site of new low carbon transports i.e. electrical trains and city powered traffic. The lab tries to involve all the stakeholders in the entire innovation chain. The lab will collect the needs, specifications and requirements from the ecosystem, and then assign the work to the students at the University of Fondaterra. The work is assigned through the prospective studies. The projects are then relayed back to community through the exhibitions, collective works and on the labs websites. The local authorities help its development by increasing the employment and resilience of the territory.

As a member of the living lab, major companies can expect several benefits including: the integration of best practices in their new innovative product or service and access to local markets encompassing local authorities and citizens. Members can expect support from the ecosystem, be it complementary technology or financial investment. Companies also gain the advantage of having their products and services tested and validated by real consumers prior to dissemination. 


\section{Appendix B}

Table 9 Objective Data points Assorted into Respective Categories

\begin{tabular}{|c|c|c|c|c|c|c|c|c|}
\hline & & Social & Business & Economic & User & & Test & \\
\hline Phrase & Collaboration & Impact & Development & Development & Impact & ICT & Bed & Framework \\
\hline \multicolumn{9}{|l|}{ implementation of various } \\
\hline \multicolumn{9}{|l|}{ activities that have been } \\
\hline \multicolumn{9}{|l|}{ conceived by the needs of the } \\
\hline people and the environment. & $x$ & & & & & & & \\
\hline \multicolumn{9}{|l|}{ The platform provides a } \\
\hline \multicolumn{9}{|l|}{ methodology to for innovative } \\
\hline co-creation. & $x$ & & & & & & & \\
\hline \multicolumn{9}{|l|}{ encourages the exchange of } \\
\hline \multicolumn{9}{|l|}{ ideas of between the various } \\
\hline types of business. & $x$ & & & & & & & \\
\hline \multicolumn{9}{|l|}{ incorporates infrastructures, } \\
\hline users, and various services. & $x$ & & & & & & & \\
\hline \multicolumn{9}{|l|}{ research approaches through } \\
\hline \multicolumn{9}{|l|}{ collaboration with other } \\
\hline \multicolumn{9}{|l|}{ laboratories in different fields } \\
\hline \multicolumn{9}{|l|}{ such as neuroscience, } \\
\hline sociology and philosophy. & $x$ & & & & & & & \\
\hline \multicolumn{9}{|l|}{ to identify and share best } \\
\hline practices among the labs & $x$ & & & & & & & \\
\hline \multicolumn{9}{|l|}{ to build a cooperative network } \\
\hline \multicolumn{9}{|l|}{ of government, academic and } \\
\hline \multicolumn{9}{|l|}{ research institutions for the } \\
\hline \multicolumn{9}{|l|}{ purpose of developing the } \\
\hline \multicolumn{9}{|l|}{ countries digital ecosystem } \\
\hline \multicolumn{9}{|l|}{ jointly manage business } \\
\hline incubation process. & $x$ & & & & & & & \\
\hline building a deeper interaction & $x$ & & & & & & & \\
\hline
\end{tabular}




\begin{tabular}{|c|c|c|c|c|c|c|c|c|}
\hline \multirow{2}{*}{ Phrase } & \multirow[b]{2}{*}{ Collaboration } & \multirow{2}{*}{$\begin{array}{l}\text { Social } \\
\text { Impact }\end{array}$} & \multirow{2}{*}{$\begin{array}{c}\text { Business } \\
\text { Development }\end{array}$} & \multirow{2}{*}{$\begin{array}{c}\text { Economic } \\
\text { Development }\end{array}$} & \multirow{2}{*}{$\begin{array}{l}\text { User } \\
\text { Impact }\end{array}$} & \multirow[b]{2}{*}{ ICT } & \multirow{2}{*}{$\begin{array}{l}\text { Test } \\
\text { Bed }\end{array}$} & \multirow[b]{2}{*}{ Framework } \\
\hline & & & & & & & & \\
\hline \multicolumn{9}{|l|}{ with technology entrepreneurs } \\
\hline \multicolumn{9}{|l|}{ and venture investors to build } \\
\hline \multicolumn{9}{|l|}{ viable and innovative business. } \\
\hline \multicolumn{9}{|l|}{ exploit the common daily lives } \\
\hline \multicolumn{9}{|l|}{ of its citizens and their digital } \\
\hline habits to help innovate & $\mathrm{x}$ & & & & & & & \\
\hline \multicolumn{9}{|l|}{ to help facilitate open- } \\
\hline \multicolumn{9}{|l|}{ innovation, citizen } \\
\hline \multicolumn{9}{|l|}{ participation in co-creation } \\
\hline activities & $x$ & & & & & & & \\
\hline \multicolumn{9}{|l|}{ exploration of new business } \\
\hline \multicolumn{9}{|l|}{ models for IP management, } \\
\hline \multicolumn{9}{|l|}{ licensing agreement and joint } \\
\hline venture for market expansion. & $x$ & & & & & & & \\
\hline \multicolumn{9}{|l|}{ advocates for the creation of a } \\
\hline \multicolumn{9}{|l|}{ community of knowledge in } \\
\hline sport. & $x$ & & & & & & & \\
\hline \multicolumn{9}{|l|}{ promotes the creation and } \\
\hline \multicolumn{9}{|l|}{ sustaining of partnerships and } \\
\hline \multicolumn{9}{|l|}{ relationships, promotes } \\
\hline \multicolumn{9}{|l|}{ collaboration on different } \\
\hline \multicolumn{9}{|l|}{ projects, and access to } \\
\hline \multicolumn{9}{|l|}{ information and know-how } \\
\hline knowledge. & $x$ & & & & & & & \\
\hline To jointly manage the & & & & & & & & \\
\hline technology incubation process & $\mathrm{x}$ & & & & & & & \\
\hline an ecosystem of living labs (in & & & & & & & & \\
\hline the city) and their associated & & & & & & & & \\
\hline innovation projects. & $x$ & & & & & & & \\
\hline to harmonize together the & & & & & & & & \\
\hline living labs of the city & $x$ & & & & & & & \\
\hline raising the level of innovation & $x$ & & & & & & & \\
\hline
\end{tabular}




\begin{tabular}{|c|c|c|c|c|c|c|c|c|}
\hline & & Social & Business & Economic & User & & Test & \\
\hline Phrase & Collaboration & Impact & Development & Development & Impact & ICT & Bed & Framework \\
\hline \multicolumn{9}{|l|}{ and competitiveness in the } \\
\hline \multicolumn{9}{|l|}{ region. } \\
\hline \multicolumn{9}{|l|}{ and support a user-driven } \\
\hline \multicolumn{9}{|l|}{ innovation processes using } \\
\hline \multicolumn{9}{|l|}{ open and sustainable economic } \\
\hline methodologies, & $x$ & & & & & & & \\
\hline \multicolumn{9}{|l|}{ an ecosystem of end-users, } \\
\hline \multicolumn{9}{|l|}{ technology and specialists } \\
\hline \multicolumn{9}{|l|}{ working together to work on a } \\
\hline \multicolumn{9}{|l|}{ common goal of boosting ICT } \\
\hline innovation. & $x$ & & & & & & & \\
\hline \multicolumn{9}{|l|}{ to improve innovation capacity } \\
\hline \multicolumn{9}{|l|}{ by acquiring expertise of } \\
\hline \multicolumn{9}{|l|}{ biomedical professions, } \\
\hline caregivers and users & $x$ & & & & & & & \\
\hline \multicolumn{9}{|l|}{ to maintain stakeholder's } \\
\hline satisfaction & $x$ & & & & & & & \\
\hline \multicolumn{9}{|l|}{ to develop a creative, } \\
\hline \multicolumn{9}{|l|}{ supportive and motivating } \\
\hline \multicolumn{9}{|l|}{ network for its citizens and } \\
\hline \multicolumn{9}{|l|}{ towns that are tackling health } \\
\hline inequalities & $x$ & & & & & & & \\
\hline \multicolumn{9}{|l|}{ stimulating the innovation } \\
\hline production. & $x$ & & & & & & & \\
\hline \multicolumn{9}{|l|}{ to harness ICT as a tool for } \\
\hline open innovation, & $x$ & & & & & & & \\
\hline \multicolumn{9}{|l|}{ to establish a link between } \\
\hline \multicolumn{9}{|l|}{ municipality, business } \\
\hline \multicolumn{9}{|l|}{ enterprises, academia, media } \\
\hline and citizens & $x$ & & & & & & & \\
\hline \multicolumn{9}{|l|}{ to successfully collaborate } \\
\hline towards higher standards of & $x$ & & & & & & & \\
\hline
\end{tabular}




\begin{tabular}{|c|c|c|c|c|c|c|c|c|}
\hline \multirow{2}{*}{ Phrase } & \multirow[b]{2}{*}{ Collaboration } & \multirow{2}{*}{$\begin{array}{l}\text { Social } \\
\text { Impact }\end{array}$} & \multirow{2}{*}{$\begin{array}{c}\text { Business } \\
\text { Development }\end{array}$} & \multirow{2}{*}{$\begin{array}{c}\text { Economic } \\
\text { Development }\end{array}$} & \multirow{2}{*}{$\begin{array}{l}\text { User } \\
\text { Impact }\end{array}$} & \multirow[b]{2}{*}{ ICT } & \multirow{2}{*}{$\begin{array}{l}\text { Test } \\
\text { Bed }\end{array}$} & \multirow[b]{2}{*}{ Framework } \\
\hline & & & & & & & & \\
\hline \multicolumn{9}{|l|}{ society. } \\
\hline \multicolumn{9}{|l|}{ enable idea generation, start-up } \\
\hline entrepreneurship & $x$ & & & & & & & \\
\hline \multicolumn{9}{|l|}{ to organize territorial players } \\
\hline \multicolumn{9}{|l|}{ into a manner to adapt to the } \\
\hline \multicolumn{9}{|l|}{ evolution of the globalization } \\
\hline economy. & $x$ & & & & & & & \\
\hline \multicolumn{9}{|l|}{ to develop a discovery/training } \\
\hline \multicolumn{9}{|l|}{ space where ideas can be } \\
\hline \multicolumn{9}{|l|}{ picked up by companies and } \\
\hline \multicolumn{9}{|l|}{ turned into reality for further } \\
\hline innovation and growth. & $x$ & & & & & & & \\
\hline \multicolumn{9}{|l|}{ created a collaboration to } \\
\hline \multicolumn{9}{|l|}{ analyze the views and needs of } \\
\hline \multicolumn{9}{|l|}{ the target group in relation to } \\
\hline \multicolumn{9}{|l|}{ the real-life interaction of } \\
\hline \multicolumn{9}{|l|}{ information systems and } \\
\hline healthcare. & $x$ & & & & & & & \\
\hline \multicolumn{9}{|l|}{ to promote and accelerate the } \\
\hline \multicolumn{9}{|l|}{ production of innovative } \\
\hline information, & $x$ & & & & & & & \\
\hline \multicolumn{9}{|l|}{ The lab was created to facilitate } \\
\hline \multicolumn{9}{|l|}{ the collaborative work between } \\
\hline \multicolumn{9}{|l|}{ all actors, not just companies } \\
\hline and clients. & $x$ & & & & & & & \\
\hline reducing the transaction costs & & & & & & & & \\
\hline encountered for collaboration & & & & & & & & \\
\hline and setting joint operating rul & & & & & & & & \\
\hline for participants. & $x$ & & & & & & & \\
\hline provides a platform for & & & & & & & & \\
\hline business, research centers and & & & & & & & & \\
\hline government to come together & $x$ & & & & & & & \\
\hline
\end{tabular}




\begin{tabular}{|c|c|c|c|c|c|c|c|c|}
\hline Phrase & Collaboration & $\begin{array}{l}\text { Social } \\
\text { Impact }\end{array}$ & $\begin{array}{c}\text { Business } \\
\text { Development }\end{array}$ & $\begin{array}{c}\text { Economic } \\
\text { Development }\end{array}$ & $\begin{array}{l}\text { User } \\
\text { Impact }\end{array}$ & ICT & $\begin{array}{l}\text { Test } \\
\text { Bed }\end{array}$ & Framework \\
\hline \multicolumn{9}{|c|}{ and collaborate on "real world" } \\
\hline \multicolumn{9}{|l|}{ problems and help build } \\
\hline \multicolumn{9}{|l|}{ innovative solutions for the } \\
\hline \multicolumn{9}{|l|}{ future. } \\
\hline \multicolumn{9}{|l|}{ to bridge the groups into an } \\
\hline \multicolumn{9}{|l|}{ easy and effective } \\
\hline \multicolumn{9}{|l|}{ communication dynamic to } \\
\hline \multicolumn{9}{|l|}{ facilitate innovation } \\
\hline collaboration. & $x$ & & & & & & & \\
\hline to build a social network. & $\mathrm{x}$ & & & & & & & \\
\hline \multicolumn{9}{|l|}{ providing its actors various } \\
\hline \multicolumn{9}{|l|}{ developments, to their STP to } \\
\hline \multicolumn{9}{|c|}{ design, for development and } \\
\hline \multicolumn{9}{|c|}{ testing of their products in real } \\
\hline environments. & $x$ & & & & & & & \\
\hline to strengthen the industrial & & & & & & & & \\
\hline \multicolumn{9}{|l|}{ research and technology } \\
\hline transfer network, & $x$ & & & & & & & \\
\hline \multicolumn{9}{|l|}{ to enable co-creation where } \\
\hline \multicolumn{9}{|l|}{ everyone can develop his or } \\
\hline \multicolumn{9}{|l|}{ her projects without } \\
\hline limitations. & $x$ & & & & & & & \\
\hline \multicolumn{9}{|c|}{ close interaction with } \\
\hline \multicolumn{9}{|c|}{ researchers, policy makes and } \\
\hline \multicolumn{9}{|c|}{ business communities in order } \\
\hline \multicolumn{9}{|l|}{ to enhance the innovation } \\
\hline process. & $x$ & & \\
\hline \multicolumn{9}{|c|}{ encourages the adoption of an } \\
\hline inclusive habituate & & $x$ & & & & & & \\
\hline \multicolumn{9}{|l|}{ to insure a balance is } \\
\hline \multicolumn{9}{|l|}{ maintained between human } \\
\hline activity and nature & & $x$ & & & & & & \\
\hline
\end{tabular}




\begin{tabular}{|c|c|c|c|c|c|c|c|c|}
\hline & & Social & Business & Economic & User & & Test & \\
\hline Phrase & Collaboration & Impact & Development & Development & Impact & ICT & Bed & Framework \\
\hline \multicolumn{9}{|l|}{ preservation. } \\
\hline \multicolumn{9}{|l|}{ optimization of resources } \\
\hline \multicolumn{9}{|l|}{ regarding transport traffic in } \\
\hline urban areas. & & $x$ & & & & & & \\
\hline \multicolumn{9}{|l|}{ build a network for well-being } \\
\hline \multicolumn{9}{|l|}{ and further the Social } \\
\hline \multicolumn{9}{|l|}{ innovation and Social Inclusion } \\
\hline of participants. & & $x$ & & & & & & \\
\hline \multicolumn{9}{|l|}{ to have a more resourceful and } \\
\hline territory & & $x$ & & & & & & \\
\hline \multicolumn{9}{|l|}{ aimed at promoting the regions } \\
\hline innovation capacity. & & $x$ & & & & & & \\
\hline for rural development & & $x$ & & & & & & \\
\hline \multicolumn{9}{|l|}{ raising life quality and } \\
\hline solidarity of their community. & & $x$ & & & & & & \\
\hline \multicolumn{9}{|l|}{ to build up the regions } \\
\hline \multicolumn{9}{|l|}{ networks, services and data } \\
\hline infrastructures & & $x$ & & & & & & \\
\hline \multicolumn{9}{|l|}{ helping in the management and } \\
\hline \multicolumn{9}{|l|}{ the preservation of the } \\
\hline territory. & & $x$ & & & & & & \\
\hline \multicolumn{9}{|l|}{ to create model for future } \\
\hline \multicolumn{9}{|l|}{ sustainability of the societies } \\
\hline welfare for the region. & & $x$ & & & & & & \\
\hline \multicolumn{9}{|l|}{ to expand the social, } \\
\hline \multicolumn{9}{|l|}{ organizational and } \\
\hline \multicolumn{9}{|l|}{ technological dimensions of } \\
\hline innovation. & & $x$ & & & & & & \\
\hline \multicolumn{9}{|l|}{ of supporting personal and } \\
\hline \multicolumn{9}{|l|}{ professional women's growth } \\
\hline \multicolumn{9}{|l|}{ through knowledge and } \\
\hline innovation. & & $x$ & & & & & & \\
\hline
\end{tabular}




\begin{tabular}{|c|c|c|c|c|c|c|c|c|}
\hline Phrase & Collaboration & $\begin{array}{l}\text { Social } \\
\text { Impact }\end{array}$ & $\begin{array}{c}\text { Business } \\
\text { Development }\end{array}$ & $\begin{array}{c}\text { Economic } \\
\text { Development }\end{array}$ & $\begin{array}{l}\text { User } \\
\text { Impact }\end{array}$ & ICT & $\begin{array}{l}\text { Test } \\
\text { Bed }\end{array}$ & Framework \\
\hline \multicolumn{9}{|l|}{ to foster women's growth, } \\
\hline \multicolumn{9}{|l|}{ provide motivation and set an } \\
\hline \multicolumn{9}{|l|}{ open environment for } \\
\hline \multicolumn{9}{|l|}{ entrepreneurship so that they } \\
\hline \multicolumn{9}{|l|}{ may achieve their goals, realize } \\
\hline \multicolumn{9}{|l|}{ their role in society and } \\
\hline \multicolumn{9}{|l|}{ contribute to the territories } \\
\hline economy. & & $x$ & & & & & & \\
\hline \multicolumn{9}{|l|}{ to help with finances, } \\
\hline \multicolumn{9}{|l|}{ education and mentoring of the } \\
\hline \multicolumn{9}{|l|}{ women in topics of health, } \\
\hline \multicolumn{9}{|l|}{ personal growth, taxes, } \\
\hline legalities and exports. & & $\mathrm{x}$ & & & & & & \\
\hline an ecosystem and social & & & & & & & & \\
\hline \multicolumn{9}{|l|}{ network for the Mexican } \\
\hline \multicolumn{9}{|l|}{ women where they can access } \\
\hline \multicolumn{9}{|l|}{ and contribute tacit knowledge } \\
\hline and support. & & $x$ & & & & & & \\
\hline \multicolumn{9}{|l|}{ to improve traffic and driving } \\
\hline \multicolumn{9}{|l|}{ to reduce accidents and traffic } \\
\hline \multicolumn{2}{|l|}{ jams. } & $x$ & & & & & & \\
\hline \multicolumn{9}{|l|}{ for socio-economic } \\
\hline \multicolumn{9}{|l|}{ development and positively } \\
\hline \multicolumn{9}{|l|}{ impact the groups of disabled } \\
\hline people & & $\mathrm{x}$ & & & & & & \\
\hline \multicolumn{9}{|l|}{ improving social and cultural } \\
\hline \multicolumn{9}{|l|}{ life in the region through their } \\
\hline \multicolumn{9}{|l|}{ output in social innovations, } \\
\hline \multicolumn{9}{|l|}{ social enterprise spinouts and } \\
\hline the building of social networks. & & $x$ & & & & & & \\
\hline \multicolumn{9}{|l|}{ to grow the digital inclusion } \\
\hline paradigm that plays a role in & & $x$ & & & & & & \\
\hline
\end{tabular}




\begin{tabular}{|c|c|c|c|c|c|c|c|c|}
\hline Phrase & Collaboration & $\begin{array}{l}\text { Social } \\
\text { Impact }\end{array}$ & $\begin{array}{c}\text { Business } \\
\text { Development }\end{array}$ & $\begin{array}{c}\text { Economic } \\
\text { Development }\end{array}$ & $\begin{array}{l}\text { User } \\
\text { Impact }\end{array}$ & ICT & $\begin{array}{l}\text { Test } \\
\text { Bed }\end{array}$ & Framework \\
\hline \multicolumn{9}{|l|}{ social inclusion; } \\
\hline \multicolumn{9}{|l|}{ to help increase digital literacy } \\
\hline \multicolumn{9}{|l|}{ platforms or employment } \\
\hline portals to its citizens. & & $\mathrm{x}$ & & & & & & \\
\hline \multicolumn{9}{|l|}{ to positively transform the } \\
\hline \multicolumn{9}{|l|}{ country away from violence, } \\
\hline \multicolumn{9}{|l|}{ corruption and other disasters, } \\
\hline \multicolumn{9}{|l|}{ to a more prosperous social and } \\
\hline economic state. & & $x$ & & & & & & \\
\hline \multicolumn{9}{|l|}{ Through social innovation, } \\
\hline \multicolumn{9}{|l|}{ social inclusion, tourism, } \\
\hline \multicolumn{9}{|l|}{ cultural services, development } \\
\hline \multicolumn{9}{|l|}{ of smart region and security, } \\
\hline \multicolumn{9}{|l|}{ will improve lives of its } \\
\hline \multicolumn{9}{|l|}{ citizens and become a pilot } \\
\hline \multicolumn{9}{|l|}{ model for other developing } \\
\hline countries. & & $\mathrm{x}$ & & & & & & \\
\hline \multicolumn{9}{|l|}{ to assist the elderly and } \\
\hline \multicolumn{9}{|l|}{ physically/cognitively- } \\
\hline impaired individuals. & & $x$ & & & & & & \\
\hline \multicolumn{9}{|l|}{ raising citizen education and } \\
\hline control in there area. & & $\mathrm{x}$ & & & & & & \\
\hline \multicolumn{9}{|l|}{ help companies, NGO's and } \\
\hline \multicolumn{9}{|l|}{ government organizations to } \\
\hline \multicolumn{9}{|l|}{ design build and test products } \\
\hline \multicolumn{9}{|l|}{ for improving lives of older } \\
\hline people. & & $\mathrm{x}$ & & & & & & \\
\hline \multicolumn{9}{|l|}{ focuses on the needs of its } \\
\hline \multicolumn{9}{|l|}{ citizens and the development of } \\
\hline its territory. & & $x$ & & & & & & \\
\hline To lower their carbon impact & & $x$ & & & & & & \\
\hline
\end{tabular}




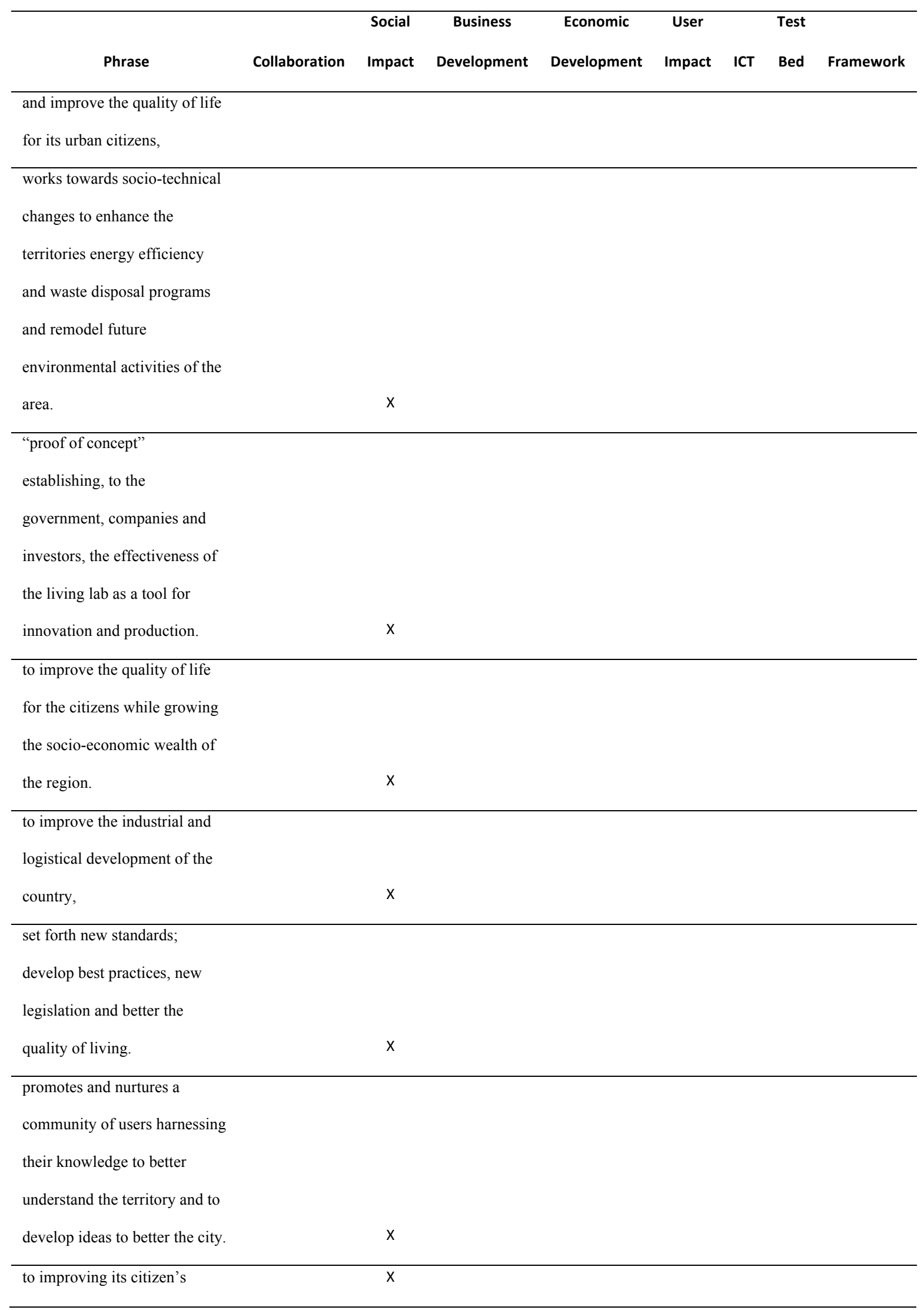




\begin{tabular}{|c|c|c|c|c|c|c|c|c|}
\hline & & Social & Business & Economic & User & & Test & \\
\hline Phrase & Collaboration & Impact & Development & Development & Impact & ICT & Bed & Framework \\
\hline \multicolumn{9}{|l|}{ lifestyles through solutions } \\
\hline \multicolumn{9}{|l|}{ targeting their social needs. } \\
\hline \multicolumn{9}{|l|}{ to build a better touristic, and } \\
\hline cultural heritage for the region & & $x$ & & & & & & \\
\hline \multicolumn{9}{|l|}{ turning an old and degraded } \\
\hline \multicolumn{9}{|l|}{ industrial area into a space } \\
\hline \multicolumn{9}{|l|}{ where participants can freely } \\
\hline \multicolumn{9}{|l|}{ express themselves and share } \\
\hline experiences & & $x$ & & & & & & \\
\hline \multicolumn{9}{|l|}{ bring together the local actors } \\
\hline \multicolumn{9}{|l|}{ to promote the continued } \\
\hline \multicolumn{9}{|l|}{ development of Smart City } \\
\hline technology. & & $x$ & & & & & & \\
\hline focused on the economy of & & & & & & & & \\
\hline knowledge & & & $x$ & & & & & \\
\hline \multicolumn{9}{|l|}{ to establish a Knowledge } \\
\hline Society Ecosystem (KSEco) & & & $x$ & & & & & \\
\hline \multicolumn{9}{|l|}{ a platform for MSMEs to } \\
\hline \multicolumn{9}{|l|}{ collect knowledge for specific } \\
\hline purposes. & & & $x$ & & & & & \\
\hline \multicolumn{9}{|l|}{ to achieve working prototypes } \\
\hline \multicolumn{9}{|l|}{ of innovation to showcase the } \\
\hline \multicolumn{9}{|l|}{ potential of the living lab and } \\
\hline \multicolumn{9}{|l|}{ allow for measurements of } \\
\hline performance. & & & $x$ & & & & & \\
\hline \multicolumn{9}{|l|}{ a means to further develop, } \\
\hline \multicolumn{9}{|l|}{ demonstrate and showcase } \\
\hline \multicolumn{9}{|l|}{ scenarios for future techniques } \\
\hline \multicolumn{9}{|l|}{ in teaching and learning as a } \\
\hline \multicolumn{9}{|l|}{ result of the role technology } \\
\hline plays in today's society. & & & $x$ & & & & & \\
\hline gives policy makers an & & & $x$ & & & & & \\
\hline
\end{tabular}




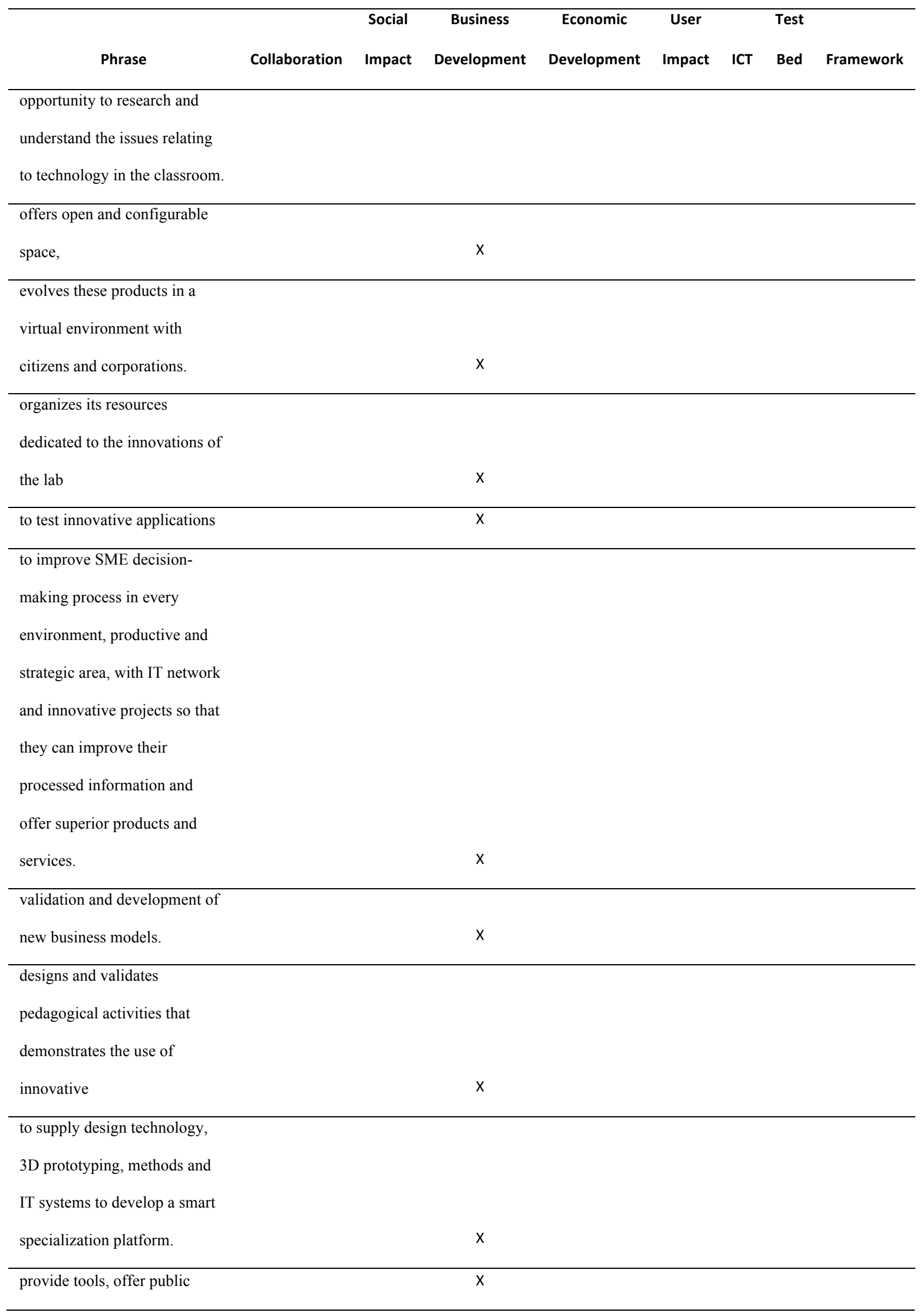




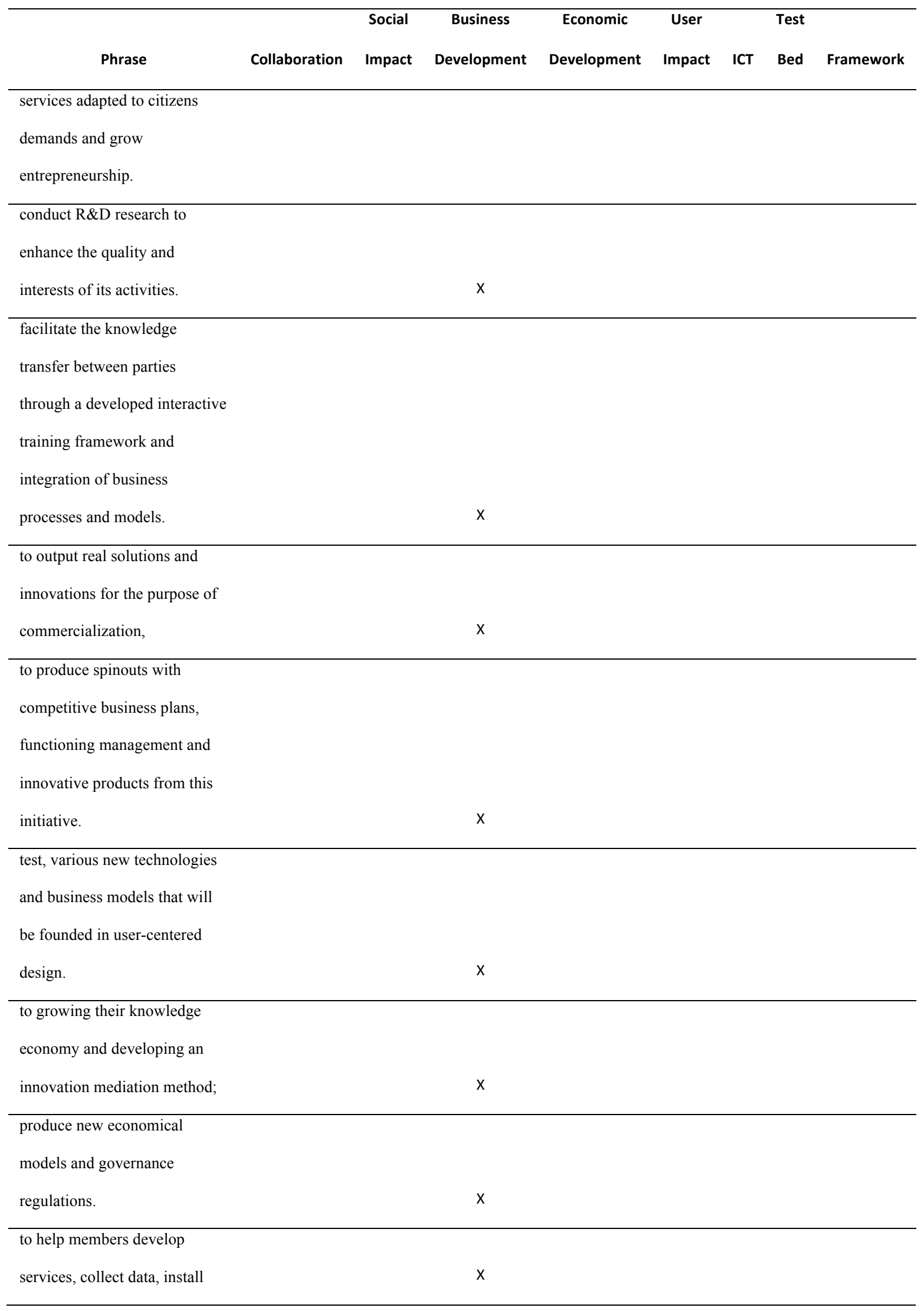




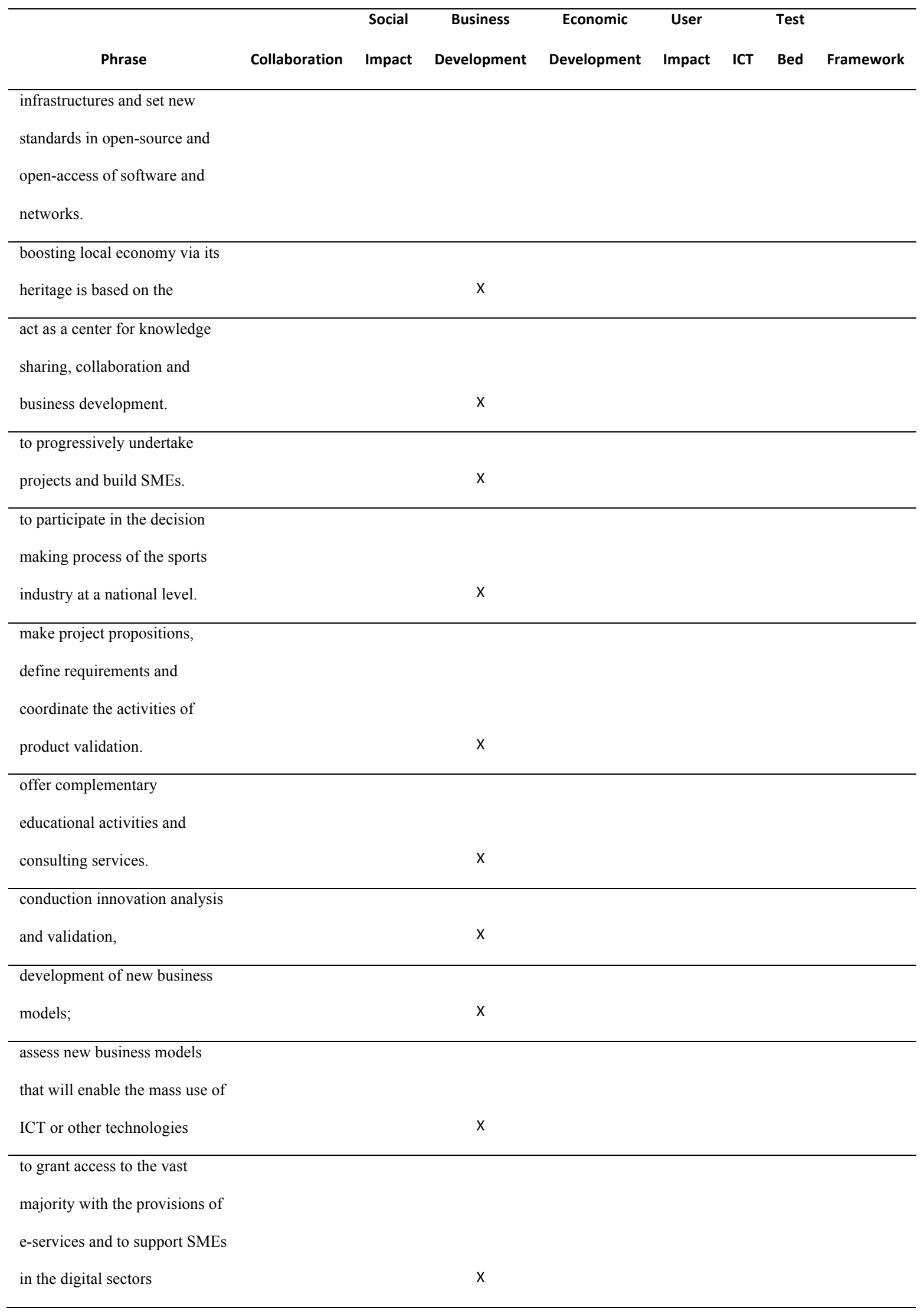




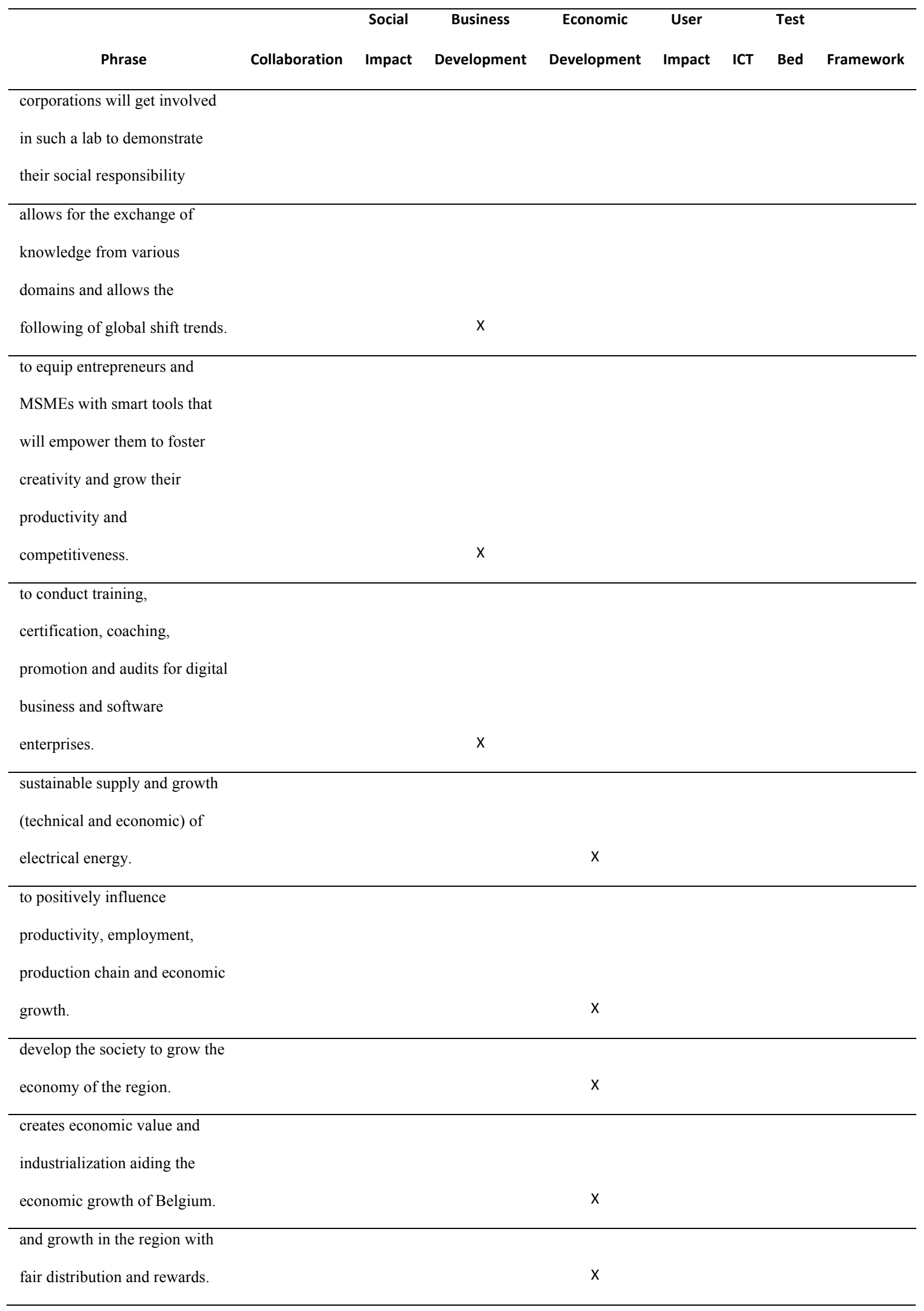




\begin{tabular}{|c|c|c|c|c|c|c|c|c|}
\hline & & Social & Business & Economic & User & & Test & \\
\hline Phrase & Collaboration & Impact & Development & Development & Impact & ICT & Bed & Framework \\
\hline to build a better, economic & & & & $\mathrm{x}$ & & & & \\
\hline \multicolumn{9}{|l|}{ boosting creative economy and } \\
\hline cultural heritage. & & & & $x$ & & & & \\
\hline \multicolumn{9}{|l|}{ a response to the changing } \\
\hline \multicolumn{9}{|l|}{ climate of technologies and the } \\
\hline \multicolumn{9}{|l|}{ need to update the production } \\
\hline \multicolumn{9}{|l|}{ districts so that they may } \\
\hline \multicolumn{9}{|l|}{ continue to play an important } \\
\hline \multicolumn{9}{|l|}{ role in the community and } \\
\hline maintain the economy. & & & & $x$ & & & & \\
\hline \multicolumn{9}{|l|}{ to grow new business and } \\
\hline \multicolumn{9}{|l|}{ strengthen entrepreneurship } \\
\hline throughout the country. & & & & $x$ & & & & \\
\hline \multicolumn{9}{|l|}{ is to attract business } \\
\hline investments into the regions & & & & $x$ & & & & \\
\hline \multicolumn{9}{|l|}{ build up from existing or } \\
\hline \multicolumn{9}{|l|}{ scratch the economic sector of } \\
\hline the territory. & & & & $x$ & & & & \\
\hline to accelerate the growth of & & & & & & & & \\
\hline business and entrepreneurship. & & & & $x$ & & & & \\
\hline \multicolumn{9}{|l|}{ to enhance the economy of the } \\
\hline region and abroad. & & & & $x$ & & & & \\
\hline \multicolumn{9}{|l|}{ to promote economic and } \\
\hline \multicolumn{9}{|l|}{ social regeneration through an } \\
\hline \multicolumn{9}{|l|}{ artistic, innovative and creative } \\
\hline urban transformation & & & & $x$ & & & & \\
\hline attract public to the region. & & & & $\mathrm{x}$ & & & & \\
\hline \multicolumn{9}{|l|}{ to fuel the economic activity in } \\
\hline the region, & & & & $\mathrm{x}$ & & & & \\
\hline \multicolumn{9}{|l|}{ fostering economic growth and } \\
\hline competitiveness. & & & & $x$ & & & & \\
\hline optimizing life of care- & & & & & $x$ & & & \\
\hline
\end{tabular}




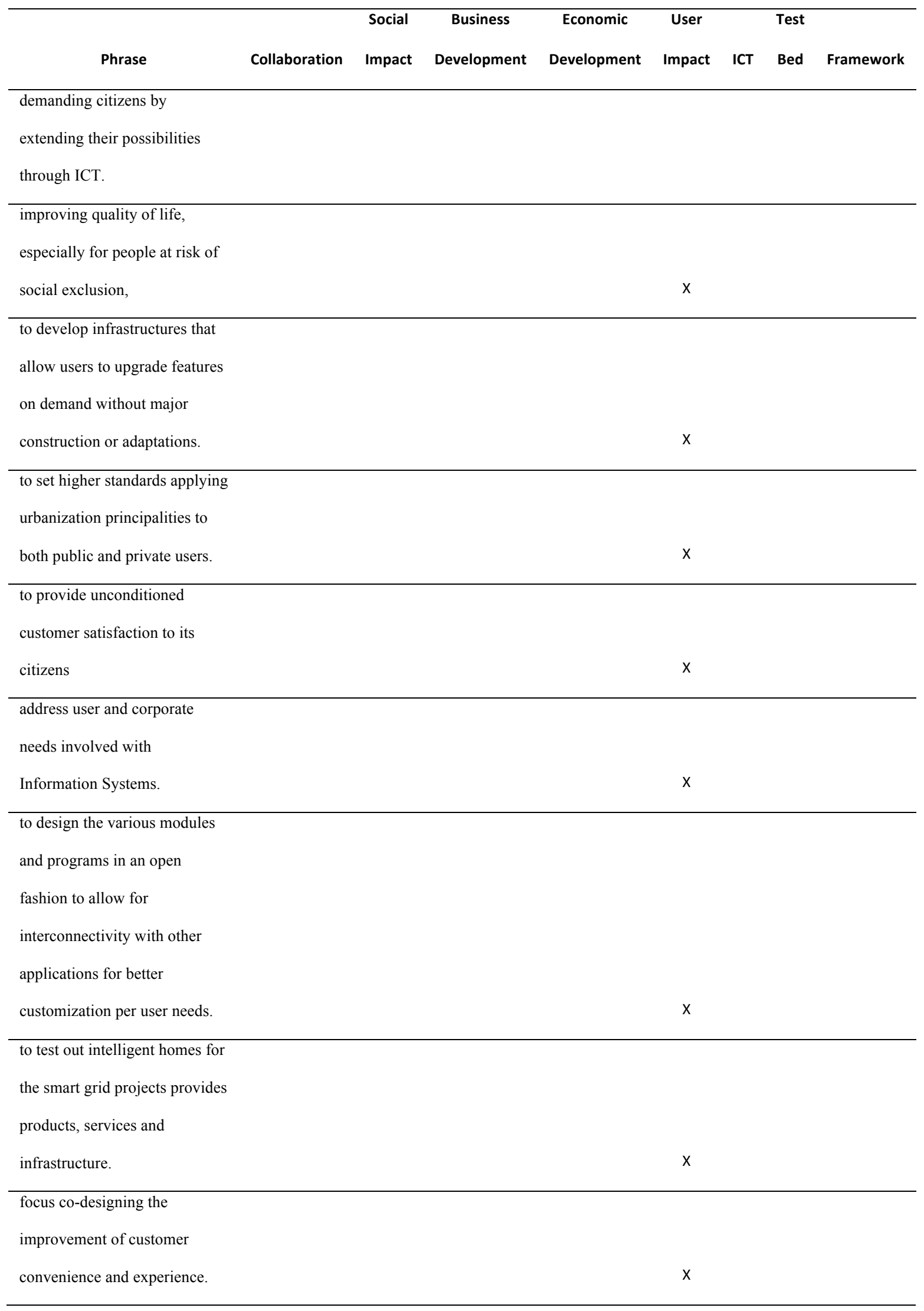




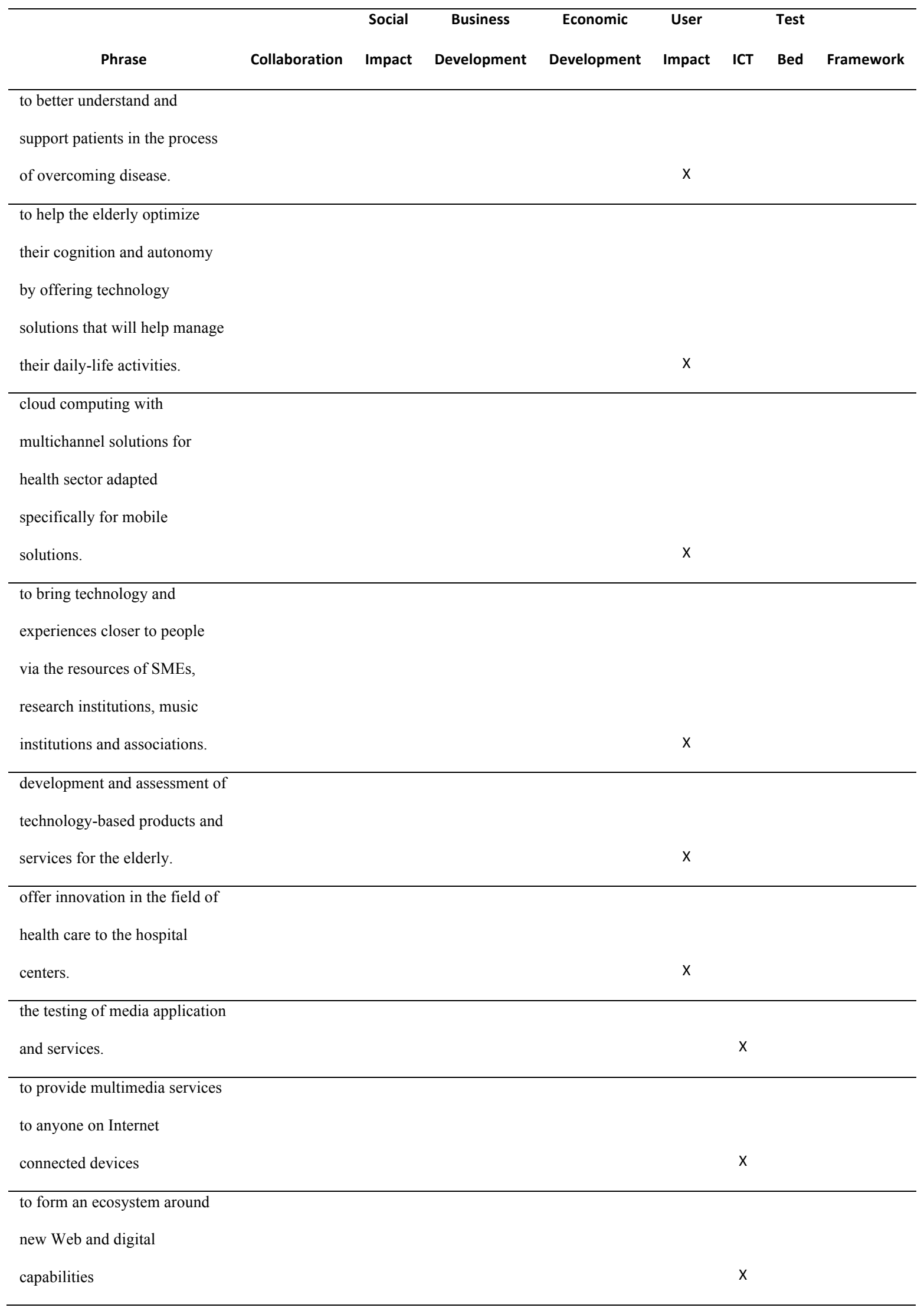




\begin{tabular}{|c|c|c|c|c|c|c|c|c|}
\hline & & Social & Business & Economic & User & & Test & \\
\hline Phrase & Collaboration & Impact & Development & Development & Impact & ICT & Bed & Framework \\
\hline raise awareness towards ICT & & & & & & $x$ & & \\
\hline \multicolumn{9}{|l|}{ communication and } \\
\hline \multicolumn{9}{|l|}{ entertainment (ICE) products } \\
\hline and services. & & & & & & $x$ & & \\
\hline \multicolumn{9}{|l|}{ to utilize ICT and its } \\
\hline \multicolumn{9}{|l|}{ advantages in managing } \\
\hline \multicolumn{9}{|l|}{ information to achieve its } \\
\hline goals. & & & & & & $x$ & & \\
\hline \multicolumn{9}{|l|}{ developing and testing ICT } \\
\hline \multicolumn{9}{|l|}{ services for public } \\
\hline administrators & & & & & & $x$ & & \\
\hline \multicolumn{9}{|l|}{ to become the regional } \\
\hline governing body over ICT. & & & & & & $x$ & & \\
\hline \multicolumn{9}{|l|}{ an ecosystem of end-users, } \\
\hline \multicolumn{9}{|l|}{ technology and specialists } \\
\hline \multicolumn{9}{|l|}{ working together to work on a } \\
\hline \multicolumn{9}{|l|}{ common goal of boosting ICT } \\
\hline innovation. & & & & & & $x$ & & \\
\hline \multicolumn{9}{|l|}{ to research the use of ICT to } \\
\hline \multicolumn{9}{|l|}{ maintain the cultural heritage } \\
\hline \multicolumn{9}{|l|}{ and grow the arts, media and } \\
\hline functional music production. & & & & & & $x$ & & \\
\hline help promote the use of ICT & & & & & & $x$ & & \\
\hline \multicolumn{9}{|l|}{ aims to bring next generation } \\
\hline broadband to the region. & & & & & & $x$ & & \\
\hline \multicolumn{9}{|l|}{ optimizing life of care- } \\
\hline \multicolumn{9}{|l|}{ demanding citizens by } \\
\hline \multicolumn{9}{|l|}{ extending their possibilities } \\
\hline through ICT. & & & & & & $x$ & & \\
\hline \multicolumn{9}{|l|}{ to encourage development for } \\
\hline \multicolumn{9}{|l|}{ fibre technology and lower the } \\
\hline entry barrier for "internet- & & & & & & $x$ & & \\
\hline
\end{tabular}




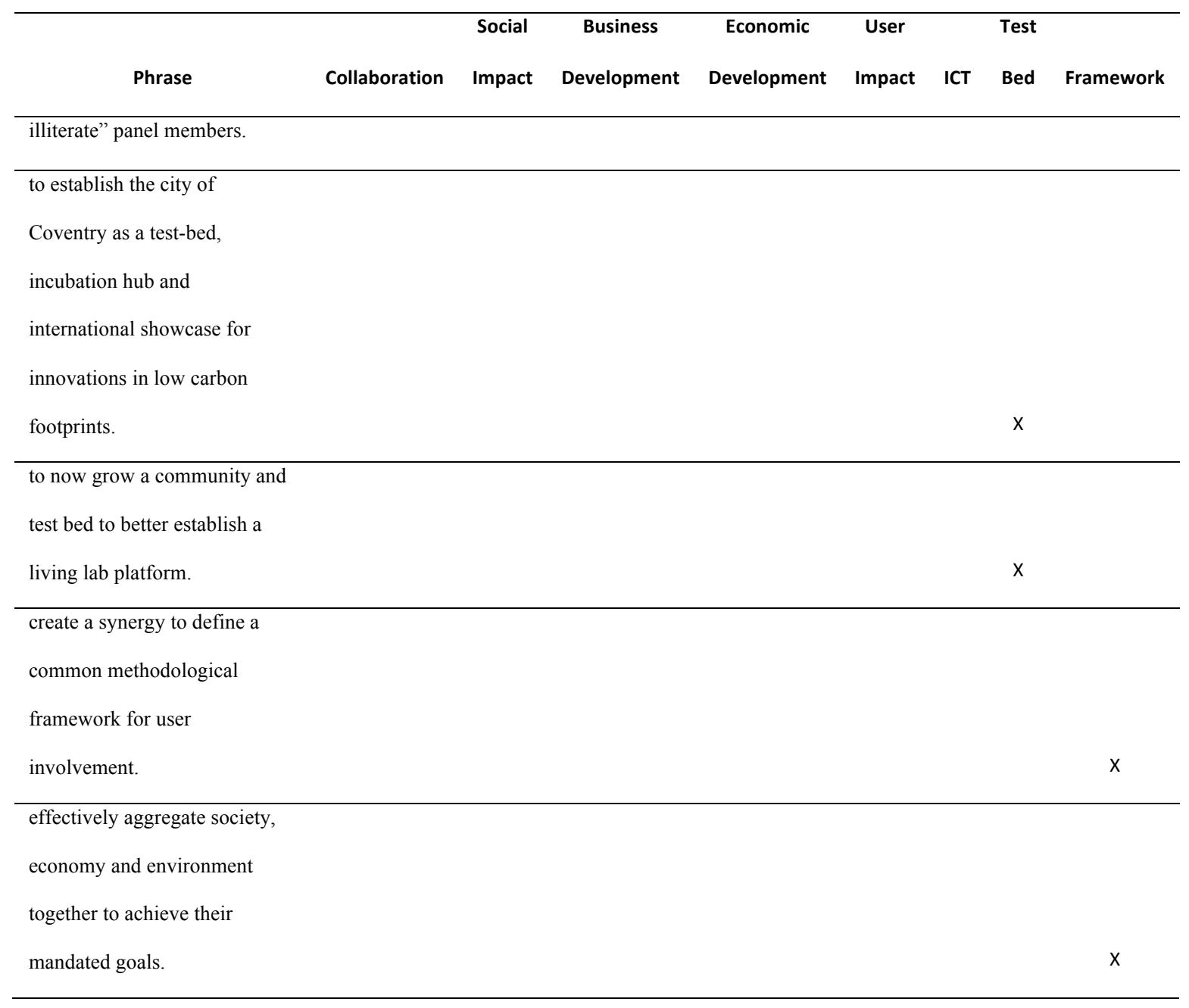


Table 10 IPR Management Data points Assorted into Respective Categories

\begin{tabular}{|c|c|c|c|c|c|c|c|}
\hline \multirow[b]{2}{*}{ Phrase } & \multirow[b]{2}{*}{ Agreement } & \multirow[b]{2}{*}{ OEM } & \multicolumn{5}{|c|}{ Case-by- } \\
\hline & & & License & Open & case & Law & Other \\
\hline \multicolumn{8}{|l|}{ Through specific agreements upholds social and } \\
\hline specific innovation between the members. & $\mathrm{X}$ & & & & & & \\
\hline \multicolumn{8}{|l|}{ forms joint ownership management agreements to } \\
\hline \multicolumn{8}{|l|}{ regulate the arrangements between participating } \\
\hline members & $\mathrm{X}$ & & & & & & \\
\hline \multicolumn{8}{|l|}{ joint agreements to regulates Intellectual Property } \\
\hline Policy. & $\mathrm{X}$ & & & & & & \\
\hline \multicolumn{8}{|l|}{ partner, grants free right of use of knowledge and } \\
\hline \multicolumn{8}{|l|}{ exploitation of the IP provided by framework of lab } \\
\hline \multicolumn{8}{|l|}{ that other members can participate in the labs } \\
\hline projects. Agreement & $\mathrm{X}$ & & & & & & \\
\hline \multicolumn{8}{|l|}{, the lab encourages to make it available creative } \\
\hline common terms. Agreement & $\mathrm{X}$ & & & & & & \\
\hline \multicolumn{8}{|l|}{ each project undergoes IPR reviews before } \\
\hline \multicolumn{8}{|l|}{ commencement and a formal agreement is drafted } \\
\hline and signed to ensure satisfactory IP control. & $\mathrm{X}$ & & & & & & \\
\hline \multicolumn{8}{|c|}{ IPR policy are outlined in the consortium agreement. } \\
\hline which all members must sign, & $\mathrm{X}$ & & & & & & \\
\hline \multicolumn{8}{|l|}{ not force any IPR regulations but recommends ideas } \\
\hline be protect by confidentiality agreements & $\mathrm{X}$ & & & & & & \\
\hline \multicolumn{8}{|l|}{ members have agreed to operate under the labs IPR } \\
\hline policy. & $\mathrm{X}$ & & & & & & \\
\hline \multicolumn{8}{|l|}{ IP and copyrighted material is kept confidential and } \\
\hline protected under the living lab rules. Agreement & $\mathrm{X}$ & & & & & & \\
\hline \multicolumn{8}{|l|}{ a consortium agreement that outlines IPR rules and } \\
\hline governing philosophies for all members & $\mathrm{X}$ & & & & & & \\
\hline \multicolumn{8}{|c|}{ agreement removes the fear of members attempting to } \\
\hline \multicolumn{8}{|l|}{ claim ownership of IPR without clear and mutual } \\
\hline upfront approval & $\mathrm{X}$ & & & & & & \\
\hline \multicolumn{8}{|c|}{ IPR is done through the consortium agreement that is } \\
\hline signed by all participating members. & $\mathrm{X}$ & & & & & & \\
\hline
\end{tabular}


Case-by-

Phrase Agreement OEM License Open case Law Other

a non-disclosure agreement in the consortium

agreement to control the sharing of information

during the set-up of the project, software protocols

and more.

$\mathrm{X}$

projects that have been initiated prior to their

involvement, it adopts the rules originally agreed

upon of that project.

$\mathrm{X}$

agreement outlines the IPR rules and confidentiality

of the innovations and results.

$\mathrm{X}$

Depending on the level of R\&D material and the

context within the project is being developed; and

the lab will act according to the Grant agreement

provided by its members.

$\mathrm{X}$

IPR in accordance with the agreements signed and industry standards.

X

All members sign consortium agreements which limit

the partners ability to use, license and share the rights

to the outputs

$\mathrm{X}$

each project independently and prepares IPR

agreements for each. $\quad \mathrm{X}$

joint IPs is controlled through the shared agreement. $\quad \mathrm{X}$

IPR principles through a signed consortium

agreement that point out the allowances and

limitations and knowledge sharing.

$\mathrm{X}$

to effectively divide cost between actors and reward

stakeholder contributions, sign partnership agreement

for licensing of product and services

$\mathrm{X}$

bases and upon agreement of the involved

participants.

$\mathrm{X}$

agreed to in application form.

$\mathrm{X}$

original equipment manufacturers still retain their

IPR

X 
Case-by-

Phrase Agreement OEM License Open case Law Other

the partner providing the idea or product retains the

IP.

$\mathrm{X}$

background IP ownership remains with provider,

orginal $\mathrm{X}$

IP of output be owned by its creator, orginal $\quad \mathrm{X}$

Each member is allowed to protect their idea, artistic

work, trademarkible symbols or phrases and

patenting their innovation. Orginal

$\mathrm{X}$

first approach is "Moral Rights", depending on the

projects context and associated regulation would be

the property of the producer. Orginal $\quad \mathrm{X}$

honors member's IPR. Orginal $\quad \mathrm{X}$

original IPs contributed by the industry members

remain the property of those members and shall be

exercised according to their will. Orginal $\quad \mathrm{X}$

\begin{tabular}{ll}
\hline private member owns the IP orginal & $\mathrm{X}$
\end{tabular}

company IP will be respected $\quad \mathrm{X}$

the respects members intellectual properties. $\mathrm{X}$

commercialization of products or services developed,

partner is unrestricted to negotiate the terms of use of

their own IP licenses.

$\mathrm{X}$

output innovations from collaboration are licensed

under fair and reasonable market conditions.

$\mathrm{X}$

"Essential IPR" is defined and made available under

license as

$\mathrm{X}$

gives permission to the business to commercialize it

and expand it into other markets. License

$\mathrm{X}$

lab would control the IP and license the technology to

a company for its commercialization, receiving

royalties from the innovation.

$\mathrm{X}$

Creative Commons BY-SA license for text, media

and other content produced in-house.

X 
Case-by-

Phrase Agreement OEM License Open case Law Other

participants provide each other with licenses for the

use and further development of the products.

$\mathrm{X}$

mutual access be made available to a high number of

commercial products and provide licensing of

development. License $\quad$ X

$\begin{array}{ll}\text { patent licenses } & \mathrm{X}\end{array}$

intellectual property governed by the open licenses

for each integrated open source module. $\quad \mathrm{X}$

\begin{tabular}{ll}
\hline uses open source licenses. & $\mathrm{X}$ \\
\hline
\end{tabular}

selective opening model to allocation, marketing,

modifying or distributing the product. $\quad \mathrm{X}$

Public funding makes IPR becomes open so that it

may be exploited. to enable openness and allows

society to benefit from the outcomes. $\quad \mathrm{X}$

\begin{tabular}{lc}
\hline all apps and outputs are open. & $\mathrm{X}$
\end{tabular}

Public content is governed under open-source

licenses such as GPL

$\mathrm{X}$

uses open and common creative licenses such as GPL

or the CC-BY-NC to manage their IPR.

$\mathrm{X}$

other licensing schemes, will adopt as needed to

maintain open source and open innovation

environment.

$\mathrm{X}$

applies GNU public licence for all the software

developed by their organization open

X

deal with ever intellectual property on an individual

bases considering the type of product, service and

member contribution.

X

intellectual property rights of each project will be

managed on case-by-case

X

The lab does not make or guarantee the licensing of

IPR, individual members must seek to resolve such

issues independently.

X 


\begin{tabular}{|c|c|c|c|c|c|c|}
\hline \multirow[b]{2}{*}{ Phrase } & \multirow[b]{2}{*}{ Agreement } & \multicolumn{5}{|c|}{ Case-by- } \\
\hline & & License & Open & case & Law & Other \\
\hline deals with each project individually for IP & & & & $\mathrm{X}$ & & \\
\hline \multicolumn{7}{|l|}{ IPR on a project by project defined by their } \\
\hline participating members. Case & & & & $\mathrm{X}$ & & \\
\hline \multicolumn{7}{|l|}{ not established any special IPR policies or } \\
\hline information control mechanisms case & & & & $\mathrm{X}$ & & \\
\hline IPR principals on a project-by-project case & & & & $\mathrm{X}$ & & \\
\hline \multicolumn{7}{|l|}{ deals with IPR issues on project-by-project bases. } \\
\hline Case & & & & $\mathrm{X}$ & & \\
\hline \multicolumn{7}{|c|}{ Intellectual Property Rights issues on a case-by-case } \\
\hline situation. & & & & $\mathrm{X}$ & & \\
\hline \multicolumn{7}{|c|}{ Following Colombian law, consistent with the World } \\
\hline Trade Organization, & & & & & $\mathrm{X}$ & \\
\hline IPR rules simply follows the countries set lav & & & & & $\mathrm{X}$ & \\
\hline \multicolumn{7}{|c|}{ innovation are patented in Mexico and United states } \\
\hline to protect the members rights. Law & & & & & $\mathrm{X}$ & \\
\hline \multicolumn{7}{|l|}{ follows Colombian IPR laws when handling of } \\
\hline shared IP. & & & & & $\mathrm{X}$ & \\
\hline IPR laws and regulations. & & & & & $\mathrm{X}$ & \\
\hline \multicolumn{7}{|l|}{ private and institutional sponsorships are covered } \\
\hline under specific IPR policies. Law & & & & & $\mathrm{X}$ & \\
\hline \multicolumn{7}{|c|}{ intellectual property of developed innovations and } \\
\hline assets belongs to the municipality. & & & & & & $\mathrm{X}$ \\
\hline \multicolumn{7}{|c|}{ main client and sponsor of the lab, the university sees } \\
\hline \multicolumn{7}{|l|}{ fit to manage the IPR of prototypes developed by } \\
\hline companies for the living lab. & & & & & & $\mathrm{X}$ \\
\hline \multicolumn{7}{|l|}{ members make some of their background IPR } \\
\hline \multicolumn{7}{|c|}{ available to the lab to further facilitate its build and } \\
\hline innovation. & & & & & & $\mathrm{X}$ \\
\hline \multicolumn{7}{|c|}{ Inform of "essential IPR" as soon as they become } \\
\hline \multicolumn{7}{|c|}{ aware that it is related to the prepared or published } \\
\hline specifications. & & & & & & $\mathrm{X}$ \\
\hline none are obligated to conduct and IP search. & & & & & & $\mathrm{X}$ \\
\hline
\end{tabular}




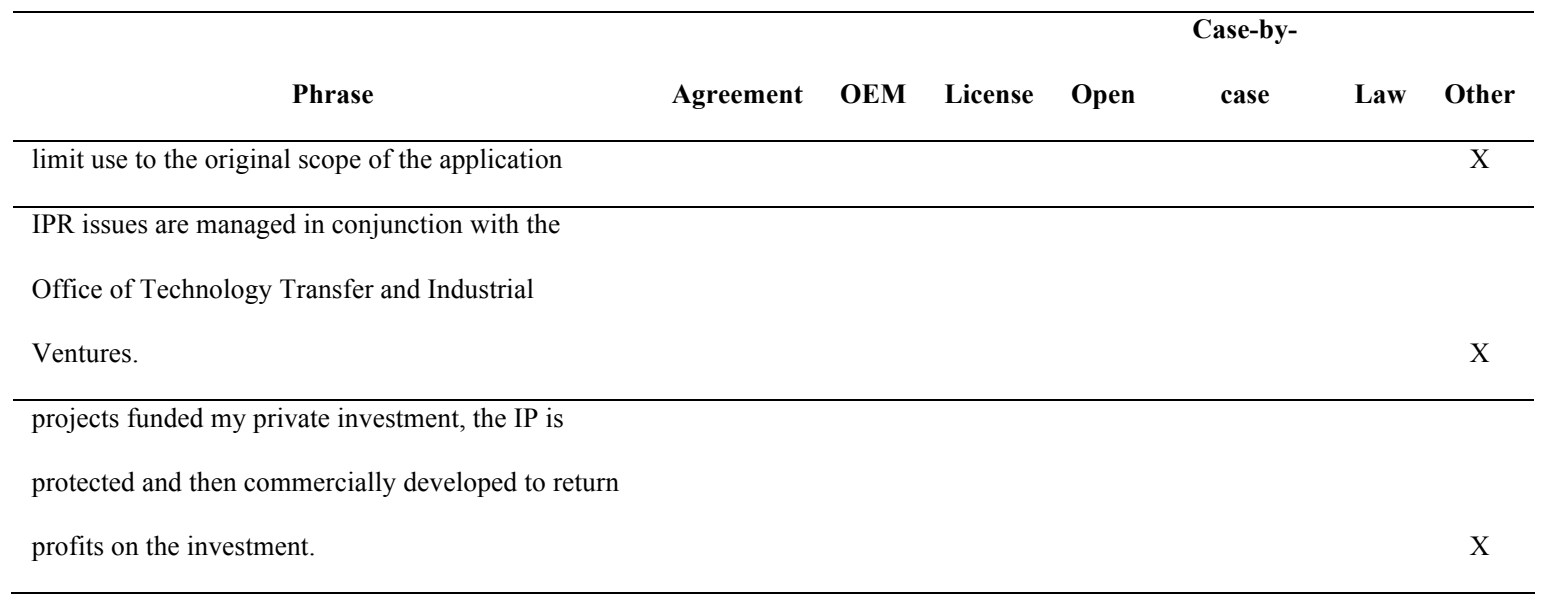

Table 11 Innovation Culture Data points Assorted into Respective Categories

\begin{tabular}{|c|c|c|c|c|c|c|}
\hline & Collective & Accepting & Open & & Open & \\
\hline Phrases & Effort & Members & Source & Agreement & thinking & Other \\
\hline believes in openness as a means to encourage collaboration & $\mathrm{X}$ & & & & & \\
\hline maintain ecosystem of collective intelligence. & $\mathrm{X}$ & & & & & \\
\hline \multicolumn{7}{|l|}{ all members must respect each other and work as a team as } \\
\hline an interdisciplinary group. & $\mathrm{X}$ & & & & & \\
\hline \multicolumn{7}{|l|}{ adopts an openness collaboration approach through its } \\
\hline \multicolumn{7}{|l|}{ cross-boarder initiatives and involvement of partner } \\
\hline organizations. & $\mathrm{X}$ & & & & & \\
\hline \multicolumn{7}{|l|}{ Open principles of teamwork, common good, cooperation } \\
\hline and community involvement & $\mathrm{X}$ & & & & & \\
\hline believes in social support and collaborative efforts. & $\mathrm{X}$ & & & & & \\
\hline \multicolumn{7}{|l|}{ fair and open collaboration providing unimpeded access to } \\
\hline any individual organization seeking to participate. & $\mathrm{X}$ & & & & & \\
\hline \multicolumn{7}{|l|}{ usage of open source technology a means to enable } \\
\hline collaborative & $\mathrm{X}$ & & & & & \\
\hline building and nurturing relations among members openness & $\mathrm{X}$ & & & & & \\
\hline \multicolumn{7}{|l|}{ cooperate towards scientific and entrepreneurial goals. } \\
\hline Collabo & $\mathrm{X}$ & & & & & \\
\hline openness and collaboration. & $\mathrm{X}$ & & & & & \\
\hline
\end{tabular}




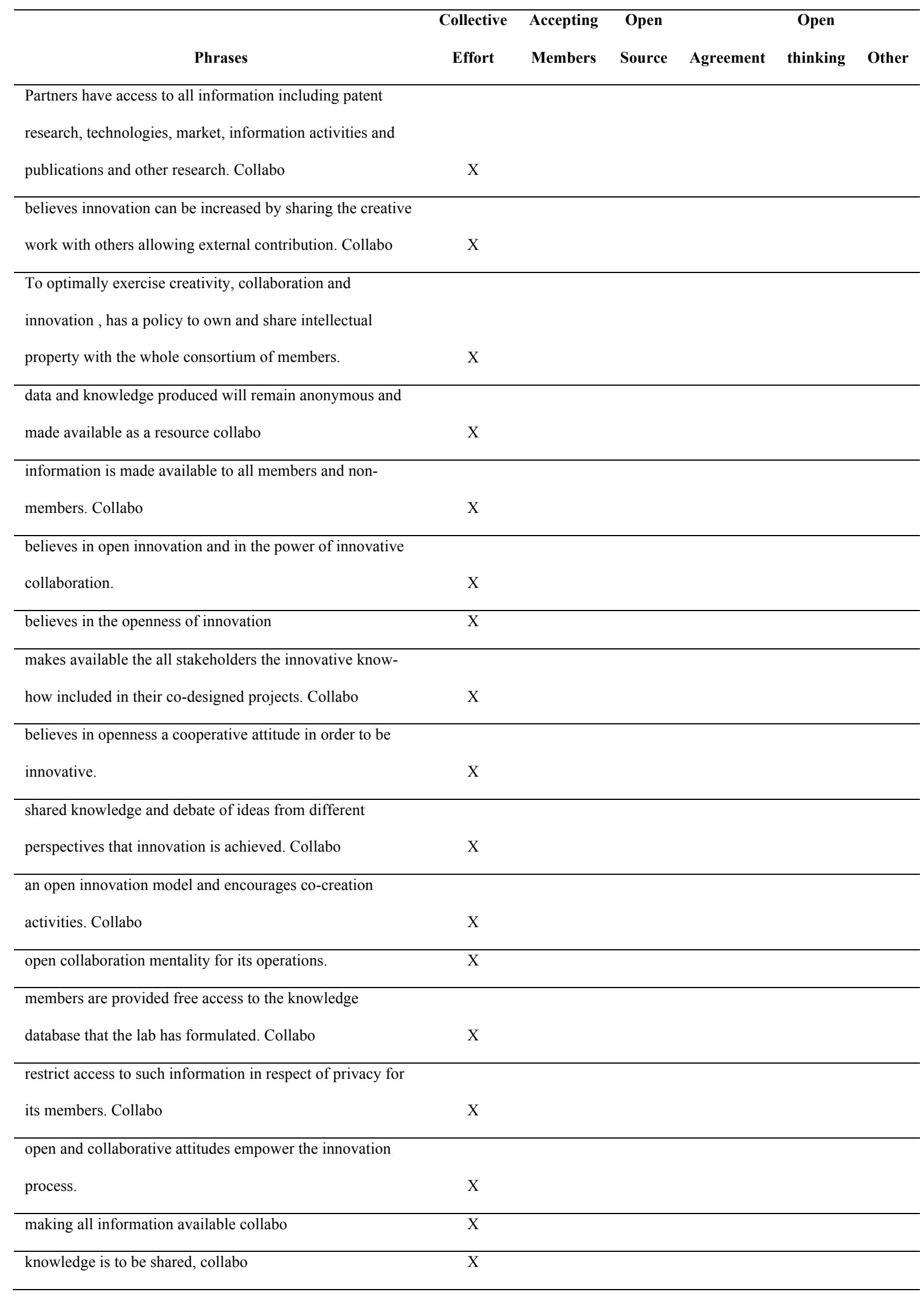




\begin{tabular}{|c|c|c|c|c|c|c|}
\hline \multirow[b]{2}{*}{ Phrases } & \multirow{2}{*}{$\begin{array}{c}\text { Collective } \\
\text { Effort }\end{array}$} & \multirow{2}{*}{$\begin{array}{l}\text { Accepting } \\
\text { Members }\end{array}$} & \multirow{2}{*}{$\begin{array}{c}\text { Open } \\
\text { Source }\end{array}$} & \multirow[b]{2}{*}{ Agreement } & \multirow{2}{*}{$\begin{array}{c}\text { Open } \\
\text { thinking }\end{array}$} & \multirow[b]{2}{*}{ Other } \\
\hline & & & & & & \\
\hline \multicolumn{7}{|l|}{ publish and release non-copyrighted and non-confidential } \\
\hline material (unless otherwise restricted by authors). Collabo & $\mathrm{X}$ & & & & & \\
\hline willing to accept to members into the ecosystem. & & $\mathrm{X}$ & & & & \\
\hline external strategy at attracting outside investors accept & & $\mathrm{X}$ & & & & \\
\hline \multicolumn{7}{|l|}{ welcoming of all peoples and organizations that have } \\
\hline innovative ideas but limited resources & & $\mathrm{X}$ & & & & \\
\hline accepting new companies, associations, regional entities & & $\mathrm{X}$ & & & & \\
\hline \multicolumn{7}{|l|}{ does not discriminate against private or public organizations } \\
\hline \multicolumn{7}{|l|}{ or associations that wish to conduct user-centered research. } \\
\hline Accept & & $\mathrm{X}$ & & & & \\
\hline open to new members & & $\mathrm{X}$ & & & & \\
\hline open to members women seeking help. & & $\mathrm{X}$ & & & & \\
\hline \multicolumn{7}{|l|}{ open to accepting new partners who are interested in } \\
\hline participating given they contribute value. & & $\mathrm{X}$ & & & & \\
\hline \multicolumn{7}{|l|}{ utilizing open standards to simplify and promote the } \\
\hline integration of third party products into their offerings. & & & $\mathrm{X}$ & & & \\
\hline \multicolumn{7}{|l|}{ incorporates and offers free software so that members can } \\
\hline operate without restriction. & & & $\mathrm{X}$ & & & \\
\hline use as much open source software as possible. Use of & & & $\mathrm{X}$ & & & \\
\hline \multicolumn{7}{|l|}{ To reduce most complications regarding IP, the lab tries to } \\
\hline \multicolumn{7}{|l|}{ use as much Open Source software and hardware as possible } \\
\hline use of & & & $\mathrm{X}$ & & & \\
\hline use of and continued innovation in open source. & & & $\mathrm{X}$ & & & \\
\hline \multicolumn{7}{|l|}{ continued use and development of open source tools to } \\
\hline \multicolumn{7}{|l|}{ allow for the free exchange of products, software, and data } \\
\hline between the partners. Use of & & & $\mathrm{X}$ & & & \\
\hline use of open innovation models & & & $\mathrm{X}$ & & & \\
\hline \multicolumn{7}{|l|}{ use of open standards emphasizing its advantages in } \\
\hline integrating other innovators products. & & & $\mathrm{X}$ & & & \\
\hline agreement that all members signed. & & & & $\mathrm{X}$ & & \\
\hline \multicolumn{7}{|l|}{ protects the members with confidential and non-discloser } \\
\hline agreements. & & & & $\mathrm{X}$ & & \\
\hline
\end{tabular}




\begin{tabular}{|c|c|c|c|c|c|c|}
\hline & Collective & Accepting & Open & & Open & \\
\hline Phrases & Effort & Members & Source & Agreement & thinking & Other \\
\hline \multicolumn{7}{|l|}{ agreement also dictates how additional members are } \\
\hline allowed to join. & & & & $\mathrm{X}$ & & \\
\hline \multicolumn{7}{|l|}{ all participants sign agreement that outlines the } \\
\hline engagements of the members and defines their operations & & & & $\mathrm{X}$ & & \\
\hline \multicolumn{7}{|l|}{ recognize that traditional management techniques are } \\
\hline ineffective and require remodeling. Unlearn & & & & & $\mathrm{X}$ & \\
\hline \multicolumn{7}{|l|}{ must unlearn old paradigms of closed and internal thinking } \\
\hline and begin to adopt a more open and communal philosophy. & & & & & $\mathrm{X}$ & \\
\hline \multicolumn{7}{|l|}{ belives societies responsibility to diagnose its own } \\
\hline \multicolumn{7}{|l|}{ problems, vulnerabilities, and risks and generate knowledge } \\
\hline and solutions on their own behalf. & & & & & & $\mathrm{X}$ \\
\hline \multicolumn{7}{|l|}{ believes that the freedom to create and being creative is the } \\
\hline cornerstone to innovation. & & & & & & $\mathrm{X}$ \\
\hline \multicolumn{7}{|l|}{ to provide unconditioned customer satisfaction by } \\
\hline \multicolumn{7}{|l|}{ continuously improving and supplying development } \\
\hline facilities. & & & & & & $\mathrm{X}$ \\
\hline \multicolumn{7}{|l|}{ take the necessary precautions in protecting records, } \\
\hline \multicolumn{7}{|l|}{ information and results of the labs work as to not break the } \\
\hline law. & & & & & & $\mathrm{X}$ \\
\hline
\end{tabular}

Table 12 Revenue and Purpose Data points Assorted into Respective Categories

\begin{tabular}{|c|c|c|c|c|c|c|}
\hline \multirow[b]{2}{*}{ Phrase } & \multirow[b]{2}{*}{ Consulting } & \multicolumn{5}{|c|}{ Membership } \\
\hline & & Royalties & Rent & Fees & Education & Purpose \\
\hline \multicolumn{7}{|c|}{ financial sustainability is paid services: offers; } \\
\hline \multicolumn{7}{|c|}{ training, internet marketing, and promotion of country } \\
\hline \multicolumn{7}{|c|}{ level database, data collection and publication, } \\
\hline \multicolumn{7}{|c|}{ database and survey analytics, data management, } \\
\hline revision and updates. & $\mathrm{X}$ & & & & & \\
\hline \multicolumn{7}{|c|}{ paid-service model:, conduct product evaluation on } \\
\hline behalf of external entities. & $\mathrm{X}$ & & & & & \\
\hline
\end{tabular}




\begin{tabular}{|c|c|c|c|c|c|c|}
\hline \multirow[b]{2}{*}{ Phrase } & \multirow[b]{2}{*}{ Consulting } & \multicolumn{5}{|c|}{ Membership } \\
\hline & & Royalties & Rent & Fees & Education & Purpose \\
\hline \multicolumn{7}{|l|}{ Sustainable : offer project management related } \\
\hline \multicolumn{7}{|l|}{ services, use the labs resources and conduct product } \\
\hline \multicolumn{7}{|l|}{ development. prepare documentation for European } \\
\hline \multicolumn{7}{|l|}{ Union funds, conduct feasibility studies, prepare cost- } \\
\hline \multicolumn{7}{|l|}{ benefit analysis, and environment assessments for } \\
\hline \multicolumn{7}{|l|}{ sustainability projects. consultation services and } \\
\hline \multicolumn{7}{|l|}{ lobbying procedures. incubation space for SMEs. } \\
\hline \multicolumn{7}{|l|}{ service funding. } \\
\hline \multicolumn{7}{|l|}{ offers several services (such as consultation) to both } \\
\hline society and organizations the generates income & $\mathrm{X}$ & & & & & \\
\hline \multicolumn{7}{|l|}{ through custom games and their deployed web based } \\
\hline environments. Services & $\mathrm{X}$ & & & & & \\
\hline \multicolumn{7}{|l|}{ conducts consulting services, knowledge network } \\
\hline outsourcing and digital marketing & $\mathrm{X}$ & & & & & \\
\hline \multicolumn{7}{|l|}{ offering various services to companies: website } \\
\hline \multicolumn{7}{|l|}{ maintenance and management services for a fee as } \\
\hline well as receive payment for brand sponsorship. & $\mathrm{X}$ & & & & & \\
\hline \multicolumn{7}{|l|}{ sources revenue from integration and support } \\
\hline activities provided to users. Services & $\mathrm{X}$ & & & & & \\
\hline \multicolumn{7}{|l|}{ private services the lab offers: quality inspection, } \\
\hline \multicolumn{7}{|l|}{ software ratings, innovation management, training and } \\
\hline \multicolumn{7}{|l|}{ coaching activities to the industry and individual } \\
\hline person and ICT transfer. & $\mathrm{X}$ & & & & & \\
\hline \multicolumn{7}{|l|}{ revenues from private contracts: constituting services } \\
\hline \multicolumn{7}{|l|}{ of usability, accessibility, and adoption of technology } \\
\hline \multicolumn{7}{|l|}{ by users. training for health, social and/or technical } \\
\hline support for dementia and healthcare. & $\mathrm{X}$ & & & & & \\
\hline Private investment sponsors projects that test and & & & & & & \\
\hline validate products or services via users in the territo & & & & & & \\
\hline where the company expects to deploy it. & $\mathrm{X}$ & & & & & \\
\hline sustaining financial model where by they sells & & & & & & \\
\hline products and services in the fields of sustainability & $\mathrm{X}$ & & & & & \\
\hline
\end{tabular}


Membership

\begin{tabular}{|c|c|c|c|c|c|c|}
\hline \multirow[b]{2}{*}{ Phrase } & \multirow[b]{2}{*}{ Consulting } & \multicolumn{5}{|c|}{ Membership } \\
\hline & & Royalties & Rent & Fees & Education & Purpose \\
\hline \multicolumn{7}{|l|}{ and biodiversity. } \\
\hline \multicolumn{7}{|l|}{ win commercial contracts, issue invoices and } \\
\hline complete deliveries. Services & $\mathrm{X}$ & & & & & \\
\hline \multicolumn{7}{|l|}{ revenue stream through the data mining and the } \\
\hline development of business models. Services & $\mathrm{X}$ & & & & & \\
\hline revenue from innovation services it offers & $\mathrm{X}$ & & & & & \\
\hline \multicolumn{7}{|l|}{ revenue stream through consulting services in } \\
\hline \multicolumn{7}{|l|}{ technical matters and project administration, design, } \\
\hline \multicolumn{7}{|l|}{ management and performance evaluation, software } \\
\hline \multicolumn{7}{|c|}{ engineering, optimization techniques, algorithms and } \\
\hline complexity theory as well as queuing theory. & $\mathrm{X}$ & & & & & \\
\hline consultations services & $\mathrm{X}$ & & & & & \\
\hline services rendered to third parties. & $\mathrm{X}$ & & & & & \\
\hline charging success, and to business royal & & $\mathrm{X}$ & & & & \\
\hline \multicolumn{7}{|l|}{ funds generated must be based the successfulness of } \\
\hline the involved companies. Royal & & $\mathrm{X}$ & & & & \\
\hline \multicolumn{7}{|l|}{ funding through the output of the living lab as } \\
\hline commercialized products and services. & & $\mathrm{X}$ & & & & \\
\hline \multicolumn{7}{|l|}{ output innovation products of the living lab will be } \\
\hline sold & & $\mathrm{X}$ & & & & \\
\hline \multicolumn{7}{|l|}{ business model that adopts a co-financed position } \\
\hline from deploying the output products of the lab. & & $\mathrm{X}$ & & & & \\
\hline royalties generated from the innovation outputs & & $\mathrm{X}$ & & & & \\
\hline \multicolumn{7}{|l|}{ sustainable revenue model through the innovation } \\
\hline \multicolumn{7}{|l|}{ outputs: maintain a competitive price model to the } \\
\hline user base and market prices. & & $\mathrm{X}$ & & & & \\
\hline \multicolumn{7}{|l|}{ revenue stream through the sales of its developed } \\
\hline products and services. & & $\mathrm{X}$ & & & & \\
\hline \multicolumn{7}{|c|}{ financially sustainable: offers the use of its developed } \\
\hline \multicolumn{7}{|l|}{ 3D models (Virtual City), customized augmented } \\
\hline \multicolumn{7}{|l|}{ realities that various members can pay to simulate } \\
\hline their requirements in. rent & & & $\mathrm{X}$ & & & \\
\hline
\end{tabular}




\begin{tabular}{|c|c|c|c|c|c|c|}
\hline \multirow[b]{2}{*}{ Phrase } & \multirow[b]{2}{*}{ Consulting } & \multicolumn{5}{|c|}{ Membership } \\
\hline & & Royalties & Rent & Fees & Education & Purpose \\
\hline \multicolumn{7}{|l|}{ revenue from the museums exhibitions, space rental } \\
\hline and & & & $\mathrm{X}$ & & & \\
\hline rent fees & & & $\mathrm{X}$ & & & \\
\hline rent out the lab for research & & & $\mathrm{X}$ & & & \\
\hline \multicolumn{7}{|l|}{ financial support from the participants by a } \\
\hline membership-funding model & & & & $\mathrm{X}$ & & \\
\hline membership fees & & & & $\mathrm{X}$ & & \\
\hline independently sustain through courses and workshops & & & & & $\mathrm{X}$ & \\
\hline operational costs including materials and salaries & & & & & & $\mathrm{X}$ \\
\hline to also help offset the costs. & & & & & & $\mathrm{X}$ \\
\hline to help pay for operations & & & & & & $\mathrm{X}$ \\
\hline \multicolumn{7}{|l|}{ The business model for this living lab acts as a metric } \\
\hline that demonstrates the follow through of the living lab. & & & & & & $\mathrm{X}$ \\
\hline to offset the cost of operations. & & & & & & $\mathrm{X}$ \\
\hline \multicolumn{7}{|l|}{ private business model offer a cost-effective co- } \\
\hline creation process & & & & & & $\mathrm{X}$ \\
\hline \multicolumn{7}{|l|}{ to help accelerate the labs deployment of its } \\
\hline innovation platform. & & & & & & $\mathrm{X}$ \\
\hline \multicolumn{7}{|l|}{ intended to cover operational costs and pay for the } \\
\hline costs of the equipment and its setup. & & & & & & $\mathrm{X}$ \\
\hline help pay for its operational costs and activities. & & & & & & $\mathrm{X}$ \\
\hline recover operational and investment costs. & & & & & & $\mathrm{X}$ \\
\hline \multicolumn{7}{|l|}{ offset the value of the operational costs of the labs } \\
\hline activities. & & & & & & $\mathrm{X}$ \\
\hline \multicolumn{7}{|l|}{ to support project research and product } \\
\hline demonstrations. & & & & & & $\mathrm{X}$ \\
\hline \multicolumn{7}{|l|}{ used to cover market launch attempts, sponsoring } \\
\hline \multicolumn{7}{|l|}{ project proposals searches, call for ideas and } \\
\hline development of prototyping. & & & & & & $\mathrm{X}$ \\
\hline covering the production and operation costs. & & & & & & $\mathrm{X}$ \\
\hline
\end{tabular}


Table 13 Advantages Data points Assorted into Respective Categories

\begin{tabular}{|c|c|c|c|c|c|c|c|c|c|c|}
\hline \multirow[b]{2}{*}{ Phrase } & \multicolumn{2}{|l|}{ Business } & \multirow[b]{2}{*}{ Resources } & \multirow[b]{2}{*}{ Network } & \multirow[b]{2}{*}{ Validation } & \multirow[b]{2}{*}{ Marketing } & \multirow{2}{*}{$\begin{array}{l}\text { Product } \\
\text { Outcome }\end{array}$} & \multirow[b]{2}{*}{ Framework } & \multirow{2}{*}{$\begin{array}{l}\text { Social } \\
\text { Value }\end{array}$} & \multirow[b]{2}{*}{ Investmen } \\
\hline & Development & Knowledge & & & & & & & & \\
\hline \multicolumn{11}{|l|}{ Obtaining guidance and business } \\
\hline management support & $\mathrm{X}$ & & & & & & & & & \\
\hline \multicolumn{11}{|l|}{ Public relation, promotions and business } \\
\hline staff can also be assigned & $\mathrm{X}$ & & & & & & & & & \\
\hline help with business activities & $\mathrm{X}$ & & & & & & & & & \\
\hline \multicolumn{11}{|l|}{ incubation space: access to desk space } \\
\hline \multicolumn{11}{|l|}{ and advisors to support in legal, } \\
\hline financial, cultural and practical issues & $\mathrm{X}$ & & & & & & & & & \\
\hline \multicolumn{11}{|l|}{ business incubator with physical desk } \\
\hline \multicolumn{11}{|l|}{ space and complementary consulting } \\
\hline services. & $\mathrm{X}$ & & & & & & & & & \\
\hline \multicolumn{11}{|l|}{ receive professional development } \\
\hline opportunities, & $\mathrm{X}$ & & & & & & & & & \\
\hline \multicolumn{11}{|l|}{ consulting experts that manage the } \\
\hline \multicolumn{11}{|l|}{ process of defining the project, } \\
\hline \multicolumn{11}{|l|}{ conducting the experiment, and } \\
\hline \multicolumn{11}{|l|}{ industrializing it on behalf of the } \\
\hline investor. & $\mathrm{X}$ & & & & & & & & & \\
\hline
\end{tabular}




\begin{tabular}{|c|c|c|c|c|c|c|c|c|c|c|}
\hline \multirow[b]{2}{*}{ Phrase } & \multirow{2}{*}{$\begin{array}{c}\text { Business } \\
\text { Development }\end{array}$} & \multirow[b]{2}{*}{ Knowledge } & \multirow[b]{2}{*}{ Resources } & \multirow[b]{2}{*}{ Network } & \multirow[b]{2}{*}{ Validation } & \multirow[b]{2}{*}{ Marketing } & \multirow{2}{*}{$\begin{array}{l}\text { Product } \\
\text { Outcome }\end{array}$} & \multirow[b]{2}{*}{ Framework } & \multirow{2}{*}{$\begin{array}{l}\text { Social } \\
\text { Value }\end{array}$} & \multirow[b]{2}{*}{ Investment } \\
\hline & & & & & & & & & & \\
\hline \multicolumn{11}{|l|}{ services that help with the development } \\
\hline \multicolumn{11}{|l|}{ and deployment of their innovative } \\
\hline products and services. & $\mathrm{X}$ & & & & & & & & & \\
\hline consultation expertise & $\mathrm{X}$ & & & & & & & & & \\
\hline design, configuration & $\mathrm{X}$ & & & & & & & & & \\
\hline ontology expansion & $\mathrm{X}$ & & & & & & & & & \\
\hline prototyping & $\mathrm{X}$ & & & & & & & & & \\
\hline community management & $\mathrm{X}$ & & & & & & & & & \\
\hline digital marketing. & $\mathrm{X}$ & & & & & & & & & \\
\hline \multicolumn{11}{|l|}{ professional support and help from } \\
\hline expert consultants. & $\mathrm{X}$ & & & & & & & & & \\
\hline services in knowledge management, & $\mathrm{X}$ & & & & & & & & & \\
\hline technology transfer operations & $\mathrm{X}$ & & & & & & & & & \\
\hline coaching. & $\mathrm{X}$ & & & & & & & & & \\
\hline \multicolumn{11}{|l|}{ access to support in marketing and } \\
\hline communication. & $\mathrm{X}$ & & & & & & & & & \\
\hline access to experts. & $\mathrm{X}$ & & & & & & & & & \\
\hline financial engineering & $\mathrm{X}$ & & & & & & & & & \\
\hline access to expert personnel, & $\mathrm{X}$ & & & & & & & & & \\
\hline expertise & $\mathrm{X}$ & & & & & & & & & \\
\hline
\end{tabular}




\begin{tabular}{|c|c|c|c|c|c|c|c|c|c|c|}
\hline \multirow[b]{2}{*}{ Phrase } & \multirow{2}{*}{$\begin{array}{c}\text { Business } \\
\text { Development }\end{array}$} & \multirow[b]{2}{*}{ Knowledge } & \multirow[b]{2}{*}{ Resources } & \multirow[b]{2}{*}{ Network } & \multirow[b]{2}{*}{ Validation } & \multirow[b]{2}{*}{ Marketing } & \multirow{2}{*}{$\begin{array}{l}\text { Product } \\
\text { Outcome }\end{array}$} & \multirow[b]{2}{*}{ Framework } & \multirow{2}{*}{$\begin{array}{l}\text { Social } \\
\text { Value }\end{array}$} & \multirow[b]{2}{*}{ Investment } \\
\hline & & & & & & & & & & \\
\hline professional services. & $\mathrm{X}$ & & & & & & & & & \\
\hline \multicolumn{11}{|l|}{ support from the expertise provided in } \\
\hline \multicolumn{11}{|l|}{ business, marketing, communication and } \\
\hline IT specialists. & $\mathrm{X}$ & & & & & & & & & \\
\hline project development & $\mathrm{X}$ & & & & & & & & & \\
\hline share their experiences and expertise. & & $\mathrm{X}$ & & & & & & & & \\
\hline \multicolumn{11}{|l|}{ available as well as the studies, research, } \\
\hline and & & $\mathrm{X}$ & & & & & & & & \\
\hline training that the lab conducts. & & $\mathrm{X}$ & & & & & & & & \\
\hline \multicolumn{11}{|l|}{ innovative collaboration can be } \\
\hline \multicolumn{11}{|l|}{ conducted under an operational } \\
\hline framework. & & $\mathrm{X}$ & & & & & & & & \\
\hline \multicolumn{11}{|l|}{ opportunity to work with academia and } \\
\hline government & & $\mathrm{X}$ & & & & & & & & \\
\hline \multicolumn{11}{|l|}{ cross-pollination of ideas to help each } \\
\hline \multicolumn{11}{|l|}{ actor in the generation of innovative } \\
\hline products & & $\mathrm{X}$ & & & & & & & & \\
\hline new ideas & & $\mathrm{X}$ & & & & & & & & \\
\hline support from the lab to identify problems & & $\mathrm{X}$ & & & & & & & & \\
\hline grants previous knowledge & & $\mathrm{X}$ & & & & & & & & \\
\hline
\end{tabular}




\begin{tabular}{|c|c|c|c|c|c|c|c|c|c|c|}
\hline & Business & & & & & & Product & & Social & \\
\hline Phrase & Development & Knowledge & Resources & Network & Validation & Marketing & Outcome & Framework & Value & Investment \\
\hline workshops, seminars, webinars and & & & & & & & & & & \\
\hline professional development activities to & & & & & & & & & & \\
\hline educate on upcoming technology and it & & & & & & & & & & \\
\hline usage. & & $\mathrm{X}$ & & & & & & & & \\
\hline knowledge and moral support & & $\mathrm{X}$ & & & & & & & & \\
\hline education & & $\mathrm{X}$ & & & & & & & & \\
\hline stimulate innovation & & $\mathrm{X}$ & & & & & & & & \\
\hline understand the market & & $\mathrm{X}$ & & & & & & & & \\
\hline receive experience on new and & & & & & & & & & & \\
\hline upcoming technologies & & $\mathrm{X}$ & & & & & & & & \\
\hline cross-fertilize company projects for & & & & & & & & & & \\
\hline innovative outcomes. & & $\mathrm{X}$ & & & & & & & & \\
\hline improving the quality of information an & & & & & & & & & & \\
\hline knowledge exchange & & $\mathrm{X}$ & & & & & & & & \\
\hline cross-formalization of ideas, & & $\mathrm{X}$ & & & & & & & & \\
\hline information exchange & & $\mathrm{X}$ & & & & & & & & \\
\hline provides up-to-date knowledge for use. & & $\mathrm{X}$ & & & & & & & & \\
\hline education to the members; & & $\mathrm{X}$ & & & & & & & & \\
\hline helps with information literacy & & $\mathrm{X}$ & & & & & & & & \\
\hline access to the knowledge and data that & & $\mathrm{X}$ & & & & & & & & \\
\hline
\end{tabular}




\begin{tabular}{|c|c|c|c|c|c|c|c|c|c|c|}
\hline \multirow{2}{*}{ Phrase } & \multirow{2}{*}{$\begin{array}{c}\text { Business } \\
\text { Development }\end{array}$} & \multirow[b]{2}{*}{ Knowledge } & \multirow[b]{2}{*}{ Resources } & \multirow[b]{2}{*}{ Network } & \multirow[b]{2}{*}{ Validation } & \multirow[b]{2}{*}{ Marketing } & \multirow{2}{*}{$\begin{array}{l}\text { Product } \\
\text { Outcome }\end{array}$} & \multirow[b]{2}{*}{ Framework } & \multirow{2}{*}{$\begin{array}{l}\text { Social } \\
\text { Value }\end{array}$} & \multirow[b]{2}{*}{ Investment } \\
\hline & & & & & & & & & & \\
\hline \multicolumn{11}{|l|}{ TNS LL develops during the research } \\
\hline \multicolumn{11}{|l|}{ projects they conduct. } \\
\hline \multicolumn{11}{|l|}{ transfer of information from the lab to } \\
\hline community sites. & & $\mathrm{X}$ & & & & & & & & \\
\hline technology platforms are made available & & & $\mathrm{X}$ & & & & & & & \\
\hline \multicolumn{11}{|l|}{ Use of state-of-the-art technology that } \\
\hline has yet to be commercialized. & & & $\mathrm{X}$ & & & & & & & \\
\hline \multicolumn{11}{|l|}{ users granted the chance to experience } \\
\hline trail living in the apartment & & & $\mathrm{X}$ & & & & & & & \\
\hline use of living lab facilities & & & $\mathrm{X}$ & & & & & & & \\
\hline \multicolumn{11}{|l|}{ and products that help improve their } \\
\hline conditions of their lives & & & $\mathrm{X}$ & & & & & & & \\
\hline access research facilities & & & $\mathrm{X}$ & & & & & & & \\
\hline access to network technology. & & & $\mathrm{X}$ & & & & & & & \\
\hline \multicolumn{11}{|l|}{ access to the advanced tools that connect } \\
\hline \multicolumn{11}{|l|}{ several organizations together enabling } \\
\hline communication. & & & $\mathrm{X}$ & & & & & & & \\
\hline resources from educational institutions. & & & $\mathrm{X}$ & & & & & & & \\
\hline use the labs resources, test environments & & & $\mathrm{X}$ & & & & & & & \\
\hline knowledge sharing facilities & & & $\mathrm{X}$ & & & & & & & \\
\hline
\end{tabular}




\begin{tabular}{|c|c|c|c|c|c|c|c|c|c|c|}
\hline & Business & & & & & & Product & & Social & \\
\hline Phrase & Development & Knowledge & Resources & Network & Validation & Marketing & Outcome & Framework & Value & Investment \\
\hline Use of large and sophisticated test bed & & & $\mathrm{X}$ & & & & & & & \\
\hline access to new technologies & & & $\mathrm{X}$ & & & & & & & \\
\hline \multicolumn{11}{|l|}{ free access to content generated by the } \\
\hline labs community & & & $\mathrm{X}$ & & & & & & & \\
\hline incubation space & & & $\mathrm{X}$ & & & & & & & \\
\hline development technologies & & & $\mathrm{X}$ & & & & & & & \\
\hline \multicolumn{11}{|l|}{ members the opportunity to network } \\
\hline with each other, & & & & $\mathrm{X}$ & & & & & & \\
\hline \multicolumn{11}{|l|}{ help advance collaborations and } \\
\hline sponsorship & & & & $\mathrm{X}$ & & & & & & \\
\hline \multicolumn{11}{|l|}{ generate business by means of co- } \\
\hline creation. & & & & $\mathrm{X}$ & & & & & & \\
\hline \multicolumn{11}{|l|}{ help co-launch products for an increased } \\
\hline \multicolumn{11}{|l|}{ probability of success during } \\
\hline commercialization. & & & & $\mathrm{X}$ & & & & & & \\
\hline Access to business partners & & & & $\mathrm{X}$ & & & & & & \\
\hline commitment from the government & & & & $\mathrm{X}$ & & & & & & \\
\hline \multicolumn{11}{|l|}{ access to government entities, industry } \\
\hline partners & & & & $\mathrm{X}$ & & & & & & \\
\hline networking opportunity & & & & $\mathrm{X}$ & & & & & & \\
\hline
\end{tabular}




\begin{tabular}{|c|c|c|c|c|c|c|c|c|c|c|}
\hline \multirow[b]{2}{*}{ Phrase } & \multirow{2}{*}{$\begin{array}{c}\text { Business } \\
\text { Development }\end{array}$} & \multirow[b]{2}{*}{ Knowledge } & \multirow[b]{2}{*}{ Resources } & \multirow[b]{2}{*}{ Network } & \multirow[b]{2}{*}{ Validation } & \multirow[b]{2}{*}{ Marketing } & \multirow{2}{*}{$\begin{array}{l}\text { Product } \\
\text { Outcome }\end{array}$} & \multirow[b]{2}{*}{ Framework } & \multirow{2}{*}{$\begin{array}{l}\text { Social } \\
\text { Value }\end{array}$} & \multirow[b]{2}{*}{ Investment } \\
\hline & & & & & & & & & & \\
\hline \multicolumn{11}{|l|}{ creation of business clusters with tactical } \\
\hline and strategic alliances & & & & $\mathrm{X}$ & & & & & & \\
\hline \multicolumn{11}{|l|}{ bridge members together to collaborate } \\
\hline on projects. & & & & $\mathrm{X}$ & & & & & & \\
\hline business networking & & & & $\mathrm{X}$ & & & & & & \\
\hline \multicolumn{11}{|l|}{ access to local and incoming business, } \\
\hline array of other participants & & & & $\mathrm{X}$ & & & & & & \\
\hline \multicolumn{11}{|l|}{ an environment encouraged and } \\
\hline \multicolumn{11}{|l|}{ supported to evolve their knowledge and } \\
\hline \multicolumn{11}{|l|}{ expertise, expanding their professional } \\
\hline horizon & & & & $\mathrm{X}$ & & & & & & \\
\hline ecosystem & & & & $\mathrm{X}$ & & & & & & \\
\hline network with other business & & & & $\mathrm{X}$ & & & & & & \\
\hline \multicolumn{11}{|l|}{ network with national and international } \\
\hline partners. & & & & $\mathrm{X}$ & & & & & & \\
\hline \multicolumn{11}{|l|}{ to test and evaluate the technology } \\
\hline \multicolumn{11}{|l|}{ through user studies in a realistic home } \\
\hline environment. & & & & & $\mathrm{X}$ & & & & & \\
\hline testing new technologies with real users & & & & & $\mathrm{X}$ & & & & & \\
\hline \multicolumn{11}{|l|}{ development in a user-center } \\
\hline environment & & & & & $\mathrm{X}$ & & & & & \\
\hline
\end{tabular}




\begin{tabular}{|c|c|c|c|c|c|c|c|c|c|c|}
\hline & Business & & & & & & Product & & Social & \\
\hline Phrase & Development & Knowledge & Resources & Network & Validation & Marketing & Outcome & Framework & Value & Investment \\
\hline \multicolumn{11}{|l|}{ test their products with a large number of } \\
\hline \multicolumn{11}{|l|}{ people quickly and further advance their } \\
\hline products. & & & & & $\mathrm{X}$ & & & & & \\
\hline access a large market group of test users & & & & & $\mathrm{X}$ & & & & & \\
\hline \multicolumn{11}{|l|}{, run validation and complete the } \\
\hline realizations of their products. & & & & & $\mathrm{X}$ & & & & & \\
\hline help expedite the testing phases. & & & & & $\mathrm{X}$ & & & & & \\
\hline \multicolumn{11}{|l|}{ conduct proper research open channel of } \\
\hline communication end-user & & & & & $\mathrm{X}$ & & & & & \\
\hline feedback from user communities & & & & & $\mathrm{X}$ & & & & & \\
\hline test their inventions in real-life scenarios & & & & & $\mathrm{X}$ & & & & & \\
\hline validation of their product & & & & & $\mathrm{X}$ & & & & & \\
\hline \multicolumn{11}{|l|}{ develop and validate their own } \\
\hline innovative products & & & & & $\mathrm{X}$ & & & & & \\
\hline tested and validated by real consumers. & & & & & $\mathrm{X}$ & & & & & \\
\hline user need finding. & & & & & $\mathrm{X}$ & & & & & \\
\hline trend watching & & & & & $\mathrm{X}$ & & & & & \\
\hline \multicolumn{11}{|l|}{ enhance their marketing mechanisms } \\
\hline \multicolumn{11}{|l|}{ through the labs activities during the } \\
\hline integration of all the participants. & & & & & & $\mathrm{X}$ & & & & \\
\hline
\end{tabular}




\begin{tabular}{|c|c|c|c|c|c|c|c|c|c|c|}
\hline & Business & & & & & & Product & & Social & \\
\hline Phrase & Development & Knowledge & Resources & Network & Validation & Marketing & Outcome & Framework & Value & Investment \\
\hline \multicolumn{11}{|l|}{ being recognized and included in social } \\
\hline innovation. & & & & & & $\mathrm{X}$ & & & & \\
\hline \multicolumn{11}{|l|}{ establish a market base through the labs } \\
\hline events and showcases. & & & & & & $\mathrm{X}$ & & & & \\
\hline \multicolumn{11}{|l|}{ logo/brand be included in publicized } \\
\hline material and labs advertisements. & & & & & & $\mathrm{X}$ & & & & \\
\hline \multicolumn{11}{|l|}{ Access to international markets through } \\
\hline its networked collaborative partners. & & & & & & $\mathrm{X}$ & & & & \\
\hline \multicolumn{11}{|l|}{ expanding the market of a company to } \\
\hline forging shores. & & & & & & $\mathrm{X}$ & & & & \\
\hline showcase technology & & & & & & $\mathrm{X}$ & & & & \\
\hline \multicolumn{11}{|l|}{ tap into this user community to deploy } \\
\hline their complementary products. & & & & & & $\mathrm{X}$ & & & & \\
\hline establishing a market & & & & & & $\mathrm{X}$ & & & & \\
\hline legitimize members & & & & & & $\mathrm{X}$ & & & & \\
\hline \multicolumn{11}{|l|}{ access to local markets encompassing } \\
\hline local authorities and citizens. & & & & & & $\mathrm{X}$ & & & & \\
\hline \multicolumn{11}{|l|}{ get visibility for their brand and } \\
\hline recognition & & & & & & $\mathrm{X}$ & & & & \\
\hline \multicolumn{11}{|l|}{ accelerate their development and } \\
\hline improve the quality of their application. & & & & & & & $\mathrm{X}$ & & & \\
\hline
\end{tabular}




\begin{tabular}{|c|c|c|c|c|c|c|c|c|c|c|}
\hline & Business & & & & & & Product & & Social & \\
\hline Phrase & Development & Knowledge & Resources & Network & Validation & Marketing & Outcome & Framework & Value & Investment \\
\hline \multicolumn{11}{|l|}{ new and improved applications at lower } \\
\hline cost & & & & & & & $\mathrm{X}$ & & & \\
\hline access the application anytime anywhere & & & & & & & $\mathrm{X}$ & & & \\
\hline \multicolumn{11}{|l|}{ tailored innovations that address } \\
\hline particular needs and preferences & & & & & & & $\mathrm{X}$ & & & \\
\hline components, modular plug-ins & & & & & & & $\mathrm{X}$ & & & \\
\hline \multicolumn{11}{|l|}{ reducing risks in product investment and } \\
\hline market launch & & & & & & & $\mathrm{X}$ & & & \\
\hline decrease time to market & & & & & & & $\mathrm{X}$ & & & \\
\hline receiving reliable innovative products & & & & & & & $\mathrm{X}$ & & & \\
\hline \multicolumn{11}{|l|}{ content that enriches the database and } \\
\hline multimedia & & & & & & & $\mathrm{X}$ & & & \\
\hline complementary technology & & & & & & & $\mathrm{X}$ & & & \\
\hline \multicolumn{11}{|l|}{ integration of best practices in their new } \\
\hline innovative product or service & & & & & & & $\mathrm{X}$ & & & \\
\hline \multicolumn{11}{|l|}{ fast-track innovation and business } \\
\hline development & & & & & & & $\mathrm{X}$ & & & \\
\hline use a framework & & & & & & & & $\mathrm{X}$ & & \\
\hline \multicolumn{11}{|l|}{ receive methods to reduce their energy } \\
\hline consumption & & & & & & & & $\mathrm{X}$ & & \\
\hline
\end{tabular}




\begin{tabular}{|c|c|c|c|c|c|c|c|c|c|c|}
\hline \multirow[b]{2}{*}{ Phrase } & \multirow{2}{*}{$\begin{array}{c}\text { Business } \\
\text { Development }\end{array}$} & \multirow[b]{2}{*}{ Knowledge } & \multirow[b]{2}{*}{ Resources } & \multirow[b]{2}{*}{ Network } & \multirow[b]{2}{*}{ Validation } & \multirow[b]{2}{*}{ Marketing } & \multirow{2}{*}{$\begin{array}{c}\text { Product } \\
\text { Outcome }\end{array}$} & \multirow[b]{2}{*}{ Framework } & \multirow{2}{*}{$\begin{array}{l}\text { Social } \\
\text { Value }\end{array}$} & \multirow[b]{2}{*}{ Investment } \\
\hline & & & & & & & & & & \\
\hline access methods & & & & & & & & $\mathrm{X}$ & & \\
\hline research framework & & & & & & & & $\mathrm{X}$ & & \\
\hline \multicolumn{11}{|l|}{ evaluation metrics and benchmark data } \\
\hline \multicolumn{11}{|l|}{ to help improve product development } \\
\hline success. & & & & & & & & $\mathrm{X}$ & & \\
\hline \multicolumn{11}{|l|}{ learning and using the new } \\
\hline methodology/techniques in developing & & & & & & & & $\mathrm{X}$ & & \\
\hline \multicolumn{11}{|l|}{ sustainable business models for meeting } \\
\hline the needs and humans and environment. & & & & & & & & $\mathrm{X}$ & & \\
\hline method to find a niche market & & & & & & & & $\mathrm{X}$ & & \\
\hline use the labs framework & & & & & & & & $\mathrm{X}$ & & \\
\hline \multicolumn{11}{|l|}{ technology transfer plan to help move } \\
\hline \multicolumn{11}{|l|}{ ideas of one member to } \\
\hline \multicolumn{11}{|l|}{ commercialization through another } \\
\hline member & & & & & & & & $\mathrm{X}$ & & \\
\hline framework for collaborative work & & & & & & & & $\mathrm{X}$ & & \\
\hline \multicolumn{11}{|l|}{ methods and tools that help the design } \\
\hline process, testing and validation & & & & & & & & $\mathrm{X}$ & & \\
\hline sense of pride for the city & & & & & & & & & $\mathrm{X}$ & \\
\hline \multicolumn{11}{|l|}{ economic growth with the potential of } \\
\hline new job availabilities. & & & & & & & & & $\mathrm{X}$ & \\
\hline
\end{tabular}




\begin{tabular}{|c|c|c|c|c|c|c|c|c|c|}
\hline \multicolumn{2}{|l|}{ Business } & \multirow[b]{2}{*}{ Resources } & \multirow[b]{2}{*}{ Network } & \multirow[b]{2}{*}{ Validation } & \multirow[b]{2}{*}{ Marketing } & \multirow{2}{*}{$\begin{array}{l}\text { Product } \\
\text { Outcome }\end{array}$} & \multicolumn{3}{|c|}{ Social } \\
\hline Development & Knowledge & & & & & & Framework & Value & Investment \\
\hline growth of local market. & & & & & & & & $\mathrm{X}$ & \\
\hline \multicolumn{10}{|l|}{ energy management tools that will help } \\
\hline \multicolumn{10}{|l|}{ save them money and create a cleaner } \\
\hline environment. & & & & & & & & $\mathrm{X}$ & \\
\hline \multicolumn{10}{|l|}{ a mechanism to generate wealth within } \\
\hline the region. & & & & & & & & $\mathrm{X}$ & \\
\hline business ventures & & & & & & & & $\mathrm{X}$ & \\
\hline \multicolumn{10}{|l|}{ chance to be part of new social } \\
\hline \multicolumn{10}{|l|}{ enterprises, which is a whole new class } \\
\hline of business types for turkey. & & & & & & & & $\mathrm{X}$ & \\
\hline \multicolumn{10}{|l|}{ facilitate business generation and } \\
\hline growth. & & & & & & & & $\mathrm{X}$ & \\
\hline \multicolumn{10}{|l|}{ suitable environment for companies and } \\
\hline others to achieve innovation $R \& D$. & & & & & & & & $\mathrm{X}$ & \\
\hline \multicolumn{10}{|l|}{ opportunity to explore new forms of } \\
\hline innovation processes & & & & & & & & & $\mathrm{X}$ \\
\hline the added investment from local and foreign projects & & & & & & & & & $\mathrm{X}$ \\
\hline get financial aid to help drive research & & & & & & & & & $\mathrm{X}$ \\
\hline Access to funds & & & & & & & & & $\mathrm{X}$ \\
\hline finding investment & & & & & & & & & $\mathrm{X}$ \\
\hline receive scholarships for personal growth & & & & & & & & & $\mathrm{X}$ \\
\hline
\end{tabular}




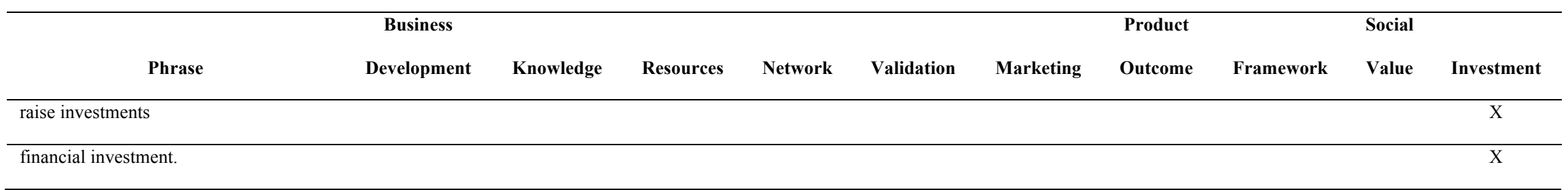


Table 14 Reasons of Communication Data points Assorted into Respective Categories

\begin{tabular}{|c|c|c|c|c|c|}
\hline Phrases & Connect/Engage & Update & Promotion & Management & Educa1 \\
\hline \multicolumn{6}{|l|}{ to have a meeting of the minds (users and } \\
\hline service providers) to discuss ideas engage & $\mathrm{X}$ & & & & \\
\hline \multicolumn{6}{|l|}{ Communication with the public and fellow } \\
\hline members engage & $x$ & & & & \\
\hline \multicolumn{6}{|l|}{ allows for collaboration for innovation by the } \\
\hline members. Engage & $x$ & & & & \\
\hline \multicolumn{6}{|l|}{ in international collaborative tasks to make a } \\
\hline presence and demonstrate engage & $\mathrm{X}$ & & & & \\
\hline \multicolumn{6}{|l|}{ to achieve two-way communication with the } \\
\hline public to & $x$ & & & & \\
\hline \multicolumn{6}{|l|}{ to interact with its users, updating on projects } \\
\hline and learning from their feedback. & $x$ & & & & \\
\hline to form a two-way communication channel. & $\mathrm{X}$ & & & & \\
\hline \multicolumn{6}{|l|}{ to maintain an open communication channel } \\
\hline with its ministries. Engage & $x$ & & & & \\
\hline to host brainstorming to generate ideas. Connect & $\mathrm{X}$ & & & & \\
\hline \multicolumn{6}{|l|}{ communication between its members and the } \\
\hline general public. Engage & $x$ & & & & \\
\hline website as the first point of contact engage & $\mathrm{X}$ & & & & \\
\hline \multicolumn{6}{|l|}{ a database and portfolio of the collaborative } \\
\hline works of the living lab. Engage & $\mathrm{X}$ & & & & \\
\hline \multicolumn{6}{|l|}{ is also utilized to more extensively connect the } \\
\hline members together. & $x$ & & & & \\
\hline \multicolumn{6}{|l|}{ to maintain the flow of information between } \\
\hline themselves and the members. Two-way & $x$ & & & & \\
\hline support of its users and investors. Engage & $\mathrm{X}$ & & & & \\
\hline \multicolumn{6}{|l|}{ to create a two-way medium that allows for } \\
\hline \multicolumn{6}{|l|}{ effective exchange of information between } \\
\hline members, the public and the lab. & $\mathrm{X}$ & & & & \\
\hline to connect with their users. & $\mathrm{X}$ & & & & \\
\hline \multicolumn{6}{|l|}{ to build up a following and partnership with } \\
\hline business. Collaboration engage & $x$ & & & & \\
\hline
\end{tabular}




\begin{tabular}{|c|c|c|c|c|c|c|}
\hline Phrases & Connect/Engage & Update & Promotion & Management & Educate & Other \\
\hline \multicolumn{7}{|l|}{ to strengthen the conduit with its user group. } \\
\hline Collaboration engage & $x$ & & & & & \\
\hline to communicate with its participants. Engage & $\mathrm{x}$ & & & & & \\
\hline \multicolumn{7}{|l|}{ multidirectional communication as way to stay } \\
\hline in touch with its members. Two-way & $x$ & & & & & \\
\hline \multicolumn{7}{|l|}{ to communicate with its members about the } \\
\hline projects. Two-way & $\mathrm{x}$ & & & & & \\
\hline to also stay connected with citizens. & $\mathrm{x}$ & & & & & \\
\hline \multicolumn{7}{|l|}{ to have an online presence and connect with its } \\
\hline members. & $\mathrm{x}$ & & & & & \\
\hline \multicolumn{7}{|l|}{ enable a true two-way communication channel } \\
\hline as well as & $\mathrm{x}$ & & & & & \\
\hline \multicolumn{7}{|l|}{ To expand their access to other forms of users, } \\
\hline collaboration engage & $\mathrm{x}$ & & & & & \\
\hline \multicolumn{7}{|l|}{ and network groups together for better } \\
\hline collaboration. Engage & $\mathrm{x}$ & & & & & \\
\hline \multicolumn{7}{|l|}{ learn perceptions of the problems that need to } \\
\hline be solved & $\mathrm{x}$ & & & & & \\
\hline update the public of its on going work. & & $\mathrm{x}$ & & & & \\
\hline \multicolumn{7}{|l|}{ disseminate and other information about the } \\
\hline labs activities. & & $x$ & & & & \\
\hline public dissemination of its works & & $\mathrm{x}$ & & & & \\
\hline \multicolumn{7}{|l|}{ disseminate updates of their activities and share } \\
\hline information with their members. & & $\mathrm{x}$ & & & & \\
\hline \multicolumn{7}{|l|}{ the community can follow the labs events, work, } \\
\hline and presentations update & & $x$ & & & & \\
\hline \multicolumn{7}{|l|}{ to expose their population to the innovative } \\
\hline products and services developed, update & & $x$ & & & & \\
\hline \multicolumn{7}{|l|}{ communicate their activities, objectives and } \\
\hline progress. & & $x$ & & & & \\
\hline \multicolumn{7}{|l|}{ to update of the progress and effectiveness of } \\
\hline the projects. & & $x$ & & & & \\
\hline to highlight stories. & & $x$ & & & & \\
\hline
\end{tabular}




\begin{tabular}{|c|c|c|c|c|c|c|}
\hline Phrases & Connect/Engage & Update & Promotion & Management & Educate & Other \\
\hline \multicolumn{7}{|l|}{ to host and dissemination vital information } \\
\hline about its activities and members. & & $x$ & & & & \\
\hline \multicolumn{7}{|l|}{ publicize information regarding $R \& D$ projects, } \\
\hline update topic details, & & $x$ & & & & \\
\hline \multicolumn{7}{|l|}{ to communicate its activities and furthers such } \\
\hline activities & & $\mathrm{x}$ & & & & \\
\hline \multicolumn{7}{|l|}{ push out information about the labs events and } \\
\hline activities. & & $\mathrm{x}$ & & & & \\
\hline \multicolumn{7}{|l|}{ All information activities, performance, } \\
\hline achievements, and member profiles is published & & $\mathrm{x}$ & & & & \\
\hline \multicolumn{7}{|l|}{ news and other forms of media content is } \\
\hline published to this site. Update & & $\mathrm{x}$ & & & & \\
\hline information dissemination. & & $\mathrm{x}$ & & & & \\
\hline \multicolumn{7}{|l|}{ to deliver informational papers and official } \\
\hline documentation. Update & & $\mathrm{x}$ & & & & \\
\hline \multicolumn{7}{|l|}{ publish news content updating members on the } \\
\hline labs activities, & & $\mathrm{x}$ & & & & \\
\hline \multicolumn{7}{|l|}{ to post updates about projects, news and } \\
\hline publications. & & $\mathrm{x}$ & & & & \\
\hline To spread the word of its activities, & & $\mathrm{x}$ & & & & \\
\hline to post news updates, and project agendas. & & $\mathrm{x}$ & & & & \\
\hline \multicolumn{7}{|l|}{ the community can follow the labs events, work, } \\
\hline and presentations update & & & $x$ & & & \\
\hline public recognition is achieved & & & $\mathrm{x}$ & & & \\
\hline to advertise their events and promote the lab. & & & $\mathrm{x}$ & & & \\
\hline \multicolumn{7}{|l|}{ targeting a large audience of citizens with } \\
\hline heterogeneous backgrounds. Promotion & & & $x$ & & & \\
\hline \multicolumn{7}{|l|}{ to legitimize their position in the professional } \\
\hline industry. Recog & & & $\mathrm{x}$ & & & \\
\hline promotion and company & & & $\mathrm{x}$ & & & \\
\hline \multicolumn{7}{|l|}{ push out information about the labs events and } \\
\hline activities. & & & $x$ & & & \\
\hline raise awareness and show case technologies and & & & $\mathrm{x}$ & & & \\
\hline
\end{tabular}




\begin{tabular}{|c|c|c|c|c|c|}
\hline Phrases & Connect/Engage Update & Promotion & Management & Educate & Other \\
\hline \multicolumn{6}{|l|}{ achievements. Recog } \\
\hline promotion its activities. & & $\mathrm{x}$ & & & \\
\hline to raise awareness of its endeavours. Promotion & & $\mathrm{x}$ & & & \\
\hline \multicolumn{6}{|l|}{ own brand and logo to enhance its public } \\
\hline \multicolumn{6}{|l|}{ visibility during its communication strategies. } \\
\hline Promotion & & $x$ & & & \\
\hline \multicolumn{6}{|l|}{ to gain access to traditional communication } \\
\hline markets. Promotion & & $x$ & & & \\
\hline public visibility critical to success recog & & $\mathrm{x}$ & & & \\
\hline \multicolumn{6}{|l|}{ to traffic information about its projects, promote } \\
\hline its events and organize courses. & & $x$ & & & \\
\hline \multicolumn{6}{|l|}{ for the purpose of educating and diffusing new } \\
\hline technologies and promotion of events. & & $x$ & & & \\
\hline primarily for the promotion of its events. & & $\mathrm{x}$ & & & \\
\hline and promote and share its events. & & $\mathrm{x}$ & & & \\
\hline promote events & & $\mathrm{x}$ & & & \\
\hline advertisements promotion & & $\mathrm{x}$ & & & \\
\hline \multicolumn{6}{|l|}{ The website is also used as a management } \\
\hline system to appraise the ideas & & & $x$ & & \\
\hline \multicolumn{6}{|l|}{ for monitoring of the members hence also acting } \\
\hline as a management tool for the TNS LL. & & & $x$ & & \\
\hline \multicolumn{6}{|l|}{ management tools to help manage photo and } \\
\hline \multicolumn{6}{|l|}{ video sharing, blogging, and publishing other } \\
\hline content to interact with the members. & & & $x$ & & \\
\hline \multicolumn{6}{|l|}{ free software tools such as source control } \\
\hline \multicolumn{6}{|l|}{ systems and material reservation, tracking } \\
\hline \multicolumn{6}{|l|}{ systems, and event calendars are used for } \\
\hline organized communication and management. & & & $x$ & & \\
\hline \multicolumn{6}{|l|}{ provide management tools to the members to } \\
\hline track their on going projects. & & & $x$ & & \\
\hline to educate and demonstrate their work. & & & & $\mathrm{x}$ & \\
\hline demonstrations of its technologies educate & & & & $\mathrm{x}$ & \\
\hline
\end{tabular}




\begin{tabular}{|c|c|c|c|c|c|}
\hline Phrases & Connect/Engage Update & Promotion & Management & Educate & Other \\
\hline showcase best practices, & & & & $x$ & \\
\hline \multicolumn{6}{|c|}{ to communicate information that is otherwise } \\
\hline difficult to post online. & & & & & $x$ \\
\hline \multicolumn{6}{|c|}{ hosts the databases of designs and designers, } \\
\hline \multicolumn{6}{|c|}{ allows for interactive communication between } \\
\hline parties. & & & & & $x$ \\
\hline
\end{tabular}

\section{Table 15 Methods Data points Assorted into Respective Categories}

\begin{tabular}{|c|c|c|c|c|c|c|}
\hline Phrases & Ethics & Testing & Commercialization & Support & Motivation & Communication \\
\hline \multicolumn{7}{|l|}{ followed ethical protocol and informed } \\
\hline \multicolumn{7}{|l|}{ the users of the undertaken projects and } \\
\hline \multicolumn{7}{|l|}{ received voluntary consent to } \\
\hline participate. & $\mathrm{X}$ & & & & & \\
\hline \multicolumn{7}{|l|}{ users are fully informed about the } \\
\hline \multicolumn{7}{|l|}{ projects and are asked to give written } \\
\hline consent of their involvement. & $\mathrm{X}$ & & & & & \\
\hline \multicolumn{7}{|l|}{ upholds all ethical requirements during } \\
\hline \multicolumn{7}{|l|}{ its activities and allows the users to } \\
\hline withdraw at any time. & $\mathrm{X}$ & & & & & \\
\hline \multicolumn{7}{|l|}{ The association informs the citizens } \\
\hline \multicolumn{7}{|l|}{ about the goals of the project, receive } \\
\hline \multicolumn{7}{|l|}{ signed consent and protect the citizen's } \\
\hline information. & $\mathrm{X}$ & & & & & \\
\hline \multicolumn{7}{|l|}{ co-design companies and public service } \\
\hline to ensure acceptable work procedures. & $\mathrm{X}$ & & & & & \\
\hline \multicolumn{7}{|l|}{ tested wishing the local area for } \\
\hline validation before commercialization. & & $\mathrm{X}$ & & & & \\
\hline
\end{tabular}




\begin{tabular}{|c|c|c|c|c|c|c|}
\hline Phrases & Ethics & Testing & Commercialization & Support & Motivation & Communication \\
\hline \multicolumn{7}{|l|}{ products to be tested and validated are } \\
\hline \multicolumn{7}{|l|}{ applied in real-life environments to } \\
\hline \multicolumn{7}{|l|}{ maintain realism and important life } \\
\hline variables. & & $\mathrm{X}$ & & & & \\
\hline \multicolumn{7}{|l|}{ The lab uses ObesiTIC as a way to } \\
\hline \multicolumn{7}{|l|}{ perform reality checks and trials on the } \\
\hline developed products. & & $\mathrm{X}$ & & & & \\
\hline \multicolumn{7}{|l|}{ Users consulted for testing purposes and } \\
\hline after the project for feedback. & & $\mathrm{X}$ & & & & \\
\hline \multicolumn{7}{|l|}{ drivers test the new technologies in } \\
\hline simulated and real-life environments. & & $\mathrm{X}$ & & & & \\
\hline users perform product validation. & & $\mathrm{X}$ & & & & \\
\hline \multicolumn{7}{|l|}{ users than iterate through the testing of } \\
\hline the product until it reaches satisfaction. & & $\mathrm{X}$ & & & & \\
\hline \multicolumn{7}{|l|}{ validates the outputs through } \\
\hline \multicolumn{7}{|l|}{ independent entities' that have no stake } \\
\hline in the operations of the lab. & & $\mathrm{X}$ & & & & \\
\hline \multicolumn{7}{|l|}{ uses a "prelaunch panel" of two } \\
\hline \multicolumn{7}{|l|}{ thousand people to validate result prior } \\
\hline \multicolumn{7}{|l|}{ to the official commercialization } \\
\hline process. & & $\mathrm{X}$ & & & & \\
\hline \multicolumn{7}{|l|}{ Citizens and the local community } \\
\hline \multicolumn{7}{|l|}{ providing feedback on usability and } \\
\hline \multicolumn{7}{|l|}{ functionality of the product commenting } \\
\hline on the process of use. & & $\mathrm{X}$ & & & & \\
\hline \multicolumn{7}{|l|}{ allows unrestricted access to } \\
\hline \multicolumn{7}{|l|}{ applications so that users may test and } \\
\hline \multicolumn{7}{|l|}{ validate the technology in the comfort } \\
\hline of their own home or on the go. & & $\mathrm{X}$ & & & & \\
\hline \multicolumn{7}{|l|}{ users to test to validate their } \\
\hline functionality. & & $\mathrm{X}$ & & & & \\
\hline fellow members, set a benchmark to & & & & & & \\
\hline which the product can be tested against & & $\mathrm{X}$ & & & & \\
\hline
\end{tabular}




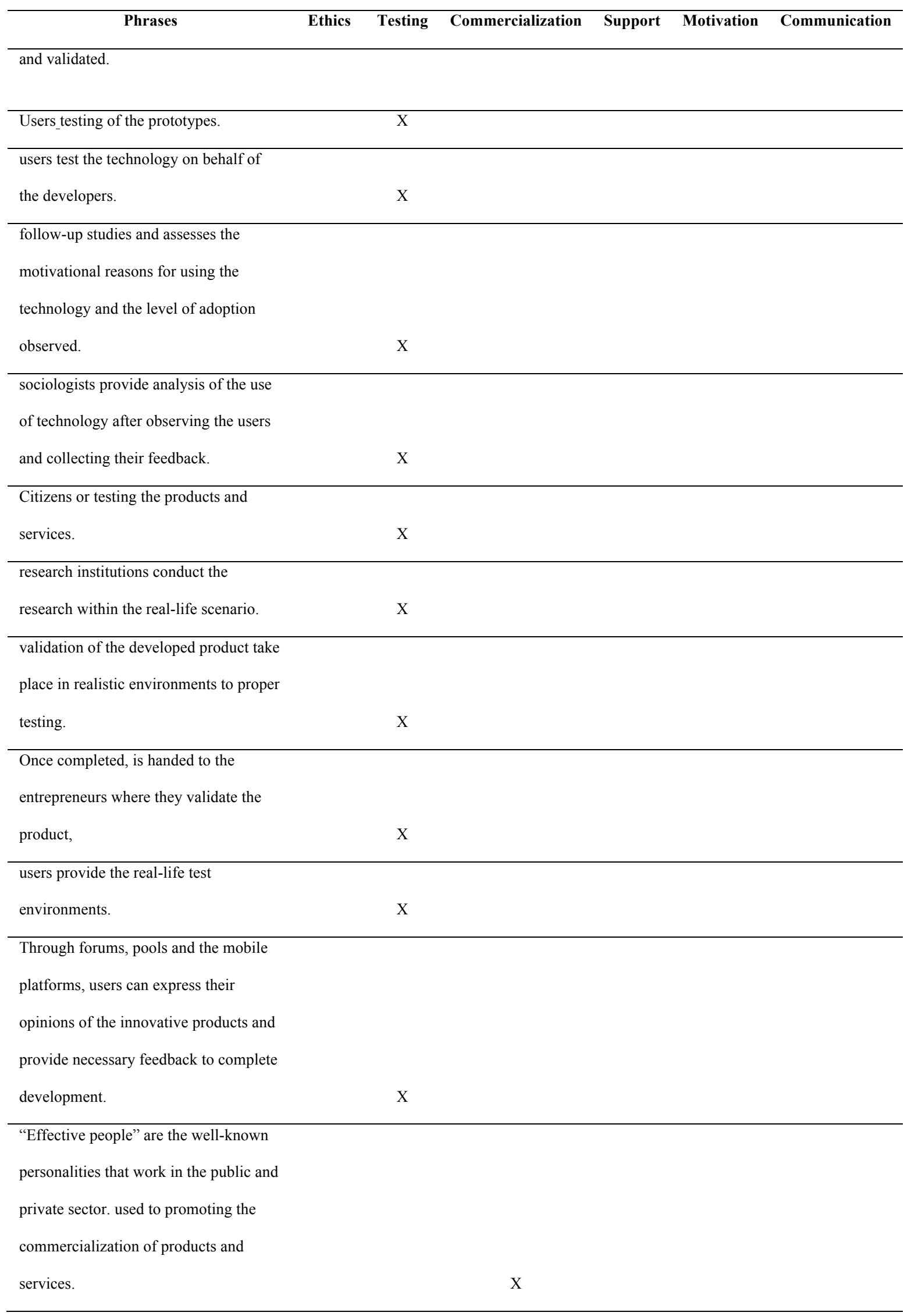




\begin{tabular}{|c|c|c|c|c|c|}
\hline Phrases & $\begin{array}{ll}\text { Ethics } & \text { Testing }\end{array}$ & Commercialization & Support & Motivation & Communication \\
\hline \multicolumn{6}{|l|}{ "Effective personalities from media" are } \\
\hline \multicolumn{6}{|l|}{ people who will help educate the public } \\
\hline \multicolumn{6}{|l|}{ about the activities of the lab and } \\
\hline \multicolumn{6}{|l|}{ encourage continued and growing } \\
\hline participation. & & $\mathrm{X}$ & & & \\
\hline \multicolumn{6}{|l|}{ marketing team then promotes and } \\
\hline \multicolumn{6}{|l|}{ pushes the product out to market. } \\
\hline \multicolumn{6}{|l|}{ nurtures the relations between actors in } \\
\hline \multicolumn{6}{|l|}{ the network to build a critical mass for } \\
\hline commercialization. & & $\mathrm{X}$ & & & \\
\hline Testers eventually becomes the & & & & & \\
\hline consumers of the new products. & & $\mathrm{X}$ & & & \\
\hline \multicolumn{6}{|l|}{ transfer the technology (i.e. file for } \\
\hline \multicolumn{6}{|l|}{ protection and licensing) and help } \\
\hline \multicolumn{6}{|l|}{ business to commercialize it through its } \\
\hline market research. & & $\mathrm{X}$ & & & \\
\hline entrepreneurs map out a business plan & & & & & \\
\hline \multicolumn{2}{|l|}{ and then commercialize the product. } & $\mathrm{X}$ & & & \\
\hline \multicolumn{6}{|l|}{ perfected, the companies can launch the } \\
\hline \multicolumn{2}{|l|}{ innovations to commercial markets. } & $\mathrm{X}$ & & & \\
\hline \multicolumn{6}{|l|}{ companies are the ones who try to } \\
\hline \multicolumn{6}{|l|}{ commercialize the innovation for the } \\
\hline \multicolumn{6}{|l|}{ purpose of dissemination and } \\
\hline \multicolumn{2}{|l|}{ exploitation. } & $\mathrm{X}$ & & & \\
\hline \multicolumn{6}{|l|}{ involves citizens to help build social } \\
\hline \multicolumn{2}{|l|}{ inclusion and social entrepreneurship. } & $\mathrm{X}$ & & & \\
\hline \multicolumn{2}{|l|}{ Organizations provid services } & & $\mathrm{X}$ & & \\
\hline \multicolumn{6}{|l|}{ provides training, workshops and e- } \\
\hline \multicolumn{2}{|l|}{ learning to empower the users. } & & $\mathrm{X}$ & & \\
\hline \multicolumn{6}{|l|}{ technical staff supporting usrs in } \\
\hline \multicolumn{6}{|l|}{ anyway in order to maintain an deep } \\
\hline \multicolumn{6}{|l|}{ understanding of the experience and } \\
\hline develop a tailored solution. & & & $\mathrm{X}$ & & \\
\hline
\end{tabular}




\begin{tabular}{|c|c|c|c|c|c|}
\hline Phrases & $\begin{array}{ll}\text { Ethics } & \text { Testing }\end{array}$ & Commercialization & Support & Motivation & Communication \\
\hline \multicolumn{6}{|l|}{ Professionals help by providing } \\
\hline evaluations and scientific knowledge & & & $\mathrm{X}$ & & \\
\hline \multicolumn{6}{|l|}{ users are educated about the technology } \\
\hline through musicals and demonstrations & & & $\mathrm{X}$ & & \\
\hline \multicolumn{6}{|l|}{ supplies its users with methods and } \\
\hline \multicolumn{6}{|l|}{ tools to enable them to produce content } \\
\hline and give feedback. & & & $\mathrm{X}$ & & \\
\hline \multicolumn{6}{|l|}{ Other tools are provided to help } \\
\hline \multicolumn{6}{|l|}{ digitally design solutions and visualized } \\
\hline on screen prior to contraction. & & & $\mathrm{X}$ & & \\
\hline \multicolumn{6}{|l|}{ uses instructional games as a teaching } \\
\hline method & & & $\mathrm{X}$ & & \\
\hline \multicolumn{6}{|l|}{ necessary environments and tools to } \\
\hline build and test the innovations. & & & $\mathrm{X}$ & & \\
\hline \multicolumn{6}{|l|}{ Businesses provide the tools and design } \\
\hline infrastructure, & & & $\mathrm{X}$ & & \\
\hline \multicolumn{6}{|l|}{ summer courses are intended to not only } \\
\hline educate the users & & & $\mathrm{X}$ & & \\
\hline \multicolumn{6}{|l|}{ leader in user communities encouraging } \\
\hline \multicolumn{6}{|l|}{ social intelligence, and influencing their } \\
\hline \multicolumn{6}{|l|}{ behaviour to adopting a participative } \\
\hline \multicolumn{6}{|l|}{ attitude towards sustainability and } \\
\hline Green IT. & & & & $\mathrm{X}$ & \\
\hline \multicolumn{6}{|l|}{ foster user communities and produce } \\
\hline \multicolumn{6}{|l|}{ editorial practices for $\mathrm{TV}$, host events } \\
\hline \multicolumn{6}{|l|}{ and also contribute to social media to } \\
\hline help and encourage user participation. & & & & $\mathrm{X}$ & \\
\hline \multicolumn{6}{|l|}{ bottom-up methodology putting users at } \\
\hline the center of the innovation process. & & & & $\mathrm{X}$ & \\
\hline \multicolumn{6}{|l|}{ mix of bottom-up and top-down } \\
\hline \multicolumn{6}{|l|}{ methodology to maximize contribution } \\
\hline \multicolumn{6}{|l|}{ from the members and not impede } \\
\hline creativity. & & & & $\mathrm{X}$ & \\
\hline
\end{tabular}




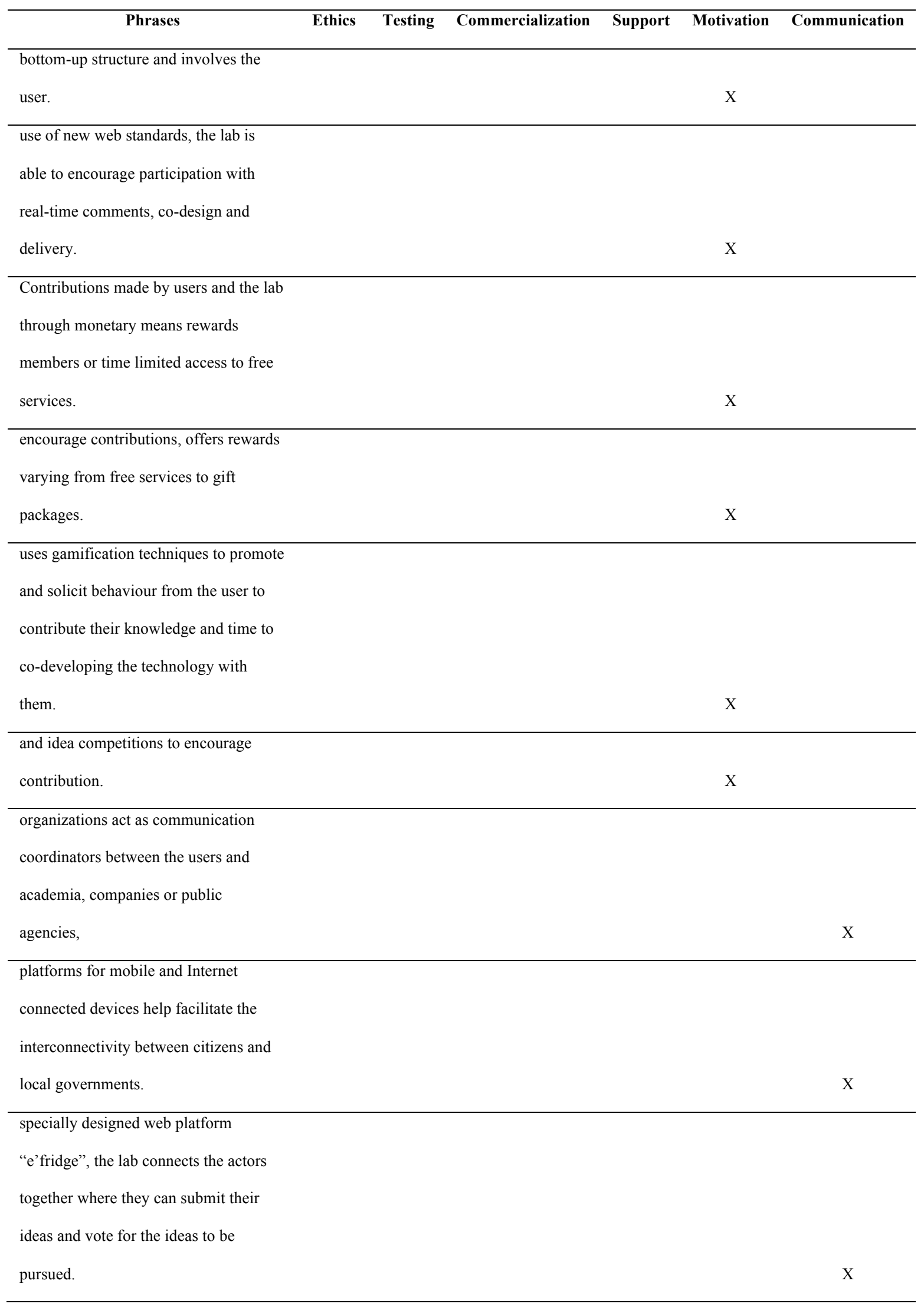




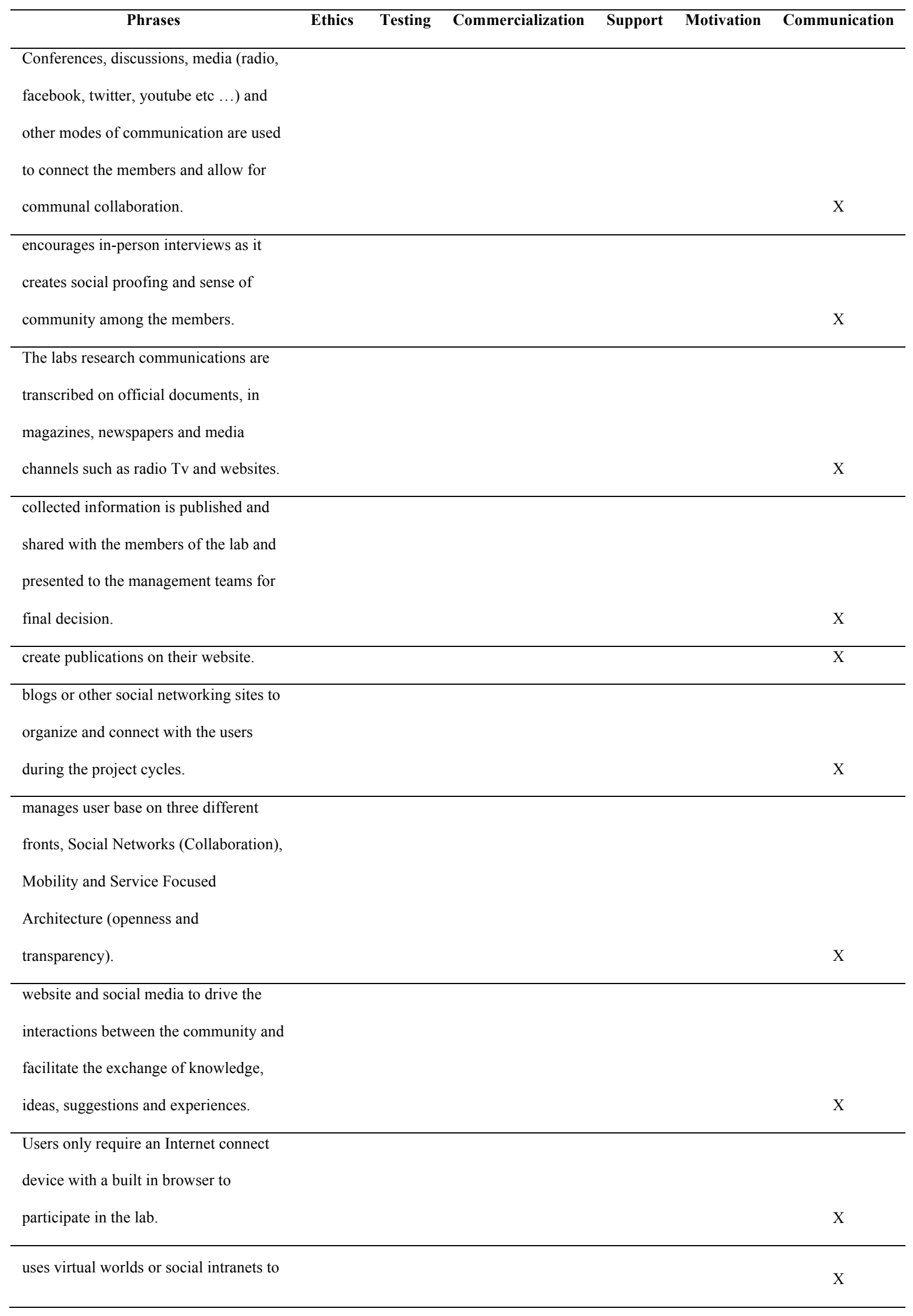




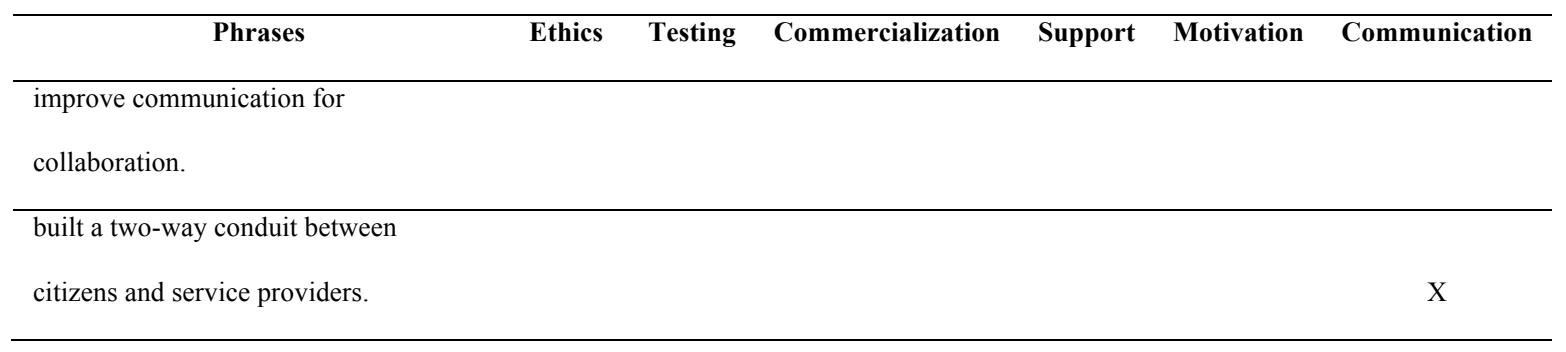

\section{Table 16 Attracting Users Data points Assorted into Respective Categories}

\begin{tabular}{|c|c|c|c|}
\hline \multirow[b]{2}{*}{ Phrases } & \multirow{2}{*}{$\begin{array}{l}\text { Members of } \\
\text { Associations }\end{array}$} & \multirow[b]{2}{*}{ Uncontrolled } & \multirow[b]{2}{*}{ Events } \\
\hline & & & \\
\hline \multicolumn{4}{|l|}{ The recruitment of citizens for testing purposes is done through civic associations in } \\
\hline order to maintain legal and ethical rules. & $\mathrm{X}$ & & \\
\hline \multicolumn{4}{|l|}{ institutes help by either attracting and organizing users as a pool of volunteers, or } \\
\hline actively recruiting users project-by-project basis. & $\mathrm{X}$ & & \\
\hline \multicolumn{4}{|l|}{ "Internet generation children and youngsters" are users who are from the very } \\
\hline successful school districts. Members & $\mathrm{X}$ & & \\
\hline aggregates, partners and committees members & $\mathrm{X}$ & & \\
\hline citizens through their association or local SME initiate some projects. & $\mathrm{X}$ & & \\
\hline \multicolumn{4}{|l|}{ users were not preselected by researchers of the lab but were by the housing authority. } \\
\hline Uncontrolled & & $\mathrm{X}$ & \\
\hline \multicolumn{4}{|l|}{ government provides access to large number of end-users and Internet hot spots for } \\
\hline access of digital functions and data on tourists, visitors, and citizens. uncontrolled & & $\mathrm{X}$ & \\
\hline from its citizens uncontrolled & & $\mathrm{X}$ & \\
\hline user comes from the publically hosted events, citizens & & & $\mathrm{X}$ \\
\hline \multicolumn{4}{|l|}{ event regular visitors and specific target groups get to participate in the co-creation } \\
\hline process of the new technologies and services. & & & $\mathrm{X}$ \\
\hline
\end{tabular}

Table 17 Data Collection Data points Assorted into Respective Categories

\begin{tabular}{|c|c|c|c|c|}
\hline Phrases & Feedbacks & Monitoring & Database & Other \\
\hline \multicolumn{5}{|c|}{ engages users by collecting their knowledge through their comments, } \\
\hline opinions and feedbacks & $\mathrm{X}$ & & & \\
\hline use focus group activities & $\mathrm{X}$ & & & \\
\hline
\end{tabular}




\begin{tabular}{|c|c|c|c|c|}
\hline Phrases & Feedbacks & Monitoring & Database & Other \\
\hline \multicolumn{5}{|l|}{ Surveys, focus groups, evaluations and meetings are held through } \\
\hline interactive websites. & $\mathrm{X}$ & & & \\
\hline \multicolumn{5}{|l|}{ uses focus groups, scenario building, story telling and surveys to extract } \\
\hline from the users the sticky information of their needs and perceptions. & $\mathrm{X}$ & & & \\
\hline \multicolumn{5}{|l|}{ hosts idea jams on the website to solicit user inputs towards problems } \\
\hline and product feedback, building up a collage of concepts and ideas. & $\mathrm{X}$ & & & \\
\hline integration of all members over Internet to share their opinions & $\mathrm{X}$ & & & \\
\hline collects data via survey & $\mathrm{X}$ & & & \\
\hline \multicolumn{5}{|l|}{ examine their feedback about the real-world activities and usability of the } \\
\hline prototypes. & $\mathrm{X}$ & & & \\
\hline to pull information (through questionnaires) about user needs feedback & $\mathrm{X}$ & & & \\
\hline \multicolumn{5}{|l|}{ "Internet generation children and youngsters" are users who are from the } \\
\hline \multicolumn{5}{|l|}{ very successful school districts. provide feedback about current lab } \\
\hline offerings. & $\mathrm{X}$ & & & \\
\hline \multicolumn{5}{|l|}{ organizes workshops (in-person and online) to showcase to the users the } \\
\hline offerings and then collect relevant feedback. & $\mathrm{X}$ & & & \\
\hline \multicolumn{5}{|l|}{ uses focus group meeting, one-on-one interviews, and surveys in addition } \\
\hline \multicolumn{5}{|l|}{ to in-field trials so that the lab can gain insight into the users thoughts } \\
\hline and opinions regarding the products and services. & $\mathrm{X}$ & & & \\
\hline \multicolumn{5}{|l|}{ using video, interviews and surveys to capture their attitudes and } \\
\hline opinions. & $\mathrm{X}$ & & & \\
\hline \multicolumn{5}{|l|}{ panel users are asked to provide their feedback using online forms or in- } \\
\hline person interviews. & $\mathrm{X}$ & & & \\
\hline \multicolumn{5}{|l|}{ channels including direct dialogue (interview), social participation } \\
\hline (workshops, forums) or feedback. & $\mathrm{X}$ & & & \\
\hline \multicolumn{5}{|l|}{ Surveys and interviews provide feedback from the users after their } \\
\hline interaction in the museum. & $\mathrm{X}$ & & & \\
\hline monitor lab & & $\mathrm{X}$ & & \\
\hline \multicolumn{5}{|l|}{ behaviours are monitored and recorded unto a back end server for future } \\
\hline analysis. & & $\mathrm{X}$ & & \\
\hline \multicolumn{5}{|l|}{ monitor Advanced webcams and media technology is used to help track } \\
\hline \multicolumn{5}{|l|}{ and data collect during the testing and implementation cycle of the } \\
\hline innovative product. & & $\mathrm{X}$ & & \\
\hline
\end{tabular}




\begin{tabular}{|c|c|c|c|c|}
\hline Phrases & Feedbacks & Monitoring & Database & Other \\
\hline \multicolumn{5}{|l|}{ does not limit itself to simple observation of users in the test } \\
\hline environment, monitor & & $\mathrm{X}$ & & \\
\hline monitor their activity via software. & & $\mathrm{X}$ & & \\
\hline \multicolumn{5}{|l|}{ advanced internet system with sensors gives the researchers the ability to } \\
\hline \multicolumn{5}{|l|}{ observe positive impact on the users life through the provided } \\
\hline technology. & & $\mathrm{X}$ & & \\
\hline \multicolumn{5}{|l|}{ uses Google analytics to observe the interactions, measuring the } \\
\hline \multicolumn{5}{|l|}{ member's demographics, visits, average time on site, new visits and } \\
\hline bounce rate. & & $\mathrm{X}$ & & \\
\hline environmental experimentation monitor & & $\mathrm{X}$ & & \\
\hline \multicolumn{5}{|l|}{ connected multiple devices over Internet to simplify the use of } \\
\hline technology and data collection. Database & & & $\mathrm{X}$ & \\
\hline conduct analysis through the database & & & $\mathrm{X}$ & \\
\hline setup databases to enable innovation development. & & & $\mathrm{X}$ & \\
\hline \multicolumn{5}{|l|}{ uses a collection of tools and methods to engage users and connect } \\
\hline \multicolumn{5}{|l|}{ functional innovation with user behaviour to better the design of the } \\
\hline products. Database & & & $\mathrm{X}$ & \\
\hline \multicolumn{5}{|l|}{ a set of platforms to manage data collection and user involvement and } \\
\hline behaviordatabase & & & $\mathrm{X}$ & \\
\hline activity public database & & & $\mathrm{X}$ & \\
\hline encourage and emphasize open dialogue between designers and users. & & & & $\mathrm{X}$ \\
\hline
\end{tabular}

Table 18 Idea generation Data points Assorted into Respective Categories

\begin{tabular}{|c|c|c|c|c|}
\hline & Set & Solution & Problem & \\
\hline Phrase & Requirements & Discovery & Discovery & Other \\
\hline \multicolumn{5}{|l|}{ Users are consulted prior to a project to gain their knowledge and input on } \\
\hline needs, & $\mathrm{X}$ & & & \\
\hline collect the needs, specifications and requirements from the ecosystem, & $\mathrm{X}$ & & & \\
\hline \multicolumn{5}{|l|}{ "Designers" contribute their experiences and knowledge to the lab expressing } \\
\hline the capabilities and limitations. Needs & $\mathrm{X}$ & & & \\
\hline \multicolumn{5}{|c|}{ All needs and requirements for innovation development comes from web based } \\
\hline forums and visitor questionnaires. & $\mathrm{X}$ & & & \\
\hline
\end{tabular}




\begin{tabular}{|c|c|c|c|c|}
\hline & Set & Solution & Problem & \\
\hline Phrase & Requirements & Discovery & Discovery & Other \\
\hline involving the handicapped individuals, able to learn about their needs & $\mathrm{X}$ & & & \\
\hline \multicolumn{5}{|l|}{ local authorities. involved during workshops expressing the needs and making } \\
\hline executive decisions on what projects to undertake. & $\mathrm{X}$ & & & \\
\hline users identify the needs & $\mathrm{X}$ & & & \\
\hline Citizens defining the requirements of the projects needs & $\mathrm{X}$ & & & \\
\hline \multicolumn{5}{|l|}{ collaborative operation between academia, business and policy makers to help } \\
\hline set the requirements and develop the necessary technology. Needs & $\mathrm{X}$ & & & \\
\hline government councils and road managers express their needs & $\mathrm{X}$ & & & \\
\hline \multicolumn{5}{|l|}{ workshops with partners and volunteers to help identify the needs and } \\
\hline requirements & $\mathrm{X}$ & & & \\
\hline users to set the out the project requirements and specification. & $\mathrm{X}$ & & & \\
\hline \multicolumn{5}{|l|}{ Users articulate their needs and define the specifications for the technologies to } \\
\hline be built. & $\mathrm{X}$ & & & \\
\hline present their technological ideas to convey the possible solutions to the users. & & $\mathrm{X}$ & & \\
\hline \multicolumn{5}{|l|}{ "Internet generation children and youngsters" are users who are from the very } \\
\hline successful school districts. help stimulate innovative ideas solution & & $\mathrm{X}$ & & \\
\hline \multicolumn{5}{|l|}{ users and organizes seminars and or workshops to brainstorm ideas for both } \\
\hline problems and solutions, & & $\mathrm{X}$ & $\mathrm{X}$ & \\
\hline \multicolumn{5}{|l|}{ uses focus groups with experts and professionals to learn about technology } \\
\hline trends and market demands. Solution problem & & $\mathrm{X}$ & $\mathrm{X}$ & \\
\hline \multicolumn{5}{|l|}{ workshops are organized with artists, young people, civil society } \\
\hline \multicolumn{5}{|l|}{ representatives and others to brainstorm and discuss problems and their } \\
\hline solutions & & $\mathrm{X}$ & $\mathrm{X}$ & \\
\hline \multicolumn{5}{|l|}{ in a workshop, the collage of members can brainstorm solutions to be } \\
\hline developed with quality feedback and commercial viability. & & $\mathrm{X}$ & & \\
\hline \multicolumn{5}{|l|}{ researchers who bring technical knowledge and enterprise members adding } \\
\hline market trend information. Problem & & & $\mathrm{X}$ & \\
\hline \multicolumn{5}{|l|}{ summer courses are intended to not only educate the users but also act as a } \\
\hline focus group where members can share ideas. & & & & $\mathrm{X}$ \\
\hline workshops to drive idea & & & & $\mathrm{X}$ \\
\hline \multicolumn{5}{|l|}{ Face-to-face or digital meeting are the primary ways of training and harnessing } \\
\hline idea collaboration. & & & & $\mathrm{X}$ \\
\hline
\end{tabular}




\begin{tabular}{|c|c|c|c|c|}
\hline 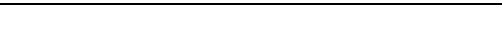 & Set & Solution & Problem & \\
\hline Phrase & Requirements & Discovery & Discovery & Other \\
\hline workshops & & & & $\mathrm{X}$ \\
\hline courses & & & & $\mathrm{X}$ \\
\hline web based forms & & & & $\mathrm{X}$ \\
\hline
\end{tabular}

\section{References}

Adler, P., Heckscher, C. \& Prusak, L. 2011. Building a collaborative enterprise. Harvard Business Review, 89(8): 94-101.

Almirall, E., \& Wareham, J. 2008. Living Labs and open innovation: roles and applicability. Electronic Journal for Virtual Organizations and Networks, 10: 21-46.

Almirall, E., Wareham, J., 2009. Innovation: a question of fit - the Living Labs approach. In: Symposium on Transversal Topics, Esade-HEC, Barcelona, Spain.

Almirall, E. \& Wareham, J. 2011. Living Labs: Arbiters of mid- and ground-level innovation. Technology Analysis \& Strategic Management, 23 (1): 87-102

Almirall, E., Lee, M. \& Wareham, J. 2012. Mapping Living Labs in the Landscape of Innovation Methodologies. Technology Innovation Management Review, September 2012: 12-18.

Asvanund, A., Clay, K., Krishnan, R., \& Smith, M. 2004. An empirical analysis of network externalities in peer-to-peer music sharing networks. Information Systems Research, 15(2): 155-174. 
Auh, S., Bell, S., McLeod, C., \& Shih, E. 2007. Co-production and customer loyalty in financial services. Journal of Retailing, 83: 359-370.

Babbie, E. 1995. The practice of social research (7 $7^{\text {th }}$ ed.). Belmont: Wadsworth Publishing Company.

Baldwin, C., Hienerth, C. and von Hippel, E. 2006. How User Innovations become Commercial Products: A Theoretical Investigation and Case Study. Research Policy, 35: 1291-313.

Ballon, P., Pierson, J., \& Delaere, S. 2005. Test and Experimentation Platforms for broadband Innovations: Examining European Practice. Conference Proceedings of 16th European Regional Conference by the International Telecommunications Society (ITS), Porto, Portugal, 4-6 September.

Barcenilla, J., Tijus, C. 2012. Ethical issues raised by the new orientations in ergonomics and living labs. Work: A Journal of Prevention, Assessment and Rehabilitation, 41: $5259-5265$

Baxter, P., \& Jack, S. 2008. Qualitative Case Study Methodology: Study Design and Implementation for Novice Researchers. The Qualitative Report, 13(4): 544-559.

Bhidé, A. 2008. The ventursome economy - how innovation sustains prosperity in a more connected world. Journal of Corporate Finance, 21(1): 8-23.

Bogers, M. \& West, J. 2012. Managing Distributed Innovation: Strategic Utilization of Open and User Innovation. Creativity \& Innovation Management, 21(1): 61-75.

Bogers, M., Afuah, A. \& Bastian, B. 2010. Users as Innovators: A Review, Critique, and 
Future Research Directions. Journal of Management, 36: 857-75.

Bonaccors, A. \& Rossi, C. 2003. Why Open Source software can succeed. Research Policy, 32(7): 1243-1258.

Boyer, K. \& Swink, M. 2008. Empirical Elephants -- Why Multiple Methods are Essential to Quality Research in Operations and Supply Chain Management. Journal of Operations Management, 26: 337-348.

Budweg, S., Schaffers, H., Ruland, R., Kristensen, K., \& Prinz, W. 2011. Enhancing Collaboration in Communities of Professionals Using a Living Lab Approach. Production Planning \& Control, 22(5/6): 594-609.

Chen, L., Marsden, J, \& Zhang, Z. 2012. Theory and Analysis of company sponsored value co-creation. Journal of Management Information systems, 29(2): 141-172.

Chesbrough, H. 2003. Open Innovation: The New Imperative for Creating And Profiting from Technology. Harvard Business School Press.

Chesbrough, H. 2011. Bringing open Innovation to services. MIT Slogan Management Review, 52(2): 85-90.

Core Labs. 2008. Building sustainable competitiveness living labs roadmap 2007-2010. Recommendations on networked systems for open user-driven research, development and innovation. Available at http://www.amicommunities.eu/pub/bscw.cgi/d310714/ Living\%20Lab\%20Roadmap\% 202007-2010.pdf 
Dahlander, L. \& Magnusson, M. (2008) Relationships between Open Source Software Companies and Communities: Observations from Nordic Firms. Research Policy, 34: $481-93$

DeFillippi, R., \& Roser, T. 2014. Aligning the co-creation project portfolio with company strategy. Strategy \& Leadership, 42(1): 30-36.

Dell'Era, C. \& Landoni, P. 2014. Living lab: A methodology between user-centred design and participatory design. Creativity and Innovation Management, 23(2): 137154.

Edwards-Schachter, M., Matti, C., \& Alcantara, E. 2013. Fostering Quality of life through Social Innovation: A Living Lab Methodology Study Case. Review of Policy Research, 29(6): 672-692.

Eiesnhardt, K. 1989. Building Theories from Case Study Research. The Academy of Management Review, 14(4): 532-550.

ENoLL, 2007. Living Labs Roadmap 2007-2010: Recommendations on Networked Systems for Open User-Driven Research, Development and Innovation. Available at http://ec.europa.eu/information_society/events/cf/document.cfm?doc_id=6474/

ENoLL. 2010. William Mitchell, father of the Living Lab concept, passed away this weekend. Available at http://www.openlivinglabs.eu/news/bill-mitchell-father-livinglab-concept-passed-away-weekend 
ENoLL. 2014. Application Form. Available at http://www.openlivinglabs.eu/livinglabs

Eriksson, M., Niitamo, V., \& Kulkki, S. (2005). State-of-the-Art in Utilizing Living Labs Approach to User-centric ICT innovation - a European approach. Luleå University of Technology, Sweden.

Evers, S., 2000. An Introduction to Open Source Software Development. Technische University, Berlin.

Fang, Y., \& Neufeld, D. 2009. Understanding sustained participation in open source software projects. Journal of Management Information Systems, 25(4): 9-50.

Folstad, A. 2008. Living labs for innovation and development of communication technology: a literature review. The Electronic Journal for Virtual Organizations and Networks, 10: 99-131.

Franke, N. \& Shah, S. 2003. How Communities Support Innovative Activities: An Exploration of Assistance and Sharing among End-Users. Research Policy, 32(1): $157-78$.

Franke, N. \& von Hippel, E. 2003. Satisfying heterogenous user needs via innovation toolkits: the case of Apache security software. Research Policy, 32(7): 1199-1215.

Fried, Y., \& Slowik, L. 2004. Enriching goal-setting theory with time: An integrated approach. Academy of Management Review, 29(3): 404-422.

Frow, P., \& Payne, A. 2011. A stakeholder perspective of the value proposition concept. 
European Journal of Marketing, 45: 223-240.

Fuller, J. \& Matzler, K. 2007. Virtual Product Experience and Customer Participation: A Chance for Customer-Centred, Really New Products. Technovation, 27: 378-87.

Fuller, J., Jawecki, G., \& Mühlbacher, H. 2007. Innovation creation by online basketball communities. Journal of Business Research, 60(1): 60-71.

Galunic, C., \& Rodan, S. 1998. Resource recombination in the firm: knowledge structures and the potential for Schumpeterian Innovation. Strategic Management Journal, 19: 1193-1201

Gassmann, O. 2006. Opening Up the Innovation Process: Towards an Agenda. $R \& \boldsymbol{D}$ Management, 36: 223-8.

Gassmann, O., Enkel, E. \& Chesbrough, H. 2010. The future of open innovation. $R \& \boldsymbol{D}$ Management, 40(3): 213-21.

Grewal, R., Lilien, G.L., \& Mallapragada, G. 2006. Location, location, location: How network embeddedness affects project success in open source systems. Management Science, 52(7): 1043-1056.

Guzman, J., Fernandez del Carpio, A., Colomo-Palacios, R., \& Velasco de Diego, M. 2013. Living Labs for User-Driven Innovation. Management Science, 56(3): 29-39.

Harwood, T., \& Garry T. 2003. An overview of content analysis. The Marketing Review, 3: $479-498$ 
Hertel, G., Niedner, S. \& Herrmann, S. 2003 Motivation of Software Developers in Open Source Projects: An Internet-Based Survey of Contributors to the Linux Kernel. Research Policy, 32(7): 1159-77.

Hienerth, C., Von Hippel, E., Jensen, A. \& Berg, M. 2012. Efficiency of Consumer (Household Sector) vs. Producer Innovation. MIT Sloan Working Paper, 4926-11.

Howe, J. 2008. Crowdsourcing: How the power of the crowd is driving the future of business. New York: Crown Publishing Group.

Hutter, K., Hautz, J., Füller, J., Mueller, J. \& Matzler, K. 2011. Communitition: The Tension between Competition and Collaboration in Community-Based Design Contests. Creativity and Innovation Management, 20: 3-21.

Jeppesen, L. \& Frederiksen, L. 2006. Why do Users Contribute to Firm-Hosted User Communities? The Case of Computer-Controlled Music Instruments. Organization Science, 17: 45-63.

Jeppesen, L. \& Lakhani, K. 2010. Marginality and Problem Solving Effectiveness in Broad- cast Search. Organization Science, 21: 1016-33.

Kannan, P., Chang, A., \& Whinston, A. 2000. Electronic communities in e-business: Their role and issues. Information Systems Frontiers, 1(4): 415-426.

Kanstrup, A., \& Christiansen, E. 2009. User-driven Points for Feedback Motivated Electricity savings in Private Households. Joint Actions on Climate Change, :1-10 
Kanstrup, A., Bjerge, K., \& Kristensen, J. 2010. A Living Laboratory Exploring Mobile Support for Everyday Life with Diabetes. Wireless Pers Commun, 53: 395-408.

Kareborn, B., Erikkson, C., Stahlbrost, A., Scensson, J. 2010. A Milieu for Innovation Defining Living Labs. Lulea Univeristy of Technology, Sweden.

Kareborn, B., Anders, B., Lassinantti, J., Davoli, L., Kuenen, S., Palmquist, L., Parns, P., Stahlborst, A., Synnes, K., Wennberg, P. 2012. User Toolkits for Citizen-centric Mobile Service Innovation. Paper presented at e-Challenges Conference Proceedings. Katzy, B. 2012. Designing Viable Business Models for Living Labs. Technology Innovation Management, September 2012: 19-24

Krippendorff, K., 2004a. Content Analysis: an Introduction to its Methodology, 2nd ed. Sage Publications, Thousand Oaks, CA.

Krippendorff, K., 2004b. Reliability in content analysis: some common misconceptions and recommendations. Human Communication Research, 30 (3): 411-433.

Kogut, B. \& Zander, U. 1992. Knowledge of the Firm, Combinative Capabilities, and the Replication of Technology. Organization Science, 3: 383- 97.

Kolbe, R., \& Burnett, M. 1991. Content-Analysis Research: An Examination of Applications with Directives for Improving Research Reliability and Objectivity. Journal of Consumer Research, 18: 243-250 
Kusiak, A., 2007. Innovation: the Living Laboratory perspective. Computer-Aided Design and Applications 4, 863-876.

Lakhani, K. \& von Hippel, E. 2003. How Open Source Software Works: 'Free' User-toUser Assistance. Research Policy, 32: 923-43.

Laursen, K. \& Salter, A. 2006. Open for Innovation: The Role of Openness in Explaining Innovation Performance among UK Manufacturing Firms. Strategic Management Journal, 27: 131-50.

Lee, S, Olson, D., \& Trim, S. 2012. Co-innovation convergences, collaboration and cocreation for organizational value. Management Decision, 50(5): 817-831.

Leminen, S. \& Westerlund, M. 2012. Towards innovation in living labs networks. International Journal of Product Development, 17(1/2): 43-59.

Leminen, S., Westerlund, M., \& Nystrom, A. 2012. Living Labs as Open-Innovation Networks. Technology Innovation Management Review, September 2012: 6-11.

Lester, S., Tomkovick, C., Wells, T., Flunker, L., \& Kickul, J. 2005. Does servicelearning add value? Examining the perspectives of multiple stakeholders. Academy of Management Learning \& Education, 4(3): 278-294.

Levén, P., Holmström, J., 2008. Consumer co-creation and the ecology of innovation: A Living Lab approach. In: Proceedings of IRIS31, August 10-13, Are, Sweden.

Liedtke, C., Welfens, M., Rohn, H. \& Nordmann, J.. 2012. LIVING LABS: user-driven innovation for sustainability. International Journal of Sustainability in Higher 
Education, 13(2): 106-118.

Mack, N., Woodsong, C., MacQueen, K.M., Guest, G. \& Namey, E. 2005. Qualitative Research Methods: A Data Collector's Field Guide. Family Health International: NC, USA.

Mattson, J. 2010. Developing a strategic abstraction tool for service innovation. Journal of Strategic Marketing, 18: 133-144.

Mele, C., Russo-Spena, T. \& Colurcio, M. 2010. Co-creating value innovation through resource integration. International Journal of Quality and Service Science, 2(1): 6078.

Miles, M. \& Huberman, A. 1994. Qualitative Data Analysis (2nd edition). Thousand Oaks, CA: Sage Publications.

Moor, K., Ketyko, I., Joseph, W., Deryckere, T., Marez, L., Martens, L., Verleye, G. 2010. Proposed framework for evaluating quality of experience in a mobile, testbedoriented living lab setting. Mobile Networks \& Applications, 15(3): 378-391.

Morrison, P., Roberts, J. \& Midgley, D. 2004. The Nature of Lead Users and Measurement of Leading Edge Status. Research Policy, 33: 351-62.

Morrison, E., \& Bies, R. 1991. Impression management in the feedback-seeking process: A literature review and research agenda. Academy of Management Review, 16(3): $522-541$.

Mulder, I. 2012. Living Labbing the Rotterdam Way: Co-Creation as an Enabler for 
Urban Innovation. Technology Innovation Management Review. September 2012: $39-43$

Niitamo, V., Westerlund, M. \& Leminen, S. 2012. A small-firm perspective on the benefits of living labs. Technology Innovation Management Review, September 2012: 44-49.

Nystrom, A.-G-, Leminen, S., Westerlund, M. \& Kortelainen, M. 2014. Actor roles and role patterns influencing innovation in living labs. Industrial Marketing Management, 43: 483-495.

O’Hern, M., \& Rindfleisch, A. 2009. Customer co-creation: A typology and research agenda. Review of Marketing Research, 6: 84-106.

Parmentier, G., \& Gandia, R. 2013. Managing Sustainable Innovation with a User Community Toolkit: the case of the video Game Trackamaion. Creativity \& Innovation Management, 22(2): 195-208.

Pierson, J., \& Lievens, B. "Configuring living labs for a 'thick' understanding of innovation," Ethnographic Praxis in Industry Conference Proceedings, 2005.

Pisano, G., \& Teece, D. 2007. How to capture value from innovation: Shaping intellectual property and industry architecture. California Management Review, 50: $278-296$.

Porter, C., \& Donthu, N. 2008. Cultivating trust and harvesting value in virtual communities. Management Science, 54: 113-128. 
Prugl, R. \& Schreier, M. 2006. Learning from Leading-Edge Customers at The Sims: Opening Up the Innovation Process using Toolkits. R\&D Management, 36: 237-50.

Ren, Y., Kraut, R., \& Kiesler, S. 2007. Applying common identity and bond theory to design of online communities. Organization Studies, 28(3): 377-408.

Russo-Spena, T., Mele, C. 2012. 'Five-Co-s' in innovating: a practice-based view. Journal of Service Management, 23(4): 527-553.

Saarijarvi, H. 2012. The mechanisms of value co-creation. Journal of strategic Marketing, 20(5): 381-391.

Sanders, E., \& Stappers P. 2008. Co-creation and the new landscapes of design. CoDesign, 4(1): 5-18.

Sawhney, M. \& Prandelli, E. 2000. Communities of Creation: Managing Distributed Innovation in Turbulent Markets. California Management Review, 42: 24-54.

Schaffers, H., Kulkki, S., 2007. Living Labs, an open innovation concept fostering rural development. Tech Monitor, Special Issue on Open Innovation: A New Paradigm in Innovation Management. September, 30-38.

Schaffers, H., \& Turkama, P. 2012. Living Labs for Cross-Border Systemic Innovation. Technology Innovation Management Review. September 2012: 25-30

Schau, H.J., Muniz, A.M. \& Arnould, E.J. 2009. How Brand Community Practices Create Value. Journal of Marketing, 73: 30-51. 
Schumacher, J., \& Niitamo, V., 2008. European Living Labs - A New Approach for Human Centric Regional Innovation. Berlin: Wissenschaftlicher Verlag Berlin.

Schuurman, D., \& De Marez, L. 2012. Structuring User Involvement in Panel-Based Living Labs. Technology Innovation Management Review. September 2012: 31-38

Sleeswijk F., Stappers P., van der Lugt R., \& Sanders E. 2005. Contextmapping: experiences from practice. CoDesign, 1(2): 119-49.

Sonpar, K. \& Golden-Biddle, K. 2008. Using Content analysis to elaborate adolescent theories of organization. Organizational Research Methods, 11(4): 795-814.

Stake, R. E. (1995). The art of case study research. Thousand Oaks, CA: Sage.

Vargo, S. \& Lusch, R.F. 2004. Evolving to a new dominant logic for marketing. Journal of Marketing, 68(1): 1-17.

Tangpong, C. 2011. Content analytic approach to measuring constructs in operations and supply chain management. Journal of Operations Management, 29: 627-638.

VINNOVA. 2009. The Innovation Platform. Available at: http://www.vinnova.se/upload/EPiStorePDF/vr-09-25.pdf

Von Hippel, E. \& Katz, R. 2002. Shifting Innovation to Users via Toolkits. Management Science, 48(7): 821-837.

Von Hippel, E. 2001. Perspective: User toolkits for Innovation. Journal of Product Innovation Management, 18: 247-57. 
Von Hippel, E. 2007. Horizontal Innovation Networks - By and For Users. Industrial and Corporate Change, 16: 293-315.

Von Hippel, E., \& von Krogh, G. 2003. Open source software and the "privatecollective" innovation model: Issues for organization science. Organization Science, 14(2): 209-223.

Von Hippel, E., \& Oliveira, P. 2011. Users as service innovators: The case of banking services. Research Policy, 40(6): 806-818.

Wasko, M., \& Faraj, S. 2005. Why should I share? Examining social capital and knowledge contribution in electronic networks of practice. MIS Quarterly, 29(1): 3557.

Wasko, M., \& Faraj, S. 2000. "It is what one does": Why people participate and help others in electronic communities of practice. Journal of Strategic Information Systems, 9(3): 155-173.

Weber, R. 1990. Basic Content Analysis, 2nd ed. Sage Publications, Newbury Park.

West, J. \& Gallagher, S. 2006. Challenges of Open Innovation: The Paradox of Firm Investment in Open-Source Software. R\&D Management, 36: 319-31.

West, J., \& O'Mahony, S. 2008. The role of participation architecture in growing sponsored open source communities. Industry and Innovation, 15(2): 145-168.

Wiertz, C., \& de Ruyter, K. 2007. Beyond the call of duty: Why customers contribute to firm- hosted commercial online communities. Organization Studies, 28(3): 347-376. 
Wolfe, R., Gephart, R., Johnson, T., 1993. Computer-facilitated qualitative data analysis: potential contributions to management research. Journal of Management, 19 (3): 637-660.

Yin, R. 2003. Case study research: Design and methods, 3rd ed.. Thousand Oaks, CA.

Zhang, X., \& Chen, R. 2008. Examining the mechanisms of the value co-creation with customers. International Journal of Production Economics, 116: 242-250.

Zhang, Z. 2010. Feeling the sense of community in social networking usage. IEEE Transactions on Engineering Management, 57(2): 225-239.

Zwass, V. 2010. Co-creation: Toward Taxonomy and an Integrated Research Perspective. International Journal of Electronic Commerce, 15: 11-48. 